\title{
The role of Holliday Junction Recognition Protein and its interactions with RNA and the Condensin II complex in human centromeric chromatin establishment
}

\author{
Meghan Cynthia Barnhart-Dailey \\ Albuquerque, NM
}

B.S. Biochemistry, University of New Mexico, 2008

M.S. Biochemistry and Molecular Genetics, University of Virginia, 2010

A Dissertation presented to the Graduate Faculty of the University of Virginia in Candidacy for the Degree of

Doctor of Philosophy

Department of Biochemistry and Molecular Genetics

University of Virginia

May, 2014 
(C) 2014 Meghan Cynthia Barnhart-Dailey 


\section{Dedication and Acknowledgements}

This thesis is dedicated to my parents. I would not be here without your guidance and support, Mom and Dad. Thank you for raising me to put God and family first in my life. Thank you for encouraging and enabling me to pursue all my dreams. Thank you for being there for every science fair, every ballet recital, and every moment in between. Thank you for all the sacrifices you made to ensure Matt, Mike, and I had all we ever needed and more. I will always be thankful that I have two such wonderful parents who I can turn to in times of joy, trial, and routine, who live their lives as examples of who I want to become someday. I am so blessed to be your daughter, I love you Mom and Dad.

I also dedicate this work to my Papa. You always told me I was just as smart as I was pretty and that I could do anything if I set my mind to it. Thank you for believing in me, for rubbing your whiskers on my cheeks, and for being the best Aggie-loving, Irish grandpa a girl could ask for. I miss you dearly, and I love you Papa. Thank you for always believing in me.

Matt and Mike, you two are my favorite people in the whole world. I am so blessed that you are my brothers. Matt, your faith, gentleness, and determination inspire me daily. You have overcome challenges far greater than anyone I know and have done it without faltering. Your strength is amazing. Thank you for being the kindest big brother there ever was, I look up to you more than you will ever know. Mike, you can make me smile faster than anyone. I swear, I can be in the worst mood and just hearing your voice makes me want to cheer up, laugh, and smile! Thank you for always teasing me and letting me be my goofy self around you. You are so smart, mature, kind, and ambitious. I 
am so very proud of you. Thank you being the best little brother there ever was. I love you both.

To my grandparents, Nana, Gaga, Grandpa Moon, and Nanny, I am blessed to have you as my examples for how to live a fruitful life. Nana, you are the matriarch of our beautiful Bailey Irish-Catholic family, and we love you so much. Thank you for being the best Nana in the world and for always being there for me. Gaga, thank you for making my childhood magical and for always believing in me. I will always treasure our times shared playing in the Del Mar sun and your magical bedtime stories. Grandpa Moon and Nanny, thank you for always loving me and supporting me. I love you Grandpa Moon, and I miss you Nanny. To my extended family, Aunt Linda, Uncle Dave, Aunt Carol, Uncle Kerwin, Aunt Laura, Uncle Kent, Uncle Joe, Aunt Sandy, Aunt Terri, Aunt Susie, Uncle Yuval, Aunt Becky, and Uncle John, thank you for all your support. I am so blessed to have such a big and loving extended family. Thank you to all my awesome cousins, we have grown up together and have had so much fun along the way. Anna Noto, you are the sister I didn't have, and I am so thankful for our friendship.

Thank you to my in-law's, the Dailey family. I feel blessed to have married into such a loving, fun, and light-hearted family. You guys really know how to live life to the fullest, and I love being a part of it. Thank you for all your love and support.

Thank you to all my friends for always being there, especially Gina, Anna, Ashley, Stephanie, Elizabeth, Chelsi, and Madison. You girls make life so much fun. I can't wait to see what adventures we will all embark on in the future, and I am so thankful that we will be there for each other along the way. 
Thank you to my advisor Dan Foltz. You taught me how to be a successful scientist. I am thankful I was able to earn my doctorate in your lab. You have been an excellent mentor, and I could not have achieved this goal without your guidance and motivation.

Last in writing but first in my heart, thank you to my husband Paul. I thank God every day for bringing us together. I could not have imagined a more perfect partner if I dreamed you into reality. You make me smile when I am grouchy, you lead me to be the best version of myself, and you make me feel so loved. It's truly a wonderful life now that you are here with me. Thank you for the countless times you drove from Lynchburg to see me during the past six years and for always arriving with plenty of smiles, love, and happiness to share. I could not have done this without your love and support each step of the way, and I am so thankful for you. You are my everything. I know God has great plans in store for us, and I am just so excited and thankful to enter each day with you by my side. Let's always keep laughing, exploring, growing, serving, praying, and loving together. Thank you for believing in me, supporting me, and being the husband I have always dreamed of, I love you so much. 


\section{Table of Contents}

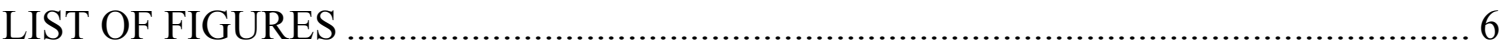

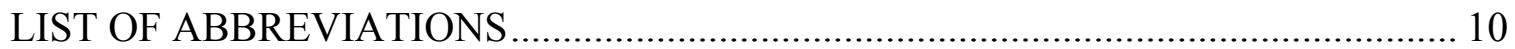

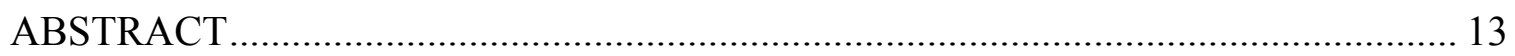

CHAPTER I: GENERAL INTRODUCTION …………........................................... 14

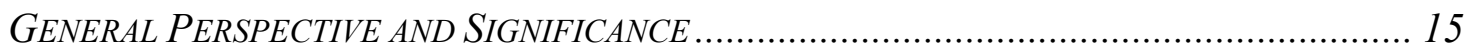

THE EPIGENETIC DEFINITION OF THE CENTROMERE …….......................................... 16

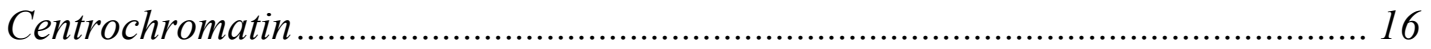

Heterochromatin Environment of Centromere Formation ………………………... 21

CENP-A AS THE CENTROMERE-SPECIFIC H3 VARIANT ……..................................... 22

THE CCAN: CONNECTING CENP-A TO THE KINETOCHORE........................................... 26

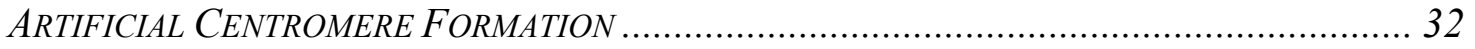

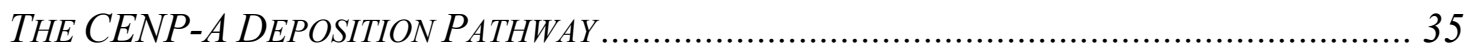

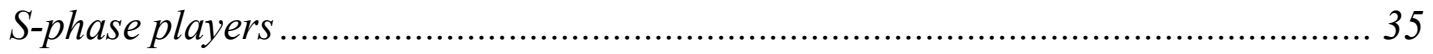

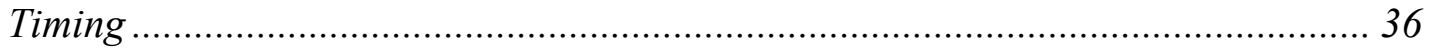

Players involved in priming: CENP-C and the Mis 18 Complex ............................ 41

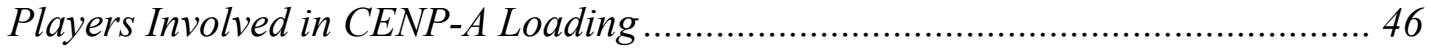

Players in CENP-A Chromatin Maturation and Stability .................................... 53

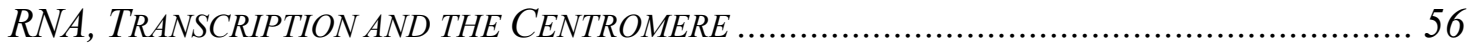

CHAPTER II: HJURP IS A CENP-A CHROMATIN ASSEMBLY FACTOR

SUFFICIENT TO FORM A FUNCTIONAL DE NOVO KINETOCHORE ................... 61 


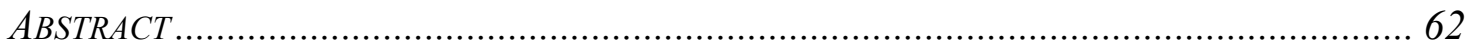

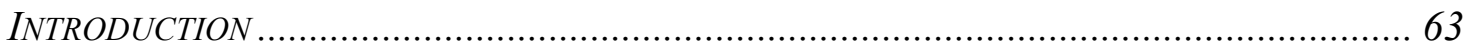

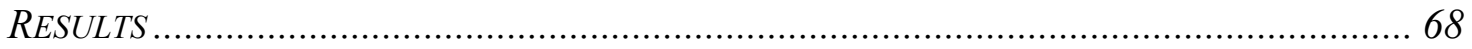

HJURP recruitment is sufficient to drive CENP-A deposition into chromatin ........ 68

CENP-A deposition by HJURP recruits CENP-A nucleosome associated proteins 74

Ectopic centromeres formed by HJURP act as kinetochores during mitosis ........... 79

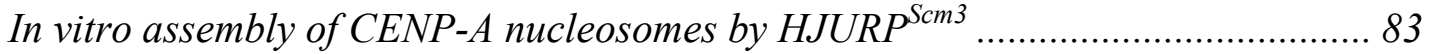

HJURP Centromeric Localization is Dependent on the Mis18 Complex at

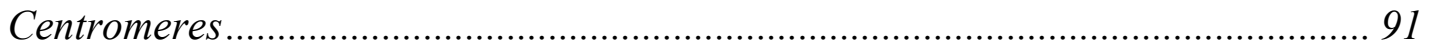

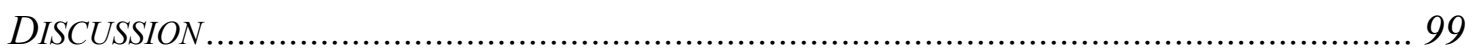

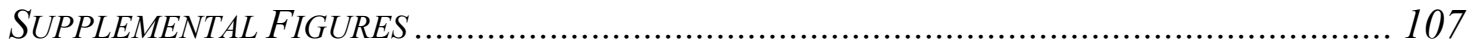

MATERIALS AND METHODS............................................................................ 123

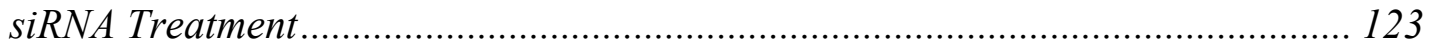

Cell culture, transfections and immunocytochemsitry.................................... 124

Mitotic Chromosome Spreads............................................................. 126

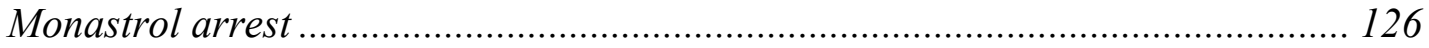

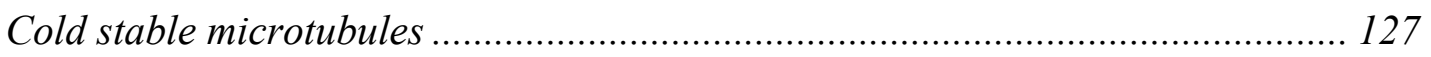

Recombinant protein purification .......................................................... 127

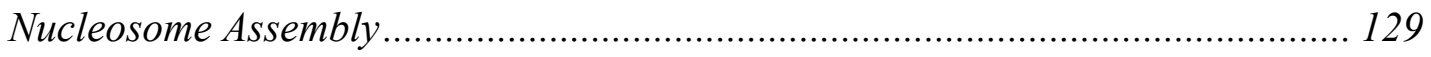

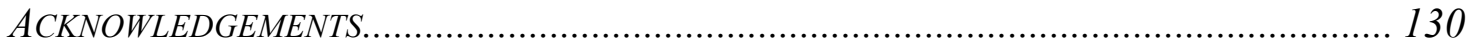

CHAPTER III: HJURP INTERACTION WITH SINE28 RNA VIA ITS VERTEBRATE

CONSERVED DOMAIN IS REQUIRED FOR CENP-A DEPOSITION .................. 131

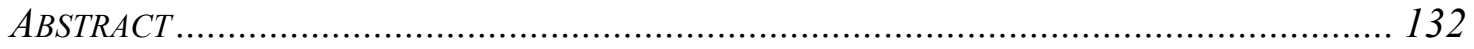


INTRODUCTION

RESULTS

HJURP vertebrate conserved domain is responsible for HJURP's nucleolar localization.

HJURP vertebrate conserved domain is required for SINE28 binding in vivo..... 148 HJURP vertebrate conserved domain is required for SINE28 binding in vitro.... 152 HJURP conserved domain is required for stable CENP-A deposition at centromeres

RNaseA + RNaseT1 cocktail treatment reduces HJURP and Mis $18 \alpha$ centromeric

recruitment 163

DISCUSSION. 170

SUPPLEMENTAL FIGURES 174

MATERIALS AND METHODS. 186

RNase treatments 186

SNAP labeling 186

Knockdown and rescue experiments....... 187

In vitro pull-down 188

Western Blotting. 188

Cell culture, transfections, and immunocytochemistry. 189 Immunoprecipitations 190

Antibodies 191

ACKNOWLEDGEMENTS. 192 


\section{CHAPTER IV: INTERACTION OF HJURP WITH THE CONDENSIN II COMPLEX}

AND ITS ROLE IN CENP-A DEPOSITION .......................................................... 193

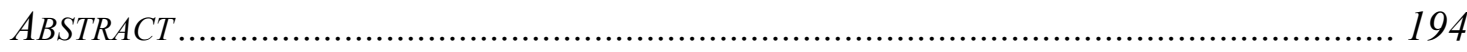

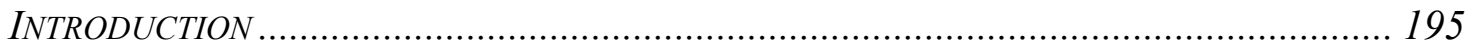

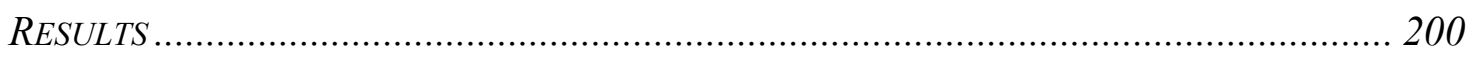

HJURP induces chromatin decondensation ................................................... 200

HJURP interacts with the Condensin II complex ............................................... 205

Depleting the Condensin II complex enhances HJURP-dependent array

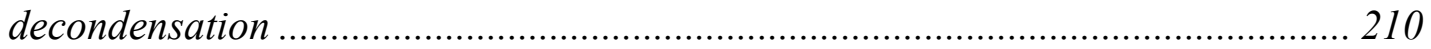

Condensin II depletion reduces HJURP centromeric recruitment ...................... 213

Condensin II depletion results in CENP-A deposition defect in human cells........ 216

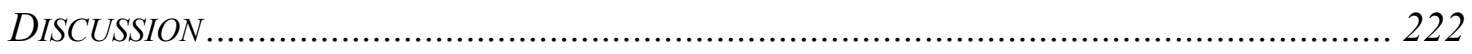

SUPPLEMENTAL FIGURES ............................................................................ 226

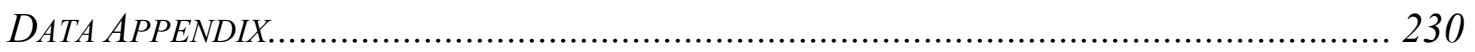

Mis 18a co-targeting can inhibit HJURP chromatin decondensation .................... 230

Mis 18 a co-targeting can bypass the requirement for condensin II recruitment .... 234

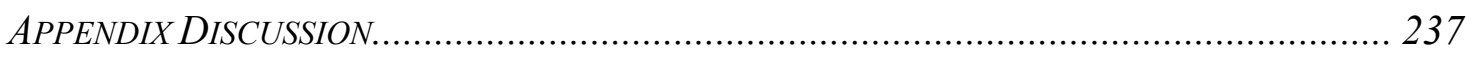

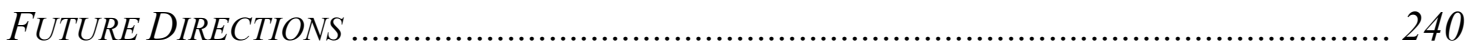

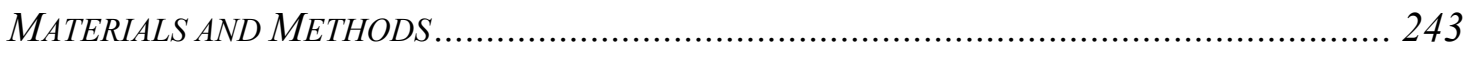

siRNA, Western Blotting, and Q-PCR ................................................... 243

Cell culture, transfections and immunocytochemistry ................................... 244

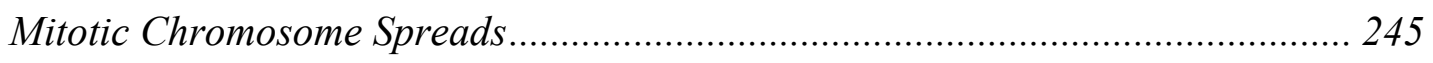

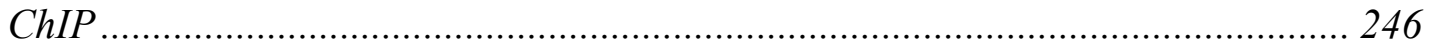


Immunoprecipitations

Inducible CENP-A cell line ................................................................................ 248

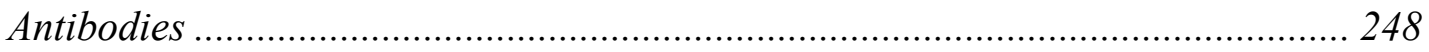

CHAPTER V: ADDITIONAL PUBLICATION CONTRIBUTIONS......................... 249

HJURP USES DISTINCT CENP-A SURFACES TO RECOGNIZE AND TO STABILIZE CENP-

A/HISTONE H4 FOR CENTROMERE ASSEMBLY ..................................................... 250

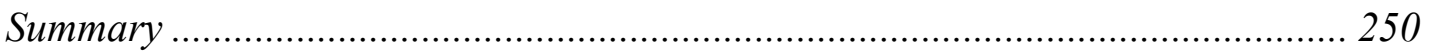

HJURP recognition of CENP-A is solely dependent on residues in the CATD...... 252

HJURP W66 is not required for stable CENP-A deposition at the LacO/TRE array

Intact CENP-A/CENP-A Interface Is Required for Nucleosome Formation.......... 257

DIMERIZATION OF THE CENP-A ASSEMBLY FACTOR HJURP IS REQUIRED FOR

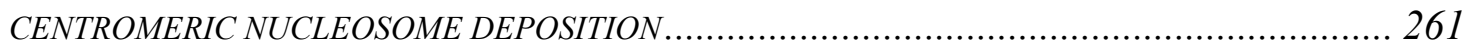

In vivo dimerization of HJURP through the carboxyl terminus ........................... 262

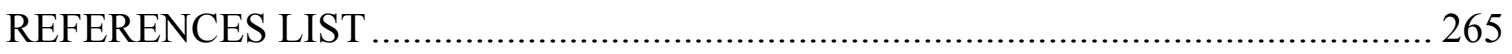

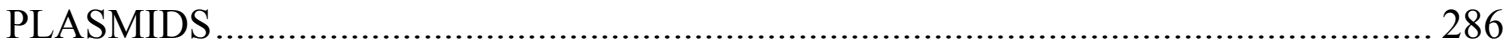

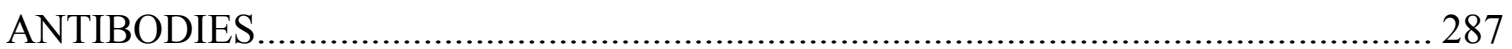

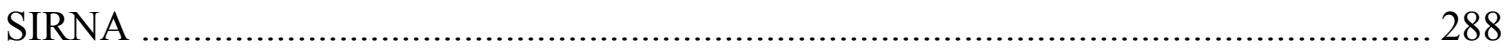




\section{List of Figures}

Figure 1-1. Schematic representation of a vertebrate monocentric centromere.............. 20

Figure 1-2. Molecular organization of the vertebrate constitutive centromere network

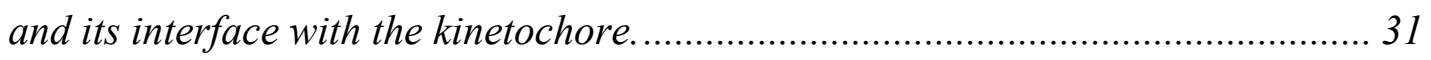

Figure 1-3: The CENP-A deposition cycle in human cells........................................ 38

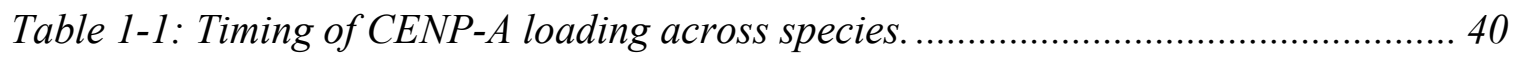

Figure 1-4: Schematic of CENP-A loading in early G1 in human cells........................ 52

Figure 1-5. Diagram of centromeric events that are influenced by centromeric

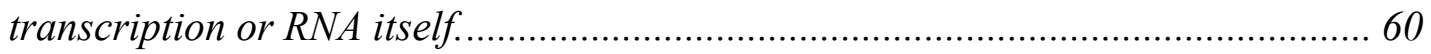

Figure 2-1. HJURP-dependent CENP-A recruitment and incorporation into the

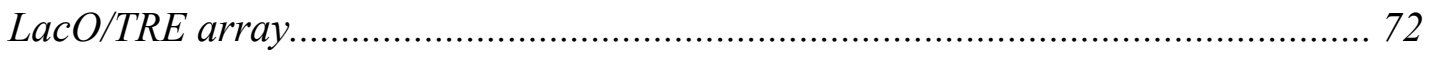

Figure 2-2. HJURP deposited CENP-A recruits constitutive centromere proteins ......... 77

Figure 2-3. Recruitment of CENP-A by HJURP ${ }^{S \mathrm{Cm} 3}$ mediates kinetochore formation at the

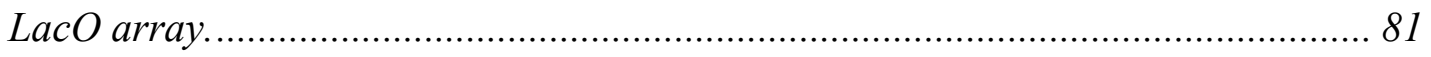

Figure 2-4. HJURP $P^{S \mathrm{~cm} 3}$ is sufficient to assemble CENP-A nucleosomes in vitro............. 87

Figure 2-5. HJURP $P^{S c m 3}$ assembled CENP-A nucleosomes are negatively supercoiled and

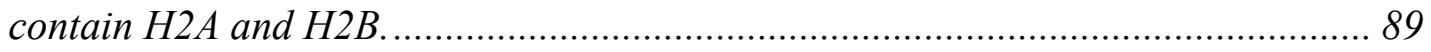

Figure 2-6. Recruitment of HJURP to Centromeres Requires the Mis18 Complex......... 93

Figure 2-7. The Mis 18 complex is not required for CENP-A deposition at the LacO/TRE

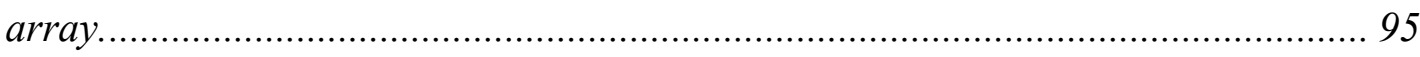

Figure 2-8. HJURP recruitment determines centromere position................................ 97 
Supplemental Figure 2-1. NIH-3T3 LacO cell line also displays stable CENP-A

deposition upon LacI-HJURP targeting.....

Supplemental Figure 2-2. CENP-A recruitment to the LacO increases over time and is stable for multiple generations after LacI-HJURP removal. 109

Supplemental Figure 2-3. Localization pattern of HJURP ${ }^{S \mathrm{~cm} 3}$. 111

Supplemental Figure 2-4. LacI-HJURP $P^{S \mathrm{~cm} 3}$ recruits CCAN components most efficiently

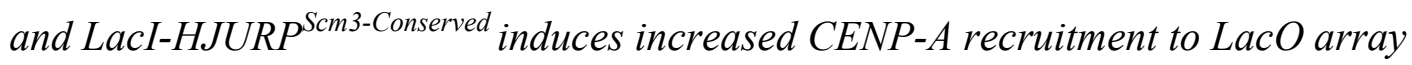
113

Supplemental Figure 2-5. Mad2 and Aurora B are recruited to LacI-HJURP $P^{\text {Scm } 3}$ assembled CENP-A arrays 115

Supplemental Figure 2-6. Recombinant purifications of MBP-HJURP $P^{S c m 3}$ and line scans of band intensities from supercoiling assay 117

Supplemental Figure 2-7. GFP-Mis18 $\alpha$ and GFP-HJURP stable lines display same localization as the endogenous proteins 119

Supplemental Figure 2-8. GFP-HJURP ${ }^{S c m} 3$-H2B causes CENP-A, CENP-C, and Hec1 mislocalization to chromosome arms. 121

Introduction Figure 3-1. SINE28 associates with HJURP and CENP-A and is present at the centromere throughout the cell cycle. 138

Introduction Figure 3-2. SINE28 is required for CENP-A deposition in human cells... 140 Figure 3-1. HJURP's vertebrate conserved domain is required for its nucleolar localization. 146

Figure 3-2. SINE28 requires the vertebrate conserved domain to interact with HJURP in vivo. 150 
Figure 3-3. HJURP binds SINE28 RNA in vitro via its vertebrate conserved domain. . 153

Figure 3-4. HJURP lacking amino acids 208-350 is unable to rescue CENP-A deposition at centromeres when endogenous HJURP is depleted. 157

Figure 3-5. $H^{4208-350}-$ GFP is not sufficient to rescue new CENP-A loading. 161

Figure 3-6. RNaseA+RNaseT1 cocktail treatment reduces $H^{1-748}-G F P$ and Mis $18 \alpha-G F P$ centromeric recruitment. 166

Figure 3-7. Model for SINE28 association with HJURP and its role in CENP-A

deposition 168

Supplemental Figure 3-1. Nucleolar localization sequence prediction. 174

Supplemental Figure 3-2. $H J^{4208-350}$ is able to efficiently bind to CENP-A. 176

Supplemental Figure 3-3. HJ $^{201-348}$ is sufficient for nucleolar localization. 178

Supplemental Figure 3-4. Npm1 association with HJURP fragments. 180

Supplemental Figure 3-5. Newly synthesized CENP-A transits the nucleolus prior to its centromere deposition. 182

Supplemental Figure 3-6. RNaseA treatment reduces $H J^{1-748}$ centromeric recruitment. 184 Figure 4-1: HJURP induces chromatin decondensation at LacO array.... 203

Figure 4-2: HJURP interacts with the Condensin II complex. 208

Figure 4-3: Depleting the Condensin II complex enhances HJURP-dependent array decondensation 211

Figure 4-4: Condensin II depletion reduces HJURP centromeric recruitment 214

Figure 4-5: Condensin II depletion results in CENP-A deposition defect in human cells 218 
Figure 4-6: Model of condensin II and HJURP at the centromere during CENP-A deposition

Supplemental Figure 4-1: Array decondensation does not reduce H3 occupancy and Npm1 does not alter array condensation state. 226

Supplemental Figure 4-2: Condensin subunits do not induce array expansion and CAPH2 can interact with HJURP during mitosis and in randomly cycling cells. 228 Appendix Figure 4-1: Mis18a co-targeting can inhibit HJURP-induced decondensation.

Appendix Figure 4-2: Mis18a inhibits Condensin II recruitment to LacI-HJ arrays .... 235 Figure 5-1. Characterization of CENP-A CATD mutant stability at the LacO/TRE array following LacI-HJURP removal 254

Figure 5-2. HJURP W66 is not required for stable CENP-A deposition at the LacO/TRE array and the CENP-A/CENP-A dimerization interface is required for stable CENPA deposition by HJURP at the LacO/TRE array 259

Figure 5-3 In vivo recruitment of HJURP through the carboxyl terminus.................... 263

Plasmid Reference Table .......................................................................................... 286

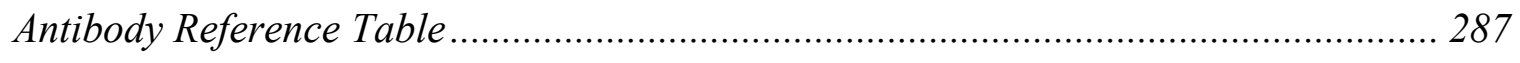

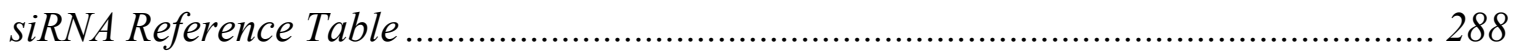




\section{List of Abbreviations}

ATP - Adenosine triphosphate

CAL1 - Chromosome alignment defect 1

CAP-H/H2/D3 -- non-SMC condensin II complex, subunit H/H2/D3

CATD - CENP-A targeting domain

CCAN - Constitutive centromere associated network

CD - Conserved domain of HJURP

Cdk1 - Cyclin dependent kinase 1

CENP-A/Cnp1/CenH3/Cse4/CID - Centromere protein A

CENP-A $^{\mathrm{CAD}}$ - CENP-A distal complex

CENP-A $^{\mathrm{NAC}}$ - CENP-A nucleosome associated complex

CENP-C-X - Centromere proteins C-X

CHD1 - Chromodomain helicase DNA binding protein 1

DAPI -- 4',6-diamidino-2-phenylindole

DDB2 -- DNA damage-binding protein 2

DNA - Deoxyribonucleic acid

dNAP1 - Nucleosome assembly protein 1

DNMT3a/3b -- DNA (cytosine-5-)-methyltransferase 3 alpha / beta

FISH - Fluorescence in situ hybridization

GAPDH -- Glyceraldehyde-3-phosphate dehydrogenase

GFP - green fluorescence protein

GST -- Glutathione $S$-transferases 
$\mathrm{H} 2 \mathrm{~A}$ - Histone H2A

H2B - Histone H2B

H3 - Histone H3

H4 - Histone H4

HA - Human influenza hemagglutinin

HCTD - HJURP C-terminal domain

HJURP/HJ - Holliday recognition protein

IgG -- Immunoglobulin G

IP -- Immunoprecipitation

IPTG -- Isopropyl $\beta$-D-1-thiogalactopyranoside

$\mathrm{kDa}$ - Kilodalton

KNL2 - Kinetochore null protein 2

LacI -- Lac repressor

LacO/TRE - Lac operator / Tet response element

LAP - Localization and affinity purification tag

LINE - Long interspersed element

LPC - Leupeptin, pepstatin, chymotrypsin

LSD1 - Lysine specific demethylase 1

MBP - Maltose binding protein

Mis18 - Missegregation of chromosomes phenotype mutant 18

Mis18BP1 - Mis18 binding protein 1

Npm1 - Nucleophosmin 1

PBS - Phosphate buffered saline 
PIPES -- 1,4-Piperazinediethanesulfonic acid

PMSF -- Phenylmethanesulfonyl fluoride

Q-PCR - Quantification polymerase chain reaction

RbAp46/48 - Retinoblastoma binding protein 46/48

RIP - RNA immunoprecipitation

RNA - Ribonucleic acid

RSF1 - Remodeling and spacing factor 1

Scm3 - Suppressor of chromosome missegregation protein 3

SINE28 - Short interspersed element 28

SMC - Structural maintenance of chromosomes

SNAP-tag -- human $\mathrm{O}^{6}$-alkylguanine-DNA alkyltransferase (hAGT)

TetR - Tet repressor

TMR-Star -- 6-carboxytetramethylrhodamine 


\begin{abstract}
$\underline{\text { Abstract }}$
The human centromere is the site on each mitotic chromosome where the kinetochore assembles. Centromeric chromatin is epigenetically defined by the presence of nucleosomes containing the histone H3 variant, CENP-A. Establishment and faithful maintenance of CENP-A chromatin at the centromere throughout the lifetime of a cell are crucial pathways for the cell to accurately maintain its ploidy. Understanding how this CENP-A chromatin is tended and what factors are required for its establishment and maintenance is the theme of this work. The first chapter of this work demonstrates the CENP-A chaperone HJURP is sufficient to deposit CENP-A nucleosomes at noncentromeric chromatin, and these nucleosomes are sufficient to nucleate de novo kinetochore formation. The second chapter characterizes an interaction between HJURP and SINE28 ssRNA, demonstrating the importance of the interaction in CENP-A deposition in human cells. The third chapter describes chromatin decondensation activity of HJURP, its interaction with the condensin II complex, and the influence of this interaction on CENP-A deposition in human cells.
\end{abstract}




\section{CHAPTER I: GENERAL INTRODUCTION}




\section{General Perspective and Significance}

Accurate chromosome segregation requires proper assembly, position, and recognition of centromeric chromatin. If centromeric chromatin is aberrant in any of these categories, chromosomal breaks and missegregations can result. These are hallmarks of genetic instability, which are often seen in cancer and birth defects. Because chromosomal instability is a hallmark and instigating event in cancer and other genetic diseases, it is important that we understand how chromosomes are faithfully inherited. Additionally, many of the proteins involved in centromere establishment, maintenance, and function are over-expressed in cancer. Elucidating how centromeres assemble is therefore an integral piece of the puzzle for identifying possible therapeutics targeting centromeric pathways. The work presented here is significant to cancer research because it increases understanding of the essential steps and proteins involved in CENP-A chromatin establishment and looks at the consequences of how they influence centromere fidelity. 


\section{The Epigenetic Definition of the Centromere}

\section{Centrochromatin}

The centromere is a single epigenetically defined region of chromatin on each chromosome that is separated from the rest of the chromatin by the presence of the centromere-specific H3 variant, CENP-A (Cse4 in S. Cerevisiae, Cnp1 in S. Pombe, CID in Drosophila). Centromeres are the foundation upon which the kinetochore is assembled during mitosis (Figure 1-1). Despite its essential role in inheritance of a cell's genetic material, many mechanistic details of how centromeric chromatin is established and maintained remain unresolved. One reason for this is that many centromere characteristics, such as underlying DNA sequence, position on the chromosome, and the players involved in establishment and maintenance, vary widely between organisms. Hallmark characteristics of this CENP-A containing "centrochromatin" are discussed here.

Perhaps the simplest centromeres are found in the budding yeast Saccharomyces cerevisiae. Centromere position in $S$. cerevisiae is determined by 125 base pairs of DNA underlying a single CENP-A/Cse4 nucleosome. The underlying AT-rich DNA of these point centromeres contains centromere DNA elements (CDEs) that recruit the sequencespecific DNA binding CBF3 complex (composed of Ndc10, Cep3, Ctf13, and Skp1), which is required to recruit the CENP-A/Cse4 deposition machinery and subsequently the kinetochore proteins.

Centromeres in the fission yeast Schizosaccharomyces pombe and higher eukaryotes like fruit flies, mice, and humans are termed regional centromeres because 
they harbor more expansive genomic regions that contain interspersed blocks of both CENP-A and H3-containing nucleosomes, but they are still monocentric in that they assemble a single centromere per chromosome. In contrast to this, the nematode $C$. elegans is holocentric because it assembles centromeres and kinetochores along the entire length of each chromosome during mitosis. The monocentric regional centromeres of fission yeast and higher eukaryotes are generally assembled on repetitive DNA sequences, though there are examples in chickens and horses where centromeres are formed on non-repetitive regions (Shang et al., 2010; Wade et al., 2009). The underlying DNA at mammalian (humans, mice) centromeres is composed of $0.5-5 \mathrm{Mb}$ of repetitive, tandem arrays of alpha-satellite DNA in humans and minor satellites in mice (Cleveland et al., 2003). D. melanogaster and S. pombe assemble their centromeres on highly repetitive DNA sequences with interspersed blocks of CENP-A and H3-containing nucleosomes, but these repeats vary from human and mouse centromeric sequences because they are not satellite sequences. There is some evidence that this interspacing of $\mathrm{H} 3$ and CENP-A is important to form a three-dimensional binding surface during mitosis with the blocks of CENP-A nucleosomes arranged continuously in three-dimensional space as a sheet where they are exposed for mitotic kinetochore assembly while the H3containing blocks are folded beneath (Marshall et al., 2008). This forms an inner centromeric chromatin domain that contains mostly $\mathrm{H} 3$ nucleosomes and an outer centromeric chromatin face that contains the CENP-A containing nucleosomes.

The epigenetic landscape of centromeric chromatin contains histone modifications consistent with both active and silent chromatin. Chromosome stretching studies in flies and humans have demonstrated that the interspersed centromeric blocks of CENP-A and 
H3 nucleosomes are hypoacetylated but are enriched in dimethylation on H3 Lys-4, 9, and 36 at the inner region of the centromere (Blower et al., 2002; Ribeiro et al., 2010; Sullivan and Karpen, 2004). During mitosis, mitotic kinase Aurora B phosphorylates H3 Ser-10 and CENP-A Ser-7 at both at the inner and outer centromeric chromatin. Additionally, recent work revealed that in humans the CENP-A N-terminal tail, which is quite divergent from the $\mathrm{H} 3$ tail, is post-translationally modified with an N-terminal trimethylation on Gly1 and two phosphorylations on Ser16 and Ser18 (Bailey et al., 2013). The biological significance of these post-translational modifications is still being explored, but preventing phosphorylation on Ser16 and 18 of CENP-A was shown to increase the number of lagging chromosomes observed during anaphase in human cells (Bailey et al., 2013). This epigenetic signature may be important for defining where centromeres are established and propagated, but the mechanism is still not understood.

The alpha-satellite DNA found at human centromeres is neither necessary nor sufficient to specify centromere function. One line of evidence that centromere specification is not DNA-sequence specific is rare, but mitotically stable, neocentromeres are documented to form and function on stretches of DNA containing no centromeric sequences at all (Saffery et al., 2000). Studies in fission yeast and Drosophila have removes endogenous centromeres, thus forcing the cells to form a neocentromere de novo in order to survive. The neocentromeres that form are devoid of centromeric DNA sequences (Ishii et al., 2008; Ogiyama et al., 2013; Williams et al., 1998). Lastly, others and we have shown that stable centromeric chromatin competent for kinetochore formation can be induced at non-centromeric locations in the genome using $\mathrm{LacO} / \mathrm{LacI}$ targeting systems in humans and Drosophila (Barnhart et al., 2011; Chen et al., 2014; 
Gascoigne et al., 2011; Mendiburo et al., 2011). Because of these data, centromeres are thought to be epigenetically specified, and CENP-A is believed to be the epigenetic mark that defines human centromeres. 


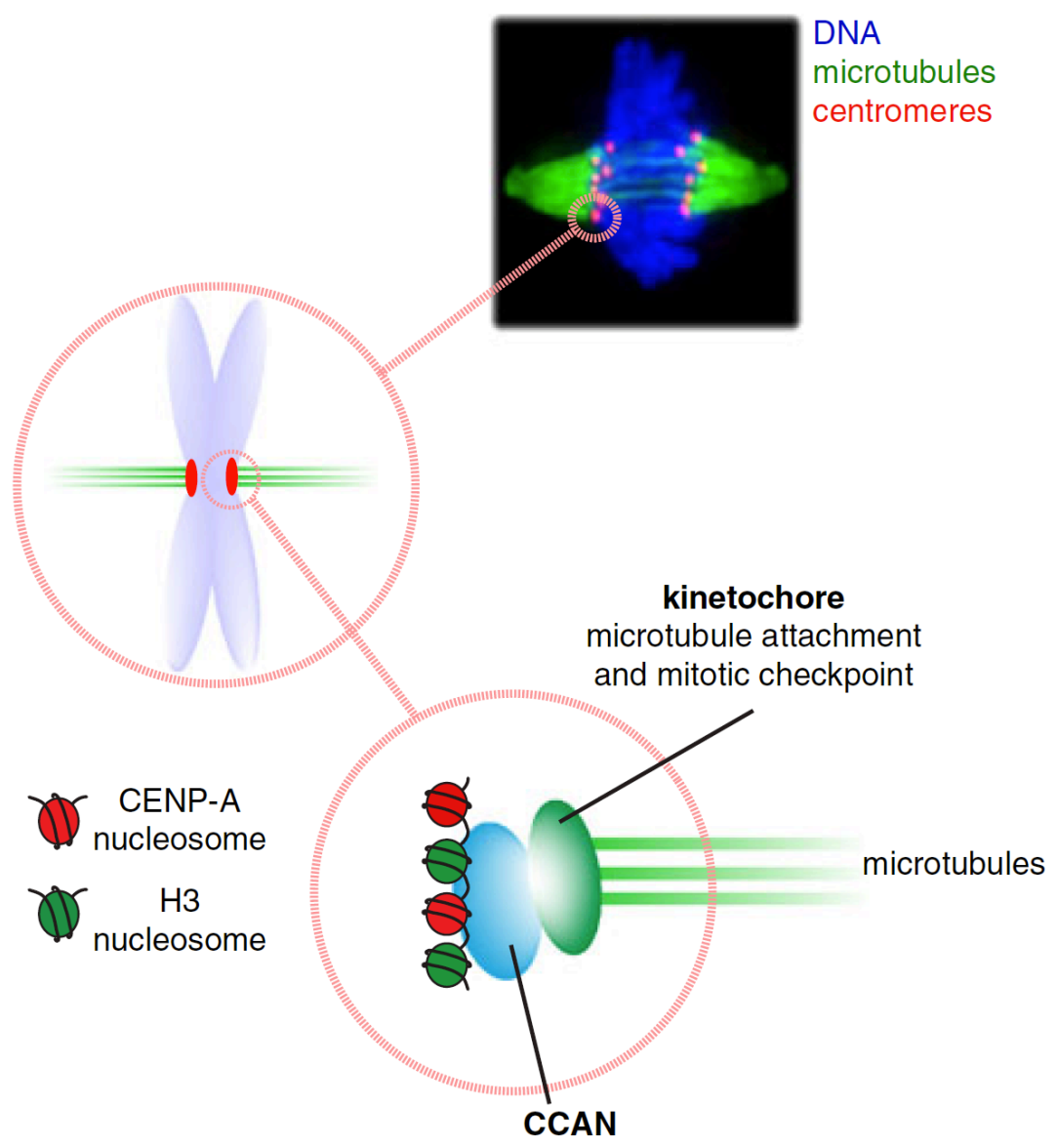

Figure 1-1. Schematic representation of a vertebrate monocentric centromere.

The centromere is a unique chromosomal locus that is epigenetically defined by the presence of nucleosomes containing the histone H3 variant CENP-A. It is the assembly site for the constitutive centromere network throughout the cell cycle. The CCAN then mediates kinetochore attachment during mitosis. The kinetochore interacts with microtubules and mediates sister chromatid separation to each daughter cell during mitosis.

Figure included with permission, (Valente et al., 2012). 


\section{Heterochromatin Environment of Centromere Formation}

One final hallmark of centromeric chromatin is that centromeres from S. pombe to humans are embedded in pericentromeric heterochromatin. In S. pombe, continued transcription and RNAi-mediated processing of these flanking repeats are required for the establishment of the heterochromatic pericentromeric repeats, and the flanking heterochromatin is also required for CENP-A ${ }^{\mathrm{Cnp} 1}$ assembly in the central centromere core (Folco et al., 2008; Volpe et al., 2002). In S. pombe, the need for the RNAi pathway in CENP-A $^{\text {Cnp1 }}$ deposition can be bypassed by directly targeting Clr4 methyltransferase, emphasizing the need for a heterochromatic environment for stable deposition (Kagansky et al., 2009). Higher eukaryotic centromeres in humans and flies are also surrounded by heterochromatin that is marked with $\mathrm{H} 3$ Lys-9-trimethylation by histone methyltransferases Suv39h1\&2, which signals to recruit HP1 (Rice et al., 2003). When CENP-A $^{\text {CID }}$ is over-expressed in Drosophila, it prefers to incorporate ectopically at DNA regions where heterochromatin and euchromatin are adjacent to each other, arguing again that proximity of heterochromatin is an important factor for stable CENP-A deposition (Olszak et al., 2011). Recent studies in fission yeast show H2A.Z incorporates into immature neocentromeres until they establish adjacent heterochromatin, at which point they are stabilized and H2A.Z is evicted (Ogiyama et al., 2013). However, the exact mechanism for why flanking heterochromatin is crucial in stable CENP-A deposition has not been discovered. 


\section{CENP-A as the Centromere-Specific H3 Variant}

Understanding the structure and component features of the $\mathrm{H} 3$ variant CENP-A is essential to determine how CENP-A acts as the epigenetic mark. It is thought that particular features of the CENP-A nucleosome may act as defining characteristics for how centromeric chromatin is recognized and maintained. Several models have been proposed to explain how CENP-A nucleosomes may be differentiated from H3.1 nucleosomes. Data from budding yeast and humans predict an octameric CENP-A nucleosome (Black et al., 2007a; Camahort et al., 2009; Hasson et al., 2013; Miell et al., 2013; Sekulic et al., 2010; Tachiwana et al., 2011). Some studies have suggested these octameric nucleosomes are uniquely compact relative to the histone $\mathrm{H} 3.1$ nucleosome (Black et al., 2007a; Black et al., 2007b; Hasson et al., 2013; Miell et al., 2013; Sekulic et al., 2010).

CENP-A harbors some notable differences from its H3.1 counterpart. The first defining region of the CENP-A nucleosome is the CENP-A targeting domain (CATD). The CATD is the loop 1 and alpha-2 helix of the histone fold of CENP-A. There are several residues in this region that differ from H3.1, and if they are mutated to their H3.1 counterpoints, CENP-A loses centromeric localization, and conversely, an H3.1-CATD chimeric protein is sufficient to rescue CENP-A depletion in human cells (Black et al., 2004; Black et al., 2007b; Shelby et al., 1997). The histone fold of CSE4 is also sufficient for its centromere targeting in S. cerevisiae (Morey et al., 2004). There is also evidence the CATD region of the CENP-A nucleosome is more rigid and compact than its H3.1 counterpart, which may be structurally important given that CENP-A chromatin is the 
anchor for the kinetochore during mitosis (Black et al., 2007a; Black et al., 2004; Jansen et al., 2007). The centromere protein CENP-N has been shown to recognize the CATD on CENP-A in human cells giving additional weight to its importance in identifying the site of centromere assembly (Carroll et al., 2009).

In addition to the CATD, the second distinct characteristic of CENP-A is a shorter $N$-terminal helix containing three fewer residues than H3.1's. This causes a slight unwrapping of the DNA at the octameric CENP-A nucleosome DNA entry/exit point, which has also been shown to cause CENP-A octamers to protect less DNA following MNase digestion than H3.1 octamers (Hasson et al., 2013; Panchenko et al., 2011; Tachiwana et al., 2011). Thirdly, CENP-A contains two additional residues in loop 1 of the histone fold (conserved across all species), and these residues (Arg80/Gly81) induce an accessible bulge that may act as a recognition feature that is somehow important for stable deposition (Sekulic et al., 2010; Tachiwana et al., 2011).

The last defining features of the CENP-A histone are its N and C-terminal histone tails. Histone tails are usually post-translationally modified and describe the epigenetic landscape in which a particular histone exists. Until recently, only one N-terminal modification on CENP-A had been identified in human cells. The kinase Aurora B is known to phosphorylate CENP-A on Ser7 during mitosis in human cells (Zeitlin et al., 2001). Recent studies demonstrated the N-terminal tail of CENP-A is also N-terminally tri-methylated and phosphorylated on Ser16/Ser 18, and removal of these phosphorylations causes chromosome segregation errors (Bailey et al., 2013). The Cterminal tail of CENP-A is composed of six amino acids and is not well conserved from yeast to humans. However, using purified proteins in vitro as well as in Xenopus egg 
extracts, H3.1 nucleosomes containing these six C-terminal CENP-A residues are able to recruit centromere protein $\mathrm{CENP}-\mathrm{C}$, arguing that they may also play a role in addition to the CATD in determining where CCAN members are assembled (Carroll et al., 2010; Guse et al., 2011). However, there is no evidence the C-terminal tail of CENP-A is required to initiate new CENP-A deposition.

There are other more drastic proposed models for how the CENP-A nucleosome is distinct. One model suggests changes in the CENP-A nucleosome composition to include single copies of each of the four core histones (a heterotetramer) in flies and humans (Dalal et al., 2007; Dimitriadis et al., 2010). Another from fission yeast supports a hexameric CENP-A nucleosome that lacks histones $\mathrm{H} 2 \mathrm{~A}$ and $\mathrm{H} 2 \mathrm{~B}$ but includes the non-histone CENP-A/Cse4 chaperone Scm3 (Dalal et al., 2007; Dimitriadis et al., 2010; Mizuguchi et al., 2007; Williams et al., 2009). Additional data from budding yeast confirmed Scm3 evicts $\mathrm{H} 2 \mathrm{~A} / \mathrm{H} 2 \mathrm{~B}$ from Cse4/H4/H2A/H2B octamers (Camahort et al., 2009). Recent quantitative fluorescence measurements suggest an oscillation of the Cse4 copy number in the single Cse4 nucleosome at budding yeast centromeres, cycling from a single copy of Cse4 throughout the cell cycle to two copies of Cse4 specifically in anaphase (Shivaraju et al., 2012). The functional reason for this is unknown. It is important to note the data supporting a heterotetrameric CENP-A nucleosome in Drosophila and humans containing single copies of each histone was generated using atomic force microscopy (AFM), which was recently shown to also detect a reduced height of pre-assembled octameric nucleosomes. This argues the height difference suggested to be due to a heterotetrameric nucleosome may in fact be due to the experimental method of AFM not to any biological reality (Miell et al., 2013). 
Additional evidence supporting an octameric CENP-A nucleosome in budding yeast, flies, and humans comes from data demonstrating the dimerization interface within the CENP-A/Cse4 histone is required for stable deposition (Bassett et al., 2012a; Camahort et al., 2009; Zhang et al., 2012). Finally, it has been suggested that centromeric nucleosomes wrap DNA in a right-handed path around the CENP-A/CenH3 containing histone core, in contrast to the left handed wrapping of the canonical $\mathrm{H} 3$ nucleosome (Furuyama and Henikoff, 2009). This has been refuted in humans with a crystal structure of octameric CENP-A nucleosomes wrapping DNA in a left-handed manner (Tachiwana et al., 2011).

In conclusion, assembled CENP-A nucleosomes have been proposed to exist as octamers, tetrameric hemisomes, as well as hexameric nucleosomes containing two copies of the yeast HJURP homolog, Scm3. Taken together, this suggests CENP-A may exist in a variety of forms between species. However, it is accepted that octameric CENPA nucleosomes exist in abundance at human centromeres and are important for stable CENP-A nucleosome assembly. All of these structural distinctions may contribute specificity to the selective assembly of CENP-A nucleosomes into centromeric loci and to the recruitment of a unique set of proteins to the centromeric chromatin. 


\section{The CCAN: Connecting CENP-A to the Kinetochore}

CENP-A containing chromatin is the binding site for a set of proteins termed the CCAN - the Constitutive $\underline{\text { Centromere }}$ Associated Network. This growing set of 16 proteins is constitutively associated with centromeric chromatin and serves as the interface between centromeric chromatin and the kinetochore during mitosis. In addition, there are established roles for a few of these CCAN members in new CENP-A replenishment each cell cycle indicating they contribute to the unique character that identifies centromeric chromatin. Within the set of 16 CCAN proteins, there are distinct groups of CENP's that co-purify together and are often interdependent with each other for centromeric localization. In addition to the sub-grouping, there is a hierarchy of recruitment for each sub-group building out from the CENP-A nucleosome associated

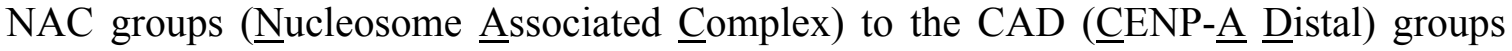
that interact with the outer kinetochore and even spindle microtubules. Many of the CCAN proteins are conserved from yeast to humans, however some organisms like Drosophila and C. elegans appear to completely lack CCAN proteins other than CENP-C (Perpelescu and Fukagawa, 2011).

Some of the earliest identified CENP's (CENP-A, CENP-B, and CENP-C) were detected by their localization to the centromere region and through their recognition by antibodies in the sera of patients with Sclerodactyly and Telangiectasia (CREST) syndrome (Earnshaw and Rothfield, 1985; Moroi et al., 1980). The bulk of the CCAN was later found as proteins that co-purified with CENP-A chromatin in human tissue culture cells (Foltz et al., 2006; Obuse et al., 2004). A parallel study co-identified CCAN 
members through purification of two already identified CENP's, CENP-H and CENP-I, from chicken cells (Okada et al., 2006). Biochemical purifications and protein depletion studies have assigned the CENP's into sub-groups, allowing distillation of the many CENP's into more functionally specific groups (reviewed in (Perpelescu and Fukagawa, 2011). The groups relevant to the work presented here are the CENP-C group, the CENP$\mathrm{T} / \mathrm{W} / \mathrm{S} / \mathrm{X}$ group, and the CENP-H/I/K/L/M/N group. The additional group, composed of $\mathrm{CENP}-\mathrm{O} / \mathrm{P} / \mathrm{Q} / \mathrm{R} / \mathrm{U}$, is required for maintaining and building quality kinetochoremicrotubule interactions, is necessary for recruiting the mitotic kinase Plk1 to kinetochores, and itself requires the CENP-H/I/K complex to properly localize to centromeres (Hori et al., 2008b; Izuta et al., 2006; Okada et al., 2006).

While all the CCAN proteins co-purify with CENP-A, only CENP-C and CENP$\mathrm{N}$ are known to bind directly to the CENP-A nucleosome (Carroll et al., 2010; Carroll et al., 2009). CENP-C is an integral protein to both the CENP-A assembly pathway and the kinetochore architecture. Its role in CENP-A assembly will be discussed in the CENP-A deposition pathway section. CENP-C ${ }^{\text {aa422-537 }}$ can bind directly to CENP-A nucleosomes in vitro consistent with it purifying closely with CENP-A chromatin from in vivo purifications (Carroll et al., 2010; Foltz et al., 2006). In human cells CENP-N, though it does not physically interact with CENP-C, binds specifically to the CATD of the CENPA nucleosomes and acts in a dual recognition pathway along with CENP-C to specifically identify centromeric chromatin (Carroll et al., 2010; Carroll et al., 2009). CENP-N depletion reduces the ability of human and fission yeast cells to load new CENP-A/Cnp1 (Carroll et al., 2009; Hayashi et al., 2004). Additionally, using single molecule fluorescence distance measurements (K-SHREC), CENP-C was localized the closest to 
the CENP-A chromatin of any of the CCAN or kinetochore proteins examined (Wan et al., 2009). It is also known the N-terminus of human and fly CENP-C is required to recruit the Mis12 complex, a member to the microtubule-binding KMN network, to kinetochores (Gascoigne et al., 2011; Przewloka et al., 2011; Screpanti et al., 2011). CENP-C binds to DNA and RNA, both of which have been shown to play a role it its centromeric recruitment (Du et al., 2010; Wong et al., 2007). Along with CENP-T, CENP-C and CENP-N are cornerstone CENP's required for centromere and kinetochore assembly with interactions spanning from the CENP-A nucleosome out to the KMN network at the kinetochore-microtubule binding interface.

The CENP-T/W/S/X group is also directly required to recruit the KMN network to kinetochores as it binds to Ndc80 complex via its N-terminus (Gascoigne et al., 2011; Nishino et al., 2013). CENP-T depletion causes loss of the CENP-N/M/H/I/K from centromeres (Foltz et al., 2006). The CENP-T/W/S/X CCAN group is unique in that each member contains a histone fold domain. CENP-W closely associates with CENP-T via its histone fold domain. Interestingly, CENP-T/W both co-purify more closely with the H3 nucleosomes that are interspersed with CENP-A at the centromere than with CENP-A (Hori et al., 2008a; Ribeiro et al., 2010). The C-terminal histone fold domain of CENP-T is connected to the N-terminal Ndc80-binding portion of the protein by a flexible linker, and immuno-EM studies revealed this linker allows CENP-T to stretch like a spring while kinetochores are under tension from microtubules (Suzuki et al., 2011). The CENP$\mathrm{S} / \mathrm{X}$ proteins form a heterotetramer with CENP-T/W in vitro and also positively supercoil DNA (Nishino et al., 2012; Takeuchi et al., 2014). This striking arrangement bears many similarities to a nucleosome-like particle and was suggested to be a centromere-specific 
alternative nucleosome. CENP's-T/W/S/X turn over each cell cycle and are newly deposited at the end of each S-phase (Dornblut et al., 2014; Prendergast et al., 2011). Given these proteins are turned over right before mitosis, this suggests the CENP$\mathrm{T} / \mathrm{W} / \mathrm{S} / \mathrm{X}$ complex contributes, perhaps through alternative nucleosome formation, to creating a unique chromatin environment conducive for CENP-A deposition in the upcoming G1 (Dornblut et al., 2014; Foltz and Stukenberg, 2012).

Lastly, the CENP-H/I/K CCAN complex is recruited distally from the CENP-A chromatin with respect to CENP-C and CENP-T. CENP-N, CENP-C, and CENP-T are required to recruit the CENP-H/I/K complex to centromeres (Carroll et al., 2010; Carroll et al., 2009; Foltz et al., 2006). CENP-H/I/K itself is also required to recruit many other CCAN components as well as KNL1 (Cheeseman et al., 2008). One group found CENP$\mathrm{H} / \mathrm{I} / \mathrm{K}$ is required for new CENP-A loading, and this is proposed to occur through recruitment of the FACT subunit, CHD1 (Okada et al., 2009). The CENP-H/I/K complex is important for regulating kinetochore-microtubule attachments. The complex displays dynamic enrichment at kinetochores specifically associated with growing microtubule ends (Amaro et al., 2010). CENP's-H/I/N are also known to recruit the spindle assembly checkpoint protein Mad2 to unattached kinetochores under conditions where Aurora B is comprised (Matson et al., 2012).

The interactions within the CCAN hierarchy, the interdependencies of their localization, and their influence on CENP-A deposition, centromere recognition, and kinetochore assembly are intricate. Many studies have contributed to building to current view of what these proteins are doing at the centromere, and only the seminal findings were discussed here. However, many of the CCAN proteins still do not have ascribed 
functions, so there is much to be learned about this macromolecular complex. A simplified model of the CCAN sub-groups and their relative location within the centromere-kinetochore interface is depicted in Figure 1-2. In conclusion, the CCAN and the CENP-A nucleosome work in concert to define centromeric chromatin and allow it to be specifically recognized and inherited throughout the lifetime of a cell. 


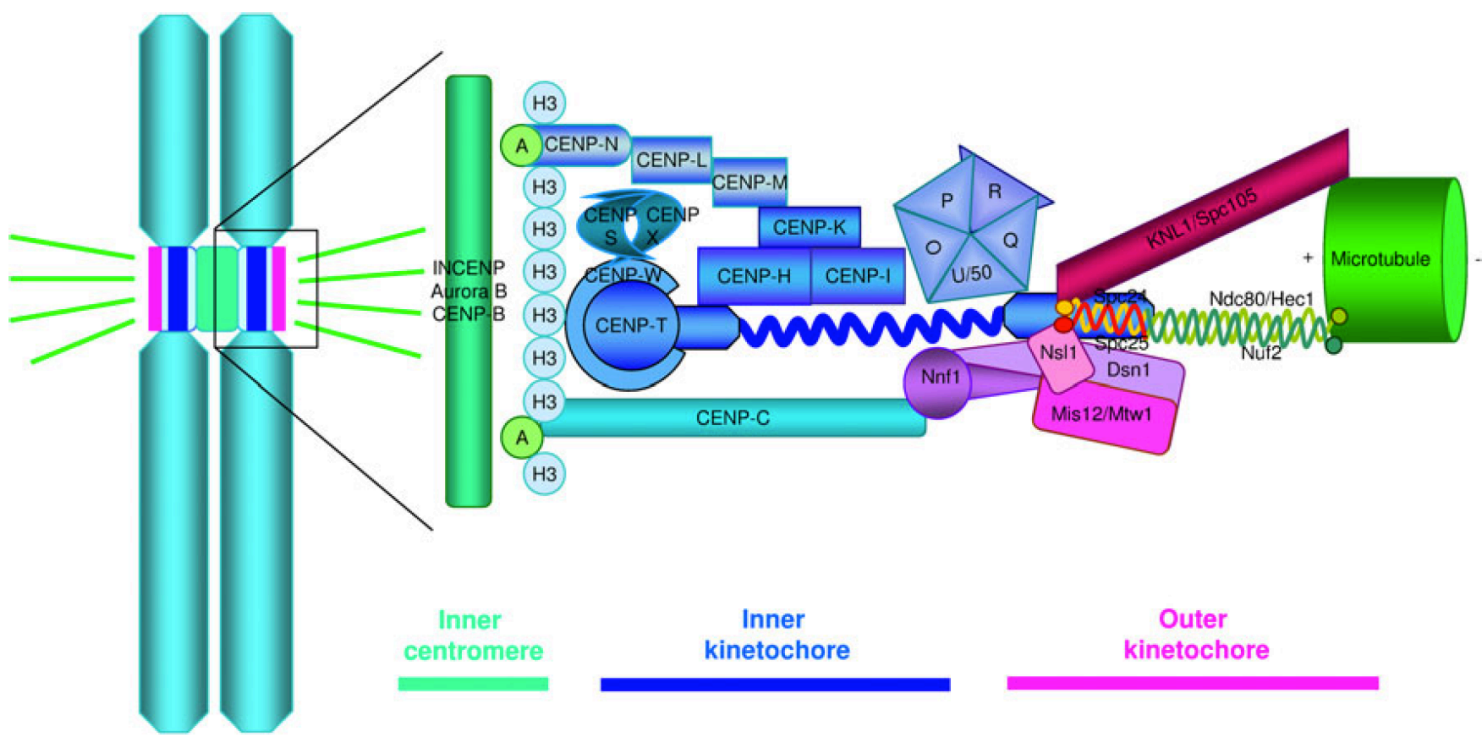

Figure 1-2. Molecular organization of the vertebrate constitutive centromere network and its interface with the kinetochore.

This schematic represents a vertebrate sister chromatid pair during mitosis with an emphasis on displaying the various sub-groupings of the CCAN members. The vertebrate centromere and kinetochore have three main regions, the inner centromere, the inner kinetochore, and the outer kinetochore. CENP-A nucleosomes interspersed with H3 nucleosomes provide the base for CCAN assembly. CENP-C and CENP-N directly bind to CENP-A nucleosomes. CENP-T co-purifies more closely with $\mathrm{H} 3$ nucleosomes at the centromere. CENP-C and CENP-T both bind to the KMN network, which interfaces the CCAN to microtubules. CENP-H///K are involved in regulating kinetochore-microtubule attachments.

Figure included with permission, (Perpelescu and Fukagawa, 2011). 


\section{$\underline{\text { Artificial Centromere Formation }}$}

Ectopic centromere creation and manipulation is a useful tool for studying the sufficiency of various proteins for CENP-A deposition and centromere formation in yeast, flies, and humans. Cell lines containing stably integrated LacO tandem repeats have been used to tether individual centromere components using LacI. This method is advantageous because it allows one to assay individual proteins separately from their usual context at centromeric $\alpha$-satellite sequences. Chapter II of this work will demonstrate the sufficiency of HJURP for de novo CENP-A chromatin formation and subsequent kinetochore assembly in human cells using this system. Other groups have also employed this system for assaying centromere and kinetochore formation in flies and plants (Chen et al., 2014; Mendiburo et al., 2011; Teo et al., 2013). Tethering LacICENH3/CID in Drosophila cells is sufficient to epigenetically establish a kinetochore at a non-centromeric site and induce epigenetic recruitment and spreading of endogenous CENH3/CID to the non-centromeric site in subsequent generations (Mendiburo et al., 2011). Recently, similar results were demonstrated by tethering the CENH3/CID assembly factor, CAL1, or CENP-C to non-centromeric chromatin in Drosophila cells (Chen et al., 2014). Lastly, this system has been used to assemble a de novo kinetochore without the underlying CENP-A chromatin by tethering CENP-C and CENP-T together at a LacO array in human cells. These de novo kinetochores are not heritable, however, because they lack underlying CENP-A chromatin (Gascoigne et al., 2011). The successful assembly of these de novo centromeres and kinetochores at non-centromeric sites reinforces the dispensable role of DNA sequence in centromere formation. 
Another method to assay de novo centromere formation is to remove the endogenous centromere thus forcing the cell to induce neocentromere formation. One system in fission yeast flanks the endogenous centromeres with loxP sites so that following expression of Cre recombinase the centromere is excised. Interestingly, the rare induced neocentromeres most frequently formed just adjacent to telomeres and were specifically absent from highly transcribed gene clusters in this region (Ishii et al., 2008). Later studies using this same system revealed the importance of establishing adjacent heterochromatin for the neocentromeres' stability (Ogiyama et al., 2013). Centromere removal to induce neocentromere formation has also been successfully applied in vertebrate cells. Chicken DT-40 cells were engineered with the $\mathrm{Z}$ chromosome flanked with $\operatorname{loxP}$ sites and a LacO array present on the same chromosome. Thus, following endogenous centromere removal by Cre recombinase, LacI fused rescue proteins were assayed for their ability to initiate de novo centromere formation at the $\mathrm{LacO}$ array. Interestingly, tethering HJURP, the C-terminus of CENP-C, or CENP-I induced CENP-A containing chromatin and subsequent kinetochore formation. Importantly, once CENP-A chromatin was established, the LacI fusion seed proteins became dispensable for the maintenance of this de novo kinetochore. In contrast, tethering CENP-T or the Nterminus of CENP-C could also rescue chromosome loss by initiating de novo kinetochore formation, but these kinetochores required the constant presence of the LacI seed proteins for stability because they lacked underlying CENP-A chromatin (Hori et al., 2013). This same group also induced centromere excision in DT-40 cells lacking the LacO array, thereby forcing the cell to form a de novo without any LacI seed proteins. They observed that $76 \%$ of the time, neocentromeres formed in the regions just adjacent 
to the original excised centromere. Using ChIP-Seq they were able to demonstrate that small amounts of non-kinetochore CENP-A is incorporated into $2 \mathrm{Mb}$ regions flanking the endogenous centromere that can act to seed a new centromere in case the endogenous one is lost. This is a very interesting concept, but it is still not clear how this dormant, non-kinetochore CENP-A becomes active and competent to seed neocentromere formation following endogenous centromere removal. In conclusion, de novo centromere formation assays have highlighted the necessity of CENP-A chromatin, from yeast to vertebrate cells, in epigenetically deciding where a centromere will be established and propagated. They have additionally given insight into the hierarchy of recruitment of the various CCAN and kinetochore proteins. 


\section{The CENP-A Deposition Pathway}

S-phase players

CENP-A deposition is cell cycle regulated. Unlike canonical H3 nucleosomes, CENP-A deposition is uncoupled from DNA replication. In human cells, new CENP-A is synthesized in S-phase similarly to H3.1; however, CENP-A protein levels do not peak until G2 making newly synthesized CENP-A unavailable for deposition concurrent with replication (Shelby et al., 2000; Shelby et al., 1997). Instead, human cells proceed into G2 and mitosis with roughly half the full complement of CENP-A at their centromeres (Dunleavy et al., 2011; Jansen et al., 2007). It is unknown how CENP-A nucleosomes transit the replication fork and what proteins are involved in this, but this is an area of current study.

The dynamics of CCAN components CENP-T/W/S/X, CENP-N, and CENP-C during S-phase may also play a role in marking centromeric chromatin during S-phase, G2, and mitosis when CENP-A levels are reduced. The histone-fold domain containing CENP's-T/W/S/X turn over each cell cycle and are newly deposited at the end of each S-

phase (Dornblut et al., 2014; Prendergast et al., 2011). CENP's-N and C both dynamically exchange at the centromere throughout the cell cycle, but each becomes more stably associated during S-phase (Hellwig et al., 2011; Hemmerich et al., 2008). The stabilization of CCAN components during a time when centromeric CENP-A levels are reduced is likely important for maintaining easily identifiable centromeric chromatin when the time comes to deposit new CENP-A in early G1. 


\section{Timing}

In order to maintain centromere identity, CENP-A dilution in S-phase must be countered by new CENP-A deposition each cell cycle. CENP-A deposition is tightly regulated by the cell cycle and has three main steps: initiation or preparation of the underlying chromatin, deposition of CENP-A into the chromatin, and maintenance and maturation of the CENP-A nucleosomes (Stellfox et al., 2013). Specific proteins carry out each of these steps at specific times during the cell cycle resulting in high temporal and spatial regulation of centromeric chromatin assembly (Figure 1-3).

In humans, Xenopus CSF extracts, and Drosophila, new CENP-A is loaded at centromeres in late anaphase or early G1 just after the cell exists mitosis (Bernad et al., 2011; Jansen et al., 2007; Mellone et al., 2011; Schuh et al., 2007). Because CENP-A deposition occurs only during this specific window of time, the centromeric localization of the proteins involved in CENP-A deposition must be tightly cell cycle regulated. The cyclins and the cyclin-dependent kinases (Cdk's) regulate the cell's progression through the cell cycle. During the S, G2, and M phases of the cell cycle, cyclin E/Cdk2 (S phase only), cyclin A (activates Cdk2), and cyclin B (activates Cdk1) are active. Cyclin B/Cdk1 reach maximal activity at the $\mathrm{G} 2 / \mathrm{M}$ transition and are the primary players involved in mitotic progression (Reviewed in (Glover, 2012). Their activity remains high until the spindle assembly checkpoint turns off, at which point the cyclins are degraded by the anaphase promoting complex (APC). The cell then progresses into anaphase with low Cdk activity (Stellfox et al., 2013). The first known step in the CENP-A deposition pathway occurs at the end of anaphase in human cells, so it is logical that regulation by Cdk's may control when the CENP-A deposition machinery localizes to centromeres. 
Consistent with this, inhibition of Cdk's in HeLa and chicken cells causes premature loading of the CENP-A deposition machinery and early CENP-A loading during G2 (Silva et al., 2012). Recently, it was reported that the CENP-A chaperone HJURP is phosphorylated by Cdk1, and this phosphorylation weakens HJURP's interaction with Mis18 $\beta$, thus supporting Cdk activity preventing premature centromeric recruitment of the CENP-A deposition machinery (Wang et al., 2014). Similarly, Scm3 (HJURP homolog in fission yeast) is phosphorylated at a putative Cdk consensus site (Pidoux et al., 2009). Finally, the protein levels of the CENP-A deposition machinery and CENP-A itself peak in S-phase and G2 (Dunleavy et al., 2009; Foltz et al., 2009; Shelby et al., 2000; Shelby et al., 1997). The fact that they do not localize until early G1 indicates that assembly is actively prevented until mitotic exit.

Unlike the higher eukaryotes mentioned above where CENP-A is deposited in anaphase or early telophase/G1, CENP-A deposition in budding yeast occurs during DNA replication. Photobleaching experiments in budding yeast revealed the single CENP-A/Cse4-containing nucleosome is completely replaced each cell cycle concurrent with DNA replication (Pearson et al., 2004). At regional centromeres in fission yeast, CENP-A/Cnp1 loading occurs primarily in S-phase and to a lesser extent in G2. S-phasedependent deposition requires the transcription factor Ams2 (Takayama et al., 2008). Additionally, histone-binding protein $\operatorname{Sim} 3$ is required for replication independent deposition of Cnp1 is fission yeast (Dunleavy et al., 2007). Lastly, CENP-A/CENH3 deposition in plants occurs in late G2 (Lermontova et al., 2006). In conclusion, the timing of CENP-A deposition varies between model organisms. The timing of CENP-A deposition and the players involved is summarized in Table 1-1. 
Figure 1-3: The CENP-A deposition cycle in human cells.

CENP-A deposition is tightly regulated by the cell cycle. During S-phase, existing CENP-A is divided between the daughter strands of the replicated centromeric chromatin. The cell progresses into mitosis with half its full complement of CENP-A. Upon mitotic exit, cyclins are rapidly degraded and Cdk activity drops. The Mis 18 complex localizes to centromeres and licenses them for deposition. HJURP is recruited with new CENP-A/H4 in tow, and new CENP-A is loaded. Later in G1, remodeling factors Rsf1 and CHD1 localize to centromeres and are required to fully incorporate the CENP-A nucleosomes into the centromeric chromatin. In late G1, MgcRacGAP and Ect2 localize to centromeres and stabilize the new CENP-A nucleosomes by a mechanism requiring GTP cycling activity.

Figure included with permission, (Nechemia-Arbely et al., 2012). 


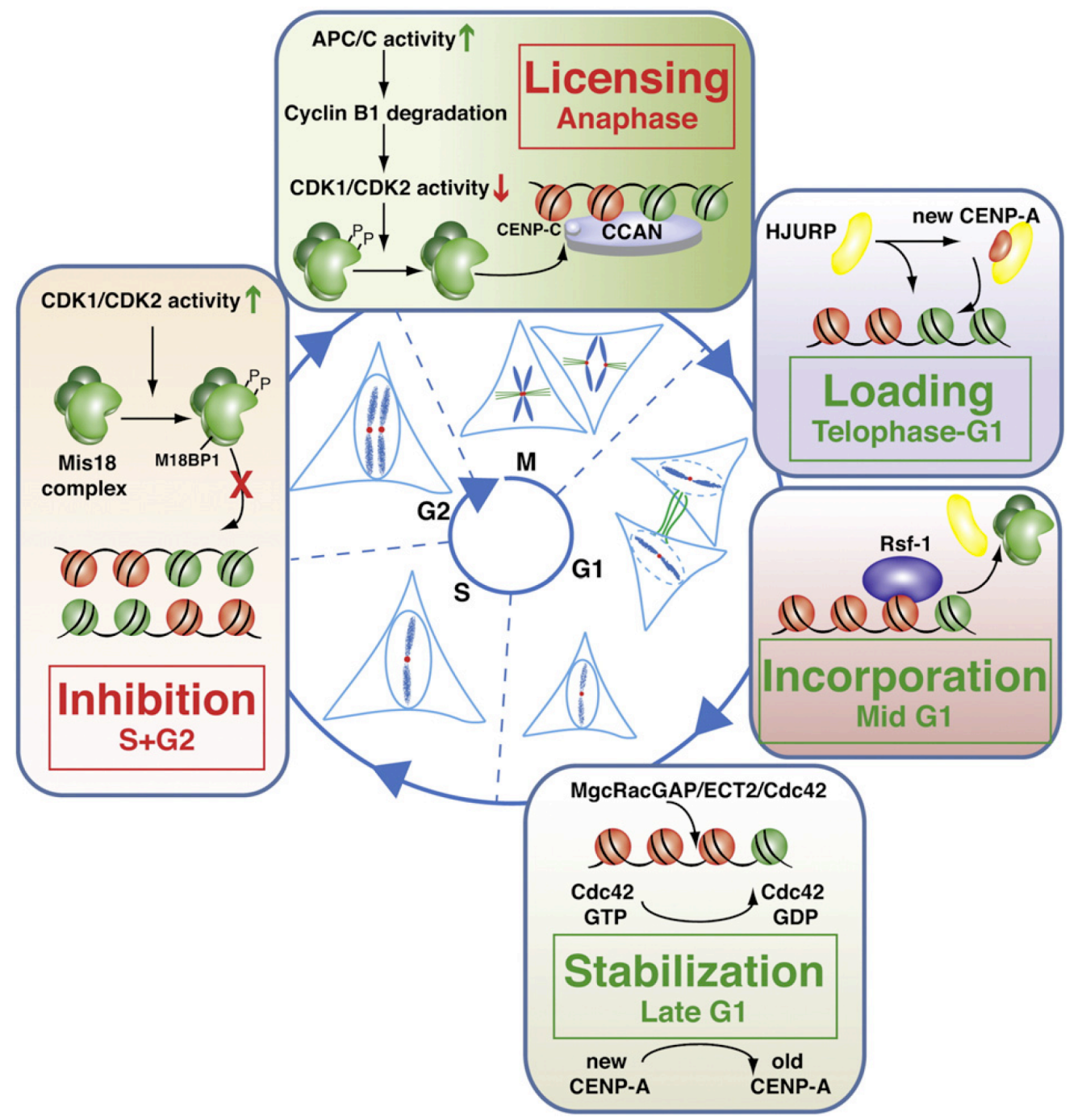




\begin{tabular}{|c|c|c|c|c|}
\hline Organism & $\begin{array}{l}\text { Timing of } \\
\text { CENP-A } \\
\text { Deposition }\end{array}$ & Mis18 & $\begin{array}{l}\text { HJURP/ } \\
\text { Scm3 or } \\
\text { Cal1 }\end{array}$ & $\begin{array}{l}\text { References } \\
\text { for Timing }\end{array}$ \\
\hline Human cells & $\begin{array}{l}\text { Late anaphase- } \\
\text { early G1 }\end{array}$ & $\begin{array}{l}\text { Mis18 } \\
\text { Mis18 } \\
\text { Mis18BP1 }\end{array}$ & HJURP & Jansen, 2007 \\
\hline Xenopus & $\begin{array}{l}\text { Late anaphase- } \\
\text { early interphase }\end{array}$ & $\begin{array}{l}\text { Mis18 } \\
\text { Mis18 } \\
\text { Mis18BP1 }\end{array}$ & xHJURP & $\begin{array}{l}\text { Moree, } 2011 \\
\text { Bernad, } 2011\end{array}$ \\
\hline Mouse & & $\begin{array}{l}\text { Mis18 } \\
\text { Mis18 } \\
\text { Mis18BP1 }\end{array}$ & HJURP & \\
\hline Zebrafish & & 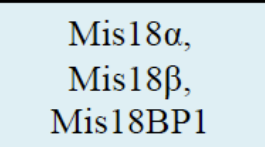 & HJURP & \\
\hline Drosophila & Anaphase & -- & CAL1 & $\begin{array}{c}\text { Schuh, } 2007 \\
\text { Mellone, } 2011\end{array}$ \\
\hline Arabidopsis & Late G2 & Mis 18 & -- & $\begin{array}{c}\text { Lermontova, } \\
2006\end{array}$ \\
\hline $\begin{array}{c}\text { Chicken } \\
\text { DT40 cells }\end{array}$ & $\begin{array}{l}\text { Late anaphase- } \\
\text { early G1 }\end{array}$ & $\begin{array}{c}\text { Mis18 } \alpha \text {, } \\
\text { Mis18 }, \\
\text { Mis18BP1 }\end{array}$ & HJURP & Silva, 2012 \\
\hline C. elegans & Mitosis & Knl2/Mis18BP1 & -- & $\begin{array}{l}\text { Maddox, } 2007 \\
\text { Oegema, } 2001\end{array}$ \\
\hline S. pombe & $\begin{array}{c}\text { S-phase, } \\
\text { Some G2 }\end{array}$ & Mis 18 & $\mathrm{Scm} 3$ & $\begin{array}{l}\text { Takayama, } 2008 \\
\text { Dunleavy, } 2007\end{array}$ \\
\hline S. cerevisiae & S-phase & -- & $\mathrm{Scm} 3$ & Pearson, 2007 \\
\hline
\end{tabular}

Table 1-1: Timing of CENP-A loading across species.

Chart summarizes the timing of CENP-A deposition across various model organisms.

References are for papers identifying this timing. The presence of the Mis18 proteins and HJURP/Scm3 is also indicated for each organism. (--) indicates absent. Blank cells indicate not investigated. 
Players involved in priming: CENP-C and the Mis 18 Complex

\section{The Mis 18 Complex}

Mis18 was originally identified in fission yeast in a screen for proteins that caused chromosome missegregation (Hayashi et al., 2004). Mis16 (RbAP46/48 in humans) was also found to interact with Mis18. Mutation of either Mis16 or Mis18 resulted in decreased Cnp1 levels at centromeres (Hayashi et al., 2004) providing evidence that these proteins were part of the CENP-A deposition pathway. In humans, there are two Mis18 homologs, Mis18 $\alpha$ and Mis18 $\beta$, as well as a third binding partner called Mis18BP1. Depletion of any one of the three complex members prevents the whole complex from localizing to centromeres (Stellfox et al. Under Review 2014); (Fujita et al., 2007). Mis18 $\alpha$ and Mis18 $\beta$ interact as a heterotetramer separate from Mis18BP1 in a pre-

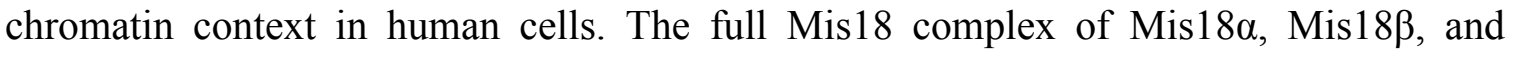
Mis18BP1 only comes together on chromatin (Nardi et al. Unpublished results, 2014). Interestingly, RbAp46/48 were also found to associate with the Mis 18 complex in human cells, and their depletion also affects CENP-A loading. Because RbAP46/48 is a common member of many chromatin remodeling complexes, its effect on CENP-A loading may be attributable to any number of complexes affected by RbAP46/48 depletion so is difficult to interpret (Dunleavy et al., 2009; Fujita et al., 2007). Some species, C. elegans for example, only have a Mis18BP1 homolog but no Mis18 $\alpha$ or Mis18 $\beta$ (Maddox et al., 2007). Drosophila does not have any Mis 18 homologs at all. Despite the variability in conservation, the Mis 18 complex is absolutely required for CENP-A deposition in human cells. 
The recruitment of the Mis18 complex to centromeres in S. pombe, X. laevis, $C$. elegans, and humans initiates the CENP-A deposition pathway. In Xenopus and humans, the Mis 18 proteins localize to the centromeres just after the cell exits mitosis, immediately prior to CENP-A deposition (Fujita et al., 2007; Moree et al., 2011). In fission yeast and Arabidopsis, Mis18 occupies the centromere throughout the cell cycle except during a short window in mitosis (Hayashi et al., 2004; Lermontova et al., 2013; Pidoux et al., 2009; Williams et al., 2009). In fission yeast, Xenopus, and humans, Mis18 is required to recruit the CENP-A/Cnp1 chaperone HJURP/Scm3 to centromeres; and therefore, Mis18 depletion leads to a loss of CENP-A at centromeres (Barnhart et al., 2011; Fujita et al., 2007; Hayashi et al., 2004; Moree et al., 2011; Pidoux et al., 2009; Williams et al., 2009). Similarly, depletion of the C. elegans Mis18BP1 homolog, KNL2, is also sufficient to disrupt CENP-A deposition (Maddox et al., 2007).

The reason why Mis18 is required for CENP-A deposition is still unclear. One possibility is the Mis 18 complex may epigenetically prime centromeric chromatin to make it compliant for CENP-A deposition. In fission yeast, Mis18 temperature sensitive mutants show increased acetylation at their centromeric repeats (Hayashi et al., 2004). However, data in human cells shows a contradictory phenotype in that treating cells with the HDAC inhibitor, trichostatin A, rescues a Mis18 depletion phenotype and restores CENP-A to centromeres (Fujita et al., 2007). Mis18 $\alpha$ has recently been shown to interact with DNMT3A and DNMT3B and eliminating this interaction by depleting Mis18 $\alpha$ led to a reduction in methylation of centromeric chromatin and decreased centromeric CENP-A levels, though DNMT3 knockouts are viable (Gopalakrishnan et al., 2009; Kim et al., 2012). Additionally, mouse embryonic fibroblasts lacking Mis18a have a 
chromatin condensation defect in prometaphase arguing that Mis18 $\alpha$ may be able to condense chromatin in some way (Kim et al., 2012). Finally, the interaction of the Mis 18 proteins with Mis16/RbAP46/48 discussed above suggests they may play a role in chromatin remodeling at the centromere. All these interactions put Mis18 in a good position to alter the epigenetic environment at the centromere and prime it for successful HJURP/Scm3 recruitment then CENP-A deposition.

Mis18 may also influence CENP-A deposition by serving as the binding site for HJURP/Scm3 at the centromere. In the original Mis18 and CENP-A purifications from fission yeast and human cells, a physical interaction between CENP-A/Cnp1 or HJURP/Scm3 and Mis18 was never detected (Dunleavy et al., 2009; Foltz et al., 2009; Fujita et al., 2007; Hayashi et al., 2004). Later studies in fission yeast revealed that Scm3 could bind to Mis18 in vitro (Pidoux et al., 2009). Recently, however, Wang et al. and our lab have demonstrated in vitro and in vivo interactions between HJURP and Mis18 $\alpha /$ Mis18 $\beta$ in human cells (Nardi et al. Unpublished results 2014), (Wang et al., 2014). Because the interactions between the Mis 18 proteins and either CENP-A/Cnp1 or HJURP/Scm3 have not been robustly detected in vivo, this is consistent with these interactions being highly regulated and short-lived in the cell. In line with this, Mis18 and HJURP only co-localize at the centromere in early G1 cells (Foltz et al., 2009).

Together, these data demonstrate centromeric recruitment of the Mis18 proteins at the end of mitosis in mammalian cells is a critical regulatory step in preparing centromeric chromatin for CENP-A deposition. The mechanistic details of how Mis18 acts at centromeric chromatin to facilitate CENP-A deposition continue to be investigated. Importantly for work discussed here, the Mis18 complex is required to 
recruit HJURP, which results in CENP-A deposition in human cells (Barnhart et al., 2011).

CENP-C

It is thought that one of the ways regional centromeres spatially direct CENP-A recruitment and deposition is through CENP-C. Centromere protein C (CENP-C) is a member of the CCAN and is known to directly bind to CENP-A nucleosomes (Carroll et al., 2010; Guse et al., 2011). Unique from other characterized members of the CCAN, it has been shown to play a central role in determining where CENP-A deposition occurs in higher eukaryotes. CENP-C has also been shown to be required for CENP-A deposition, further emphasizing its importance in the CENP-A deposition pathway (Carroll et al., 2010; Dambacher et al., 2012; Erhardt et al., 2008). Interactions between CENP-C and the Mis18 complex protein Mis18BP1 have been demonstrated in humans, Xenopus, and mice (Stellfox et al. Under Review 2014); (Dambacher et al., 2012; Moree et al., 2011). In line with CENP-C spatially regulating CENP-A deposition, in Drosophila where there are no Mis18 proteins, the CENP-A chaperone and deposition protein CAL1 has also been shown to physically interact with CENP-C (Chen et al., 2014; Erhardt et al., 2008). Therefore, a requirement for the CENP-A deposition machinery to recognize CENP-C, thus ensuring CENP-A is deposited in the correct location, seems to be conserved even in species that lack Mis18BP1.

Depleting CENP-C leads to impaired but not abolished Mis18BP1 recruitment to centromeres in mouse cells while in Xenopus, the isoform of Mis18BP1 that localizes to centromeres during G1 is not affected by CENP-C knockdown (Dambacher et al., 2012; Moree et al., 2011). The current model has been that CENP-C recruits Mis18BP1, which 
then recruits its complex members Mis $18 \alpha$ and Mis $18 \beta$ to centromeres, thus initiating the CENP-A deposition pathway. Recent work from our lab demonstrates that this model is overly simplified and that while Mis18BP1 is targeted to centromeres via direct binding to CENP-C, its robust recruitment to centromeres also requires binding to Mis18 $\alpha$. Mis $18 \alpha$ is bound to Mis $18 \beta$, which also binds to CENP-C in a cell cycle dependent manner, which generates full Mis18 complex recruitment (Stellfox et al., Under Review 2014). Additionally, targeting CENP-C to a non-centromeric locus is not sufficient to initiate CENP-A chromatin formation at that site (Gascoigne et al., 2011). So while CENP-C does play a central role in recruiting the CENP-A deposition machinery, it is not solely its interaction with Mis18BP1 that generates full Mis18 complex recruitment to the centromere. 
Players Involved in CENP-A Loading

HJURP

The unique timing and deposition pattern of human CENP-A suggests that it has its own unique set of chromatin assembly factors. Affinity purifications of prenucleosomal CENP-A identified HJURP ( which specifically associated with CENP-A and not H3.1 (Dunleavy et al., 2009; Foltz et al., 2009). HJURP was originally identified prior to these studies as an over-expressed protein in non-small cell lung carcinomas (Kato et al., 2007). HJURP received its name because it was shown to bind to synthetic Holliday junctions in vitro, and in this original paper, HJURP was noted to localize to nucleoli, bind to rDNA by ChIP, and suggested to play a role in the homologous recombination repair pathway for DNA double-stranded breaks, but its role at the centromere was not investigated (Kato et al., 2007). HJURP localizes to nucleoli throughout G1, S-phase, and G2, but it is most enriched there during S-phase (Dunleavy et al., 2009). The specific co-purification of HJURP with prenucleosomal CENP-A suggested it may have chaperone activity, and investigation revealed HJURP localized to centromeres during a short window during early G1 just after cells complete anaphase, the same time new CENP-A is deposited (Dunleavy et al., 2009; Foltz et al., 2009; Jansen et al., 2007). Depletion of HJURP resulted in a loss of CENP-A at centromeres (Dunleavy et al., 2009; Foltz et al., 2009). SNAP-labeling to specifically follow the pool of newly synthesized CENP-A revealed that HJURP depletion completely blocked the loading of new CENP-A into centromeric chromatin, demonstrating HJURP as the CENP-A chaperone (Foltz et al., 2009). 
In addition to HJURP, the prenucleosomal CENP-A purifications identified that Npm1 and RbAP46/48 also interacted with pre-nucleosomal CENP-A (Dunleavy et al., 2009; Foltz et al., 2009). RbAP48 was also shown to co-purify with prenucleosomal CENP-A/CID in Drosophila (Furuyama et al., 2006). Depletion of either RbAP46 or 48 also reduced CENP-A/Cnp1 levels at centromeres in fission yeast and human cells as well decreased HJURP protein levels in human cells. Though, since these proteins are components of many chromatin remodeling complexes, their knockdown likely affects multiple pathways in the cell (Dunleavy et al., 2009; Hayashi et al., 2004). Depletion of Npm1 did not have any obvious effect on CENP-A levels at centromeres, though Npm1 is an abundant protein so may not have been effectively depleted (Dunleavy et al., 2009).

In S. cerevisiae and S. Pombe, the protein Scm3 functions analogously in place of HJURP in that it is required for $\mathrm{Cse}^{\text {S.c. }} / \mathrm{Cnp}^{\text {S.p. }}$ maintenance and assembly at centromeres (Camahort et al., 2007; Camahort et al., 2009; Mizuguchi et al., 2007; Pidoux et al., 2009; Stoler et al., 2007; Williams et al., 2009). The primary sequences of HJURP and Scm3 are divergent, but they both share a conserved 62 amino acid CENP-A binding region ( $\mathrm{Scm} 3$ domain) in their $\mathrm{N}$-termini despite differing strikingly at their $\mathrm{C}$ termini (Sanchez-Pulido et al., 2009). Even between fungi, the protein size of Scm3 varies drastically from 200 amino acids in S. cerevisiae to 1300 amino acids in $N$. crassa. Much of this size variation is due to addition of C-terminal nucleic acid binding domains (zinc-finger, metal chelating clusters, etc.) (Aravind et al., 2007). In humans and fission yeast, HJURP ${ }^{H . s .} / \mathrm{Scm}^{\text {S.p. }}$ requires Mis 18 to be recruited to the regional centromeres. In S. cerevisiae, there are no Mis 18 homologs so Scm3 achieves centromere targeting by binding to Ndc10, a protein which directly recognizes the CDEII sequence at the budding 
yeast centromere (Camahort et al., 2007; Espelin et al., 1997). Scm3 also binds to ATrich DNA so this may be part of the recruitment mechanism for its centromere targeting or nucleosome assembly activity (Xiao et al., 2011). Mis18 and Scm3 physically interact in fission yeast, and recently, it has been shown that HJURP binds to Mis $18 \alpha$ and Mis18 $\beta$ (Nardi, et al. submitted 2014, (Wang et al., 2014). This physical interaction between Mis18 and $\mathrm{HJURP} / \mathrm{Scm} 3$ is likely to contribute to HJURP centromere recruitment, though other factors and interactions may be involved.

HJURP is also required for CENP-A loading in Xenopus egg extracts, and human HJURP can substitute for Xenopus HJURP despite having little homology (Bernad et al., 2011). An HJURP homolog, CAL1, has recently been identified in Drosophila where it is required for CENP-A loading during mitosis (Chen et al., 2014; Erhardt et al., 2008; Mellone et al., 2011). Studies in nematodes have not identified an HJURP homolog, but the fact that yeast, fish, flies (CAL1), Xenopus, and humans all have these proteins does suggest the protein family is old but may have been lost in some multi-cellular eukaryotes.

The crystal structure of full length HJURP has not been solved, but it does have several identifiable domain features. The most well conserved region of the HJURP/Scm 3 proteins is contained in the first $80 \mathrm{~N}$-terminal amino acids. This region, the $\mathrm{Scm} 3$ domain, is the region of the protein that interacts with CENP-A in yeast and humans (Barnhart et al., 2011; Bassett et al., 2012b; Mizuguchi et al., 2007; Shuaib et al., 2010). Human HJURP also contains a conserved domain spanning amino acids 228-304 that is common to other vertebrates including $X$. tropicalis and G. gallus. HJURP also contains two repeated regions, which are only repeated in humans (Aravind et al., 2007; 
Sanchez-Pulido et al., 2009). Chapter III of this work will characterize the vertebrate conserved domain of HJURP as an RNA binding domain that is required for efficient CENP-A deposition. The two conserved, C-terminal repeated regions within HJURP are required for HJURP centromere targeting and HJURP homo-dimerization. The first

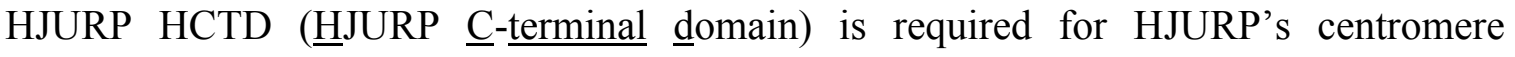
targeting. The second HCTD within HJURP is a self-dimerization domain, and this HJURP dimerization is required for efficient CENP-A loading in human cells (Zasadzinska et al., 2013).

Three studies solved crystal structures of HJURP/Scm3 binding to the CENPA/Cse4 - H4 heterodimer using budding yeast (S. cerevisiae and K. lactis) and human proteins (Cho and Harrison, 2011; Hu et al., 2011; Zhou et al., 2011). In all cases, HJURP $^{\text {aal } 1-80} / \mathrm{Scm} 3$ forms an N-terminal $\alpha$-helix followed by a 3 -stranded $\beta$-sheet. This N-terminal $\alpha$-helix packs against $\alpha-2$ helix of the histone fold domain of CENP-A/Cse4 (within the CATD). HJURP/Scm3 binding along the $\alpha-2$ helix of CENP-A/Cse4 precludes the CENP-A/Cse4 self-dimerization seen in the CENP-A/H4 heterotetramer structure (Sekulic et al., 2010). The $\beta$-sheet of HJURP/Scm3 interacts along a positively charged groove of the CENP-A/Cse4 molecule where DNA will wrap once a nucleosome is assembled. Thus, as a true chaperone, HJURP/Scm3 binding precludes CENP-A/Cse4 from spuriously interacting with DNA (Cho and Harrison, 2011; Hu et al., 2011; Zhou et al., 2011). These studies indicate HJURP/Scm3, CENP-A/Cse4, and H4 exist as a heterotrimer in the pre-nucleosomal form. In humans, self-dimerization of HJURP is thought to allow two heterotrimeric HJURP-CENP-A-H4 complexes to localize to 
centromeres concurrently, thereby enabling octameric nucleosome assembly (Zasadzinska et al., 2013).

In addition to chaperoning CENP-A/Cse4 to the centromere, HJURP/Scm3 also acts as a CENP-A specific nucleosome assembly factor. In budding yeast, Scm3 was demonstrated to specifically assemble Cse4 nucleosomes over $\mathrm{H} 3$ nucleosomes in vitro (Camahort et al., 2009; Mizuguchi et al., 2007; Shivaraju et al., 2011). Human HJURP aa1208 can also stably assemble CENP-A nucleosomes both in vivo and in vitro and this work will be discussed in chapter II (Barnhart et al., 2011). Similarly, in Drosophila cells, the CID chaperone CAL1 is sufficient to assemble CID-containing nucleosomes at a noncentromeric LacO array (Chen et al., 2014). Lastly, RbAP48, which co-purifies with CID from Drosophila cells, can assemble CID/H4/H2A/H2B nucleosome particles in vitro when used in a plasmid supercoiling assay (Shivaraju et al., 2011). The role of RbAP46/48 is relatively unexplored at the centromere, but its association with Mis18, HJURP, and CENP-A across multiple species makes it an interesting candidate for further study.

In addition to acting as the CENP-A/Cse4 specific chaperone, HJURP/Scm3 is also sufficient to assemble centromeric nucleosomes. It is currently unknown if the Mis 18 complex plays any active role in CENP-A deposition or if it instead acts only as the recruiting complex for HJURP/Scm3. Additionally, Npm1, which associates with HJURP in the prenucleosomal complex as mentioned, has CENP-A nucleosome assembly activity in vitro but whether it plays a role in CENP-A nucleosome formation in vivo is also not clear (Barnhart et al., 2011; Dunleavy et al., 2009). A snapshot of CENPA loading at human centromeres in early G1 is depicted in Figure 1-4 to summarize the 
important players discussed so far. The fate of this newly deposited CENP-A will be discussed in the next section. 


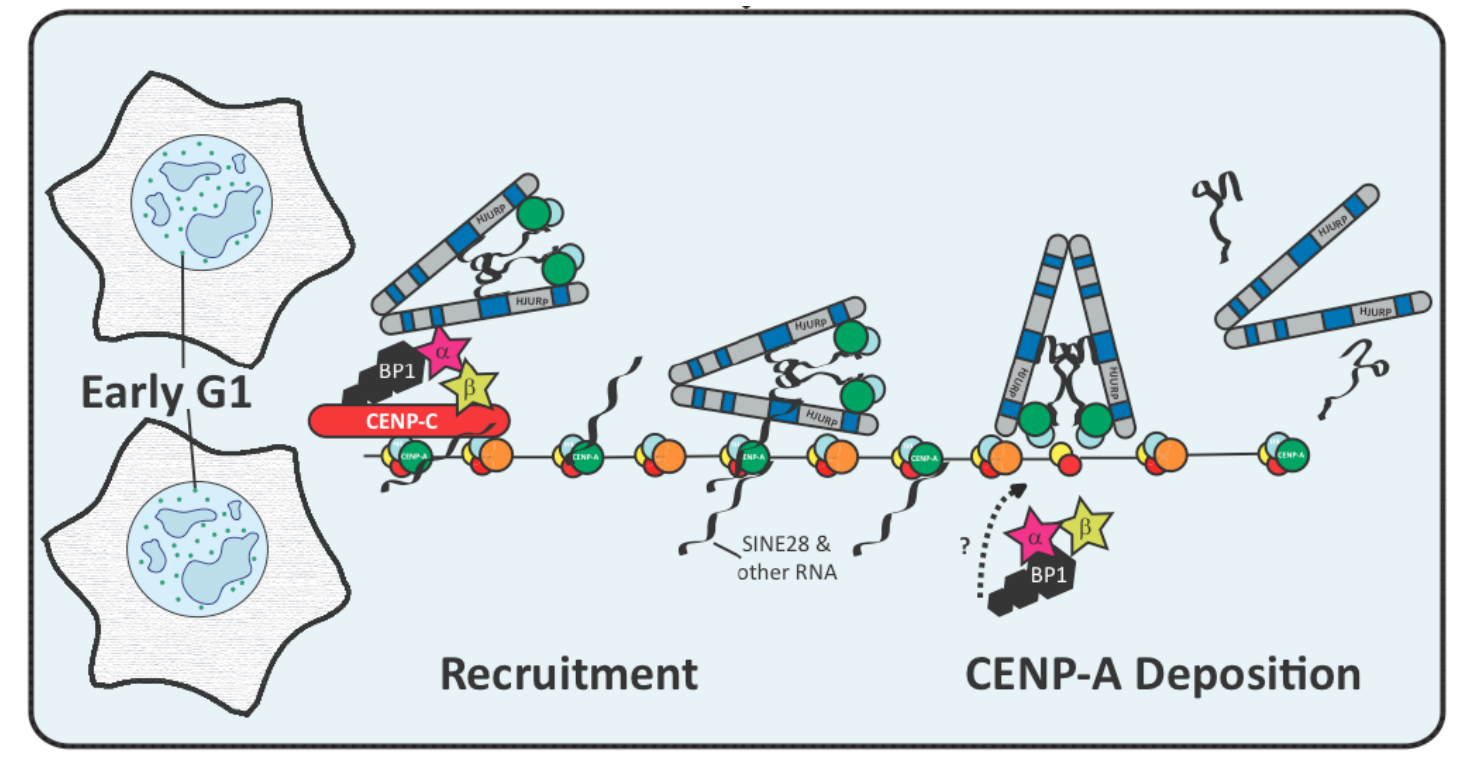

Figure 1-4: Schematic of CENP-A loading in early G1 in human cells.

In late anaphase, the Mis18 complex (Mis18 $\alpha$, Mis18 $\beta$, Mis18BP1) localizes to centromeres. Both Mis18BP1 and Mis18 $\beta$ interaction with CENP-C are both required for full Mis18BP1 recruitment. Dimerized HJURP bound to pre-nucleosomal CENP-A/H4 heterodimers is recruited to centromeric chromatin by the Mis18 complex. RNA may play a role in stabilizing HJURP's recruitment to centromeres and will be discussed in a coming section. HJURP deposits CENP-A at centromeric chromatin. Whether the Mis18 complex is actively involved in the CENP-A deposition process is not known. HJURP and the Mis18 complex leave the centromere and the cell progress into G1 with its full complement of CENP-A. 
Players in CENP-A Chromatin Maturation and Stability

\section{CENP-A Maturation and Stability}

In addition to the CENP-A assembly factor HJURP, the CCAN proteins CENP-C, CENP-N, CENP's-H/I/K, and the Mis18 priming complex, a handful of other proteins have been shown to affect CENP-A loading at centromeres in human cells. Nucleosome remodeler and spacing protein Rsf-1 associates with CENP-A nucleosomes and localizes to centromeres during mid-G1. Depleting cells of Rsf-1 results in misaligned kinetochores and loss of stably incorporated CENP-A nucleosomes by high salt washing (Perpelescu et al., 2009). Additionally, the FACT complex and chromatin remodeling factor CHD1 also localizes to centromeres, but in a CENP-H complex-dependent manner. Depletion of the FACT subunit SSRP1 results in less efficient CENP-A loading at centromeres while it has no effect on H3 loading (Okada et al., 2009). Lastly, MgcRacGap, the GTPase Cdc42, and its GEF ECT2 localize to centromeres in late G1. They are proposed to "mature" newly deposited CENP-A nucleosomes by either adding or removing an epigenetic mark before the cell enters S-phase, however, the exact mechanism was not identified in the paper. It is clear that inhibiting MgcRacGAP causes loss of new CENP-A at centromeres (Lagana et al., 2010). If true, these findings present an interesting model where newly deposited CENP-A must be matured by these factors in order to be stably incorporated, otherwise, it is recognized as erroneously deposited and is removed (Figure 1-3).

CENP-A deposition also requires the condensin II complex in vertebrate cells. Knocking down a non-SMC subunit of the condensin II complex resulted in loss of 
CENP-A deposition in Xenopus extracts (Bernad et al., 2011). Additionally, depleting the SMC subunits, which are common to both condensin I and condensin II, causes a reduction of CENP-A intensity in human cells (Samoshkin et al., 2009). Chapter IV of this work will discuss an interaction between the condensin II complex and HJURP as well as a role for specifically condensin II in CENP-A deposition in human cells.

Many of these proteins needed for stable CENP-A deposition localize to centromeres later in the cell cycle after CENP-A is initially loaded in early G1 and are thought to remodel and/or space CENP-A nucleosomes. Because of this, it is possible CENP-A nucleosomes are not completely stable when they are initially deposited and that a further remodeling and maturation step is necessary to complete the deposition process (Figure 1-3).

\section{Removal of Non-Centromeric CENP-A}

In addition to the specific targeting of the CENP-A deposition machinery and the CCAN uniquely marking centromeric chromatin, organisms employ an additional level of regulation to make sure CENP-A nucleosomes are only deposited at centromeric chromatin. When over-expressed, CENP-A/Cse4 can lose its centromere-specific localization and become mis-incorporated at other chromatin regions in yeast, flies, and humans (Collins et al., 2007; Heun et al., 2006; Moreno-Moreno et al., 2006; Van Hooser et al., 2001). This mis-localized CENP-A/Cse4 must then be cleared in order to avoid chromosome segregation mistakes and neocentromere formation. A balance of tightly controlled expression and proteolysis is thought to be the main mechanism by which CENP-A is kept specifically at the centromere. In yeast and flies, an E3 ubiquitin ligase mediates Cse4/CID degradation when it is over-expressed. Psh1 in yeast and Ppa in flies, 
these proteins can reverse the effects of $\mathrm{Cse} 4 / \mathrm{CID}$ overexpression by ubiquitinating the mis-localized proteins and targeting them for degradation by the proteasome (Hewawasam et al., 2010; Moreno-Moreno et al., 2011; Ranjitkar et al., 2010). The E3 ligase that fulfills this role in human cells has not been identified. Though, in human cells, over-expressed CENP-A was recently shown to use a distinct chaperone, DAXX, instead of its G1-specific chaperone HJURP for its incorporation following overexpression (Lacoste et al., 2014). This and data showing the histone/chaperone expression levels are tightly linked in cells suggests proper chaperone binding of CENPA by HJURP also protects it from mis-incorporation and subsequent degradation (Dunleavy et al., 2009; Foltz et al., 2009). Supporting the importance of tightly regulated CENP-A and chaperone expression, CENP-A and HJURP over-expression are commonly found in cancers (Kato et al., 2007; Tomonaga et al., 2003). 


\section{RNA, Transcription and the Centromere}

RNA is a component feature at plant and animal centromeres. There is a growing amount of evidence that transcription at the centromere in human cells is required for CENP-A deposition (Bergmann et al., 2011; Chan et al., 2012). RNA may also fulfill a structural role at the centromere as the resulting RNA from this transcription plays a major role in centromere function and kinetochore assembly (Chan and Wong, 2012; Du et al., 2010; Ferri et al., 2009; Ohkuni and Kitagawa, 2011; Wong et al., 2007). RNA and centromeric transcription are important for many centromere processes, and these are summarized in Figure 1-5.

The most well established relationship between the centromere and RNA is the creation and maintenance of pericentromeric heterochromatin in fission yeast. Continued transcription of small RNA's then RNAi-mediated processing of these transcripts from centromere-flanking repeats is required for the establishment of pericentromeric heterochromatin, and this flanking heterochromatin is also required for de novo CENPA/Cnp1 assembly in the central centromere core (Folco et al., 2008; Volpe et al., 2002). This mechanism is not strictly conserved in higher eukaryotes. There is evidence that HP1 recruitment is reduced when long pericentromeric RNA's accumulate in response to Dicer depletion in hybrid chicken cells harboring a human chromosome 21, but no effect on CENP-A stability was observed (Fukagawa et al., 2004). However, eliminating RNA from pericentromeric repeats also causes HP1 localization loss, thus some balance of the siRNA's and the long non-coding RNA's is necessary for proper HP1 recruitment (Maison et al., 2002; Muchardt et al., 2002). 
Another important aspect of the centromere/RNA relationship is the effect of RNA on centromere function and CENP-A chromatin establishment. ChIP studies from maize, mice, and humans found association of CENP-A/CENH3 with alpha-satellite RNA (Ferri et al., 2009; Topp et al., 2004; Wong et al., 2007). In budding yeast, transcription factor binding at centromeres is required for centromere function. Ectopically driving RNA Pol II activity over the CEN region is sufficient to rescue the high chromosome missegregation rates induced by transcription factor binding loss (Ohkuni and Kitagawa, 2011). Studies in human cells found that RNA Pol II inhibition during mitosis induces a reduction in the number of alpha-satellite transcripts detected at the centromere as well as slight effects on lagging chromosomes during anaphase (Chan et al., 2012). This indicates some level of transcription is required for proper centromere and kinetochore function in human cells during mitosis. Additionally, transcriptionally active LINE retrotransposons within CENP-A regions on a human neocentromere are required for successful CENP-A binding and incorporation (Chueh et al., 2009). Studies in marsupials have also identified a novel class of small RNA that is composed of satellite and retroviral sequences and emanates from the centromeres in these cells (Carone et al., 2009). Artificially inhibiting transcription by targeting LSD1 to a human artificial chromosome centromere caused a loss of HJURP recruitment and subsequent CENP-A loss (Bergmann et al., 2011). Complementary to this, driving high transcription over a HAC centromere also caused kinetochore inactivation and CENP-A loss (Bergmann et al., 2012). These studies emphasize that the amount of transcription is the key factor for centromere stability. 
One reason transcription at centromeres is required for accurate chromosome segregation may be due to an RNA requirement for proper CCAN and kinetochore recruitment. In support of this, previous studies have indicated that the CPC members Aurora B, Survivin, and INCENP interact with RNA and require RNA for their centromeric localization (Ferri et al., 2009; Wong et al., 2007). Similarly, CENP-C requires RNA for its centromeric recruitment and for its DNA binding activity (Chan et al., 2012; Du et al., 2010; Wong et al., 2007). Treating mammalian cells with RNase results in a reduction of CENP-C, CENP-W, and even CENP-A at centromeres (Carone et al., 2009; Chun et al., 2011; Wong et al., 2007). Lastly, in chapter III of this work, we will show data demonstrating an RNA dependency in HJURP and Mis18 $\alpha$ recruitment.

Lastly, nucleoli and centromeres have a documented relationship in higher eukaryotes. Nucleoli are well known for being the sites of ribosomal RNA synthesis and as such, are concentrated with RNA. In G1, S, and G2 nuclei, centromeres cluster around nucleoli on average $60 \%$ of the time (Foltz et al., 2009). This is not a well-understood phenomenon but may play a role in centromere assembly and maintenance. There is recent evidence in Drosophila that centromere nucleolar clustering mediates centromere pairing in meiosis I (Unhavaithaya and Orr-Weaver, 2013). Many of the proteins that require RNA for their centromeric recruitment, also localize to nucleoli. HJURP, CENPC, CENP-W, INCENP, Npm1, and the Mis18 complex all enrich at nucleoli in human cells (Chun et al., 2011; Dunleavy et al., 2009; Ferri et al., 2009; Foltz et al., 2009; Wong et al., 2007). CENP-W interacts with Npm1, and this interaction is RNase A sensitive (Chun et al., 2011). All these data suggest nucleoli may play a role in centromere and 
kinetochore assembly and support the notion that RNA serves an important functional role in these processes.

Chapter III of this work will investigate an RNA binding region of the CENP-A chaperone HJURP. We will show that this region of HJURP is required for its nucleolar localization and for efficient CENP-A deposition. We additionally show the specificity of this region for binding a recently identified SINE28 small RNA that associates with the human centromere throughout the cell cycle. 


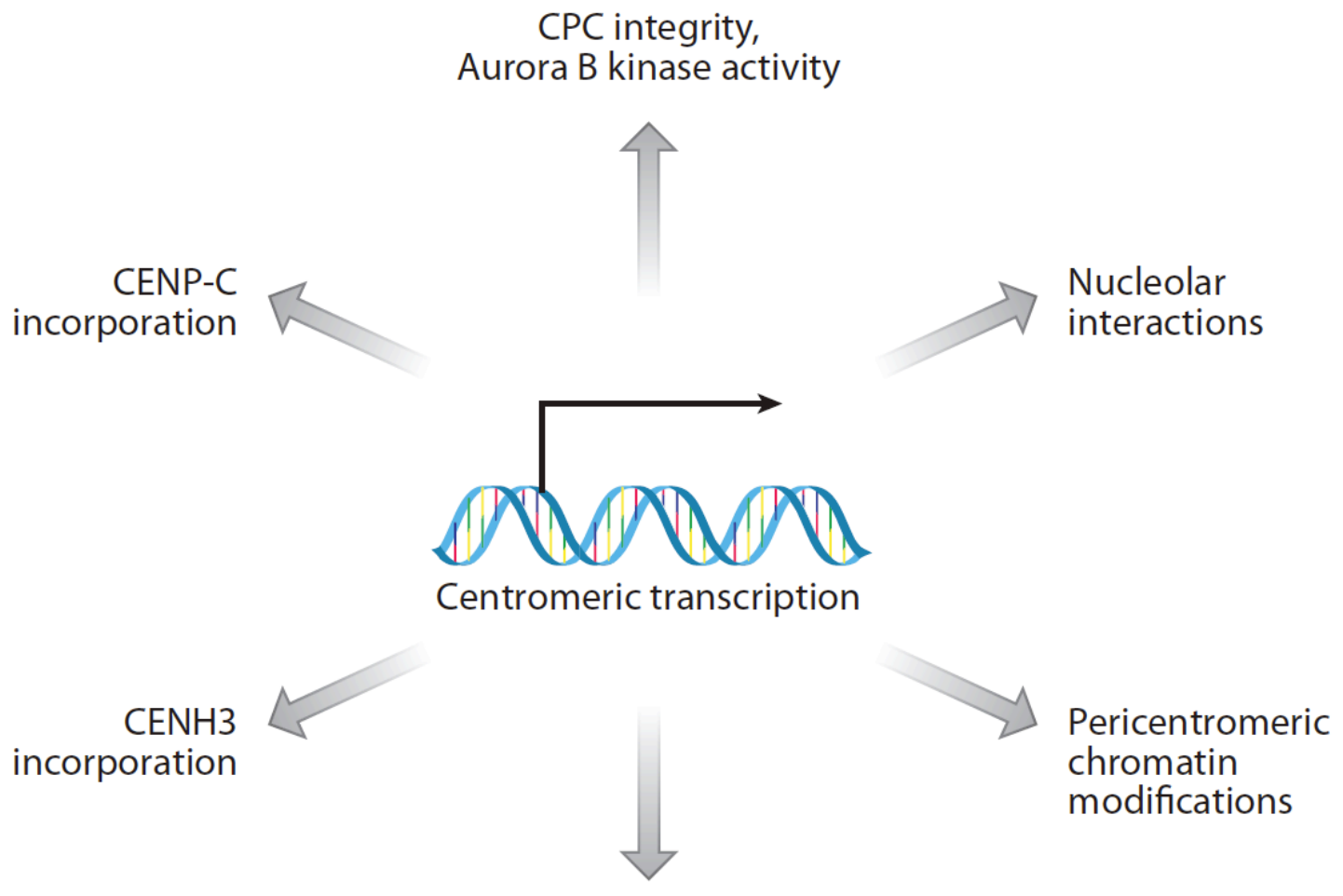

Histone $\mathrm{H} 3$ modifications

Figure 1-5. Diagram of centromeric events that are influenced by centromeric transcription or RNA itself.

The physical presence of RNA at the centromere and centromeric transcription both play important roles in centromere biology. RNA plays a structural role in the stability of various CCAN members, CPC members, and some of the CENP-A deposition machinery at the centromere (Chapter III). A moderate amount of centromeric transcription is also required for stable CENP-A deposition. Transcription of pericentromeric repeats is critical for their formation and maintenance in budding yeast. Centromeres interact with nucleoli and many centromere proteins enrich in nucleoli.

Figure included with permission, (Gent and Dawe, 2012) 


\section{CHAPTER II: HJURP IS A CENP-A CHROMATIN ASSEMBLY $\underline{\text { FACTOR SUFFICIENT TO FORM A FUNCTIONAL DE NOVO }}$ KINETOCHORE}

This chapter is based on the following publication:

Barnhart, MC., Kuich, PHJL., Stellfox, ME., Ward, JA., Bassett, EA., Black, BE., and Foltz, DR. "HJURP is a CENP-A chromatin assembly factor sufficient to form a functional de novo kinetochore.” Journal of Cell Biology (2011), 194(2):229-243. 


\section{$\underline{\text { Abstract }}$}

Centromeres of higher eukaryotes are epigenetically marked by the centromerespecific CENP-A nucleosome. New CENP-A recruitment requires the CENP-A histone chaperone HJURP. Here, we show that a Lac repressor fusion of HJURP drove the stable recruitment of CENP-A to the LacO array at a non-centromeric locus. Ectopicallytargeted CENP-A chromatin at the LacO array was sufficient to direct the assembly of a functional kinetochore as indicated by the recruitment of the constitutive centromereassociated network proteins (CCAN), the microtubule-binding protein NDC80, the spindle assembly checkpoint proteins Mad2 and Aurora B, and the formation of stable kinetochore-microtubule attachments. A carboxyl-terminal fragment of HJURP was able to assemble CENP-A nucleosomes in vitro, demonstrating that HJURP harbors nucleosome assembly activity. Furthermore, HJURP recruitment to endogenous centromeres required the Mis18 complex. Together, this work suggest that one role of the Mis 18 complex in CENP-A deposition is to recruit HJURP to centromeres and that the CENP-A nucleosome-assembly activity of HJURP is responsible for centromeric chromatin assembly to maintain the epigenetic mark during each cell cycle. 


\section{$\underline{\text { Introduction }}$}

Centromeric chromatin is defined by the incorporation of a unique nucleosome

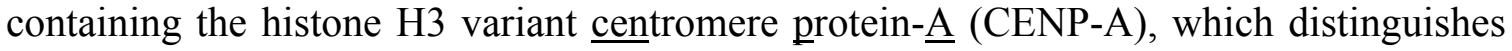
this locus from general chromatin. Centromeric chromatin plays an integral role in organizing and controlling chromosome segregation. The centromere is the site of microtubule attachment and checkpoint signaling during mitosis and also organizes the constitutive centromere components throughout the cell cycle (Cheeseman et al., 2008; Cleveland et al., 2003; Musacchio and Salmon, 2007).

The human centromere contains hundreds of thousands to millions of base-pairs of DNA arranged in alpha-satellite higher order repeats (Allshire and Karpen, 2008; Cleveland et al., 2003; Schueler and Sullivan, 2006). Despite the characteristic alphasatellite DNA content of centromeres, the existence of stable dicentric chromosomes where a single centromeric region remains "active", and the formation of neocentromeres at non-alpha-satellite repeat regions has led to the understanding that centromere specification is an epigenetic process dictated by the presence of CENP-A nucleosomes (Burrack and Berman, 2012; Marshall et al., 2008). The mechanism by which CENP-A is assembled at pre-existing centromeric sites, whether at canonical centromeres or neocentromeres, remains unclear.

The presence of the CENP-A nucleosome within centromeric chromatin directs the recruitment of a large set of proteins present at the centromere throughout the cell cycle (Musacchio and Salmon, 2007). These proteins can be divided into partially overlapping sub-complexes including the CENP-A ${ }^{\mathrm{NAC}}$ (nucleosome associated complex), 
CENP-A $^{\text {CAD }}$ (CENP-A distal components), and CENP-H/I complexes, and are collectively termed the constitutive centromere associated network (CCAN) (Foltz et al., 2006; Obuse et al., 2004; Okada et al., 2009). CENP-N and CENP-C, within the CENP$\mathrm{A}^{\mathrm{NAC}}$, are able to independently discern centromeric CENP-A containing chromatin from general chromatin by directly recognizing the CENP-A nucleosome (Carroll et al., 2010; Carroll et al., 2009; Guse et al., 2011).

Several models have been proposed to explain how CENP-A nucleosomes may be differentiated from H3.1 nucleosomes. Data from budding yeast and humans predict an octameric CENP-A containing nucleosome with a more compact structure relative to the histone H3.1 nucleosome (Black et al., 2007a; Black et al., 2007b; Camahort et al., 2009; Hasson et al., 2013; Miell et al., 2013; Sekulic et al., 2010; Tachiwana et al., 2011). Also proposed are changes in the CENP-A nucleosome composition that include single copies of each of the four core histones (heterotetramer) in flies and humans (Dalal et al., 2007; Dimitriadis et al., 2010). Others find a hexameric form in budding yeast that lacks histones $\mathrm{H} 2 \mathrm{~A}$ and $\mathrm{H} 2 \mathrm{~B}$ but includes the non-histone CENP-A $\mathrm{Cse}^{\mathrm{Cs}}$ chaperone Scm3 or a lack of histones $\mathrm{H} 2 \mathrm{~A}$ and $\mathrm{H} 2 \mathrm{~B}$ at centromeres in fission yeast suggesting an alternate form (Mizuguchi et al., 2007; Williams et al., 2009). In humans, the crystal structure of the CENP-A nucleosome reveals an octameric arrangement with two copies of each core histone (Tachiwana et al., 2011). Further evidence supporting an octameric CENP-A nucleosome in flies and humans comes from data demonstrating the dimerization interface within the CENP-A histone is required for stable deposition (Bassett et al., 2012a; Zhang et al., 2012). Finally, it has been suggested that centromeric nucleosomes wrap DNA in a right-handed path around the CENP-A/CenH3 containing histone core, in 
contrast to the left handed wrapping of the canonical H3 nucleosome (Furuyama and Henikoff, 2009). This has been refuted in humans with a crystal structure of octameric CENP-A nucleosomes wrapping DNA in a left-handed manner (Tachiwana et al., 2011). Taken together, the multitude of data suggest that CENP-A may exist in a variety of forms between species, but it is accepted that octameric CENP-A nucleosomes exist in abundance at human centromeres and are important for stable CENP-A nucleosome assembly. All of these structural distinctions may contribute specificity to the selective assembly of CENP-A nucleosomes into centromeric loci or to the recruitment of a unique set of proteins to the centromeric chromatin.

The re-distribution of pre-existing CENP-A nucleosomes between newly synthesized sister DNA strands during S-phase necessitates the incorporation of new CENP-A nucleosomes during each round of cell division in order to maintain centromeric identity. Distinct histone chaperones for the different histone $\mathrm{H} 3$ variants function to couple the deposition of the appropriate histone variant to a unique site within the genome at distinct times during the cell cycle (Ransom et al., 2010). In the case of vertebrates, new CENP-A is recruited to centromeres in a pre-nucleosomal complex with HJURP during early G1 (Bernad et al., 2011; Dunleavy et al., 2009; Foltz et al., 2009; Jansen et al., 2007; Shuaib et al., 2010). Point centromeres of S. cerevisiae and regional centromeres of $S$. pombe also require the HJURP homolog, $\mathrm{Scm} 3$, for the recruitment of their respective CENP-A homologs (Camahort et al., 2007; Mizuguchi et al., 2007; Pidoux et al., 2009; Williams et al., 2009). The interaction between HJURP and CENP-A depends on the centromere targeting domain (CATD) of CENP-A (Black et al., 2004; Foltz et al., 2009). The CATD is a portion of the histone fold domain of CENP-A that 
confers structural changes that alter the shape, surface, and conformational flexibility of the complexes into which CENP-A assembles, relative to its conventional counterpart $\mathrm{H} 3$ (Black et al., 2007a; Sekulic et al., 2010; Shelby et al., 1997) This same region dictates the interaction of CENP-A with the Scm3 domain of HJURP, as well as CENP-A's association with the constitutive centromere protein CENP-N (Carroll et al., 2009; Foltz et al., 2009), though in frogs, CENP-N binds the C-terminal tail of CENP-A not the CATD (Guse et al., 2011). These data suggest that the structural differences imparted by the CATD mediate both the correct localization and incorporation of CENP-A within the genome and the subsequent recruitment of the appropriate CCAN proteins required for building the centromere.

New CENP-A nucleosome assembly also requires the human Mis18 complex

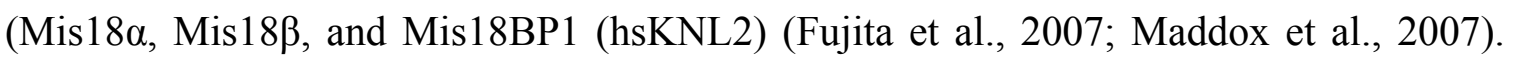
This complex is initially recruited to centromeres during telophase and remains associated with CENP-A containing chromatin into G1. The Schizosaccharomyces pombe homolog of Mis18 is required for the localization of Scm3 and CENP-A to centromeres (Camahort et al., 2007; Mizuguchi et al., 2007; Pidoux et al., 2009; Stoler et al., 2007; Williams et al., 2009). In vertebrates, Mis18 is not found as part of the CENP-A/HJURP prenucleosomal complex, nor is it associated with isolated CENP-A nucleosomes, but it is contained within CENP-A containing chromatin (Foltz et al., 2009; Foltz et al., 2006; Maddox et al., 2007). Recent data from our lab indicates Mis18 $\alpha$ and Mis18 $\beta$ can both bind to CENP-A in vitro (Nardi et al., unpublished results). Mis $18 \beta$ was also recently shown to bind to the CENP-A chaperone HJURP (Wang et al., 2014). Because it localizes to centromeres just prior to new CENP-A loading, it is hypothesized that Mis18 
primes the centromere for CENP-A deposition through a mechanism that may involve histone acetylation (Fujita et al., 2007; Hayashi et al., 2004). Another possibility is Mis18 acts as a licensing factor that dictates a single CENP-A assembly event per single Mis18 molecule (Nardi et al. unpublished data).

Here, we investigate if CENP-A chromatin can be stably established at a noncentromeric location in the genome, specifically asking if HJURP is sufficient to establish this CENP-A chromatin in vivo. We demonstrate recruitment of HJURP to a non-centromeric LacO array is sufficient to drive the stable association of CENP-A at the array and to recreate a functional centromere. The HJURP-deposited CENP-A at the array is competent to recruit CCAN proteins, the kinetochore protein NDC80, the spindle assembly checkpoint proteins Aurora B and $\mathrm{Mad}$, and form stable microtubule attachments during mitosis. These attachments lead to chromosome breakage of the de novo dicentric chromosome when the cell enters anaphase. The same fragment of HJURP that contains the Scm3 domain is able to specifically assemble CENP-A nucleosomes in vitro. We additionally show the Mis18 complex is required for HJURP recruitment to endogenous centromeres. However, when this recruitment step is bypassed by tethering HJURP to the LacO array, CENP-A deposition can still occur in the absence of Mis18 at this non-centromeric location. Together, these data establish the sufficiency of HJURP in the recruitment of CENP-A and in the assembly of CENP-A into stable nucleosomes both in vivo and in vitro. Additionally, they delineate the roles of Mis 18 and HJURP in CENPA recruitment and assembly. 


\section{$\underline{\text { Results }}$}

\section{HJURP recruitment is sufficient to drive CENP-A deposition into chromatin}

HJURP is required for CENP-A deposition and is localized to centromeres during early G1 when new CENP-A is incorporated into centromeres (Dunleavy et al., 2009; Foltz et al., 2009; Jansen et al., 2007). We hypothesized that recruitment of HJURP may couple CENP-A delivery and nucleosome assembly at centromeres by chaperoning CENP-A to centromeres and providing intrinsic CENP-A deposition activity. If true, HJURP recruitment to a non-centromeric site should be sufficient to dictate the site of CENP-A nucleosome deposition. We ectopically localized HJURP to a non-centromeric site within the genome by expressing a LacI-HJURP fusion protein in U2OS cells containing 200 copies of a $256 \times \mathrm{LacO} / 96 \mathrm{xTRE}$ array on chromosome 1 (Janicki et al., 2004). We then determined if CENP-A deposition occurred at this site.

LacO/TRE U2OS cells were transiently transfected with either mCherry-LacI or the mCherry-LacI-HJURP fusion (hereafter called LacI or LacI-HJURP). GFP-TetR was co-transfected to independently determine the location of the array. The LacI-HJURP and GFP-TetR fusion proteins successfully localized to and marked the LacO/TRE array (Figure 2-1 A). When HJURP was localized at the array, endogenous CENP-A was also enriched in $61.3 \%$ of transfected cells, 48 hours after transfection (Figure 2-1 A, C). This was in contrast to $1 \%$ of cells showing CENP-A co-localization with the array when the LacI control was expressed, demonstrating the LacO/TRE array did not consistently overlap with an endogenous centromere (Figure 2-1 A, C). We also noted that the number of CENP-A positive LacI-HJURP arrays increased with time where by 72 hours 
following transfection, $92 \%$ of arrays were positive for endogenous CENP-A. This suggests cell cycle position and/or chromatin alteration at the LacO array may contribute to when and if CENP-A can be deposited at the array (Suppl. Figure 2-2 A).

We disrupted the LacI interaction with the LacO-array using IPTG to determine if CENP-A was stably associated with the underlying chromatin or whether it was tethered at the array solely through its interaction with LacI-HJURP. We expected that the portion of the CENP-A tethered at the array through its binding to LacI-HJURP would be removed by treatment with IPTG. Any remaining CENP-A signal at the array following IPTG treatment would indicate stable association of CENP-A with the DNA. Cells were treated with $10 \mathrm{mM}$ IPTG for 1 hour prior to fixation, 48 hours after transfection. Under these conditions, LacI-HJURP was no longer visible at the array in cells counted for CENP-A stability (array marked by GFP-TetR) (Figure 2-1 B). CENP-A persisted at the array in 51.6\% of IPTG-treated cells where LacI-HJURP was no longer visible (Figure 2$1 \mathrm{C})$. We observed a $46 \%$ decrease in the average intensity of the arrays in cells treated with IPTG consistent with a subset of CENP-A being tethered at the array through binding to HJURP (Figure 2-1 D). The stable association of CENP-A with the LacO DNA following removal of HJURP is consistent with the assembly of CENP-A containing chromatin at the array (Figure 2-1 B, C). Alternatively, the CENP-A observed following removal of HJURP may persist as a prenucleosomal form pending its assembly by other factors similarly to what has been proposed previously in RSF1 knockdowns (Perpelescu et al., 2009).

We used an NIH-3T3 cell line containing a LacO-SceI-TetO array to demonstrate that the stable recruitment of CENP-A by LacI-HJURP was not a unique property of the 
U2OS cells or the genomic location of the array (Soutoglou et al., 2007). Transfected YFP-CENP-A was recruited to the LacO array when LacI-HJURP was expressed and remained stably associated with the array when the cells were treated with IPTG, similarly to what we observed in the U2OS cell line, even out to 24 hours in some examples (Suppl. Figure 2-1 A-C).

To determine if the stably assembled, non-centromeric CENP-A was sufficient to act as an epigenetic mark to then recruit more CENP-A, U2OS-LacO cells were transfected with LacI-HJURP for 24 hours then treated with IPTG to remove the LacIHJURP after it had assembled CENP-A at the LacO array. 8 hours into the IPTG treatment, the cells were re-transfected with HA-CENP-A then allowed to grow for another 48 hours in the presence of IPTG. Cells were then fixed and stained with an antibody to HA. If HA-CENP-A was present at the array, this indicated the initial endogenous CENP-A assembled by LacI-HJURP (prior to its removal with IPTG) was able to act as an epigenetic mark signaling that more CENP-A should be recruited to and deposited at the non-centromeric site. We observed a low percentage (6\%) of GFP-TetR positive arrays that were also positive for the HA-CENP-A indicating epigenetic recruitment by CENP-A (Suppl. Figure 2-2 B).

The extent of homology between the yeast Scm3 proteins and HJURP is contained within a small 52 amino acid stretch in the amino terminus of HJURP (Sanchez-Pulido et al., 2009). A fragment of HJURP that contains the Scm3 homology domain is sufficient to mediate the interaction of CENP-A and HJURP but is not able to direct HJURP to centromeres (Shuaib et al., 2010; Zasadzinska et al., 2013) (Suppl. Figure S2-3 A-C). It is reasonable to suppose that HJURP and Scm3 mediate similar 
functions with respect to CENP-A nucleosome deposition; therefore, we tested if the region of HJURP that includes the Scm3 homology domain (HJURP ${ }^{\mathrm{Scm} 3}$, amino acids 1208), which can interact with CENP-A but is unable to localize to centromeres, is sufficient to direct the stable association of CENP-A at the array. HJURP ${ }^{\mathrm{Scm} 3}$ was fused to mCherry-LacI (called LacI-HJURP ${ }^{\mathrm{Scm} 3}$ ) and transfected into the LacO containing U2OS cells. Targeting LacI-HJURP ${ }^{\mathrm{Scm} 3}$ resulted in the recruitment of endogenous CENPA to the array in $39.9 \%$ of cells after 48 hours in culture, similar to the full-length protein (Figure 2-1 A, C). We removed LacI-HJURP ${ }^{\mathrm{Scm} 3}$ from the array using IPTG and endogenous CENP-A remained associated with the chromatin in $32.8 \%$ of cells, similarly to full-length HJURP (Figure 2-1 B, C). LacI-HJURP ${ }^{\mathrm{Scm} 3}$ recruited less CENP-A to the array (Figure 2-1 D); however, the proportion of CENP-A retained at the array in LacIHJURP $^{\mathrm{Scm} 3}$ transfected, IPTG treated cells was greater than full-length HJURP (Figure 21 D). Cells transfected with LacI-HJURP ${ }^{\text {Scm3 }}$ showed only a $5 \%$ decrease in average intensity in cells treated with IPTG relative to controls. Together, these data demonstrate HJURP and its N-terminal CENP-A binding region are sufficient to establish stable CENP-A chromatin at a non-centromeric location in the genome. 
Figure 2-1. HJURP-dependent CENP-A recruitment and incorporation into the LacO/TRE array.

(A) Recruitment of endogenous CENP-A to the LacO/TRE array in the presence of LacIHJURP or LacI-HJURP ${ }^{\mathrm{Scm} 3}$. All LacI constructs have an N-terminal mCherry tag. Representative images of pre-extracted cells treated with $0 \mathrm{mM}$ IPTG (A) or $10 \mathrm{mM}$ IPTG (B) for $1 \mathrm{hr}$ prior to fixation. Endogenous CENP-A was detected using a monoclonal anti-CENP-A antibody. mCherry-LacI fusions of HJURP or HJURP ${ }^{\mathrm{Scm} 3}$ and GFP-TetR marker are transiently transfected at equal ratios, and DNA is visualized using DAPI. Cells are fixed at $48 \mathrm{hrs}$ post-transfection. The scale bars $=5 \mu \mathrm{m}$. Arrows indicate the array. (C) Quantification of CENP-A staining at LacO/TRE array. Blue bars represent 0mM IPTG treatment and gray bars represent $10 \mathrm{mM}$ IPTG treatment. Error bars represent the standard deviation between two experiments. $\geq 30$ cells per condition, $n=2$. In the case of IPTG treatment, cells where residual mCherry signal was still visible at the array were excluded. (D) Quantification of the amount of CENP-A at the array in LacIHJURP and LacI-HJURP ${ }^{\mathrm{Scm} 3}$ transfected cells with and without treatment with IPTG. ( $>28$ cells/condition). Middle lines in each box represent the median integrated intensity for each condition and whiskers represent the max and min intensities observed. 

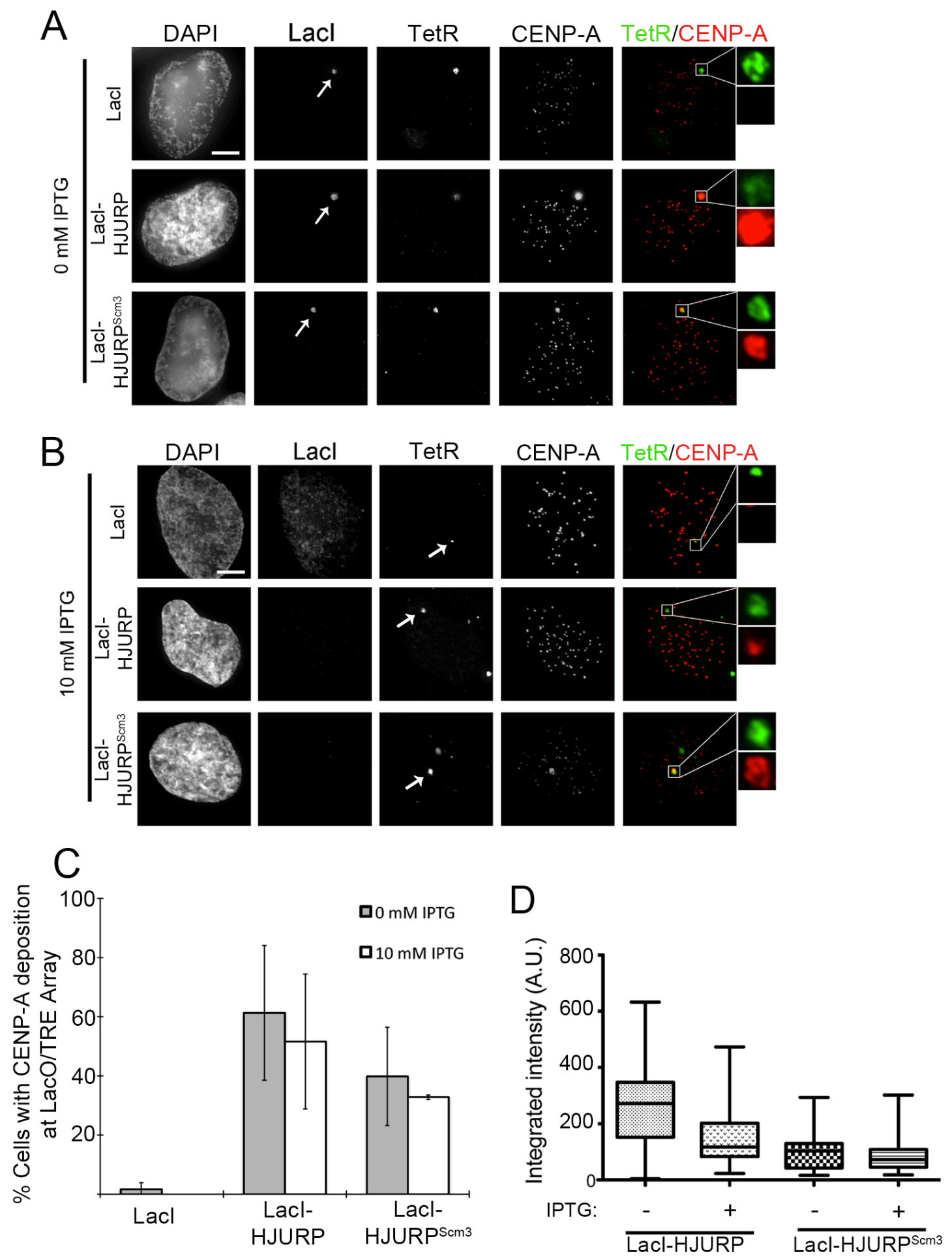
CENP-A deposition by HJURP recruits CENP-A nucleosome associated proteins

The recruitment of constitutive centromere proteins to human centromeres is thought to depend on the presence of CENP-A, as evidenced by affinity purifications, siRNA, and knockout experiments (Foltz et al., 2006; Liu et al., 2006; Obuse et al., 2004; Regnier et al., 2005). In vitro, CENP-N and CENP-C are able to directly and selectively recognize the CENP-A nucleosome (Carroll et al., 2010; Carroll et al., 2009). CENP-T co-purifies more closely with the $\mathrm{H} 3$ nucleosomes at the centromere and is a critical CCAN member for kinetochore function due to its direct interaction with the kinetochore protein Ndc80 (Gascoigne et al., 2011; Hori et al., 2008a; Nishino et al., 2013; Ribeiro et al., 2010). To test the ability of CENP-A nucleosomes to direct the recruitment of the constitutive centromere, we examined whether components of the CENP-A ${ }^{\mathrm{NAC}}$ are recruited to the LacO array after CENP-A was stably associated there using LacI-HJURP (as in Figure 2-1). LacO/TRE U2OS cells were co-transfected with LacI-HJURP and GFP-tagged constructs expressing CENP-C, CENP-N, CENP-M, or CENP-T, and fixed 72 hours later. Only cells with endogenous CENP-A present at the array and GFP-CENP signal at the centromeres were analyzed. We observed the recruitment of GFP-tagged CENP-C, CENP-N, CENP-M and CENP-T in $12 \%$ to $28 \%$ of co-transfected cells (Figure 2-2 A, C). CENP-N was most strongly recruited and selectively interacts with intact CENP-A nucleosomes but does not recognize the prenucleosomal heterotetramer, suggesting CENP-A assembled at the array may be nucleosomal (Carroll et al., 2009). CENP-T and CENP-C were also recruited to the CENP-A positive arrays. Given that these CCAN members are capable of interacting with the Ndc80 and Mis12 complexes, respectively, we next wanted to determine if kinetochore proteins were also recruited to 
the CENP-A positive LacO arrays (Gascoigne et al., 2011; Nishino et al., 2013; Przewloka et al., 2011; Screpanti et al., 2011). GFP signal was never observed at the array for any of the GFP-tagged CENP-A ${ }^{\mathrm{NAC}}$ proteins when cells were co-transfected with the LacI control construct (Figure 2-2 B).

LacI-HJURP ${ }^{\mathrm{Scm} 3}$ was sufficient to recruit and assemble CENP-A at the LacO arrays (Figure 2-1). The amount of CENP-A recruited by LacI-HJURP ${ }^{\mathrm{Scm} 3}$ was less than LacI-HJURP but what was recruited was more stably assembled (Figure 2-1 D). We therefore wanted to determine if different portions of HJURP were more or less efficient at recruiting CENP-A and subsequently the CCAN members, CENP-C and CENP-N (Suppl. Figure 2-4). Targeting LacI-HJURP ${ }^{\mathrm{Scm} 3}$ or LacI-HJURP then treating with IPTG resulted in the same CENP-A stability pattern previously observed (Figure 2-1 D, Suppl. Figure 2-4 E). Interestingly, less overall but more stable CENP-A recruitment mediated by LacI-HJURP ${ }^{\mathrm{Scm} 3}$ resulted in an increased CENP-C and CENP-N recruitment to the array compared to targeting LacI-HJURP (Suppl. Figure 2-4 A-D). Targeting LacIHJURP ${ }^{\text {Scm3Conserved }}$, an HJURP fragment containing its N-terminal Scm3 domain and its vertebrate conserved domain (amino acids 1-348) (Sanchez-Pulido et al., 2009), recruited significantly more CENP-A to the LacO array (Suppl. Figure 2-4 E). Most of this CENPA was not stably incorporated, similarly to targeting the full length LacI-HJURP protein (Suppl. Figure 2-4 E). Why deletion of the C-terminus of HJURP resulted in increased CENP-A recruitment to the array is not understood. It is possible the C-terminus of HJURP is required for ensuring the correct number of CENP-A molecules is recruited to a particular site, possibly through its self-dimerization or through its interaction with Mis $18 \alpha$. Recruiting this large amount of CENP-A to the LacO array was inhibitory to 
CCAN recruitment, reducing CENP-C and CENP-N percentages to 6 and 9\%, respectively, compared to 24 and $37 \%$ when full length LacI-HJURP was targeted (Suppl. Figure 2-4 A-D). These data emphasize the need for stable, CENP-A nucleosome assembly in order to recruit CCAN members, even at non-centromeric locations. 
Figure 2-2. HJURP deposited CENP-A recruits constitutive centromere proteins

(A) LacO/TRE U2OS cells were transiently transfected with LacI-HJURP and constructs expressing LAP-tagged CENP-C, CENP-M, CENP-N, or CENP-T. Cells were preextracted and fixed 72 hours after transfection. The presence of CENP-A was assessed using antibodies against endogenous CENP-A. Insets show the arrays at 400X magnification. Scale bar $=5 \mu \mathrm{m}$. (B) LacI control images for LAP-CENP C-T, indicating that recruitment is never observed in the absence of CENP-A. Scale bar $=5 \mu \mathrm{m}$. (C) Graph showing the percentage of doubly transfected (GFP and LacI-HJURP) U2OS LacO/TRE cells with endogenous CENP-A present at the array that also recruited the indicated constitutive centromere proteins. ( $\geq 30$ cells per condition, bars represent S.D). 


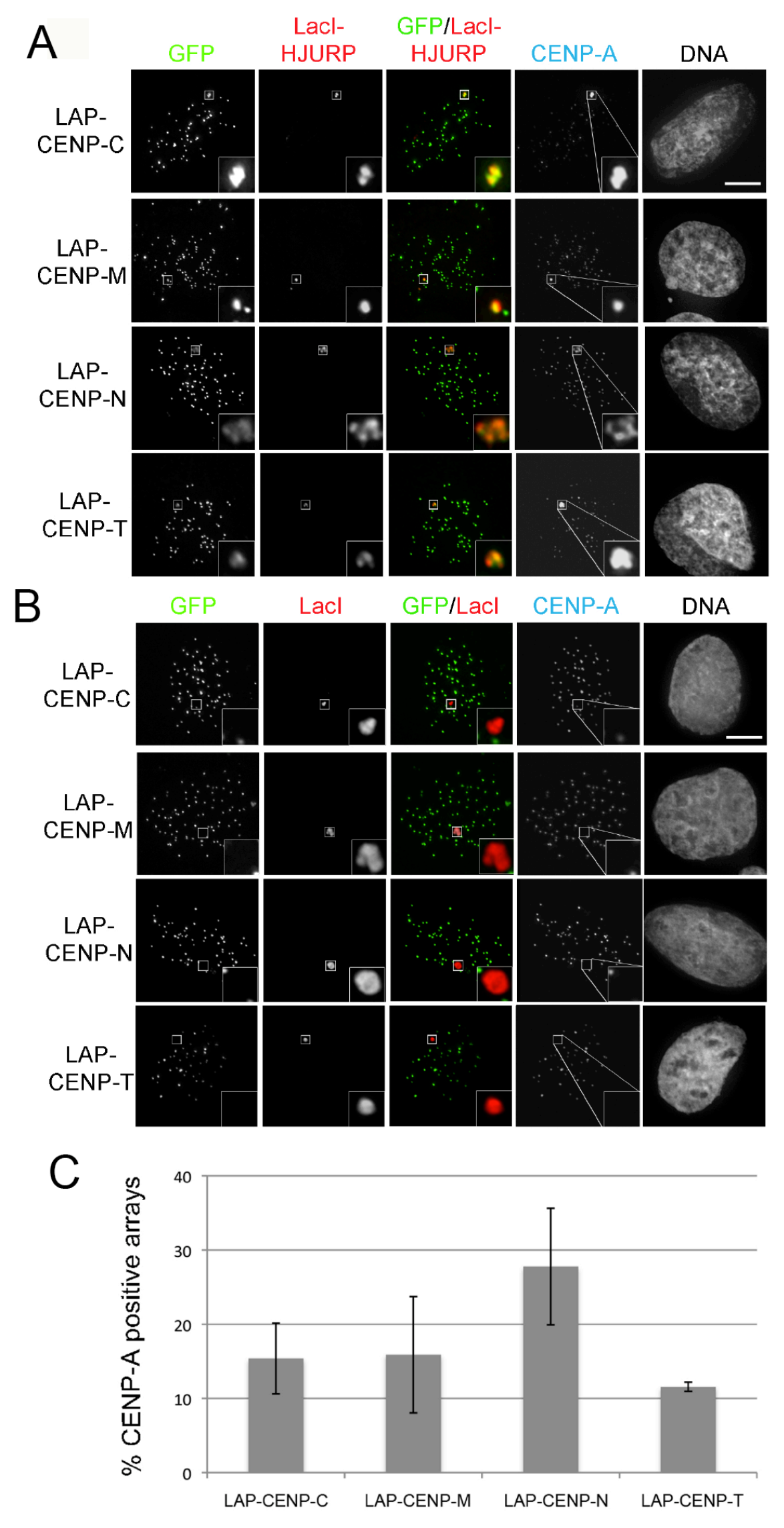




\section{Ectopic centromeres formed by HJURP act as kinetochores during mitosis}

The stable association of CENP-A at the array and the subsequent recruitment of constitutive centromere proteins prompted us to investigate whether mitosis-specific kinetochore proteins would also be recruited to the array. To address this, we immunostained mitotic chromosome spreads for the microtubule binding kinetochore protein NDC 80 and observed its recruitment to the LacO array in $40 \%$ of cells transfected with LacI-HJURP ${ }^{\mathrm{Scm} 3}$ (Figure 2-3 A). Spindle assembly checkpoint proteins, Aurora B and $\mathrm{Mad} 2$, were also recruited to the $\mathrm{LacO}$ arrays when LacI-HJURP ${ }^{\mathrm{Scm} 3}$ was targeted (Suppl. Figure 2-5 A, B). Endogenous centromeres exhibit a unique morphology in mitotic chromosomes called a constriction. In the LacI-HJURP ${ }^{\mathrm{Scm} 3}$-transfected cells where NDC80 is recruited to the array, we observe a second region of constriction at the array, in addition to the constriction present at the endogenous centromere consistent with the array acting as a functional kinetochore (Figure 2-3 A).

Cells were treated with the Eg5 inhibitor Monastrol, to demonstrate the array behaves similarly to endogenous centromeres during mitosis. Under Monastrol treatment, cells fail to separate their spindle poles during prometaphase and arrest in mitosis with their centromeres oriented toward the central pole and their telomeres towards the periphery. The array of the U2OS cells is incorporated near the telomere of chromosome 1 (Janicki et al., 2004) and is located away from the central cluster of centromeres, identified by CENP-T staining in control cells (LacI transfected, Figure 2-3 B). However, when LacI-HJURP ${ }^{\mathrm{Scm} 3}$ is expressed we observe the array closer to the central cluster of centromeres at the middle of the monopolar mitotic structure, consistent with the array 
acting as a centromere and binding microtubules (LacI-HJURP ${ }^{\mathrm{Scm} 3}$ transfected, Figure 23 B).

We predicted that cells in which ectopic centromeres were successfully assembled at the LacO array should form stable microtubule attachments and cause errors in chromosome segregation due to the presence of two active centromeres on a single chromosome. As expected, cold-stabilized microtubules, characteristic of kinetochore fibers (Brinkley and Cartwright, 1975), were observed to terminate at several LacO arrays assembled by LacI-HJURP ${ }^{\mathrm{Scm} 3}$ in metaphase cells (Figure 2-3 C). Consistent with an interaction between microtubules of the mitotic spindle and the LacO array, cells transfected with LacI-HJURP ${ }^{\mathrm{Scm} 3}$ exhibited lagging chromosomes during anaphase (Figure 2-3 D). The lagging chromosomes contain the LacO array (Figure 2-3 E), which suggests that the array of each chromosome is interacting with microtubules emanating from both spindle poles in a merotelic-like arrangement. These data support our findings that HJURP-assembled ectopic centromeres at the array can mimic centromeric CENP-A nucleosomes through their ability to recruit CENP-A ${ }^{\mathrm{NAC}}$ proteins and assemble a functioning kinetochore. 
Figure 2-3. Recruitment of CENP-A by HJURP $P^{\mathrm{Scm} 3}$ mediates kinetochore formation at the LacO array.

(A) Mitotic chromosome spreads from U20S-LacO/TRE cells transfected with LacI or LacI-HJURP $^{\mathrm{Scm} 3}$, arrested in nocodazole, and stained with antibodies for NDC80. $40 \%$ of LacI-HJURP arrays recruited NDC80. Scale bar $=5 \mu \mathrm{m}$. (B) Monastrol-arrested cells transfected with LacI or LacI-HJURP ${ }^{\mathrm{Scm} 3}$ and immunostained for centromere marker CENP-T. Radial distribution plots describe the average centromere position (black circle) in the cells measured ( $>26$ cells per condition) relative to the center of the DNA mass. The array position is diagrammed relative to the center of the DNA mass as blue triangles (LacI) or red diamonds (LacI-HJURP ${ }^{\mathrm{Scm} 3}$ ). The gray circle represents one standard deviation from the average centromere position. The LacI-HJURP ${ }^{\mathrm{Scm} 3}$ array falls within the centromere region in $69 \%$ of transfected cells, versus $15 \%$ for LacI controls (C) Selective stabilization of kinetochore bound microtubules through cold-treatment demonstrates the LacI-HJURP ${ }^{\mathrm{Scm} 3}$ arrays form stable microtubule interactions similar to endogenous centromeres. Scale bar $=5 \mu \mathrm{m}$. (D) LacO-SceI-Tet NIH3T3 cells were transfected with YFP-histone H2B and followed by live-cell imaging as they progress through mitosis. Times are given relative to the last frame when cells were in metaphase. Arrows indicate the array, asterisk indicates non-chromatin bound, unspecific LacI staining. LacI arrays segregated normally $100 \%$ of the time. Scale bar $=5 \mu \mathrm{m}$. (E) Insets taken from images in (D) show the behavior of the array (red in merge) and YFP-H2B (green in merge) for $(1,2)$ LacI-HJURP $\mathrm{Scm}^{\mathrm{Sc} 3}$ at $6 \mathrm{~min}$. and $9 \mathrm{~min}$. into anaphase, respectively. Scale bar $=2 \mu \mathrm{m}$. 


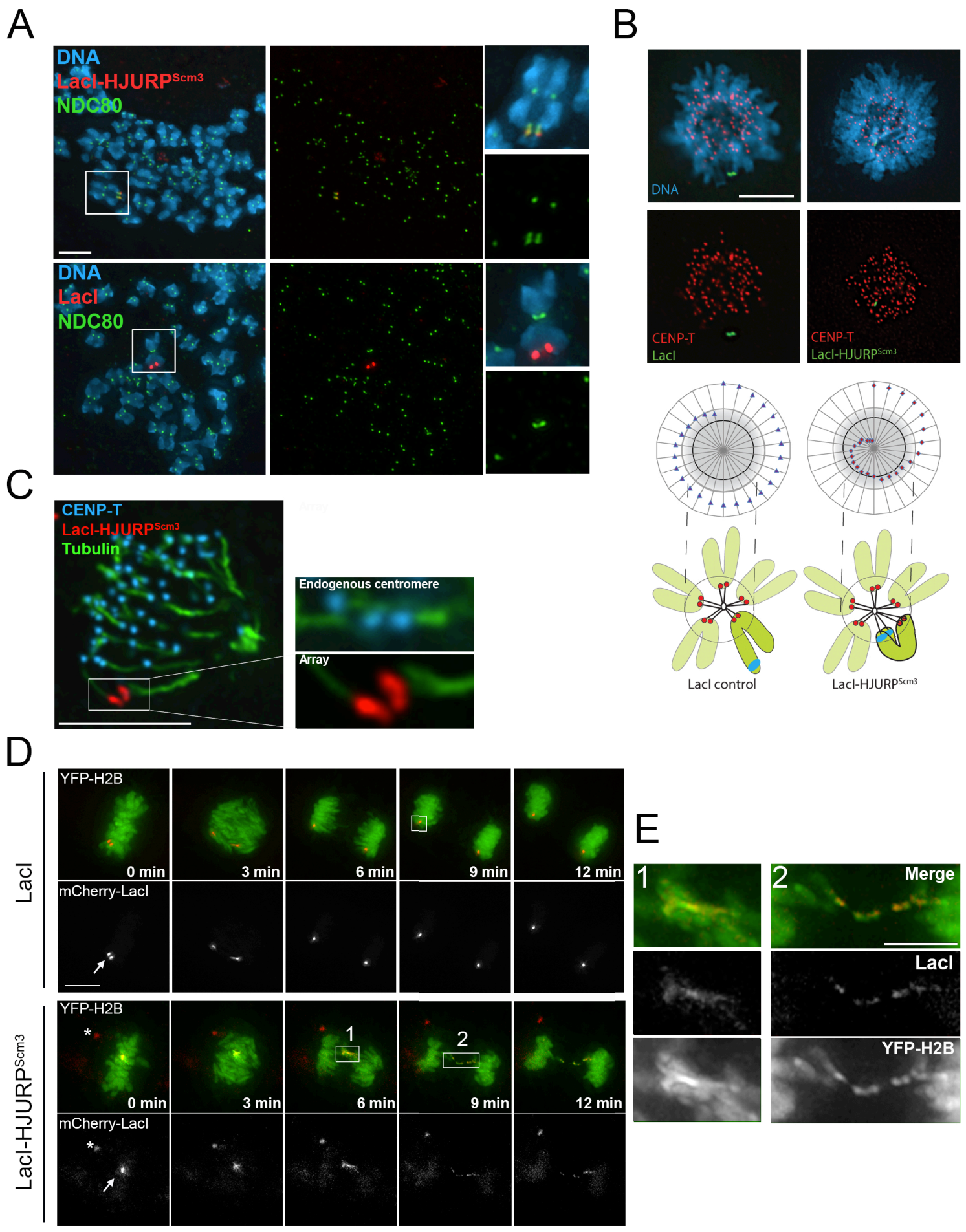


In vitro assembly of CENP-A nucleosomes by HJURP $P^{\mathrm{Scm} 3}$

It is unclear whether HJURP is required only to stabilize and deliver prenucleosomal CENP-A to centromeres or if HJURP is also actively involved in the assembly of CENP-A nucleosomes at the centromere. Based on the ability of HJURP to drive the stable association of CENP-A at the LacO array, we hypothesized that HJURP plays an active role in the deposition of CENP-A nucleosomes at centromeres. We assessed the ability of HJURP to assemble CENP-A containing nucleosomes in an in vitro chromatin assembly assay using purified recombinant proteins, independent of other assembly factors. The assembly of nucleosomes in this assay is assessed on closed circular plasmid DNA by monitoring the formation of topoisomers (Lusser and Kadonaga, 2004).

Since the Scm3 domain of HJURP is able to bind CENP-A, and we demonstrated the stable association of CENP-A at the LacO array in response to LacI-HJURP ${ }^{\mathrm{Scm} 3}$ expression (Figure 2-1 D; Suppl. Figure 2-4 E), we reasoned that this fragment of HJURP should be sufficient to assemble CENP-A nucleosomes. MBP-tagged HJURP ${ }^{\mathrm{Scm} 3}$, containing amino acids 1-208, was expressed in bacteria and purified (Suppl. Figure 2-6 A). As hypothesized, when incubated with CENP-A and histones H4, H2A, and H2B, HJURP $^{\mathrm{Scm} 3}$ was indeed able to assemble CENP-A nucleosomes as indicated by the accumulation of faster migrating topoisomers (Figure 2-4 A). The accumulation of fully supercoiled plasmids increased with increasing the amounts of HJURP ${ }^{\mathrm{Scm} 3}$ (Figure 2-4 AC). HJURP-assembled CENP-A nucleosomes protected the expected $\sim 150$ bps of DNA after micrococcal nuclease digest (Figure 2-4 D).

General histone chaperones are often promiscuous in their ability to bind and 
assemble histones into nucleosomes as exemplified by dNAP, which is capable of directly interacting with all four core histones and has been shown to assemble both histone H3 and CENP-A chromatin (Figure 2-5A; Suppl. Figure 2-6 B) (Park and Luger, 2006; Yoda et al., 2000). We next examined whether the assembly activity of MBPHJURP $^{\mathrm{Scm} 3}$ was specific for CENP-A containing nucleosomes. MBP-HJURP ${ }^{\mathrm{Scm} 3}$ was not able to efficiently assemble nucleosomes when CENP-A was replaced with histone H3.1 (Figure 2-4 A-C), consistent with the inability of HJURP to bind histone H3-H4 (Foltz et al., 2009) and supporting its role as a CENP-A specific assembly factor. While the degree of assembly of CENP-A nucleosomes correlates with the amount of HJURP ${ }^{\mathrm{Scm} 3}$ present in the reaction, the limited amount of supercoiling observed in $\mathrm{H} 3$ assembly reactions did not increase as increasing amounts of $\mathrm{HJURP}^{\mathrm{Scm} 3}$ were titrated into the reactions (Figure 2-4 B, C). This suggests the limited degree of supercoiling observed with histone $\mathrm{H} 3$ does not reflect chaperone-mediated assembly. Together these data support the hypothesis that HJURP is a CENP-A specific chromatin assembly factor possessing the intrinsic ability to deposit CENP-A nucleosomes into DNA.

Since both HJURP and nucleophosmin1 (NPM1) are consistently co-purified with pre-nucleosomal CENP-A, and NPM1 has been shown previously to act as a histone chaperone for histone H3 nucleosomes (Okuwaki et al., 2001), we sought to determine whether NPM1 might contribute to CENP-A nucleosome deposition (Dunleavy et al., 2009; Foltz et al., 2009; Frehlick et al., 2007; Shuaib et al., 2010). NPM1 was expressed and purified from bacteria (Suppl. Figure 2-6 A) and eluted from a size exclusion column as a pentamer (Okuwaki et al., 2001). Like dNAP and HJURP ${ }^{\text {Scm3 }}$, NPM1 was also able to assemble CENP-A containing nucleosomes onto plasmid DNA (Figure 2-4 D; 2-5 A- 
D). When NPM1 and HJURP were both present in the assay we did not observe an increase in assembly efficiency (data not shown).

Cse4 in Saccharomyces cerevisiae is reported to form a centromeric subnucleosomal particle with histone $\mathrm{H} 4$ that lacks histones H2A and H2B and may include Scm3 (the homolog of HJURP) (Mizuguchi et al., 2007; Stoler et al., 2007; Williams et al., 2009). Sub-nucleosomal H3/H4 heterotetramer complexes can be deposited onto DNA templates resulting in supercoiling (Peterson, 2007), and recently, Shuaib et al. (2009) demonstrated the ability of HJURP to deposit a single CENP-A/H4 heterotetramer into DNA. We determined if HJURP ${ }^{\mathrm{Scm} 3}$ was able to assemble extended arrays of CENPA/H4 heterotetramers into plasmid DNA and to compare the extent of supercoiling induced by heterotetramer assembly relative to octameric CENP-A assembly. We observed plasmid supercoiling around CENP-A/H4 heterotetramers in response to all three assembly factors tested; although, in each case, the degree of supercoiling was less than that observed with CENP-A octamers, which included H2A and H2B (Figure 2-5 AC, Suppl. Figure 2-6 C). When the amount of CENP-A/H4 heterotetramer was increased by 2 -fold in the reaction over the amount in the octamer assemblies, dNAP became as efficient as in the presence of all four histones. However, NPM1 and HJURP ${ }^{\mathrm{Scm} 3}$ continued to yield a lesser degree of plasmid supercoiling around the CENP-A/H4 heterotetramer (Figure 2-5 B, C; Suppl. Figure 2-6 C). These observations are consistent with either a lesser activity of HJURP ${ }^{\mathrm{Scm} 3}$ for the assembly of CENP-A containing heterotetramers or with a decreased degree of supercoiling per heterotetramer.

It has been reported that CENP-A containing nucleosomes positively supercoil DNA in Drosophila and S. cerevisiae, in contrast to the negative supercoiling produced 
by canonical histone H3 containing nucleosomes (Furuyama and Henikoff, 2009). We separated $\mathrm{HJURP}^{\mathrm{Scm} 3}$ induced topoisomers in the presence of chloroquine, an intercalating drug that allows for the detection of both positive and negative supercoiling by a shift in the topoisomers. Positively supercoiled DNA will shift towards faster migrating species in the presence of chloroquine, and negatively supercoiled DNA will shift towards more slowly migrating species (Lusser and Kadonaga, 2004). MBP$\mathrm{HJURP}^{\mathrm{Scm} 3}$-assembled CENP-A containing nucleosomes induced negative supercoils as indicated by the upward shift in the gel (Figure 2-5 D, lower panel), contrary to what has been observed for the Drosophila CID and budding yeast Cse4 nucleosomes. These data suggest human CENP-A nucleosomes assembled by their native chaperone include histones $\mathrm{H} 2 \mathrm{~A}$ and $\mathrm{H} 2 \mathrm{~B}$ and are wrapped in a left-handed direction, similar to canonical H3.1 containing nucleosomes. The crystal structure of the human CENP-A nucleosome confirms that it is octameric and wraps DNA in a left-handed manner (Tachiwana et al., 2011). 
Figure 2-4. HJURP $P^{S c m 3}$ is sufficient to assemble CENP-A nucleosomes in vitro.

(A) Plasmid supercoiling assays were conducted using recombinant MBP-tagged HJURP $^{\mathrm{Scm} 3}$ and recombinant CENP-A octamer (histones H4, H2A and H2B) or Histone H3.1 octamer to assess the relative ability of HJURP to assemble CENP-A and H3.1 containing nucleosomes. Relaxed DNA lane contains topoisomerase treated S.C. (supercoiled) plasmid DNA. HJURP ${ }^{\mathrm{Scm} 3}$ induced supercoiling more efficient in the presence of CENP-A relative to H3.1. (B) Line scans across topoisomers within conditions presented in (A). Lines indicate the least supercoiled topoisomers. Boxes indicate the location of the maximally assembled topoisomers. (C) Assembly reactions from (A) containing H3.1 (dark grey) and CENP-A (light gray) are graphed here as foldintensity over reactions containing no HJURP ${ }^{\mathrm{Scm} 3}$. (D) Assembly reactions in A (using HJURP $^{\mathrm{Scm} 3}$ ) or assembly reactions using NPM1 digested with MNase to show DNA protection of the assembled species.

All data in Figure 2-4 were collected and assembled by Henning Kuich and Dan Foltz. 
A

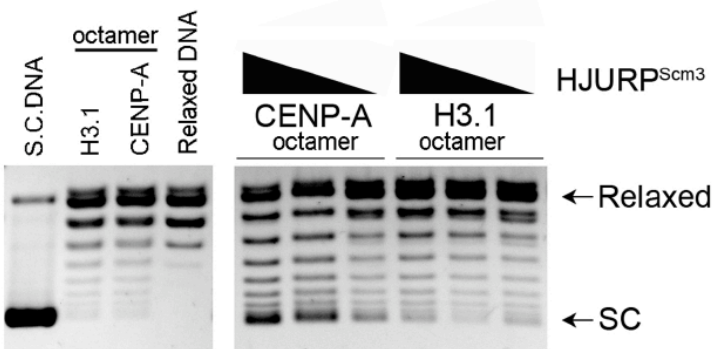

B

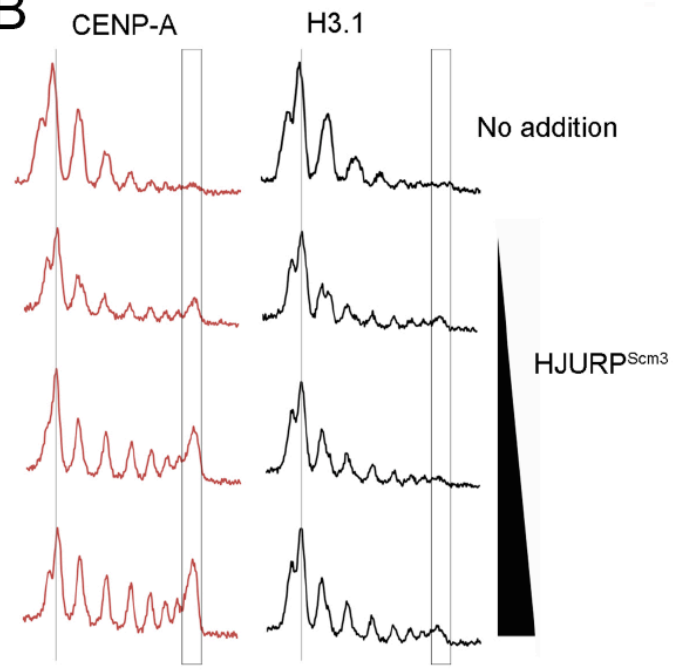

C

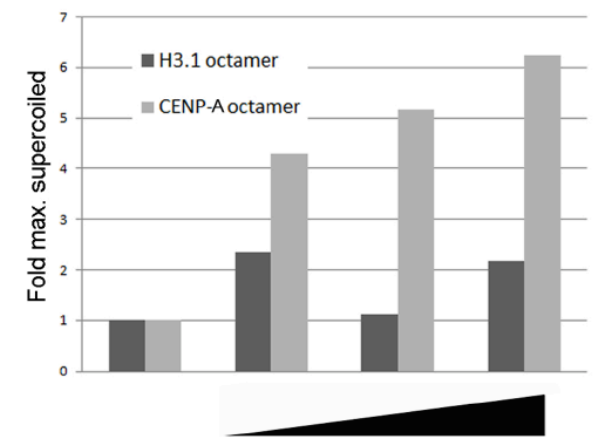

D

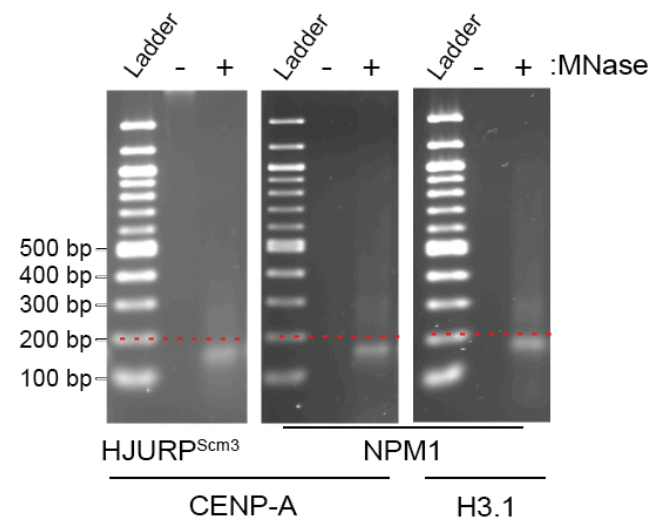


Figure 2-5. HJURP $P^{S \mathrm{~cm} 3}$ assembled CENP-A nucleosomes are negatively supercoiled and contain $H 2 A$ and $H 2 B$.

(A) Supercoiling assay comparing assembly efficiencies of chaperones dNAP, NPM1, and HJURP ${ }^{\mathrm{Scm} 3}$ with CENP-A histone octamers (CENP-A/H4, H2A/H2B) in (A) or with CENP-A/H4 alone in (B). CENP-A/H4 levels added to the reactions were varied from $1 \mathrm{X}$ to $2 \mathrm{X}$ compared with the amount of CENP-A/H4 present in the reactions in (A). Line scans are presented in Figure S4. (C) Integrated intensities of maximally supercoiled populations were measured from reactions in (A) and (B). Values are graphed as foldmaximally supercoiled heterotetramer to octamer. (D) Supercoiling assay showing assembly activities (upper panel) for dNAP, HJURP ${ }^{\mathrm{Scm} 3}$, and NPM1. Supercoiled DNA was separated by agarose gel electrophoresis with (lower panel) or without (upper panel) the DNA intercalating agent chloroquine to distinguish negatively and positively supercoiled DNA.

All data in Figure 2-5 were collected and assembled by Henning Kuich and Dan Foltz. 

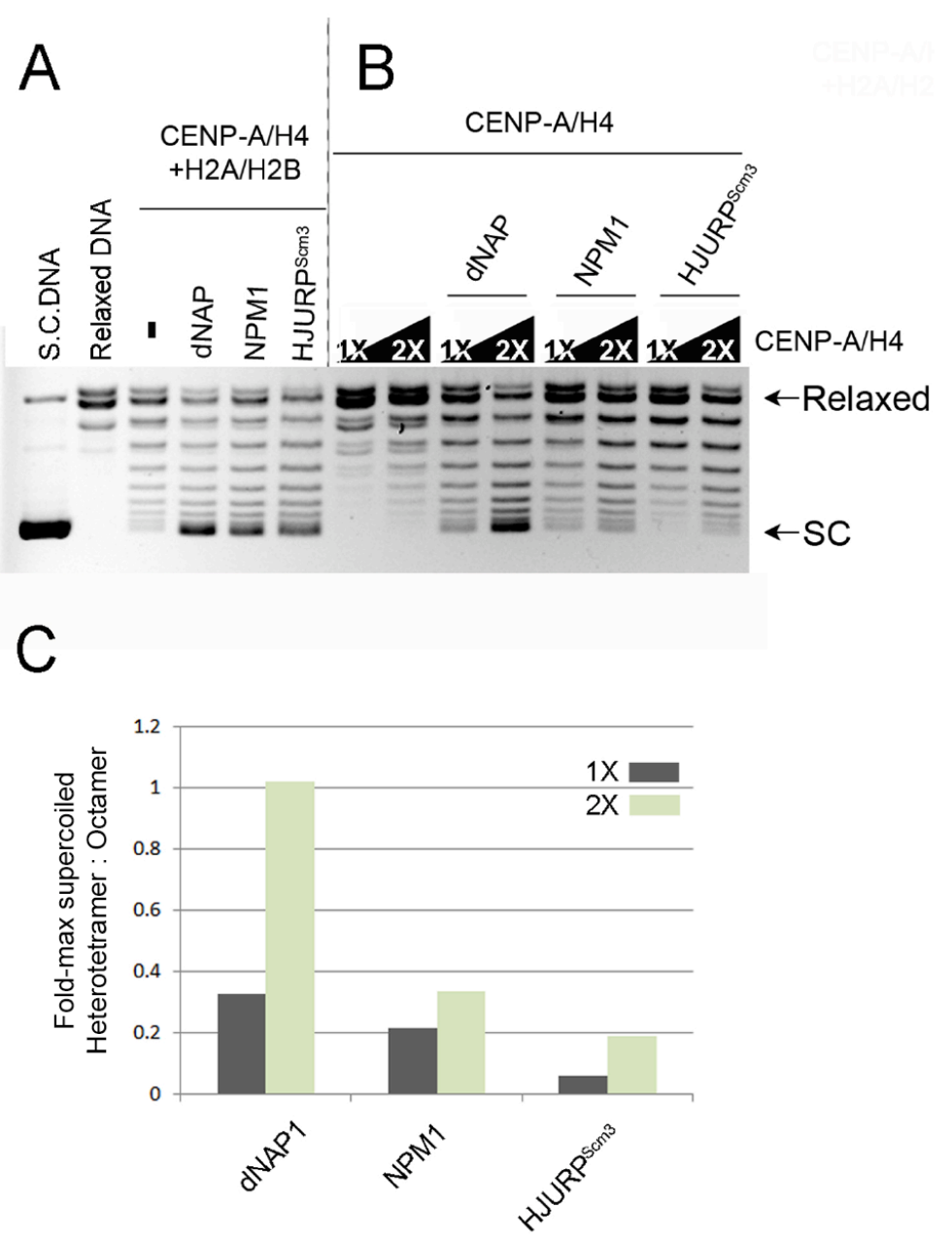

D

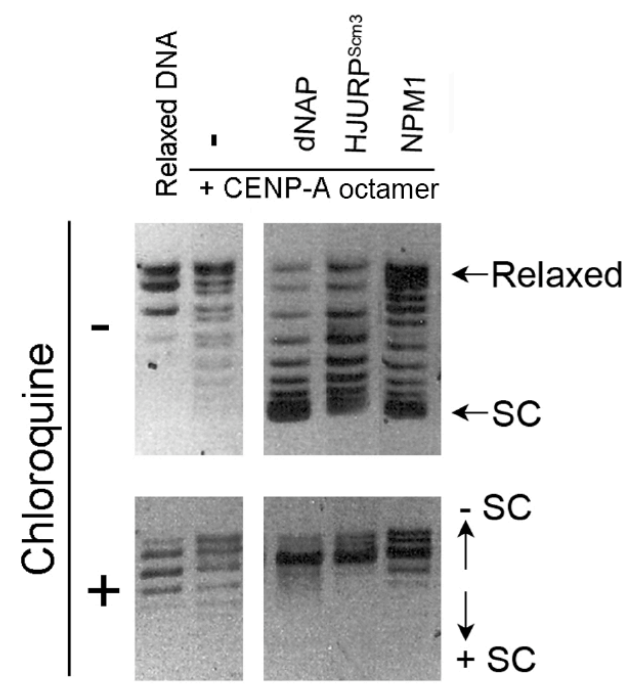


HJURP Centromeric Localization is Dependent on the Mis 18 Complex at Centromeres

HJURP and the Mis18 complex are both required for the recruitment of newly synthesized CENP-A to the centromere; however, the function of the Mis18 complex in CENP-A assembly remains unclear. It was not known whether Mis18 and HJURP are required for independent events in CENP-A deposition, or whether the action of the Mis18 complex is required for HJURP recruitment. To address this question Mis18 $\alpha$, Mis $18 \mathrm{BP} 1^{\text {hsKNL2}}$, or HJURP were knocked down by siRNA treatment in HeLa cell lines that stably express either GFP-tagged Mis18 $\alpha$ or GFP-tagged HJURP. The GFP-tagged proteins behave similarly to endogenous proteins, as the proportion of cells with centromeric GFP-Mis18a or GFP-HJURP increases significantly when we enrich for cells in early G1 (Suppl. Figure 2-7).

Knockdown of Mis18 $\alpha$ by siRNA reduced GFP-Mis18 $\alpha$ protein levels to below $25 \%$ of mock treated levels after 48 hours (Figure 2-6 A). HJURP siRNA reduced endogenous HJURP to below $25 \%$ of normal cellular levels while also substantially decreasing the level of the GFP-tagged protein (Figure 2-6 B). Centromeric localization of GFP-tagged Mis18 $\alpha$ was abolished following Mis18 $\alpha$ siRNA treatment (Figure 2-6 C, D). Antibodies that recognize CENP-T were used to identify centromeres. Treating cells with siRNA against Mis18BP1 $1^{\text {hsKNL2 }}$ did not lower the protein level of the exogenous GFP-Mis18a (Figure 2-6 A) but did abolish Mis18 $\alpha$ localization at centromeres (Figure 2-6 C, D) consistent with previous findings (Fujita et al., 2007).

HJURP siRNA completely eliminated centromeric localization of the GFP-tagged HJURP protein after 48 hours (Figure 2-6 E, F). In contrast, siRNA knockdown of HJURP did not alter the localization pattern of GFP-Mis18 $\alpha$ to centromeres as compared 
to the GAPDH control nor did it decrease the protein level of GFP-Mis18 $\alpha$ (Figure 2-6 A, C, D). Importantly, siRNA against either Mis $18 \alpha$ or Mis18BP $1^{\text {hsKNL2 }}$ eliminated HJURP recruitment to centromeres (Figure 2-6 E, F), while not affecting the level of endogenous or GFP-tagged HJURP (Figure 2-6 B). Therefore, the Mis18 complex is required for HJURP recruitment to centromeres suggesting that the lack of CENP-A deposition in Mis18 knockdown experiments results from the inability to recruit HJURP (Fujita et al., 2007; Maddox et al., 2007).

The Mis18 complex is required for the recruitment of HJURP to centromeres (Figure 2-6), and this is consistent with the previous observation that Mis18 is required for the recruitment of new CENP-A to centromeres (Fujita et al., 2007; Maddox et al., 2007). To determine if the Mis 18 complex is also required for the function of HJURP in recruiting and stabilizing CENP-A, we performed siRNA knockdown of Mis18BP1 ${ }^{\text {hsKNL2 }}$ in the $\mathrm{LacO}$ containing $\mathrm{U} 2 \mathrm{OS}$ cells. Mis18BP1 ${ }^{\mathrm{hKKNL} 2}$ protein was reduced to less than $10 \%$ of endogenous levels by siRNA treatment of U20S cells as indicated by immunoblot and by the loss of centromeric CENP-A (Figure 2-7 A, B). The stability of endogenous CENP-A at the LacO array in LacI-HJURP ${ }^{\mathrm{Scm} 3}$ transfected cells was unaffected by Mis18BP1 $1^{\text {hKKNL2 }}$ depletion following IPTG treatment (Figure 2-7 A, C). Similar numbers of CENP-A containing arrays were observed in GAPDH and Mis18BP1 $1^{\text {hsKNL2 }}$ siRNA treated cells following treatment with IPTG (Figure 2-7 C). We conclude the requirement for the Mis 18 complex can be bypassed by directly targeting HJURP to DNA. 
Figure 2-6. Recruitment of HJURP to Centromeres Requires the Mis18 Complex.

Cellular extracts from siRNA treated and control cell lines were analyzed by western blot using (A) anti-GFP antibody or (B) anti-HJURP antibodies. Each lane contains lysate from $1 \times 10^{5}$ cells. Dilution series were generated from mock treated (A) HeLa GFPMis18 $\alpha$ or (B) parental HeLa cells. (C) Stable GFP-Mis18a cells lines were treated with siRNA against Mis18a, Mis18BP1 ${ }^{\text {hsKNL2 }}$, HJURP or GAPDH (control). Representative images of siRNA treated GFP-Mis18a (green) cells were selected in which a midbody was clearly present (DIC, arrow) to show the cell was in early G1. DAPI staining was overlaid onto the DIC image. Cells were stained with anti-CENP-T (Red). Scale bar $=$ $5 \mu \mathrm{m}$. (D) Average percentage of GFP-Mis $18 \alpha$ centromere-positive nuclei from a population of $\geq 57$ cells in each siRNA treatment from two experiments. Error bars show the standard deviation between experiments. (E) Similar image acquisition as in (C), here stable HeLa GFP-HJURP cells were treated with the same siRNA. Scale bar $=5 \mu \mathrm{m}$. (F) Average percentage of GFP-HJURP centromere-positive nuclei from a population of $\geq 135$ cells in each siRNA treatment from two experiments.

All data in Figure 2-6 were collected and assembled by Madison Stellfox. 

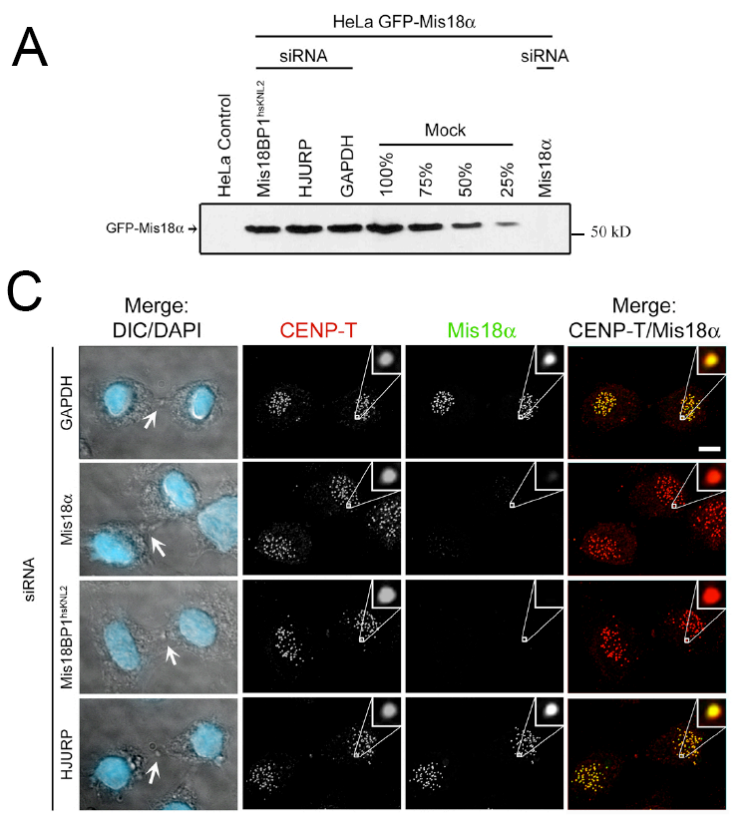

D

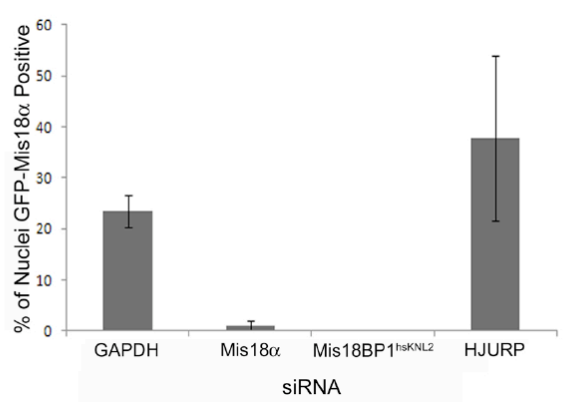

B

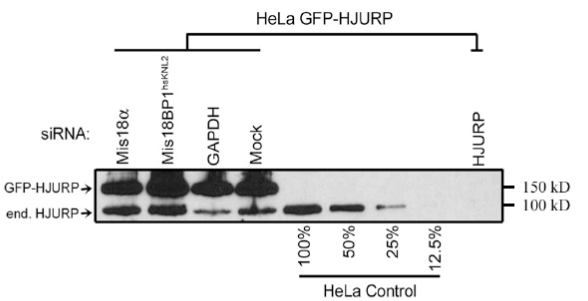

E

Merge:

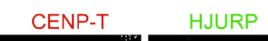

Merge: CENP-T/Mis18
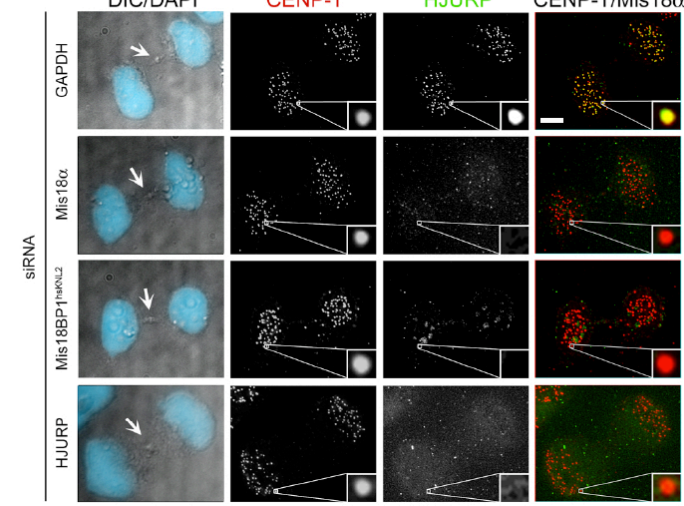

F

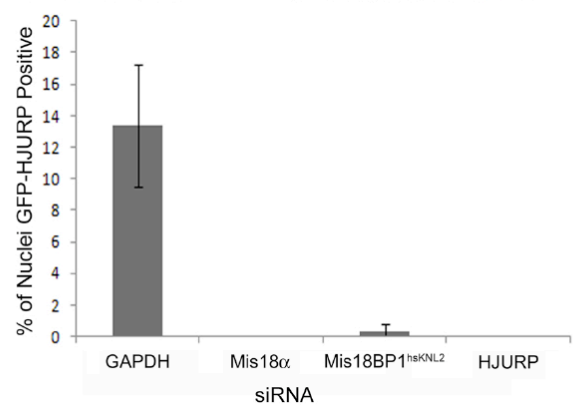


Figure 2-7. The Mis 18 complex is not required for CENP-A deposition at the LacO/TRE array.

(A) Representative images of endogenous CENP-A recruitment in U2OS-LacO cells treated with $15 \mathrm{mM}$ IPTG after 72 hours of either GAPDH or Mis18BP1 $1^{\text {hKKNL2 }}$ siRNA treatment. Cells had been transiently transfected with LacI-HJURP ${ }^{\mathrm{Scm} 3}$ and GFP-TetR 48 hrs prior to fixation. Cells were transfected after an initial $24 \mathrm{hr}$ siRNA treatment in order to ensure Mis18BP $1^{\text {hsKNL2 }}$ depletion prior to CENP-A establishment at the array. Scale bar represents $5 \mu \mathrm{m}$. (B) Cellular extracts from GAPDH and Mis18BP1 ${ }^{\text {hsKNL2 }}$ siRNA treated cells were analyzed by western blot using an anti-Mis18BP1 $1^{\text {hKKNL2 }}$ antibody. Each lane contains lysate from $1 \times 10^{7}$ cells. (C) Quantification of CENP-A staining at the LacO/TRE array marked by GFP-TetR after $72 \mathrm{hrs}$ of GAPDH or Mis18BP1 ${ }^{\text {hsKNL2 }}$ siRNA treatment and $1 \mathrm{hr}$ of $15 \mathrm{mM}$ IPTG treatment. $\geq 30$ cells per condition, $\mathrm{n}=2$, error bars represent the standard deviation between the two experiments. P-value between GAPDH and Mis18BP $1^{\text {hsKNL2 }}$ is 0.3609 . 


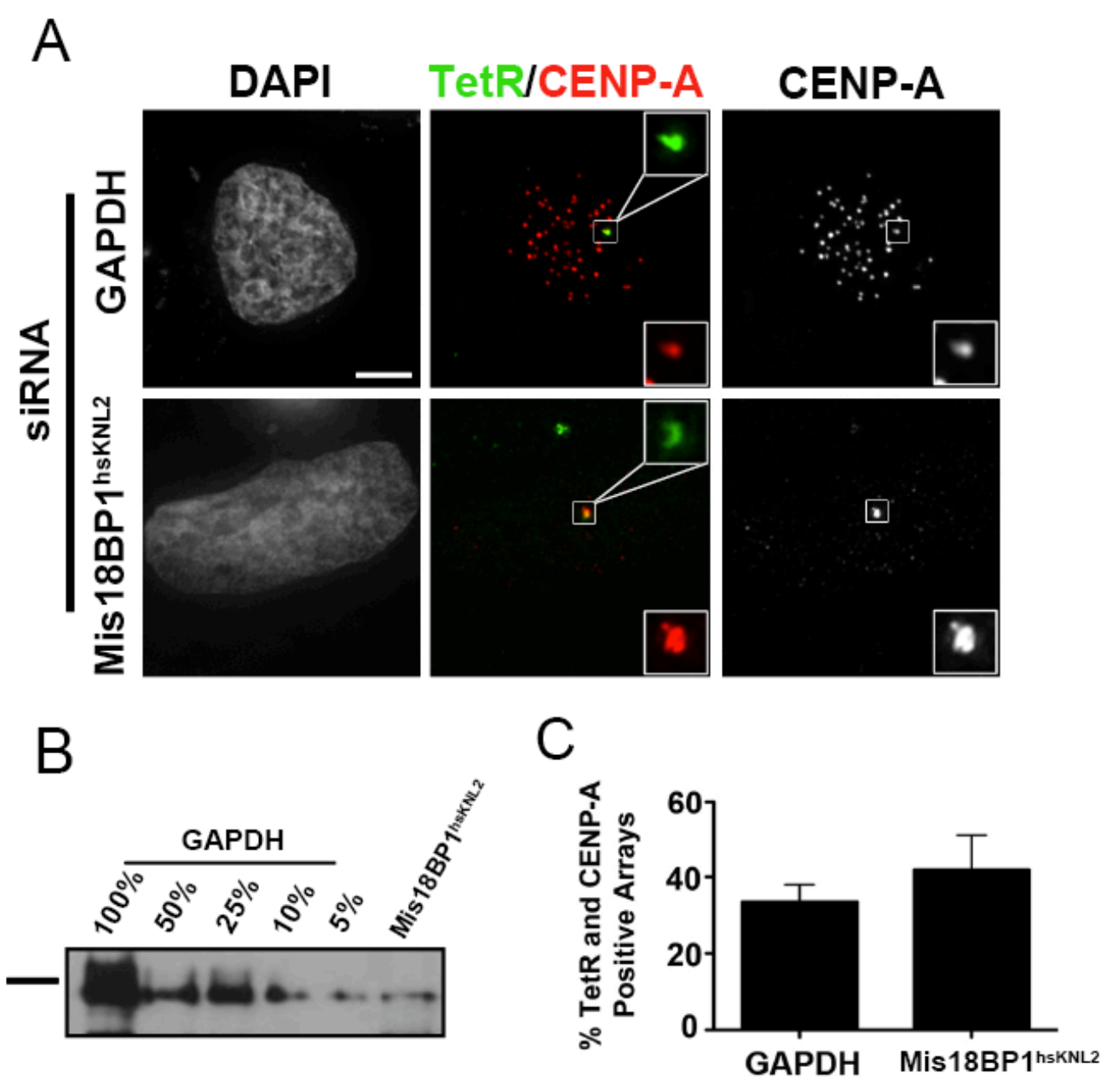


Figure 2-8. HJURP recruitment determines centromere position.

(A) HJURP is recruited to centromeres through the action of Mis18. During G1, HJURP directly assembles CENP-A nucleosomes at centromeres along with histones H2A and H2B. The recruitment of HJURP is the critical step in determining the site of the centromere. (B) Redirecting HJURP to an integrated LacO array bypasses the requirement for Mis18 and results in deposition of CENP-A and is sufficient to form a functional kinetochore. 


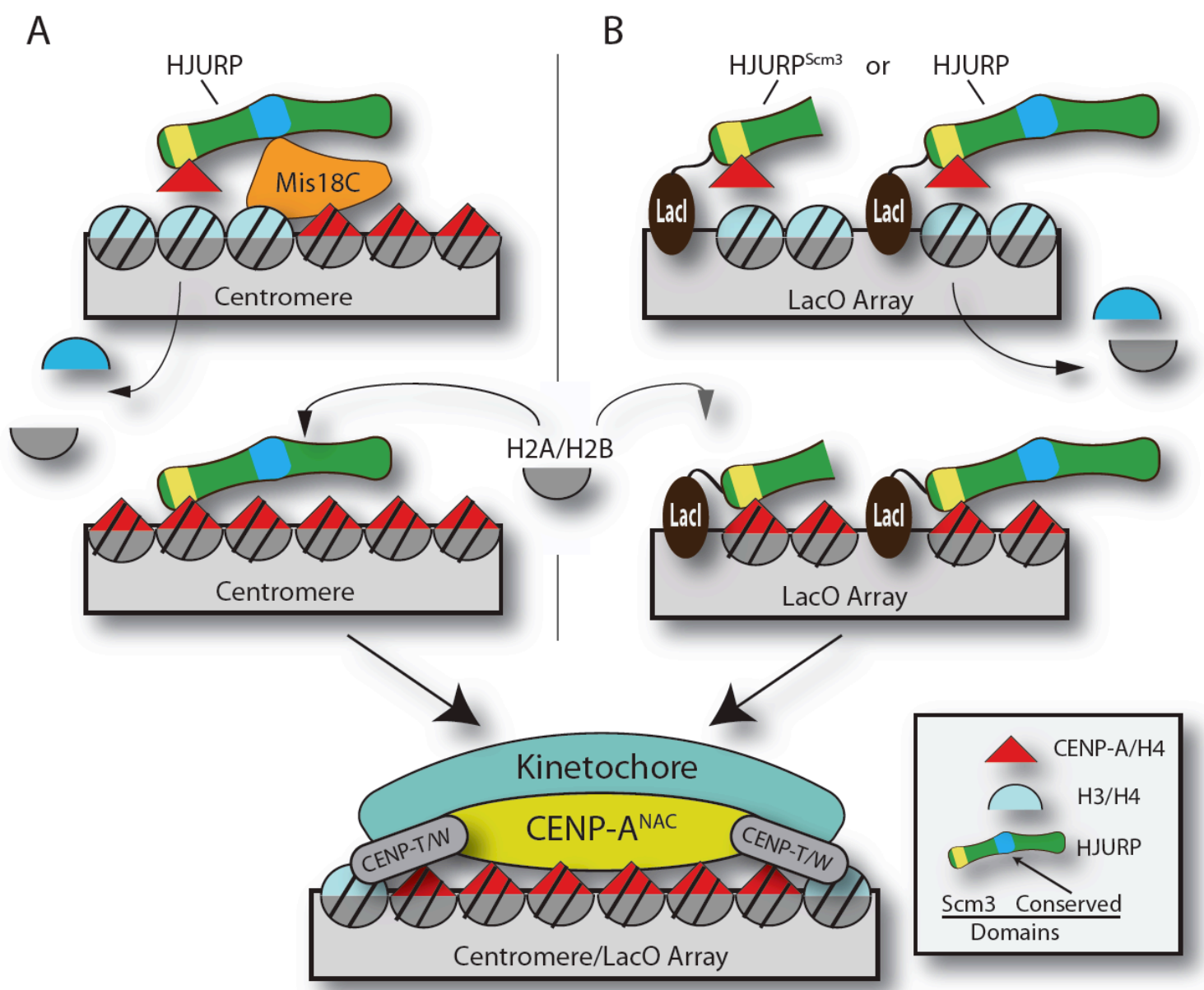




\section{$\underline{\text { Discussion }}$}

Determination of the site of centromere assembly is an epigenetic process that depends on the Mis 18 complex and the CENP-A specific chaperone HJURP during early G1. Here we have demonstrated that HJURP targeting to the centromere requires the Mis18 complex. By targeting HJURP to an ectopic location using the LacI/LacO system, we have bypassed the need for Mis18-mediated centromere recruitment of HJURP in order to assess the role of HJURP in CENP-A deposition independently of the context of the endogenous centromere. Targeting HJURP to the LacO array demonstrates that the recruitment of HJURP is the process that determines the site of CENP-A nucleosome deposition and subsequently the assembly of the associated constitutive centromere and mitotic kinetochore (Figure 2-8). Furthermore, we have determined HJURP is directly responsible for CENP-A nucleosome assembly in vitro. Together these data demonstrate HJURP recognizes the centromere through the action of the Mis 18 complex and that HJURP's intrinsic ability to assemble CENP-A nucleosomes is an integral step in the epigenetic mechanism by which centromeres are stably propagated.

The CENP-A nucleosome is independently recognized by two components of the CENP-A $^{\mathrm{NAC}} / \mathrm{CCAN}$, CENP-N and CENP-C (Carroll et al., 2010; Carroll et al., 2009) and both of these proteins are recruited to the LacO arrays containing CENP-A (Figure 2-2, Suppl. Figure 2-4). In vitro, CENP-N is unable to bind the CENP-A/H4 heterotetramer but uniquely recognizes the CENP-A-containing nucleosome assembled with histone H4, $\mathrm{H} 2 \mathrm{~A}$, and $\mathrm{H} 2 \mathrm{~B}$. The recruitment of CENP-N to the $\mathrm{LacO}$ array indicates the presence of assembled CENP-A nucleosomes. Increased CENP-N recruitment observed when LacI- 
HJURP $^{\mathrm{Scm} 3}$ is targeted also supports a need for the presence of assembled CENP-A nucleosomes at the array since LacI-HJURP ${ }^{\mathrm{Scm} 3}$ CENP-A levels at the array were the most stable following IPTG treatment (Figure 2-2, Suppl. Figure 2-4). The CENP-T/W complex, is localized in close proximity to the CENP-A nucleosome at centromeres, although it has been proposed to interact directly with histone $\mathrm{H} 3$ chromatin at the centromere (Foltz et al., 2006; Hori et al., 2008a). We observed CENP-T recruitment to the array suggesting the incorporation of CENP-A is able to organize the array relative to surrounding $\mathrm{H} 3$ nucleosomes in a way that reflects the arrangements present at endogenous centromeres (Figure 2-2). It was demonstrated the same year this work was published that recruitment of CENP-T and CENP-C are sufficient to build an ectopic centromere using the LacO system (Gascoigne et al., 2011). Our work is consistent with this as CENP-T and CENP-C were recruited to the CENP-A containing arrays that went on to assemble functional kinetochores (Figure 2-3).

Since this work was published, other groups have also employed this system for assaying centromere and kinetochore formation in flies and plants (Chen et al., 2014; Mendiburo et al., 2011; Teo et al., 2013). Tethering LacI-CENH3/CID in Drosophila cells is sufficient to epigenetically establish a kinetochore at a non-centromeric site and induce epigenetic recruitment and spreading of endogenous CENH3/CID to the noncentromeric site in subsequent generations (Mendiburo et al., 2011). Recently, similar results were demonstrated by tethering the CENH3/CID assembly factor, CAL1, or CENP-C to non-centromeric chromatin in Drosophila cells (Chen et al., 2014). Lastly, as mentioned above, this system was used to assemble a de novo kinetochore without the underlying CENP-A chromatin by tethering CENP-C and CENP-T together at a $\mathrm{LacO}$ 
array in human cells. While a de novo kinetochore did form, the site at which it formed was not heritable because it lacked underlying CENP-A chromatin (Gascoigne et al., 2011). This is in contrast to the establishment of centromeric chromatin shown here, which was able to epigenetically recruit CENP-A to the non-centromeric site in subsequent generations (Suppl. Figure 2-2 B). This was elegantly shown in Drosophila by two groups that demonstrated stable inheritance of the de novo centromere out to 5 days in S2 cells and 28 days using an episomal LacO plasmid (Chen et al., 2014; Mendiburo et al., 2011).

Data examining the ability of CENP-A over-expression to drive centromere and kinetochore formation have been somewhat mixed. In human cell lines, overexpression of CENP-A can lead to incorporation of CENP-A throughout the chromatin with an accompanying relocalization of CENP-C; however, these regions do not support kinetochore formation during mitosis (Van Hooser et al., 2001). Recruitment of CENP-A to the $\mathrm{LacO}$ array is successful at reconstituting centromere and kinetochore activity. This may be due to an enrichment of CENP-A at the LacO arrays relative to that deposited into general chromatin by overexpression. Alternatively, the kinetochore activity of the LacO array may reflect a contribution of HJURP-mediated assembly on the CENP-A nucleosomes at the array, as opposed to overexpression-induced chromatin incorporation of CENP-A, which may occur through HJURP-independent mechanisms. We have done preliminary studies to address this using a fusion protein of $\mathrm{HJURP}^{\mathrm{Scm} 3}$ and $\mathrm{H} 2 \mathrm{~B}$. Similarly to overexpression of CENP-A (Van Hooser et al., 2001), tethering HJURP ${ }^{\mathrm{Scm} 3}$ H2B all over chromosome arms by fusing it to H2B caused both CENP-A and CENP-C to also mis-localize along chromosome arms in both mitotic and interphase cells (Suppl. 
Figure 2-8). Interestingly, Hecl also mis-localized along chromosome arms with CENP$\mathrm{A}$ and CENP-C in mitotic spreads, arguing for some successful CENP-A assembly by HJURP ${ }^{\mathrm{Scm} 3}-\mathrm{H} 2 \mathrm{~B}$ inducing kinetochore component recruitment (Suppl. Figure 2-8 A-C). Future studies using this construct may be able to determine if CENP-A deposition along chromosome arms has "hot-spots" for where it is likely to deposit in human cells, similarly to what has been characterized in fission yeast and chickens (Hori et al., 2013; Ishii et al., 2008; Ogiyama et al., 2013).

The overexpression of CID in Drosophila was more successful in recapitulating centromere and kinetochore formation than overexpression in human cell lines. In this case, CID overexpression resulted in its accumulation throughout the chromatin and the assembly of centromere-like regions that recruited CENP-C and mediated microtubule attachments. These ectopic centromeres were restricted to a subset of sites within the chromatin (Heun et al., 2006). In our experiments where CENP-A deposition is restricted to a single location along the chromosome, we observed that not all cells recruited the constitutive centromere proteins and assembled kinetochores at the CENP-A assembled array, though this percentage was increased when $\mathrm{HJURP}^{\mathrm{Scm} 3}$ was tethered instead of LacI-HJURP full length (Suppl. Figure 2-4). The restricted recruitment of CENP-A NAC proteins to the array may reflect differences in the state of the underlying chromatin. Transcriptional activity or modification state of chromatin can negatively impact centromere formation in human artificial chromosomes (Bergmann et al., 2011; Nakano et al., 2008) and may inhibit the ability of HJURP to deposit CENP-A, acting as way to avoid spurious CENP-A deposition into non-centromeric loci. Alternatively a stepwise maturation of the CENP-A containing region toward a functional centromere may require 
multiple cell cycles and depend on cell cycle specific assembly of centromere proteins. Likewise, additional chromatin remodeling steps following CENP-A nucleosome deposition mediated by RSF1 or MgcRacGAP could influence both long-term stability of CENP-A nucleosomes and the assembly of the constitutive centromere (Lagana et al., 2010; Perpelescu et al., 2009).

Histone $\mathrm{H} 3$ variants partner with distinct chaperone complexes to facilitate their different temporal and spatial incorporation within the genome. Histone H3.1 nucleosome incorporation is coupled to DNA synthesis through an interaction between the p150 subunit of the chromatin assembly factor complex (CAF1) and PCNA (Moggs et al., 2000; Shibahara and Stillman, 1999). The assembly of H3.3 containing nucleosomes occurs independently of DNA synthesis and is accomplished through HIRA, ATRX, and DAXX (Drane et al., 2010; Goldberg et al., 2010; Tagami et al., 2004; Wong et al., 2010). Here we demonstrate the activity of the CENP-A chaperone/assembly factor HJURP is coupled to the centromere through the recruitment by the Mis 18 complex. $S$. pombe contains a single Mis 18 protein that directly interacts with Scm3 (Pidoux et al., 2009). However, HJURP ${ }^{\mathrm{Scm} 3}$, which contains the regions of significant homology between Scm3 and HJURP, does not demonstrate centromeric localization on its own (Suppl. Figure 2-3) (Zasadzinska et al., 2013). Our lab has since shown that human HJURP centromere targeting requires the C-terminal HCTD1 (HJURP C-terminal domain 1), suggesting the mechanism of HJURP centromeric recruitment in vertebrates is different from Scm3 recruitment in yeast (Zasadzinska et al., 2013). The involvement of Mis18 in recruiting HJURP to centromeres may be through a priming mechanism as previously proposed or through a direct binding interaction as recently discovered (Nardi 
et al. Unpublished results 2014) (Fujita et al., 2007; Wang et al., 2014) or some combination of the two.

Several models for the composition of the CENP-A nucleosome have been proposed including tetrameric forms that contain a single copy of each histone as well as hexameric forms that lack $\mathrm{H} 2 \mathrm{~A}$ and $\mathrm{H} 2 \mathrm{~B}$ but incorporate the yeast HJURP homolog Scm3. In human cells HJURP is present at centromeres during early G1 when new CENP-A nucleosomes are being actively recruited. Similarly to previous reports using full-length HJURP (Shuaib et al., 2010), HJURP ${ }^{\mathrm{Scm} 3}$ is able assemble CENP-A/H4 heterotetramers into DNA to some degree (Figure 2-5 B, C); however, the assembly mediated by $\mathrm{HJURP}^{\mathrm{Scm} 3}$ is processive and maximally efficient in the presence of histones $\mathrm{H} 2 \mathrm{~A}$ and $\mathrm{H} 2 \mathrm{~B}$ (Figure 2-4 A-C; Figure 2-5 B, C. The assembled structure protects approximately 145 base pairs of DNA (Figure 2-4 D). These data suggest human HJURP assembles an octameric nucleosome. Consistent with a centromeric nucleosome containing two copies of CENP-A, disruption of the CENP-A dimerization interface precludes the ability of CENP-A to accumulate at centromeres (Bassett et al., 2012b; Camahort et al., 2009; Sekulic et al., 2010). Additionally, the crystal structure of the CENP-A nucleosome has since been published and revealed it is an octamer with two copies of CENP-A, H4, H2A, and H2B that wraps DNA in a left-handed manner, similarly to the canonical H3 octamer (Tachiwana et al., 2011). It is possible that following deposition by HJURP, further remodeling by additional factors may mediate the conversion of the centromeric nucleosome into the hexameric and/or tetrameric forms observed by others (Dalal et al., 2007; Dimitriadis et al., 2010; Furuyama and Henikoff, 2009; Mizuguchi et al., 2007). 
In addition to HJURP, several other chromatin assembly factors have been implicated in the assembly of the human centromeres. NPM1 is also able to assemble CENP-A (Figure 2-5) and histone H3 nucleosomes (Okuwaki et al., 2001). NPM1 is associated with the CENP-A/HJURP prenucleosomal complex (Dunleavy et al., 2009; Foltz et al., 2009; Shuaib et al., 2010). The exact role of NPM1 in CENP-A deposition remains somewhat unclear, but it is reasonable to suppose that its assembly activity for CENP-A may play a role in centromere activity given its presence in the CENP-A prenucleosomal complex and at the centromere (Foltz et al., 2009). In addition, three previously described chromatin remodeling complexes, FACT, CHD1, and RSF are also associated with the centromere and are important for the stable assembly of CENP-A containing chromatin (Foltz et al., 2006; Obuse et al., 2004; Okada et al., 2009; Perpelescu et al., 2009). Although, CHD1 appears to be dispensable in D. melanogaster for CID deposition (Podhraski et al., 2010). The activity of RSF1 appears to be restricted to mid G1, well after new CENP-A nucleosome deposition by HJURP, which immediately follows mitosis (Jansen et al., 2007; Perpelescu et al., 2009). The period of RSF1 recruitment may represent a remodeling event whereby the centromere is reorganized in preparation for the ensuing S-phase and mitosis.

Budding yeast and higher eukaryotes utilize Scm3/HJURP proteins for the common purpose of depositing CENP-A nucleosomes but use disparate mechanisms to determine the site of recruitment (Shivaraju et al., 2011). In the case of budding yeast, DNA sequence defines the site of CENP-A deposition, whereas in higher eukaryotes the site of new CENP-A incorporation is influenced by the location of the pre-existing CENP-A nucleosomes. Epigenetic inheritance requires the recruitment of HJURP 
through the activity of the Mis18 complex dictating the site of CENP-A nucleosome deposition. In conclusion, this work contributed to the understanding that nucleosomeassembled CENP-A is the epigenetic mark for centromere and kinetochore assembly in human cells. 


\section{$\underline{\text { Supplemental Figures }}$}

Supplemental Figure 2-1. NIH-3T3 LacO cell line also displays stable CENP-A deposition upon LacI-HJURP targeting

(A) NIH3T3-LacO-SceI-TetO cells were co-transfected with YFP-CENP-A and either LacI or LacI-HJURP. The indicated cells were treated with 5mM IPTG for 30 minutes prior to fixation. The LacO array is much larger than the endogenous centromeres. This large accumulation persists following the treatment with IPTG, abolishing LacI-LacO interactions. The LacO array does not overlap with endogenous centromeres as CENP-B staining, a marker specific for endogenous centromeres through binding of specific sequences in alpha satellite repeats, was not observed at the array. Scale bar $=5 \mu \mathrm{m}$. (B) Live-cell imaging was conducted using the NIH3T3 cell line that contains the LacO-SceITetO array. Cells co-transfected with YFP-CENP-A and LacI-HJURP or LacIHJURP $^{\text {Scm3 }}$ (bottom panel) were mock- treated or treated with 5mM IPTG 24 hours after transfection and followed by time-lapse microscopy. Upon the addition of IPTG, $80 \%$ of LacI-HJURP transfected cells retained YFP-CENP-A at the array even though LacIHJURP was undetectable. (C) YFP-CENP-A and LacI-HJURP intensities were measured, background subtracted and expressed in the graph as the percent of intensity versus $\mathrm{t}=0$. Error bars represent standard deviations. An average of $48 \%$ of YFP-CENP-A remained stably associated with the array 30 min following IPTG treatment, and a similar proportion $(53 \%)$ remained after 60 minutes. In some LacI-HJURP ${ }^{\mathrm{Scm} 3}$ cases, YFPCENP-A could still be seen at the array 24 hours after IPTG treatment. 
A

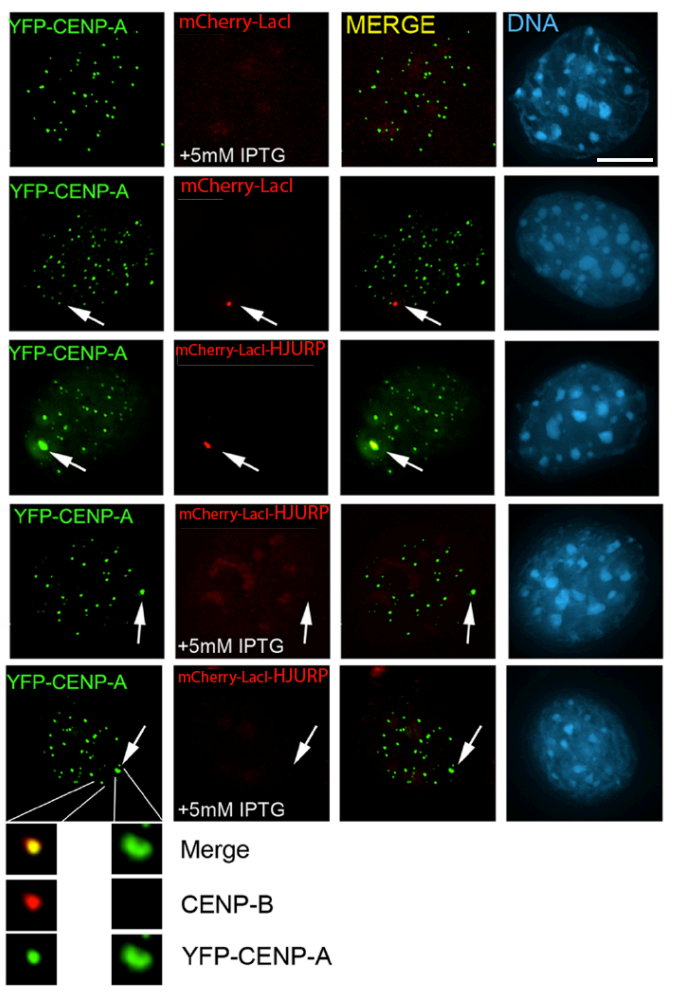

B

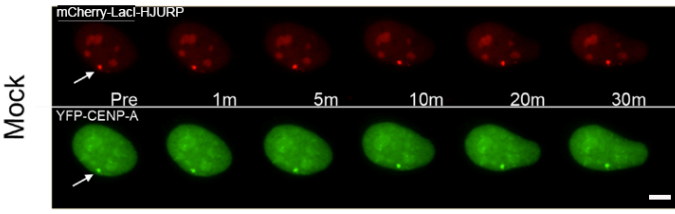

임
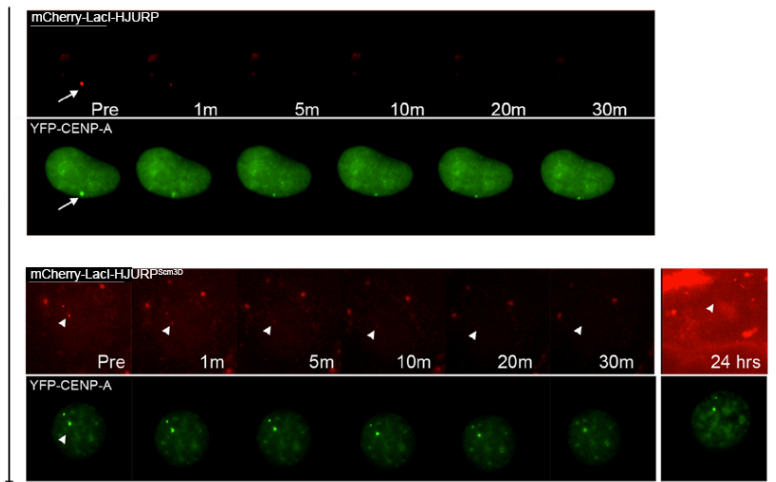

C

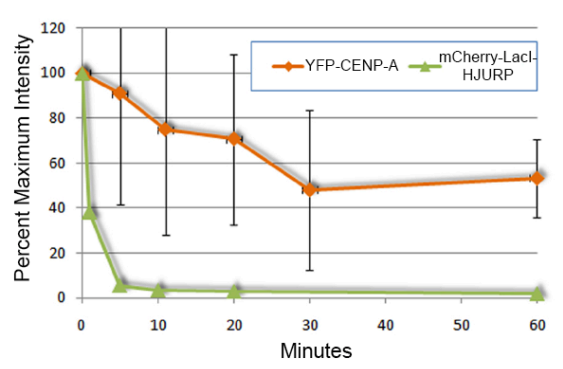


Supplemental Figure 2-2. CENP-A recruitment to the LacO increases over time and is stable for multiple generations after LacI-HJURP removal

(A) U2OS-LacO cells were transfected with mCherry-LacI-HJURP. Cells were preextracted and fixed at 24, 48, or 72 hours. Cells were stained with an antibody to endogenous CENP-A. Graph displays the percentage of mCherry-LacI-HJURP cells that were positive for CENP-A staining at each timepoint. $n=30$ cells per conditions, $\mathrm{N}=1$ replicate. (B) Representative images of U2OS-LacO cells that were co-transfected for 24 hours with GFP-TetR and mCherry-LacI-HJURP ${ }^{\mathrm{Scm} 3}$. After 24 hours of transfection, cells were treated with $15 \mathrm{mM}$ IPTG. 8 hours into IPTG treatment, cells were re-transfected with HA-CENP-A. Cells were left for an additional 48 hours in the presence of IPTG. GFP-TetR cells were then counted for the presence of HA-CENP-A. 6\% of GFP-TetR arrays were HA-CENP-A positive after 60 total hours in IPTG. N=1 replicate. 

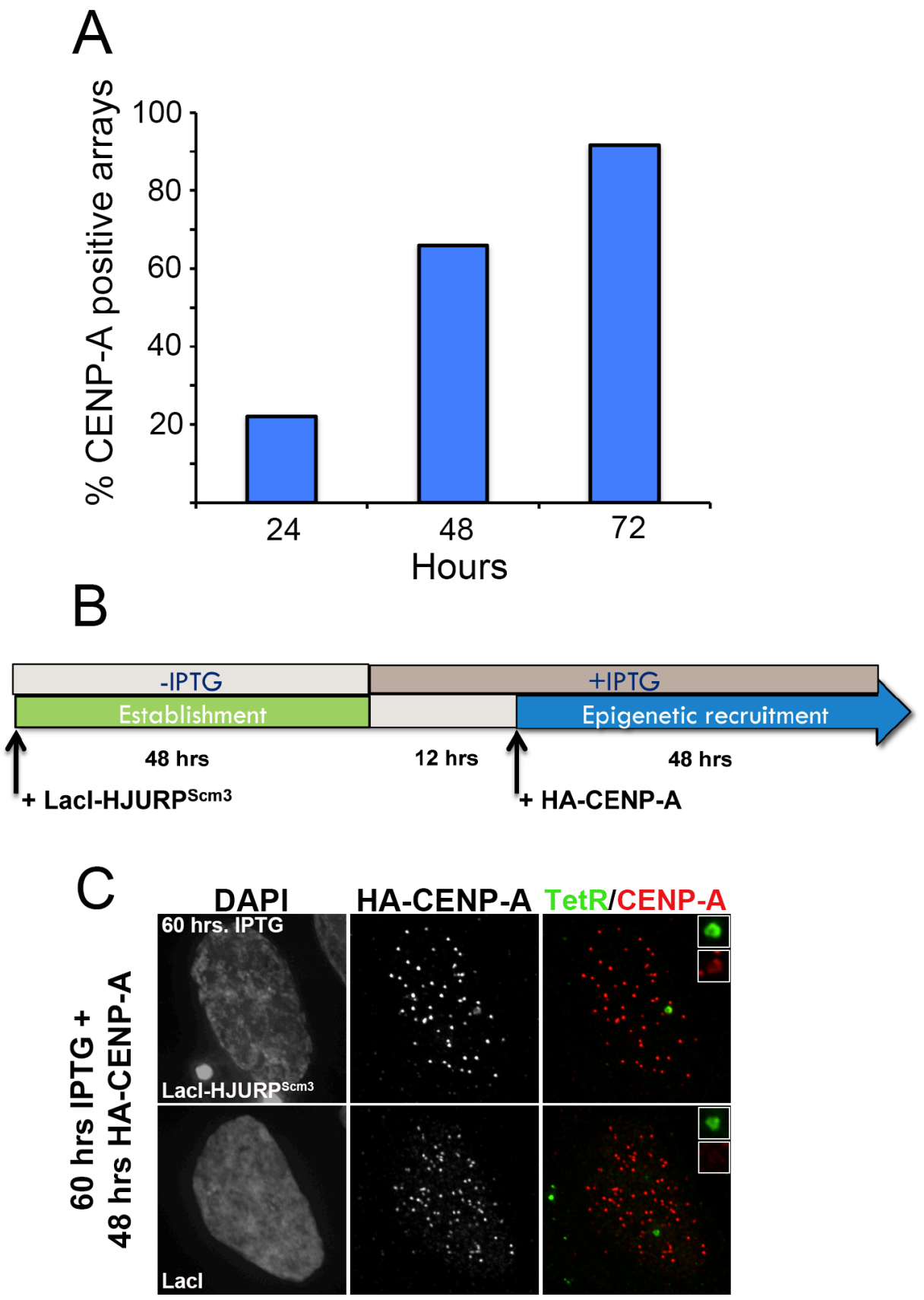
Supplemental Figure 2-3. Localization pattern of HJURP $P^{\mathrm{Scm} 3}$

(A) Schematic representations of HJURP fragments are shown (left panel). Representative images of centromeric localization are shown to the right with a centromeric marker (anti-CENP-T, red), the GFP-tagged HJURP fragments (green) and DNA (DAPI, blue). Insets display centromeres increased by 4X. HJURP (a.a. 1748(end)), HJURP ${ }^{\mathrm{Scm} 3}$ (a.a. 1-208), Scale bar $=5 \mu \mathrm{m}$. (B) GFP-HJURP ${ }^{\mathrm{Scm} 3}$ fails to localize to centromeres. Cells were transiently transfected with GFP-HJURP ${ }^{\mathrm{Scm} 3}$ and either fixed (B) or pre-extracted and then fixed. Scale bar $=5 \mu \mathrm{m}$. (C) GFP-HJURP ${ }^{\mathrm{Scm} 3}$ is clearly expressed in cells when cells are fixed without pre-extraction, as it is present in the cytoplasm and in the nucleus. GFP-HJURP $P^{\mathrm{Scm} 3}$ is not stably associated with centromeres, as no co-localization is observed in pre-extracted cells between GFPHJURP $^{\mathrm{Scm} 3}$ and the centromere marker CENP-T. 

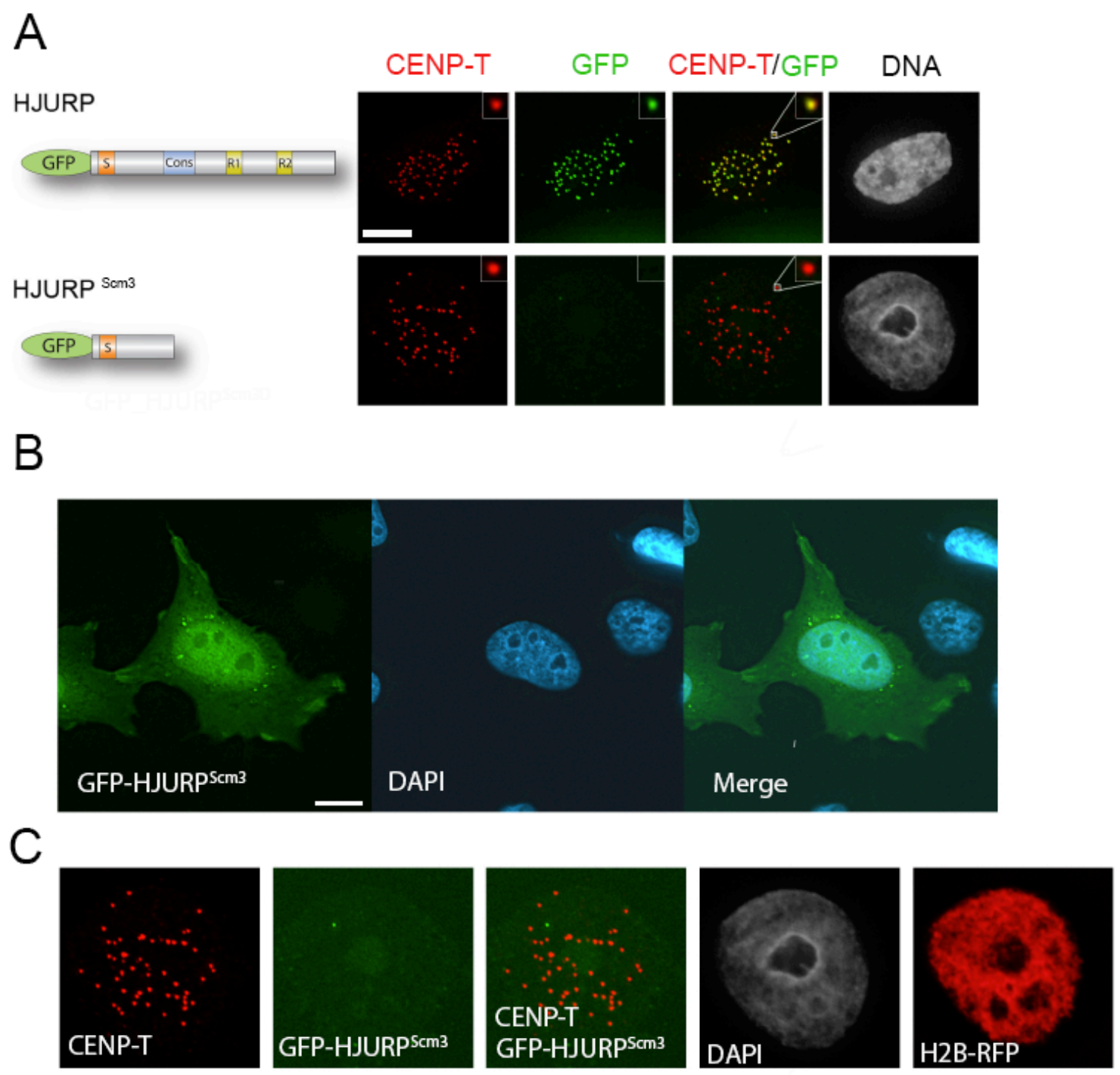

High-contrast 
Supplemental Figure 2-4. LacI-HJURP ${ }^{\mathrm{Scm} 3}$ recruits CCAN components most efficiently

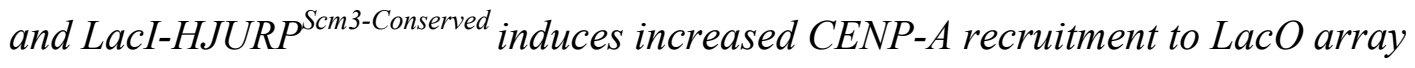

(A) Representative images of U2OS-LacO cells transfected for 48 hours with mCherryLacI-HJURP ${ }^{\text {Scm3 }}$, mCherry-LacI-HJURP ${ }^{\text {Scm3Conserved }}$, or mCherry-LacI-HJURP $P^{\text {FullLength }}$ plus GFP-CENP-C. Centromeres are stained with an antibody to CENP-A. Scale bar represents $5 \mu \mathrm{m}$. (B) Quantification of CENP-A positive LacI-HJURP arrays that also had GFP-CENP-C present. $\mathrm{n}=30$ cells per condition, $\mathrm{N}=1$ replicate. (C) Representative images of U2OS-LacO cells transfected for 48 hours with mCherry-LacI-HJURP ${ }^{\text {Scm3 }}$, mCherry-LacI-HJURP ${ }^{\text {Scm3Conserved }}$, or mCherry-LacI-HJURP ${ }^{\text {FullLength }}$ plus GFP-CENP-N. Centromeres are stained with an antibody to CENP-A. Scale bar represents $5 \mu \mathrm{m}$. (D) Quantification of CENP-A positive LacI-HJURP arrays that also had GFP-CENP-N present. $\mathrm{n}=30$ cells per condition, $\mathrm{N}=1$ replicate. (E) Quantification of the intensity of endogenous CENP-A recruited to the array with mCherry-LacI-HJURP ${ }^{\mathrm{Scm} 3}$, mCherryLacI-HJURP $P^{\text {Scm3Conserved }}$, or mCherry-LacI-HJURP ${ }^{\text {FullLength }}$ targeted to the array. Cells were either treated with $15 \mathrm{mM}$ IPTG for 1 hour prior to fixation or not to look at the stably assembled population of CENP-A. Intensity was measured using a constant ellipse size, $\mathrm{n}=20$ cells per condition. ${ }^{* *}$ Indicates $\mathrm{p}<0.0001$ by Kruskal-Wallis test followed by Dunn's multiple comparison test. 


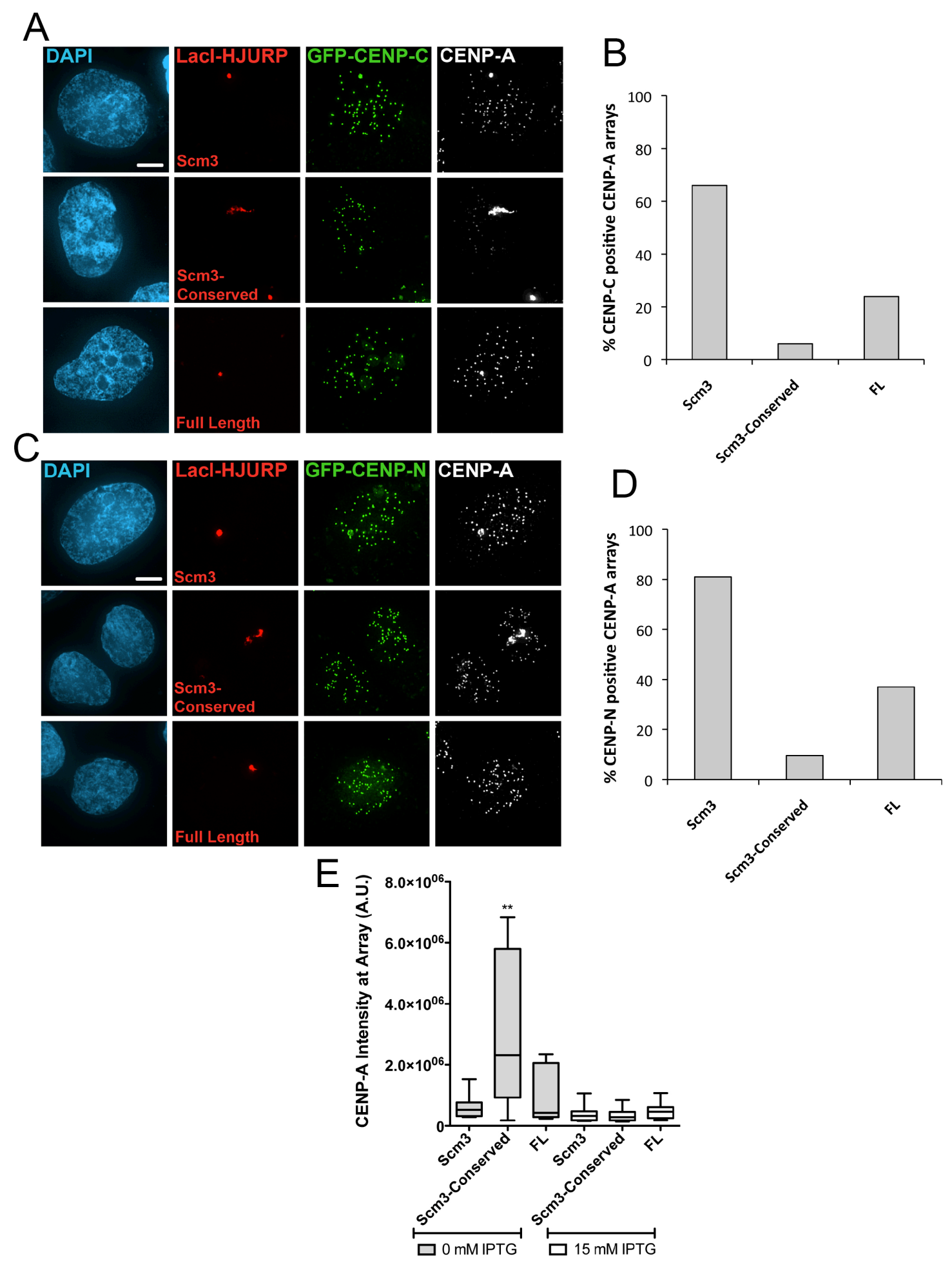


Supplemental Figure 2-5. Mad2 and Aurora B are recruited to LacI-HJURP $P^{S \mathrm{~cm} 3}$ assembled CENP-A arrays

(A) Representative images of U2OS-LacO cells transfected with either mCherry-LacI alone or mCherry-LacI-HJURP ${ }^{\mathrm{Scm} 3}$ for 48 hours. Cells were then arrested in nocodazole and prepared as mitotic spreads. Spreads were stained with an antibody to Aurora B. (B) Representative images of U2OS-LacO cells transfected with either mCherry-LacI alone or mCherry-LacI-HJURP ${ }^{\mathrm{Scm} 3}$ for 48 hours. Cells were then arrested in nocodazole and prepared as mitotic spreads. Spreads were stained with an antibody to Mad2. 
A
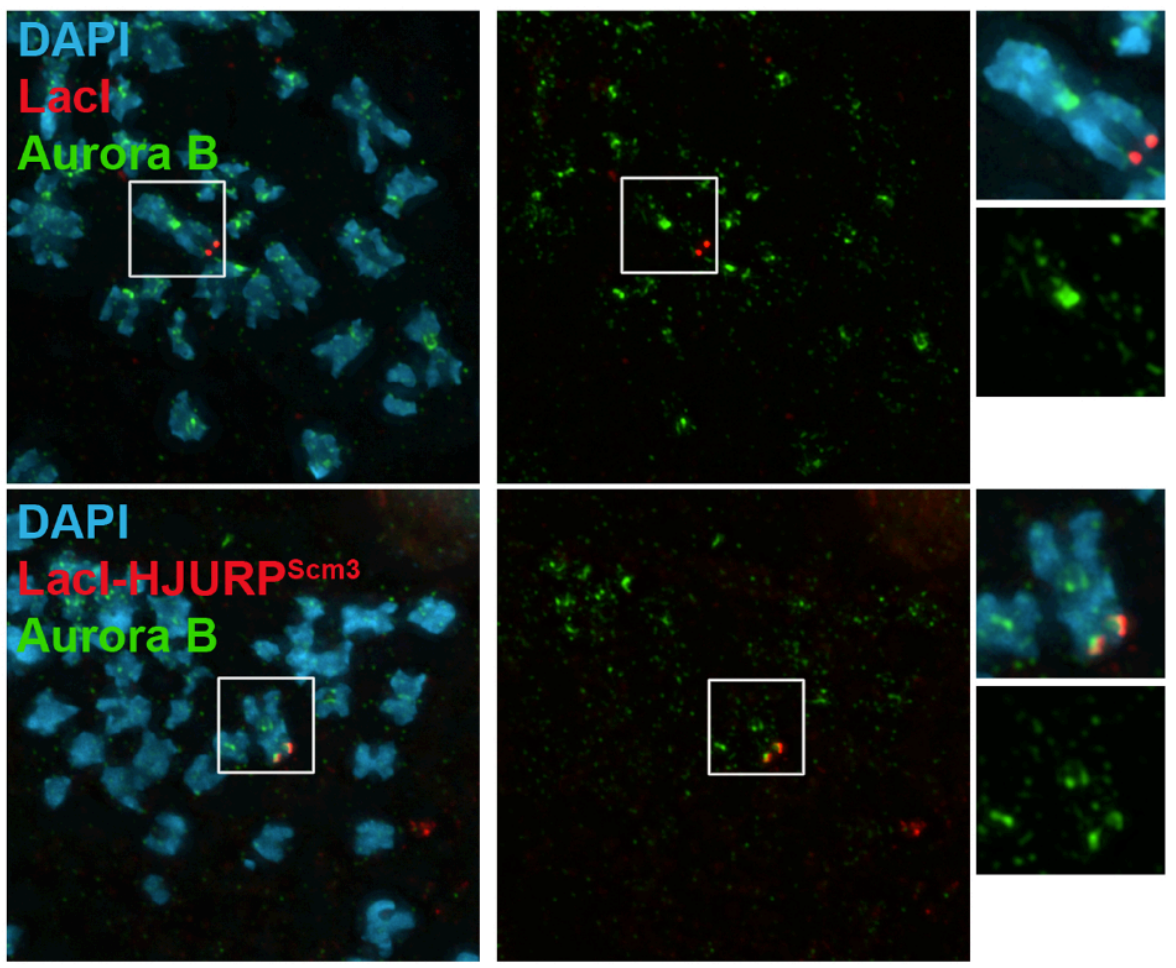

B
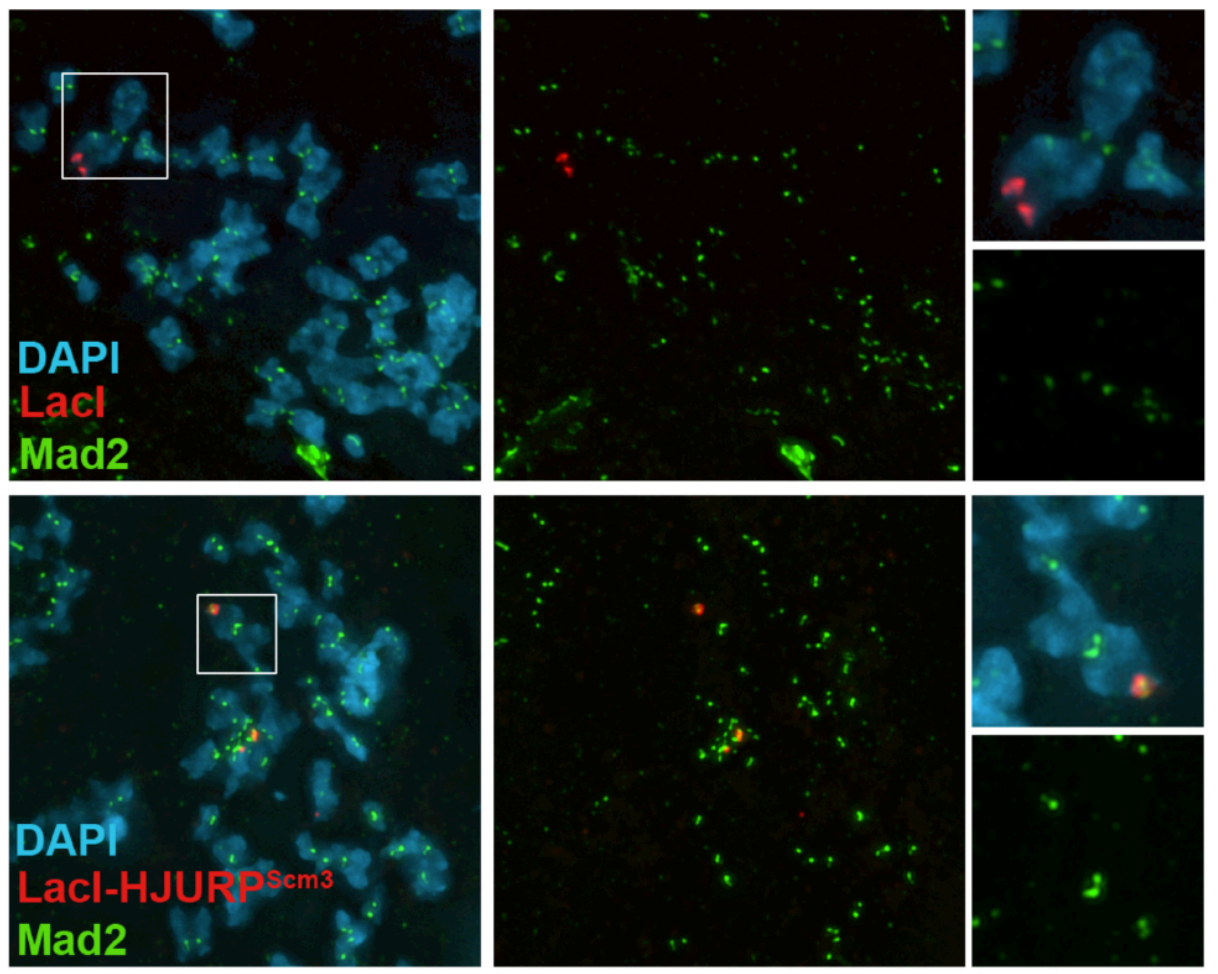
Supplemental Figure 2-6. Recombinant purifications of MBP-HJURP $P^{S c m 3}$ and line scans of band intensities from supercoiling assay

(A) Coomassie gel of recombinant MBP-HJURP ${ }^{\mathrm{Scm} 3}$, NPM1, and dNAP. (B) dNAP-1 is able to efficiently assembly histone H3.1 and CENP-A histone octamer onto a DNA template. (C) Line scans of band intensities from supercoiling assay in Figure 2-5 A, B. Boxes indicate the location of the maximally supercoiled plasmid population.

All data in Figure Suppl. Figure 2-6 were collected and assembled by Henning Kuich and Dan Foltz. 
A

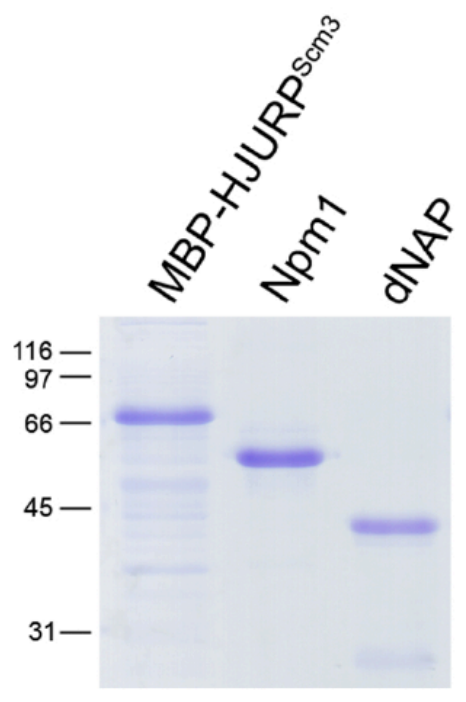

C

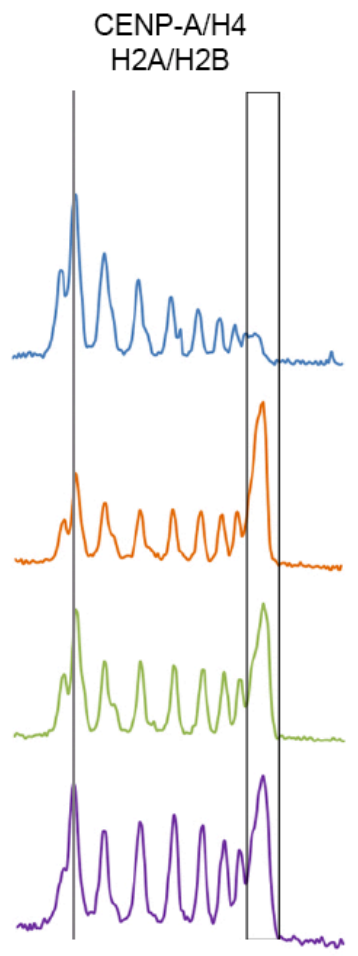

B

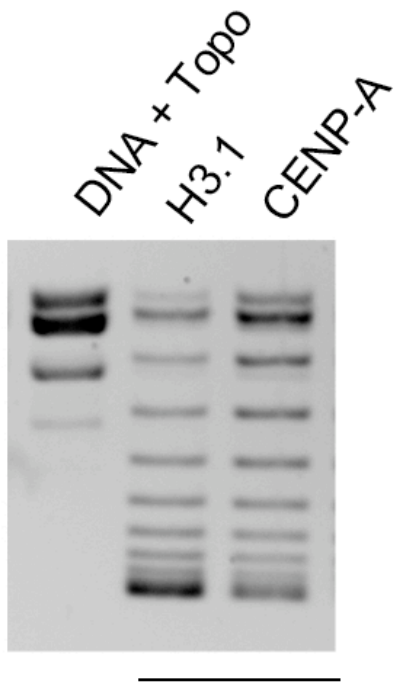

dNAP1
1X CENP-A/H4

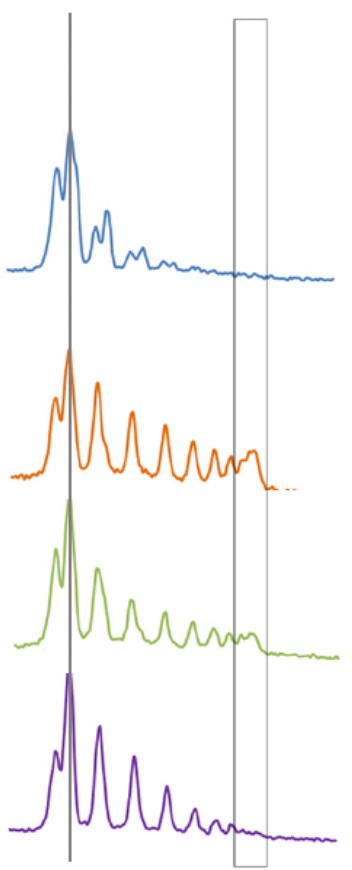

2X CENP-A/H4

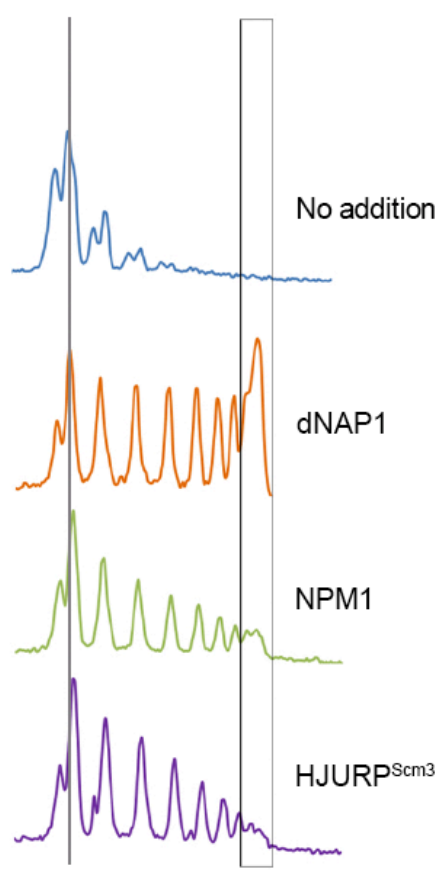


Supplemental Figure 2-7. GFP-Mis18a and GFP-HJURP stable lines display same localization as the endogenous proteins

Stable cell lines expressing LAP(GFP)-Mis18 $\alpha$ or LAP(GFP)-HJURP accumulate GFPMis $18 \alpha$ or GFP-HJURP to centromeres in a subset of cells. When cells are enriched for a G1 population using a single thymidine block and release, the proportion of cells where GFP-HJURP or GFP-Mis $18 \alpha$ are recruited to centromeres is significantly increased, consistent with the selective recruitment of the GFP tagged proteins during G1, similar to the endogenous proteins.

All data in Supplementary Figure 2-7 were collected and assembled by Madison Stellfox. 


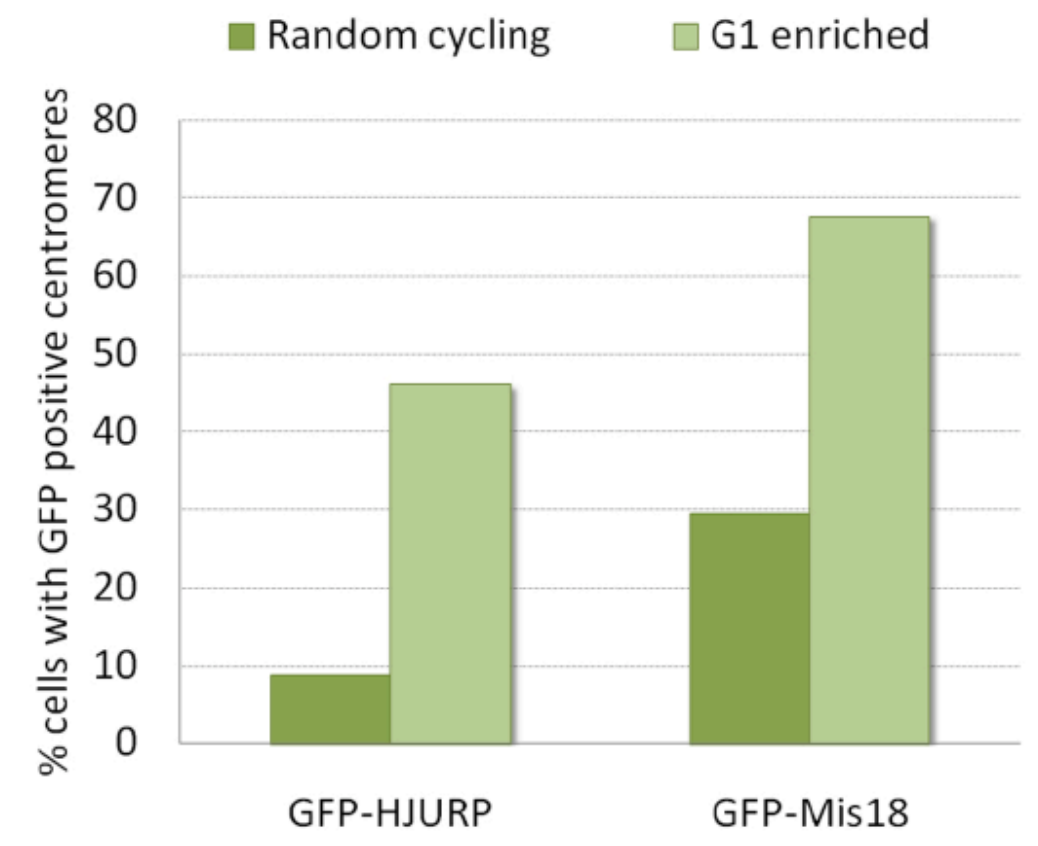


Supplemental Figure 2-8. GFP-HJURP ${ }^{S c m 3}-H 2 B$ causes CENP-A, CENP-C, and Hec1 mislocalization to chromosome arms

(A\&B) Representative images of U2OS-LacO cells transfected for 48 hours with either YFP-H2B in A or GFP-HJURPScm3-H2B in B. Cells were arrested in nocodazole overnight then prepared as mitotic spreads. Spreads were stained with antibodies to CENP-A, CENP-C, or Hec1. (C) Quantification of experiment in A and B. For each antibody analyzed, GFP-positive spreads were classified as displaying centromeric staining, arm staining, or both. $\mathrm{n} \geq 30$ spreads per antibody, per condition. (D\&F) Representative images of non-mitotic U2OS cells transfected for 48 hours with YFP-H2B or GFP-HJURPScm3-H2B. Cells were stained with an antibody to CENP-A in D or an antibody to CENP-C in F. (E) Quantification of D. Cells expressing GFP-HJURPScm3H2B (gray) or YFP-H2B (black) were classified as the percentage of GFP-positive cells having a visibly centromeric CENP-A staining pattern or only a nuclear CENP-A staining pattern. $n=30$ cells per condition. (G) Quantification of F. Cells expressing GFPHJURPScm3-H2B (gray) or YFP-H2B (black) were classified as the percentage of GFPpositive cells having visibly centromeric CENP-C. $n=30$ cells per condition. 


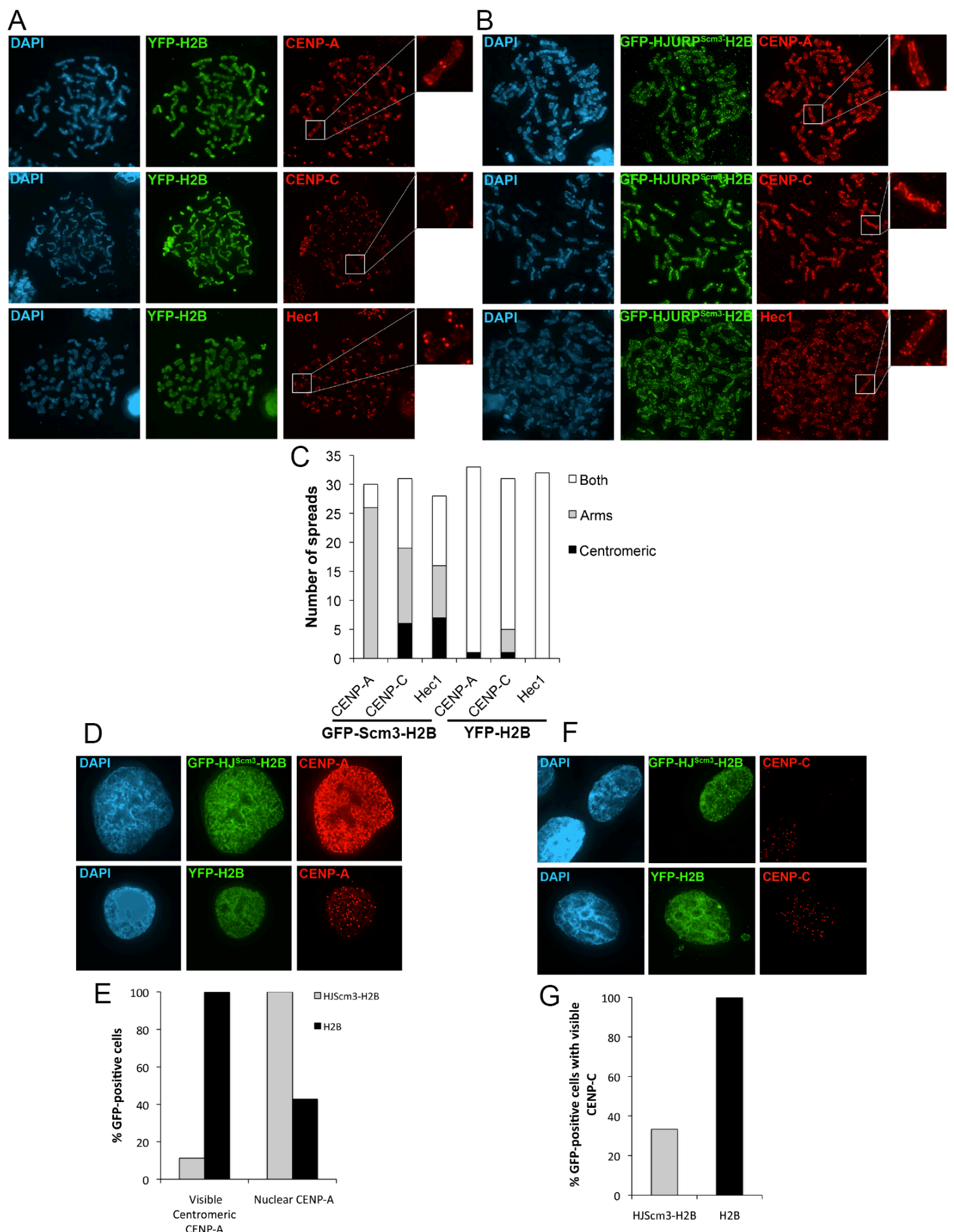




\section{Materials and Methods}

siRNA Treatment

HeLa cell lines expressing were plated at $8 \times 10^{5}$ cells in six-well plates. The next day, cells were transfected with $5 \mathrm{nM}$ Ambion Silencer Select siRNAs using RNAiMAX (Invitrogen). siRNA sequences were as follows: Mis18 $\alpha$ 5'GAagAuguCUUGAaAgCAUTT-3', Mis18BP1 $1^{\text {hsKNL2 }} \quad$ (C14orf106) 5'GGAUAUCCAAAUUAUCUCATT-3', 5'-CAAGUAUGAAGUUCGAUATT-3' .

The following day, $1 / 3$ of the plating volume of DMEM with $10 \%$ heat inactivated FBS was added. For western blot analysis, cells were harvested forty-eight hours after siRNA treatment with PBS +3 mM EDTA, counted and whole-cell lysates were made in SDSPAGE sample buffer. Lysates from $1 \times 10^{5}$ cells per lane were separated on $10 \%$ SDSPAGE gel and transferred to nitrocellulose. Blots were incubated in primary anti-GFP or anti-HJURP (Foltz et al., 2009) antibody overnight at $4^{\circ} \mathrm{C}$, and in secondary (Jackson Laboratories) for 1 hour at $4^{\circ} \mathrm{C}$. For U2OS-LacO cells, they were plated at $8 \times 10^{4}$ cells in 24-well plates onto poly-lysine coverslips. They were transfected after 24 hrs with $5 \mathrm{nM}$ Ambion Silencer Select siRNAs (same sequence as above for Mis18BP1 ${ }^{\text {hsKNL2 }}$ siRNA). After 24 hrs in siRNA, cells were transiently transfected with mCherry-LacI-HJURP ${ }^{\text {Scm3 }}$ and GFP-TetR using Effectene (Qiagen) into the media still containing the siRNA. Following 8 hrs of transfection, the media was removed, and cells were re-treated with siRNA. Cells were then incubated for $48 \mathrm{hrs}$ and treated with $15 \mathrm{mM}$ IPTG ((Sigma Cat\# I675) for $1 \mathrm{hr}$ prior to pre-extraction, fixation, and staining for endogenous CENP-A as described below. 


\section{Cell culture, transfections and immunocytochemsitry}

HeLa or U2OS-LacO-TRE cells were plated to poly-lysine coated coverslips at $1.6 \times 10^{5}$ cells per well in 6 well or $0.8 \times 10^{5}$ cells per well in 24 -well plates, respectively. Cells were then transfected 24 hours later with 0.2-0.25 ug plasmid DNA (24-well plate) or 1ug (6-well plate) using GeneJuice (EMD Chemicals, Cat\# 70967) or Effectene (Qiagen, Cat\# 301425). NIH3T3 cells were transfected with Lipofectamine ${ }^{\mathrm{TM}} 2000$ (Invitrogen, 11668-027). Live-cell imaging was conducted in the NIH3T3-LacO cell line at $37^{\circ} \mathrm{C}$ in Leibovitz L15 media including 10\% FBS following $48 \mathrm{hrs}$ of transfection. Single plane images were collected at 1 minute intervals on a Deltavision Microscope equipped with a Weatherstation environmental chamber maintained at $37^{\circ} \mathrm{C}$.

HeLa cells were pre-extracted with $0.1 \%$ Triton-X in PBS for 3 minutes, fixed with $4 \%$ formaldehyde in PBS for 10 minutes and then quenched by addition of $100 \mathrm{mM}$ Tris, $\mathrm{pH} 7.5$ for another 10 minutes at room temperature. HeLa cells were blocked in $2 \%$ FBS, $2 \% \mathrm{BSA}$ in $0.1 \%$ Trition-PBS. $\mathrm{U}_{2} \mathrm{OS}^{\mathrm{LacO} / \mathrm{TETr}}$ cells were treated with 0 or $10 \mathrm{mM}$ IPTG for $1 \mathrm{hr}$ in DMEM GlutaMax media prior to fixation then pre-extracted in PHEM buffer using $0.1 \%$ Triton-X-100 for 3 min and finally fixed with $4 \%$ paraformaldehyde in PBS for 10 minutes at room temperature. The cells were quenched for $5 \mathrm{~min}$ in $100 \mathrm{mM}$ Tris- $\mathrm{HCl}$ followed by a 1 hour block in 2\% FBS, 2\%BSA, 0.1\% Triton-X-100 in PBS. Centromeres were visualized with a rabbit polyclonal anti-CENP-T antibody or monoclonal CENP-A at 1:1000 dilution (Abcam, ab13939) and DNA was stained with 4',6-diamidino-2-phenylindole (DAPI, $0.2 \mathrm{mg} / \mathrm{ml}$ ). Donkey-anti-rabbit Cy5-conjugated (Jackson Laboratories, \#111175003) secondary antibodies were used for detection and 
coverslips were mounted with Prolong (Invitrogen). GFP-TetR marker detection was enhanced in mitotic spreads and the Mis18BP $1^{\mathrm{h} S K N L 2}$ siRNA experiment by staining with a rabbit anti-GFP antibody (1:500) and donkey-anti-rabbit Fitc-conjugated secondary antibody (Jackson Labs, \# 711095152).

All micrograph images were collected using a 100x oil-immersion Olympus objective lens (numerical aperture $=1.40$ ) on a DeltaVision deconvolution microscope using a Photometrics CoolSNAP $\mathrm{HQ}^{2}$ camera. Acquisition software used was SoftWoRX from Applied Precision. Images were deconvolved and presented as stacked images. Images within cell lines treated with different siRNAs were collected with identical exposure times and scaled equally. Intensities in live-cell and fixed images were analyzed using MetaMorph 7.7. mCherry and YFP signals in live-cell images were subjected to local background subtraction. For determining siRNA knockdown, nuclei with all CENP-T foci co-localized with GFP-HJURP were scored as GFP-HJURP positive. Cells that had GFP-Mis $18 \alpha$ at all centromeres with a maximum intensity above 7600 A.U.s were scored as GFP-Mis $18 \alpha$ positive. To assess CENP-A intensity at LacO/TRE array images of mCherry-LacI-HJURP or mCherry-LacI-HJURP ${ }^{\mathrm{Scm} 3}$ transfected and fixed/immunostained U2OS-LacO cells +/- IPTG treatment 48 hours after transfection were taken under identical exposure conditions and analyzed for CENP-A intensity at the array using MetaMorph 7.7. CENP-A signal was subjected to local background subtraction. Intensity measurements were made per a set area and the integrated intensities over this area for each group of cells were graphed. 


\section{Mitotic Chromosome Spreads}

U2OS-LacO cells were arrested overnight in $0.1 \mathrm{ug} / \mathrm{mL}$ Nocodazole in DMEM GlutaMAX media 32 hours after transfection. Mitotic cells were harvested using a transfer pipette to blow cells off the plate. Cells were spun down, washed in 1xPBS, and resuspended at $1 \times 10^{6}$ cells $/ \mathrm{mL}$ in a hypotonic solution (20 mM Hepes (pH 7.0), $1 \mathrm{mM}$ $\mathrm{MgCl}_{2}, 0.2 \mathrm{mM} \mathrm{CaCl} 2,20 \mathrm{mM} \mathrm{KCl}, \mathrm{LPC}$, and $0.5 \mathrm{ug} / \mathrm{mL}$ nocodazole/colcemid). After a 10 min incubation in the hypotonic solution, cells were spun onto glass slides using a cytospin $(30,000$ cells/slide), immediately hydrated with 1xPBS, and then fixed and immunostained as described above. The anti-mouse NDC80 antibody was used at a 1:500 dilution (GeneTex, GTX70268).

\section{Monastrol arrest}

For U2OS-LacO cells transfected for 48 hours with mCherry-LacI-HJURP ${ }^{\mathrm{Scm} 3}$ or mCherry-LacI, as described above, were arrested overnight in $50 \mathrm{uM}$ Monastrol in DMEM GlutaMAX media. Mitotic cells were harvested by mitotic blow-off, spun down, rinsed with $1 \times$ PBS, then resuspended at $1 \times 10^{6}$ cells $/ \mathrm{mL}$. Using a cytospin, 30,000 cells/slide were spun onto a glass slide. Cells were immunostained with an anti-rabbit CENP-T antibody (described above). For the quantification, 26 transfected, Monastrolarrested cells were imaged per condition (LacI or LacI-HJURP ${ }^{\mathrm{Scm} 3}$ ). An ellipsoid was drawn to encompass the DNA, and the position of each centromere in each cell was measured relative to the center of this ellipsoid using ImageJ. The black circle represents the average centromere position over all the cells analyzed. The next gray circle represents one standard deviation from this average. The blue triangles (LacI) and 
red diamonds (LacI-HJURP ${ }^{\mathrm{Scm} 3}$ ) represent the array positions relative to the center of the ellipsoid in each of the 26 cells measured.

\section{Cold stable microtubules}

Cells were transfected as indicated above with either mCherry-LacI or mCherry-LacIHJURP $^{\mathrm{Scm} 3}$ and synchronized using a double thymidine block. Following the second block, cells were released until they were entering mitosis. Cells were then placed on ice and treated with ice-cold media for 10 minutes. Cells were then co-fixed $(2 \%$ paraformaldehyde, $0.5 \%$ Triton-X-100 in 1x Phem buffer) and immunostained for CENPT and tubulin (using a FITC-conjugated tubulin antibody).

\section{Recombinant protein purification}

Recombinant proteins were expressed in the Rosetta (DE3) pLysS bacterial strain. Bacteria expressing His-tagged dNAP1 were sonicated in Lysis Buffer containing $50 \mathrm{mM}$ sodium phosphate, $\mathrm{pH} 7.5,500 \mathrm{mM} \mathrm{NaCl}, 10 \mathrm{mM}$ imidazole, $10 \%$ glycerol, $10 \mathrm{mM}$ bglycerophosphate, $0.2 \mathrm{mM}$ PMSF, $0.5 \mathrm{mM}$ benzamidine and purified by Ni-NTA affinity chromatography. dNAP1 was further purified by size exclusion chromotagraphy on a Superose 6 column in buffer containing $10 \mathrm{mM}$ Hepes/KOH pH 7.6, $10 \mathrm{mM} \mathrm{KCl,} 0.1$ mM EDTA, 10\% glycerol, 0.01\% NP-40, 10 mM b-glycerophosphate, $0.2 \mathrm{mM}$ PMSF, 1 mM DTT followed by anion exchange chromatography on an UnoQ (BioRad) column. The Topoisomerase I catalytic domain (TopoI) (Shaiu and Hsieh, 1998) was purified as described previously (Fyodorov and Kadonaga, 2003). Bacterial lysates were sonicated in $50 \mathrm{mM}$ sodium phosphate, $\mathrm{pH} 7.0,0.5 \mathrm{M} \mathrm{NaCl}, 15 \%(\mathrm{v} / \mathrm{v})$ glycerol, $0.1 \%(\mathrm{v} / \mathrm{v}) \mathrm{NP}-40$ and purified by Ni-NTA affinity chromatography. A codon-biased human CENP-A was 
co-expressed with histone H4 from a bi-cistronic vector (Black et al., 2004). The CENPA/histone $\mathrm{H} 4$ heterotetramer was purified by hydroxyapatite chromatography followed by cation exchange chromatography. Canonical histones were individually expressed and purified by size exclusion chromatography in $7 \mathrm{M}$ guanidinium $\mathrm{HCl}, 20 \mathrm{mM}$ Tris-HC1, pH 7.5, $10 \mathrm{mM}$ DTT on a Sephacryl S200 column followed by cation exchange chromotargraphy in $7 \mathrm{M}$ deionized urea, $20 \mathrm{mM}$ sodium acetate, $\mathrm{pH} 5.2,5 \mathrm{mM} 2$ mercaptoethanol, $1 \mathrm{mM} \mathrm{Na-EDTA}$ as described previously (Luger et al., 1999). HisNPM bacterial pellets were resuspended in buffer containing $50 \mathrm{mM}$ Na-phosphate, $\mathrm{pH}$ $8.0,300 \mathrm{mM} \mathrm{NaCl}, 10 \mathrm{mM}$ Imidazole, $10 \mathrm{mM}$ beta-glycerophosphate, $1.5 \mathrm{mM} \mathrm{MgCl}_{2}$, $0.5 \mathrm{mM}$ PMSF and supplemented with $1 \mathrm{mg} / \mathrm{ml}$ lysozyme. The mixture was incubated on ice for 30 minutes and then lysed by using a French press. 1\% Triton-X was added and the solution was centrifuged at $26.9 \mathrm{Kxg}$ for 10 minutes at $4^{\circ} \mathrm{C}$. The supernatant was incubated with Ni-NTA agarose beads (Qiagen) for 1.5 hours at $4^{\circ} \mathrm{C}$. Beads were washed 3 times with buffer $(50 \mathrm{mM}$ Na-phosphate buffer, $\mathrm{pH} 8.0,300 \mathrm{mM} \mathrm{NaCl}, 20 \mathrm{mM}$ Imidazole, $10 \mathrm{mM}$ beta-glycerophosphate, $1.5 \mathrm{mM} \mathrm{MgCl}_{2}, 0.5 \mathrm{mM} \mathrm{PMSF}$ ), and finally eluted in elution buffer (50 mM Na-phosphate buffer, $\mathrm{pH} 8.0,150 \mathrm{mM} \mathrm{NaCl}, 250 \mathrm{mM}$ Imidazole, $10 \mathrm{mM}$ beta-glycerophosphate, $1.5 \mathrm{mM} \mathrm{MgCl}_{2}, 1 \mathrm{mM}$ DTT). MBP-HJURP ${ }^{\mathrm{Scm} 3}$ bacterial pellets were resuspended in lysis buffer $(25 \mathrm{mM}$ Tris-Cl, $\mathrm{pH} 7.2,200 \mathrm{mM} \mathrm{NaCl}$, $20 \mathrm{mM} \mathrm{MgCl}_{2}, 10 \%$ Glycerol, $5 \mathrm{mM}$ beta-mercaptoethanol, $10 \mathrm{mM}$ beta-glycerophosphate, 0.2mM PMSF, $1 \mathrm{mM}$ Benzamidine, LPC), stirred on ice for 30 minutes, and sonicated until no longer viscous. The sample was then centrifuged at $26.9 \mathrm{Kxg}$ for 15 minutes at $4^{\circ} \mathrm{C}$, the supernatant of was incubated with amylose resin (NEB) for 1 hour at $4^{\circ} \mathrm{C}$. After washing the beads in 10 bead volumes of washing buffer $(25 \mathrm{mM}$ Tris-Cl, $\mathrm{pH} 7.2$, 
$200 \mathrm{mM} \mathrm{NaCl}, 20 \mathrm{mM} \mathrm{MgCl} 2,10 \%$ Glycerol, $5 \mathrm{mM}$ beta-mercaptoethanol, $10 \mathrm{mM}$ betaglycerophosphate, $0.5 \mathrm{mM}$ PMSF, $1 \mathrm{mM}$ Benzamidine, $\mathrm{LCP}$ ), the protein was eluted from beads in elution buffer $\left(25 \mathrm{mM}\right.$ Tris-Cl, $\mathrm{pH} 7.2,200 \mathrm{mM} \mathrm{NaCl}, 20 \mathrm{mM} \mathrm{MgCl}_{2}, 10 \%$ Glycerol, $5 \mathrm{mM}$ beta-mercaptoethanol, $10 \mathrm{mM}$ beta-glycerophosphate, $10 \mathrm{mM}$ maltose).

\section{Nucleosome Assembly}

In buffer 1 (20mM HEPES, pH7.9, 1mM EDTA, 10\% Glycerol, 150mM NaCl, 0.1 $\mathrm{mg} / \mathrm{ml} \mathrm{BSA}$ ), purified histones (octamer, $\mathrm{H} 3 / \mathrm{H} 4$ tetramer, $\mathrm{H} 2 \mathrm{~A} / \mathrm{H} 2 \mathrm{~B}$ dimers) and purified chaperone were combined in a total volume of $20 \mu \mathrm{l}$ and incubated at $37^{\circ} \mathrm{C}$ for $15-30$ minutes (chaperone/histone mix). In a separate reaction, $0.25 \mu \mathrm{g}$ pUC19 plasmid DNA was added to buffer 2 (20mM HEPES, pH7.9, 1mM EDTA, 10\% Glycerol, 150mM $\mathrm{NaCl}$ ), containing purified Topoisomerase I. This mixture was incubated for 20 minutes at $37^{\circ} \mathrm{C}$ and then combined with the chaperone/histone mix, followed by an incubation period of 3 hours at $37^{\circ} \mathrm{C}$. Where indicated, $3 \mathrm{mM} \mathrm{CaCl}_{2}$ was added and reactions were treated with 3 units MNase (S1 nuclease, Roche) for 10 minutes at $37^{\circ} \mathrm{C}$. The assembly reaction was stopped by addition of EDTA to a final concentration of $50 \mathrm{mM} .100 \mu \mathrm{l}$ of glycogen stop buffer (20mM EDTA, pH8.0, 200mM NaCl, $1 \%$ SDS, $0.25 \mathrm{mg} / \mathrm{ml}$ glycogen) and $10 \mu \mathrm{g}$ proteinase $\mathrm{K}$ were added, and the protein digestion carried out at $37^{\circ} \mathrm{C}$ for $30-60$ minutes. Recovery of DNA was achieved by phenol-chloroform extraction and ethanol precipitation. Topoisomers were separated in $0.8 \%$ TBE agarose gels with or without $4.6 \mu \mathrm{M}$ chloroquine and subsequently stained with ethidium bromide. 


\section{$\underline{\text { Acknowledgements }}$}

Thanks to Alexandra Lusser, Sepideh Khorasanizadeh, Tom Misteli, Andrew Holland, Don Cleveland and Susan Janicki for reagents. Also thanks to Jonathan Gaba and Agnes Sabat for technical assistance and Todd Stukenberg and Dan Burke for comments on this manuscript. DRF was supported by the American Cancer Society. MCB was supported by Cell and Molecular Biology training grant (NIH \# T32 GM008136) and a UVA Farrow Fellowship. B.E.B. is supported by a research grant (NIH GM82989), a Career Award in the Biomedical Sciences from the Burroughs Wellcome Fund, and a Rita Allen Foundation Scholar Award. E.A.B. has been supported by the Penn Structural Biology Training Grant (NIH GM08275) and a predoctoral fellowship from the American Heart Association. The authors claim no commercial affiliations or conflicts of interest. 


\section{CHAPTER III: HJURP INTERACTION WITH SINE28 RNA VIA ITS VERTEBRATE CONSERVED DOMAIN IS REQUIRED FOR CENP-A DEPOSITION}

This chapter covers work done in collaboration with Rachel O'Neill's lab at the University of Connecticut. The manuscript is currently being assembled for initial submission. 


\section{$\underline{\text { Abstract }}$}

In this study we identify SINE28, a short, single-stranded RNA that is enriched at the centromere throughout the cell cycle. In the cell, this SINE28 RNA is bound to both CENP-A and the CENP-A chaperone HJURP. The CENP-A chaperone HJURP contains a conserved domain from amino acids $208-350$ that we show is required for SINE28 RNA binding. We additionally show that this domain is required for HJURP to localize to the nucleolus. HJURP that lacks this conserved domain is not capable of rescuing CENP-A deposition when endogenous HJURP is depleted from the cell. While HJURP lacking the conserved domain is able to localize to centromeres and bind to CENP-A in a comparable manner to full length HJURP, it is deficient at loading new CENP-A into centromeric chromatin arguing for an important role for RNA interaction in the CENP-A nucleosome assembly pathway. These data contribute SINE28 RNA as another player in the CENP-A deposition pathway in human cells. 


\section{$\underline{\text { Introduction }}$}

The centromere-specific histone H3 variant, CENP-A, possesses a specific chaperone from yeast to humans. Scm3 in yeast, CAL1 in flies, and HJURP vertebrates, these CENP-A specific chaperones contain one highly conserved region, the Scm3 domain, where the chaperones interact with CENP-A (Sanchez-Pulido et al., 2009; Shuaib et al., 2010). In humans, the CENP-A specific chaperone and nucleosome assembly factor is HJURP (ㅂolliday junction recognition protein) (Dunleavy et al., 2009; Foltz et al., 2009). The CENP-A chaperones vary in size, with the HJURP vertebrate version being much larger than the yeast $\mathrm{Scm} 3$ version $(89 \mathrm{kDa}$ versus about $25 \mathrm{kDa}$ for the yeast Scm3 protein) (Sanchez-Pulido et al., 2009). The role for the extended Cterminus of the vertebrate HJURP proteins is partially understood. There are two Cterminal repetitive regions in HJURP that are responsible for centromere targeting and dimerization of the protein (Zasadzinska, et al 2013). An additional domain that is

present in vertebrate HJURP but is absent from lower multi-cellular organisms is a central conserved region. It is present from frogs to humans and as such, is termed the vertebrate conserved region. It encompasses amino acids 228-304 in the human HJURP protein (Sanchez-Pulido et al., 2009). The functional relevance of this vertebrate conserved region was unknown prior to this study. Here, we will develop a role for the vertebrate conserved domain in RNA binding and demonstrate the importance of this RNA binding for CENP-A deposition.

Studies on the role of RNA in CENP-A deposition have mainly focused on characterizing the part of centromeric repeat transcription in CENP-A deposition. ChIP 
studies from maize, mice, and humans found association of CENP-A/CENH3 with alphasatellite RNA (Ferri et al., 2009; Topp et al., 2004; Wong et al., 2007). In budding yeast, transcription factor binding at centromeres is required for centromere function. Ectopically driving RNA Pol II activity over the CEN region is sufficient to rescue the high chromosome missegregation rates induced by transcription factor binding loss (Ohkuni and Kitagawa, 2011). Studies in human cells found that RNA Pol II inhibition during mitosis induces a reduction in the number of alpha-satellite transcripts detected at the centromere as well as a slight increase in lagging chromosomes during anaphase (Chan et al., 2012). Alpha-satellite transcripts were also shown to be present in CENP-C purifications, indicating that the alpha-satellite repeats at human centromeres are being actively transcribed (Wong et al., 2007). This indicates some level of transcription is required for proper centromere and kinetochore function in human cells during mitosis. Additionally, transcriptionally active LINE retrotransposons within CENP-A regions on a human neocentromere are required for successful CENP-A binding and incorporation (Chueh et al., 2009). Studies in marsupials have also identified a novel class of small RNA that is composed of satellite and retroviral sequences and emanates from the centromeres in these cells (Carone et al., 2009). Artificially inhibiting transcription by targeting LSD1 to a human artificial chromosome centromere caused a loss of HJURP recruitment and subsequent CENP-A loss (Bergmann et al., 2011). Complementary to this, driving high transcription over a HAC centromere also caused kinetochore inactivation and CENP-A loss (Bergmann et al., 2012). These studies emphasize that transcription, and specifically the amount of transcription, is a key factor for centromere stability and CENP-A deposition. 
Additional studies have discovered a structural role for RNA in proper CCAN and kinetochore recruitment. CPC members Aurora B, Survivin, and INCENP interact with RNA and require RNA for their centromeric localization (Ferri et al., 2009; Wong et al., 2007). Similarly, CENP-C requires RNA for its centromeric recruitment and for its DNA binding activity (Chan et al., 2012; Du et al., 2010; Wong et al., 2007). Treating mammalian cells with RNase results in a reduction of CENP-C, CENP-W, and even CENP-A at centromeres (Carone et al., 2009; Chun et al., 2011; Wong et al., 2007). However, many of these studies did not quantify the amount of each of these proteins that was lost upon RNase treatment, so it is not well understood if RNase causes a partial or complete loss of these proteins. So in addition to transcription and the RNA that results from that being important for CENP-A deposition, RNA also plays a crucial structural role at the centromere.

Lastly, nucleoli and centromeres have a documented relationship in higher eukaryotes. Nucleoli are well known for being the sites of ribosomal RNA synthesis and as such, are concentrated with RNA. In G1, S, and G2 nuclei, centromeres cluster around nucleoli on average $60 \%$ of the time (Foltz et al., 2009). This is not a well-understood phenomenon but may play a role in centromere assembly and maintenance. There is recent evidence in Drosophila that centromere nucleolar clustering mediates centromere pairing in meiosis I (Unhavaithaya and Orr-Weaver, 2013). Many of the proteins that require RNA for their centromeric recruitment, also localize to nucleoli. HJURP, CENPC, CENP-W, INCENP, Npm1, and the Mis18 complex all enrich at nucleoli in human cells (Chun et al., 2011; Dunleavy et al., 2009; Ferri et al., 2009; Foltz et al., 2009; Wong et al., 2007). CENP-W interacts with Npm1, and this interaction is RNase A sensitive 
(Chun et al., 2011). All these data suggest nucleoli may play a role in centromere and kinetochore assembly and support the notion that RNA serves an important functional role in these processes.

Concurrent with our work investigating the function of the vertebrate conserved domain of HJURP in human cells, Rachel O'Neill's lab discovered a centromere-specific short, single-stranded RNA, SINE28, which is present at human centromeres throughout the cell cycle (Introduction Figure 3-1 B). CENP-A and HJURP RNA immunoprecipitation experiments revealed that HJURP and CENP-A bind distinct regions of SINE28 based on which nucleotides purified most commonly with each protein (Introduction Figure 3-1). SINE28 was the most abundant RNA in both the CENP-A and HJURP RNA immunoprecipitation datasets compiled by the O'Neill lab. We had preliminary evidence that the vertebrate conserved domain of HJURP was a nucleic acid binding domain, and so we started a collaboration to study if the vertebrate conserved domain of HJURP was responsible for SINE28 interaction.

SINE28 (Short Interspersed Element derived from 28S ribosomal subunit) is a 151 nucleotide RNA polymerase III derived small single stranded RNA. This is unique from alpha-satellite transcripts, which are transcribed by RNA polymerase II (Chan et al., 2012; Wong et al., 2007). Short interspersed nuclear elements (SINE's) are transposable elements that are abundantly found in eukaryotic genomes. They are derived from reverse transcription of cellular RNA's and are then integrated into the genome. They are nonautonomous transposable elements and require another element to retrotranspose (Eickbush, 1992). SINE's are identifiable because they have a 5' sequence that is derived from a progenitor RNA, such as the 5 or $28 \mathrm{~S}$ rRNA's, and they contain an RNA 
polymerase III promoter sequence. They then have an intervening sequence followed by a 3' tail with which they are recognized by the autonomous element they use to retrotranspose (Longo, et al. Under review 2014). SINE28 was derived from the 3' end of the large ribosomal subunit rDNA (LSU/28S ribosomal subunit). It has 27 mapped locations throughout the genome in human cells, and it uses LINE1 transactivation to mobilize. The SINE28 sequence is present and highly conserved in all of the available sequenced mammalian genomes, and this is consistent with it having a valuable function in the cell (Longo, et al. Under review 2014). Interestingly, SINE28 is the most abundant RNA found in both the CENP-A and HJURP RNA immunoprecipitation experiments done by the O'Neill lab. SINE28 localizes at the centromere throughout the cell cycle by RNA-FISH (Introduction Figure 3-1). Inhibiting SINE28 binding by transfecting human cells with a dsRNA SINE28 oligo caused a reduction in CENP-A levels at centromeres and specifically resulted in a loss of new CENP-A deposition (Introduction Figure 3-2).

We will demonstrate the vertebrate conserved domain of HJURP is required for SINE28 interaction in vivo and in vitro. This conserved region of HJURP, and therefore its interaction with SINE28, is required for HJURP to effectively deposit CENP-A at centromeres. The work presented here provides evidence for a previously uncharacterized role of a non- $\alpha$-satellite RNA in CENP-A deposition. 
Introduction Figure 3-1. SINE28 associates with HJURP and CENP-A and is present at the centromere throughout the cell cycle.

(A) RNA-IP experiment from human cells, pulling down either HJURP or CENP-A then sequencing all associated RNA's. Histograms display the consensus positions of SINE28 that interact most frequently with either HJURP or CENP-A. The Y-axis represents SINE28 frequency, the number of times a particular nucleotide was sequenced. (B) RNAFISH using SINE28 probe on interphase cells (top panel) and mitotic spreads (bottom panel) to display its localization at centromeres and nucleoli throughout the cell cycle. Centromeres are stained with an antibody to CENP-A. DNA is stained with DAPI.

All data in Introduction Figure 3-1 was collected and assembled by Chu Zhang in Rachel O'Neill's lab at the University of Connecticut. 

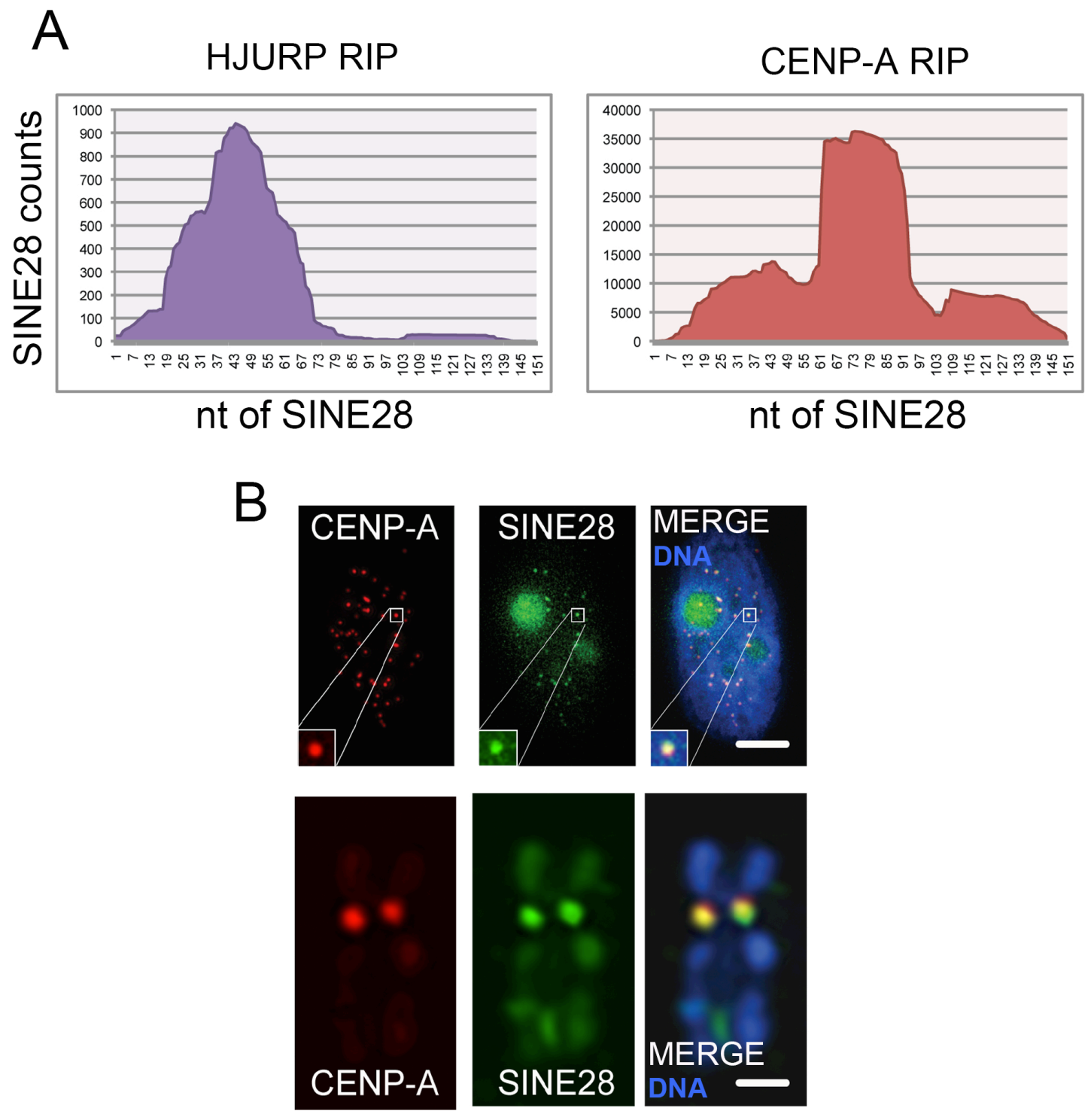
Introduction Figure 3-2. SINE28 is required for CENP-A deposition in human cells.

(A) qRT-PCR in HeLa cells following SINE 28 dsRNA transfection shows increased level of SINE28 progenitor long transcript RNA and decreased level of 40nt SINE28 RNA relative to miR16 control. Relative fold change is shown. (B) Representative CENP-A immunofluorescence (IF) image of control and SINE28 dsRNA transfected telophase/early G1 cells showing impaired CENP-A loading (Blue: DAPI, Green: CENPA). The quantification of CENP-A IF signal (using anti-CENP-A antibody) 24 hours after transfection is shown on the right of the images. Average intensity of CENP-A staining of 100 cells was quantified using Delta Vision WorX software. ${ }^{*} \mathrm{P}<0.01$, Scale bar: 5 $\mu \mathrm{m}$. (C) Milestone of double thymidine block and CENP-A-SNAP-tag assay. (D) CENPA-SNAP-tag image showed no CENP-A loading 24 hours after SINE28 transfection. Blue: DAPI, Green: cdt, Red: CENP-A. Scale bar: $5 \mu \mathrm{m}$.

All data in Introduction Figure 3-2 was collected and assembled by Chu Zhang in Rachel O'Neill's lab at the University of Connecticut. 
A

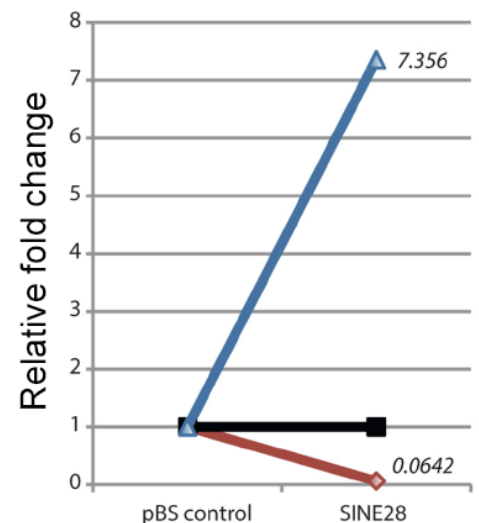

$\approx$ SINE28

RPIIIsRNA

- non-targeted

RPIIIsRNA

SINE28

ong transcript

C

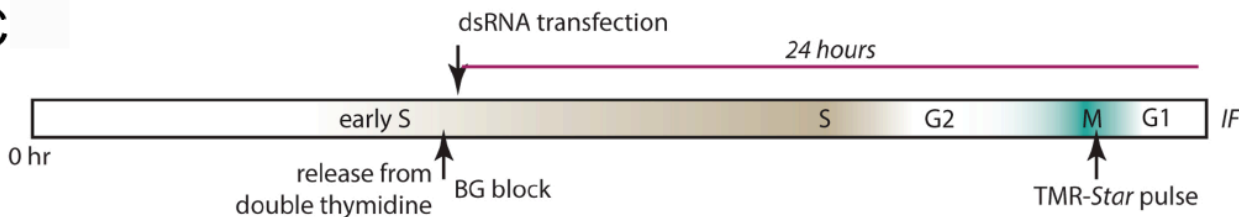

D
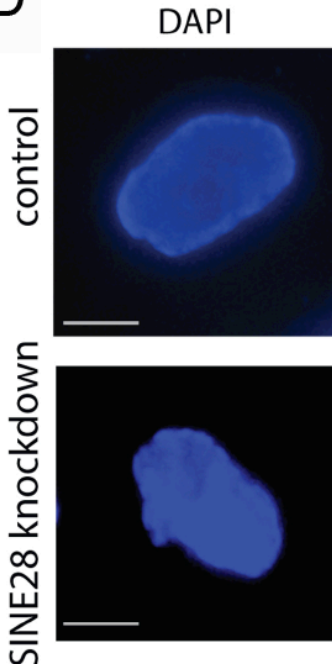

B

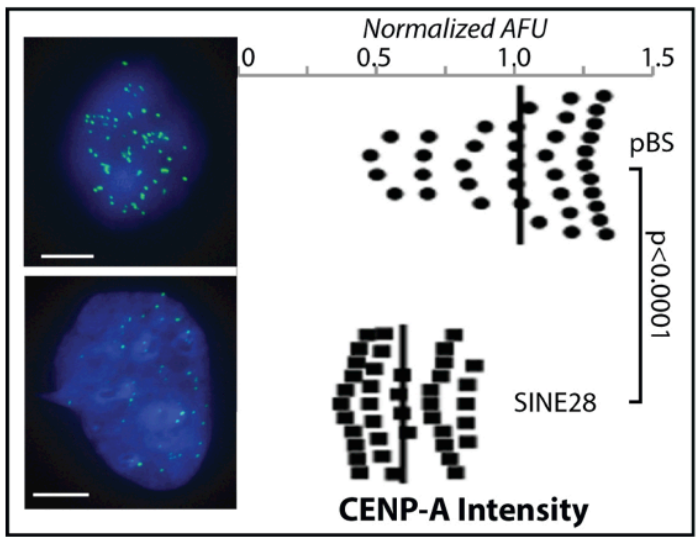

SNAP-tag

$c d t$
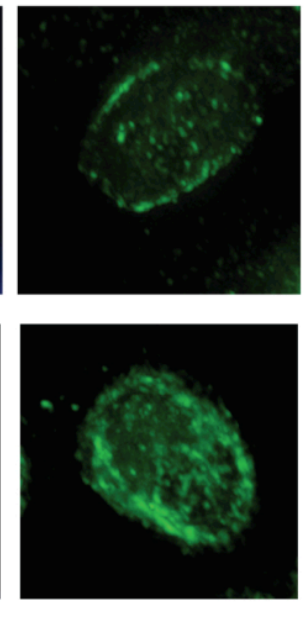

CENP-A
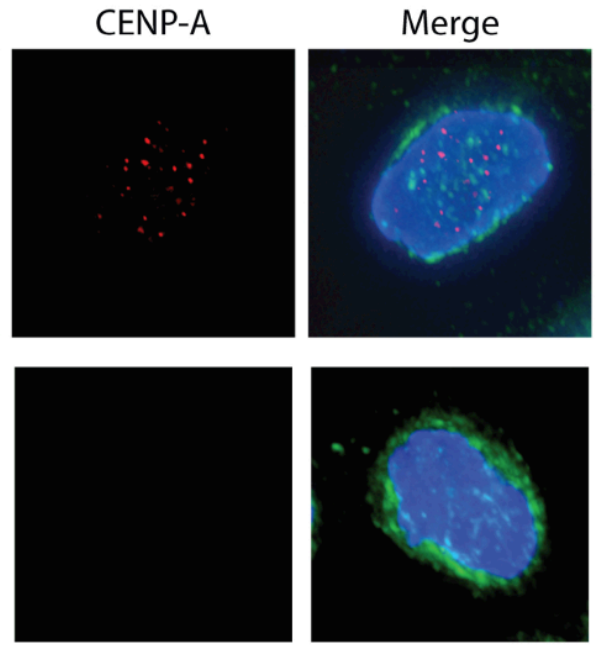


\section{$\underline{\text { Results }}$}

HJURP vertebrate conserved domain is responsible for HJURP's nucleolar localization

Human HJURP has a central domain that is conserved among vertebrate members of the HJURP proteins (Sanchez-Pulido et al., 2009). To discern the function of the conserved region of human HJURP, we generated a deletion mutant of HJURP that lacked amino acids 208-350 ( $\mathrm{HJ}^{\Delta 208-350}$-GFP), which fully removed the annotated conserved domain (Figure 3-1 A). Full length HJURP (HJ'-748-GFP) has two localization patterns in human cells, a nucleolar pool that is present throughout the cell cycle and a centromere-localized pool that only exists in early G1 during CENP-A deposition (Dunleavy et al., 2009; Foltz et al., 2009) (Figure 3-1 B). We observed that unlike full length $\mathrm{HJ}^{1-748}$-GFP, $\mathrm{HJ}^{\Delta 208-350}$-GFP was unable to localize to nucleoli (Figure 3-1 B). Full length $\mathrm{HJ}^{1-748}$-GFP shows 1.6 fold enrichment in the nucleoli, and CENP-A accompanies it there, also showing a significant enrichment in nucleoli. Conversely, $\mathrm{HJ}^{\Delta 208-350}$-GFP, is occluded from the nucleoli so that its intensity is less there than in the general nucleus (Figure 3-1 C). Using the nucleolar localization signal search tool, NoD, a nucleolar localization signal is predicted within this conserved region of HJURP (Suppl. Figure 3-1 A, B) (Scott et al., 2010; Scott et al., 2011). Loss of this nucleolar localization signal likely contributes to the loss of nucleolar localization, however the remaining portion of the deleted region in $\mathrm{HJ}^{\Delta 208-350}$-GFP may also encourage nucleolar localization, perhaps through RNA binding.

We next wanted to investigate if the HJURP conserved domain, $\mathrm{HJ}^{201-348}$, is sufficient to localize to nucleoli on its own. $\mathrm{HJ}^{201-348}$ displayed four localization patterns 
in live cells, but only two of these localization patterns were stable following preextraction and fixation (Suppl. Figure 3-3 A, C). Upon pre-extraction and fixation, 100\% of $\mathrm{HJ}^{201-348}$-GFP positive cells showed nucleolar staining, but these could be classified into two sub-groups. $65 \%$ of U2OS cells showed only nucleolar localization of $\mathrm{HJ}^{201-348}$ GFP (Suppl. Figure 3-3 C, D). The remaining 35\% of the cells showed a speckled, noncentromeric localization pattern in addition to nucleolar localization. (Suppl. Figure 3-3 C, D). The identity of these speckles is not known, but they resemble paraspeckles ribonucleoprotein bodies found in mammalian cell nuclei that harbor proteins with RNA processing or binding capabilities. Investigation of whether these speckles overlap with DBHS proteins would confirm their identity as paraspeckles (Bond and Fox, 2009). We conclude that $\mathrm{HJ}^{201-348}$ is sufficient for nucleolar localization, thus confirming the predicted NoLS (Suppl. Figure 3-1) within the conserved domain of HJURP.

We next wanted to determine if the centromere localization of HJURP was affected by the nucleolar localization loss seen with $\mathrm{HJ}^{\Delta 208-350}$-GFP. We know from previous studies that the HCTD1 (HJURP C-Terminal Domain 1) of HJURP enables its centromere localization (Zasadzinska at al. 2013). $\mathrm{HJ}^{\Delta 208-350}$-GFP still contains this HCTD1 (R1) domain, so should still be able to localize to centromeres. To investigate this, Hela-TRex stable cells lines expressing either $\mathrm{HJ}^{1-748}$-GFP or $\mathrm{HJ}^{\Delta 208-350}$-GFP were created. In randomly cycling cells, $\mathrm{HJ}^{\Delta 208-350}$-GFP localized to centromeres in a comparable percentage of cells to $\mathrm{HJ}^{1-748}$-GFP (Figure 3-1 D, E), suggesting that nucleolar localization of HJURP is not required for its centromere targeting.

In addition to being able to localize to centromeres, HJURP must also be able to bind to CENP-A for successful CENP-A deposition to occur. The Scm3 domain of 
HJURP enables it to bind to CENP-A and is sufficient to recruit CENP-A to noncentromeric sites (Barnhart et al., 2011; Shuaib et al., 2010). To verify that $\mathrm{HJ}^{\Delta 208-350}$ GFP, which contains an intact Scm3 domain (Figure 3-1 A), can still bind to CENP-A, HEK293T cells were transfected for 24 hours with mCherry-CENP-A and either $\mathrm{HJ}^{1-748}$ GFP or $\mathrm{HJ}^{\Delta 208-350}$-GFP. The GFP constructs were immunoprecipitated using a GFP antibody and probed for association with mCherry-CENP-A. $\mathrm{HJ}^{\Delta 208-350}-\mathrm{GFP}$ was able to pull down a comparable amount of mCherry-CENP-A as $\mathrm{HJ}^{1-748}$-GFP verifying that the conserved domain is not involved in the HJURP-CENP-A interaction (Suppl. Figure 3-2). In conclusion, though HJURP lacking its conserved domain $\left(\mathrm{HJ}^{\Delta 208-350}\right)$ no longer localizes to nucleoli, it can still fulfill its crucial functions of localizing to centromeres and binding to CENP-A (Figure 3-1; Suppl. Figure 3-2).

Npm1 is a known member or the CENP-A pre-nucleosomal complex (Dunleavy et al., 2009; Foltz et al., 2009; Shuaib et al., 2010). Npm1 also enriches in nucleoli similarly to HJURP. As such, observing the nucleolar localization loss upon deleting the vertebrate conserved domain of HJURP led us to predict this region of the protein was also required for Npm1 binding. Combinations of targeting HJURP fragments to the $\mathrm{LacO}$ array and immunoprecipitation experiments suggested that the conserved domain was important for Npm1 interaction (Suppl. Figure 3-4). However, I could never observe a black and white loss of Npm1 binding in immunoprecipitation experiments using $\mathrm{HJ}^{\Delta 208-350}$. That being said, targeting the conserved domain of HJURP $\left(\mathrm{HJ}^{201-348}\right)$ alone to the LacO array was sufficient to recruit Npm1 at levels comparable to the full length HJURP protein (Suppl. Figure 3-4 A-C). We decided to focus our investigations on 
characterizing the conserved domain of HJURP as an RNA binding domain, but we continue to consider the role of Npm1 interaction with HJURP. 
Figure 3-1. HJURP's vertebrate conserved domain is required for its nucleolar localization.

(A) Schematic of full length $\mathrm{HJ}^{1-748}$ and $\mathrm{HJ}^{\Delta 208-350}$ (vertebrate conserved domain deletion mutant) domain structure. (B) Representative live cell images of HeLa cells transfected for 24 hrs with mCherry-CENP-A and either $\mathrm{HJ}^{1-748}$-GFP or $\mathrm{HJ}^{\Delta 208-350}$-GFP. A representative image is provided for both centromere-localized HJ cells (early G1) and non-centromere localized cells to distinguish nucleolar localization loss from centromeric localization. Scale bar represents $5 \mu \mathrm{m}$. (C) Quantification of HJ nucleolar localization. Graph represents a ratio of the average nucleolar intensity for GFP (gray) and CENP-A (white) to the average nuclear intensity in $\mathrm{HJ}^{1-748}$ or $\mathrm{HJ}^{\Delta 208-350}$ transfected HeLa cells. $\mathrm{n}>15$ measurements per condition, $\mathrm{N}=2$ experiments. Error bars represent standard deviation. Mann-Whitney test between the average GFP or CENP-A ratios yields $\mathrm{p}<$ 0.0001 and $\mathrm{p}=0.0002$, respectively. (D) Representative fixed cell images of HeLa TRex cell lines stably expressing either $\mathrm{HJ}^{1-748}$-GFP or $\mathrm{HJ}^{\Delta 208-350}$-GFP demonstrating that $\mathrm{HJ}^{\Delta 208-350}$ is still able to localize to centromeres in a comparable number of cells as $\mathrm{HJ}^{1-}$ ${ }^{748}$. Cells are stained with an antibody to endogenous CENP-A. Scale bar represents 5 $\mu \mathrm{m}$. (E) Quantification of centromere localization of GFP-tagged HJ stable lines represented in D. Percentages are from randomly cycling cell populations. $\mathrm{n}=100$ cells counted, $\mathrm{N}=3$ experiments. Error bars represent standard deviation. $\mathrm{T}$-test yields $\mathrm{p}=0.41$ showing there is no difference from $\mathrm{HJ}^{1-748}$ in the percentage of cells with $\mathrm{HJ}^{\Delta 208-350}$ at centromeres. 

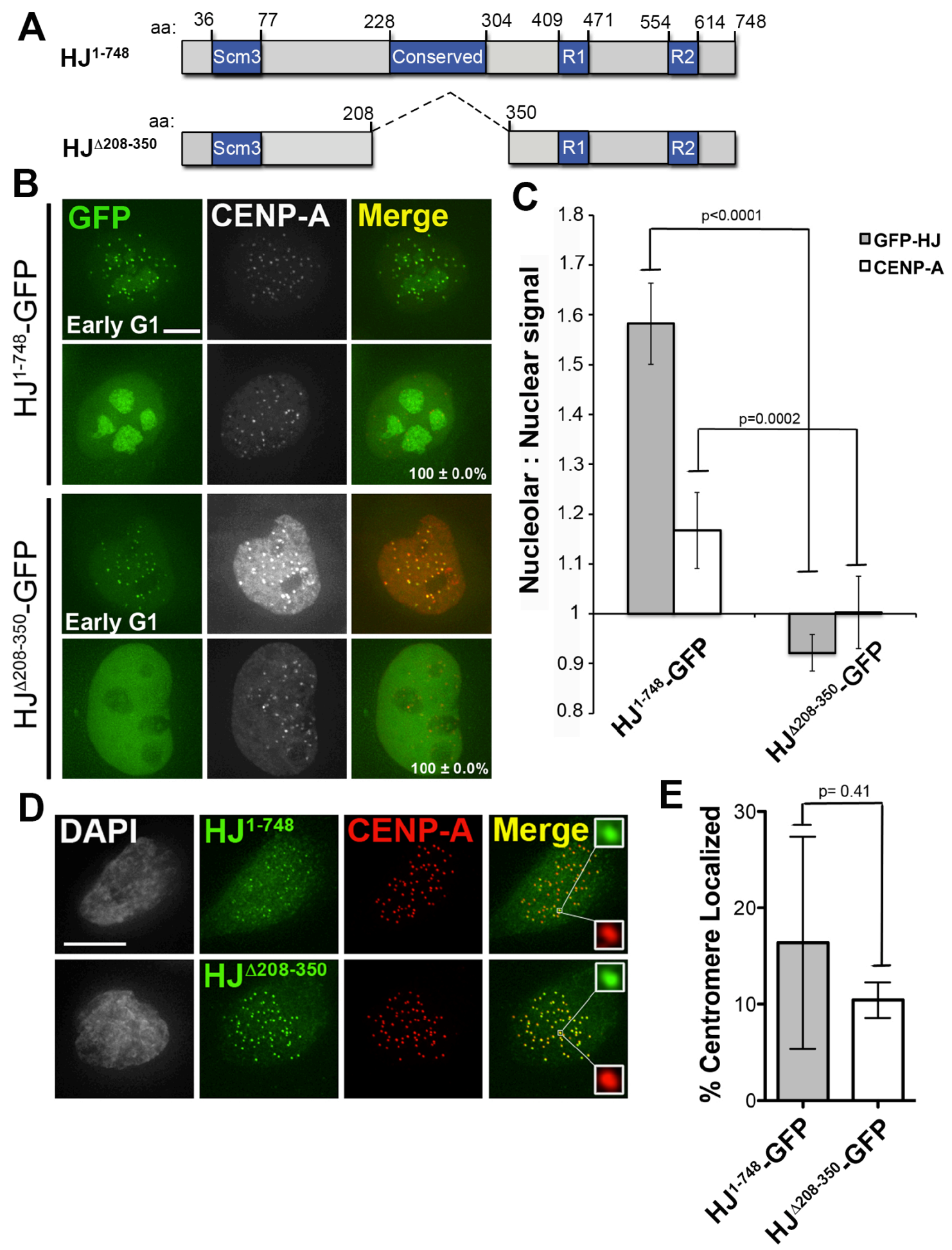
HJURP vertebrate conserved domain is required for SINE28 binding in vivo

As the major site of rRNA transcription and processing, nucleoli are highly concentrated in both rRNA and other nuclear RNA's (Mao et al., 2011). The requirement for the conserved domain of HJURP in its nucleolar localization led us to investigate if the conserved domain of HJURP contains an RNA binding motif. We specifically wanted to know if the conserved domain was important for HJURP's interaction with the centromere-enriched SINE28 ssRNA (Introduction Figure 3-1). SINE28 is required for new CENP-A deposition at centromeres, but the mechanism why is not understood (Introduction Figure 3-2).

Because the SINE28 ssRNA enriches at centromeres (Introduction Figure 3-1), we took advantage of a protein sequestration technique to analyze the isolated interaction between HJURP and SINE28. Using a U2OS cell line with a stably integrated, noncentromeric LacO/TRE element, a protein of interest can be tethered away from its usual location by fusing it with LacI, and its interaction partners can then be analyzed in isolation from its endogenous environment (Janicki et al., 2004). U2OS-LacO cells were transfected with mCherry-LacI-HJ ${ }^{1-748}$, mCherry-LacI-HJ ${ }^{1-208}$, mCherry-LacI-HJ ${ }^{1-348}$, mCherry-LacI-HJ ${ }^{\Delta 208-350}$, or empty vector as a negative control. Two of these HJURP fragments contained the conserved domain of HJURP, $\mathrm{HJ}^{1-748}$ and $\mathrm{HJ}^{1-348}$, while the other two did not. RNA-FISH was then performed on these cells using a probe that specifically binds the sequence of SINE28 (Figure 3-2). HJURP fragments containing the vertebrate conserved domain $\left(\mathrm{HJ}^{1-748}\right.$ and $\left.\mathrm{HJ}^{1-348}\right)$ were both able to recruit SINE28 to the LacO array as indicated by positive SINE28 FISH signal at the array. Interestingly, HJURP fragments lacking the conserved domain $\left(\mathrm{HJ}^{1-208}\right.$ and $\left.\mathrm{HJ}^{\Delta 208-350}\right)$ were unable to interact 
with SINE28 in vivo (Figure 3-2 A). Consistent with the conserved domain of HJURP being required for SINE28 interaction, the amount of SINE28 FISH signal was significantly enriched at LacO arrays targeted with both full-length HJURP $\left(\mathrm{HJ}^{1-748}\right)$ and HJURP containing the $\mathrm{Scm} 3$ and conserved domains of the protein $\left(\mathrm{HJ}^{1-348}\right)$ (Figure 3-2 B). CENP-A also interacts with SINE28, though it binds a distinct region of the RNA than HJURP (Introduction Figure 3-1). Targeting LacI-HJ ${ }^{1-208}$ to the LacO array is known to recruit CENP-A to the array (Barnhart et al., 2011). The small amount of FISH signal seen at the LacI-HJ ${ }^{1-208}$ arrays, indicated by their array enrichment values of slightly greater than 1, can be explained by the recruited CENP-A binding to SINE28. However, these HJURP constructs lacking the conserved domain displayed SINE28 FISH signals that were not significantly different from targeting the mCherry-LacI vector alone to the array (Figure 3-2 B). Therefore, we are confident the primary binding event we are assaying at the array is between HJURP and SINE28 due to the large concentration of the over-expressed HJURP proteins tethered there. We conclude the conserved domain of HJURP is required for its interaction with SINE28 ssRNA in vivo. 
Figure 3-2. SINE28 requires the vertebrate conserved domain to interact with HJURP in vivo.

(A) U2OS-LacO cells were transfected for 48 hours with mCherry-LacI empty vector, mCherry-LacI-HJ ${ }^{1-748}$, mCherry-LacI-HJ ${ }^{1-208}$, mCherry-LacI-HJ ${ }^{\Delta 208-350}$, or mCherry-LacI$\mathrm{HJ}^{1-348}$. Cells were fixed then RNA-FISH was performed with a probe for SINE28. DNA is stained with DAPI. (B) Quantification of RNA-FISH experiment in A. SINE28 intensity at array was measured then set as a ratio over the average nuclear background signal. Arrays with no enrichment show a value of 1 . LacI-HJ ${ }^{1-348}$ and LacI-HJ ${ }^{1-748}$ both showed SINE28 enrichment that was statistically significant $(\mathrm{p}<0.05$ and $\mathrm{p}<0.01$ respectively) as compared to LacI empty vector using a Kruskal-Wallis test followed by a Dunn's multiple comparison test. $\mathrm{n} \geq 25$ arrays measured per condition, $\mathrm{N}=3$ biological replicates.

RNA-FISH and imaging was done by Chu Zhang at the University of Connecticut. Cells were transfected and image analysis was done by MCD. 


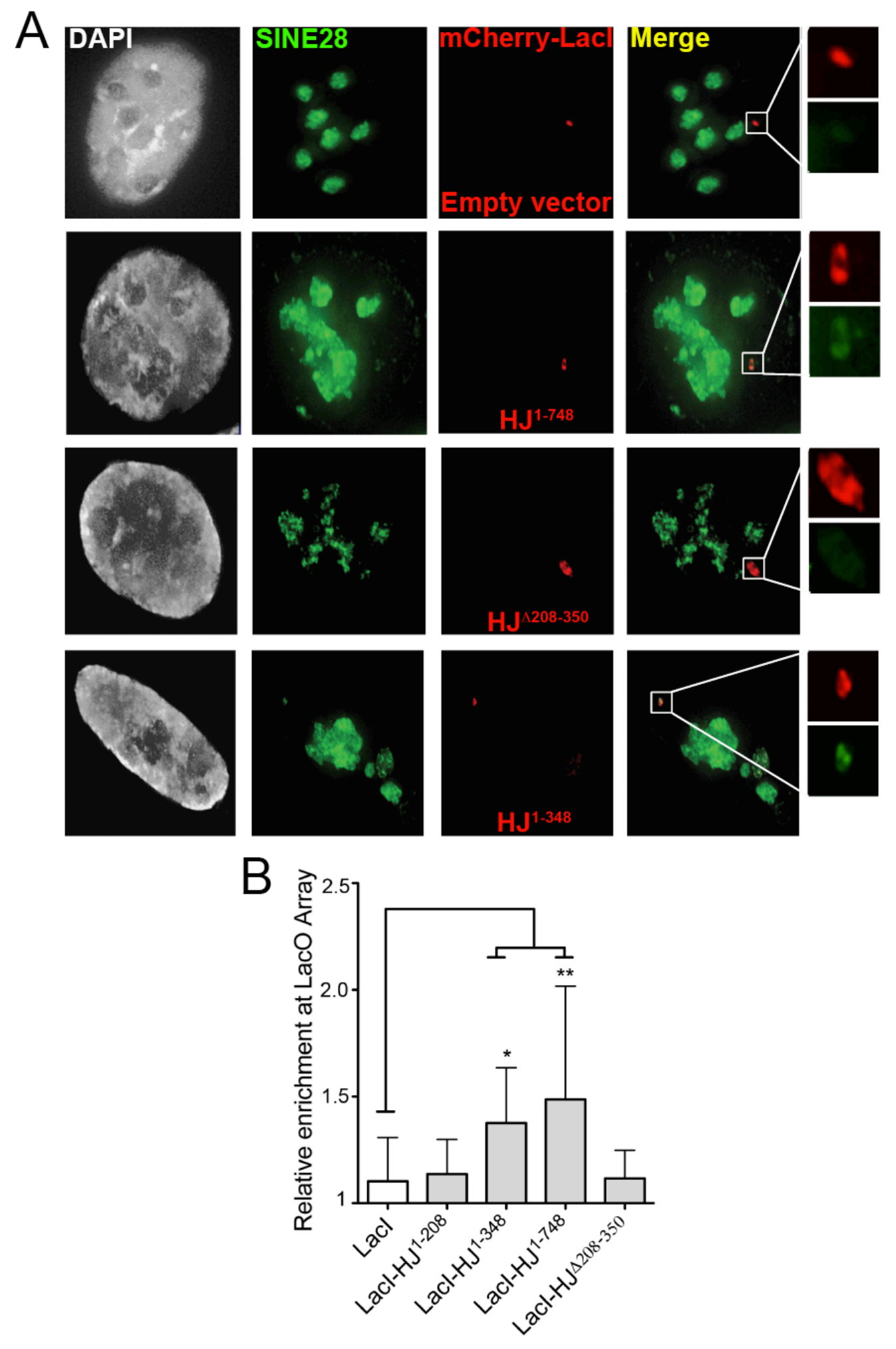


HJURP vertebrate conserved domain is required for SINE28 binding in vitro

The SINE28 FISH analysis revealed the conserved domain of HJURP is required for binding between HJURP and SINE28 in vivo (Figure 3-2). To verify that the conserved domain is the direct interaction site between HJURP and SINE28, we did an in vitro pull down assay with MBP-HJ ${ }^{1-748}, \mathrm{MBP}-\mathrm{HJ}^{\Delta 208-350}, \mathrm{MBP}^{-\mathrm{HJ}^{1-348}}$, or MBP alone using a biotinylated version of the SINE28 ssRNA. Because this is an in vitro assay, the components of the reaction are controlled, and thus one can determine direct interactions. Consistent with the RNA FISH results, $\mathrm{HJ}^{1-748}$ and $\mathrm{HJ}^{1-348}$ were both sufficient to bind to the SINE28 RNA in vitro. In contrast, $\mathrm{HJ}^{\Delta 208-350}$ was unable to bind to SINE28 RNA, similarly to what was seen in the RNA FISH experiment (Figure 3-3 A). Adding CENP$\mathrm{A} / \mathrm{H} 4$ heterotetramer into the reaction with $\mathrm{MBP}-\mathrm{HJ}^{1-348}$ enhanced the amount of $\mathrm{HJ}^{1-348}$ that could pull down with the RNA. This is not well understood yet, but because CENPA also binds to the RNA, this may suggest a synergistic binding between CENP-A, HJURP, and the RNA (Figure 3-3 A). No gross differences were observed in the binding efficiencies of HJURP for the sense or antisense strands of the SINE28 RNA, arguing that the HJURP conserved domain may recognize the secondary structure of the RNA, instead of a specific sequence. These data in combination with the RNA FISH results provide strong evidence that endogenous SINE28 binds to HJURP in its vertebrate conserved domain in vivo and that this interaction is direct as indicated by loss of $\mathrm{HJ}^{\Delta 208-}$ ${ }^{350}$ binding in vitro. Additional experiments are in progress to establish the specificity of HJURP for SINE28 RNA over a non-specific RNA in vitro. 
Figure 3-3. HJURP binds SINE28 RNA in vitro via its vertebrate conserved domain.

(A) In vitro RNA binding experiment using recombinant MBP-HJURP ${ }^{1-748}$, MBPHJURP $^{\Delta 208-350}$, MBP-HJURP ${ }^{1-348}$, or MBP alone. For the MBP-HJ ${ }^{1-348}$ condition, this was done with or without recombinant CENP-A/H4 heterotetramer. Sense (S) or anti-sense (AS) SINE28 biotinylated RNA was incubated with the MBP proteins, with or without CENP-A/H4 heterotetramer in the case of MBP-HJ ${ }^{1-348}$. The RNA was then pulled down using streptavidin-coated beads, bringing along any bound proteins. Pull-down fractions were blotted for MBP and CENP-A. N=2 replicates

In vitro pull-down experiment was done by Isaac Nardi. 


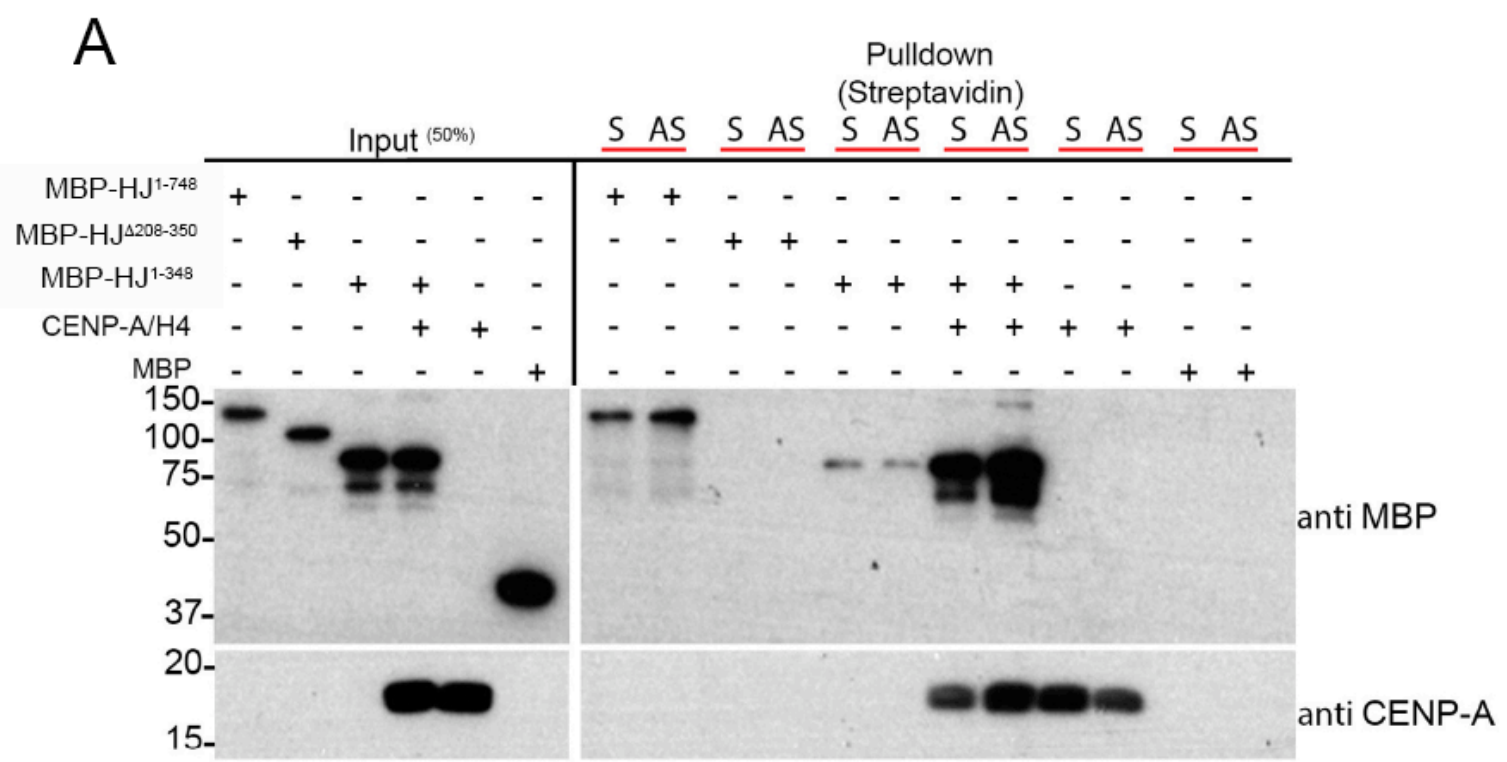


HJURP conserved domain is required for stable CENP-A deposition at centromeres

Transfecting HeLa cells with SINE 28 dsRNA to inhibit SINE28 resulted in a reduction in overall CENP-A levels at the centromere (Introduction Figure 3-2). This supports a positive role for SINE28 in CENP-A deposition or stability at the centromere, but it was unclear if this phenotype was due to loss of SINE28 interaction with HJURP, CENP-A, or both. Because HJ ${ }^{\Delta 208-350}$ cannot bind to SINE28 RNA (Figure 3-2; Figure 33), but it still retains centromere targeting and CENP-A binding (Figure 3-1 D, E; Suppl. Figure 3-2), it allowed us to specifically investigate if SINE28 interaction with HJURP is important for CENP-A deposition.

We used cell lines stably expressing $\mathrm{HJ}^{1-748}$-GFP, $\mathrm{HJ}^{\Delta 208-350}$-GFP or an un-rescued parental cell line to perform knockdown and rescue experiments when endogenous HJURP was depleted from the cell and assessed the steady state levels of centromeric CENP-A. Cells were treated for 48 hours with siRNA to the 5' and 3' UTR sequences of HJURP to effectively deplete the endogenous HJURP while not affecting the expression of the rescue GFP constructs. Endogenous HJURP was depleted to $10-25 \%$ of steady state levels, and rescue construct expression was unaffected by HJURP depletion (Figure 3-4 A). We then investigated the steady state endogenous CENP-A levels by staining with a CENP-A antibody and measuring its intensity under these conditions. Steady state centromeric CENP-A levels were approximately equal between the parental, $\mathrm{HJ}^{\Delta 208-350}$ GFP, and the $\mathrm{HJ}^{1-748}$-GFP cell lines when the cells were treated with negative control siRNA (Figure 3-4 B, D). Upon endogenous HJURP depletion, we observed that the exogenous $\mathrm{HJ}^{1-748}$-GFP was able to fully rescue CENP-A levels (Figure 3-4 C, D). Conversely, $\mathrm{HJ}^{\Delta 208-350}$-GFP was deficient at rescuing steady state centromeric CENP-A 
levels (Figure 3-4 C, D). The CENP-A intensity in the $\mathrm{HJ}^{\Delta 208-350}$-GFP cells when HJURP was depleted was equivalent to the CENP-A levels in the parental cells, which contained no exogenous rescue construct (Figure 3-4 C, D).

This experiment indicates that despite successfully targeting to centromeres and binding to CENP-A, HJURP ${ }^{\Delta 208-350}$ is unable to rescue endogenous HJURP depletion. This mimics the phenotype observed for blocking SINE28 function (Introduction Figure 3-2) and argues that SINE28's role in CENP-A deposition is mediated by its interaction with the HJURP vertebrate conserved domain. 
Figure 3-4. HJURP lacking amino acids 208-350 is unable to rescue CENP-A deposition at centromeres when endogenous HJURP is depleted.

(A) Western blots of cell lysates from HeLa-TRex parental cells or HeLa-TRex cell lines stably expressing $\mathrm{HJ}^{1-748}$-GFP or $\mathrm{HJ}^{\Delta 208-350}$-GFP. Cells were treated for $48 \mathrm{hrs}$ with 40 nM HJ 5' and $3^{\prime}$ UTR siRNA or $40 \mathrm{nM}$ negative control siRNA prior to harvesting. Blots are probed with antibodies to HJURP (top blot) and tubulin (bottom blot) to show endogenous HJURP depletion. (B) and (C) Immunofluorescence of same cell lines as in A (parental HeLa TRex, HeLa TRex HJ ${ }^{1-748}$-GFP, or HeLa TRex $\mathrm{HJ}^{\Delta 208-350}$-GFP). Cells were treated in parallel to cells in A for $48 \mathrm{hrs}$ with $40 \mathrm{nM}$ negative control siRNA (B) or $40 \mathrm{nM} \mathrm{HJ} 5$ ' and 3' UTR siRNA (C) prior to fixing and staining. Cells were stained with a monoclonal antibody to CENP-A (red), GFP constructs are shown in green, and nuclei are stained with DAPI. Scale bars represent $5 \mu \mathrm{m}$. (D) Centromeric intensity analysis of coverslips represented in B and C. Bottom and top lines of boxes represent the 25th and 75th percentiles, respectively, and the middle line is the median. The whiskers display the 2.5 to 97.5 percentiles. Outliers are represented as points. $n=80$ centromeres per condition, $\mathrm{N}=2$ biological replicates. Kruskal-Wallis test yields $\mathrm{p}<0.0001$ between the medians of conditions marked with blue asterisks and the negative control siRNA condition. 


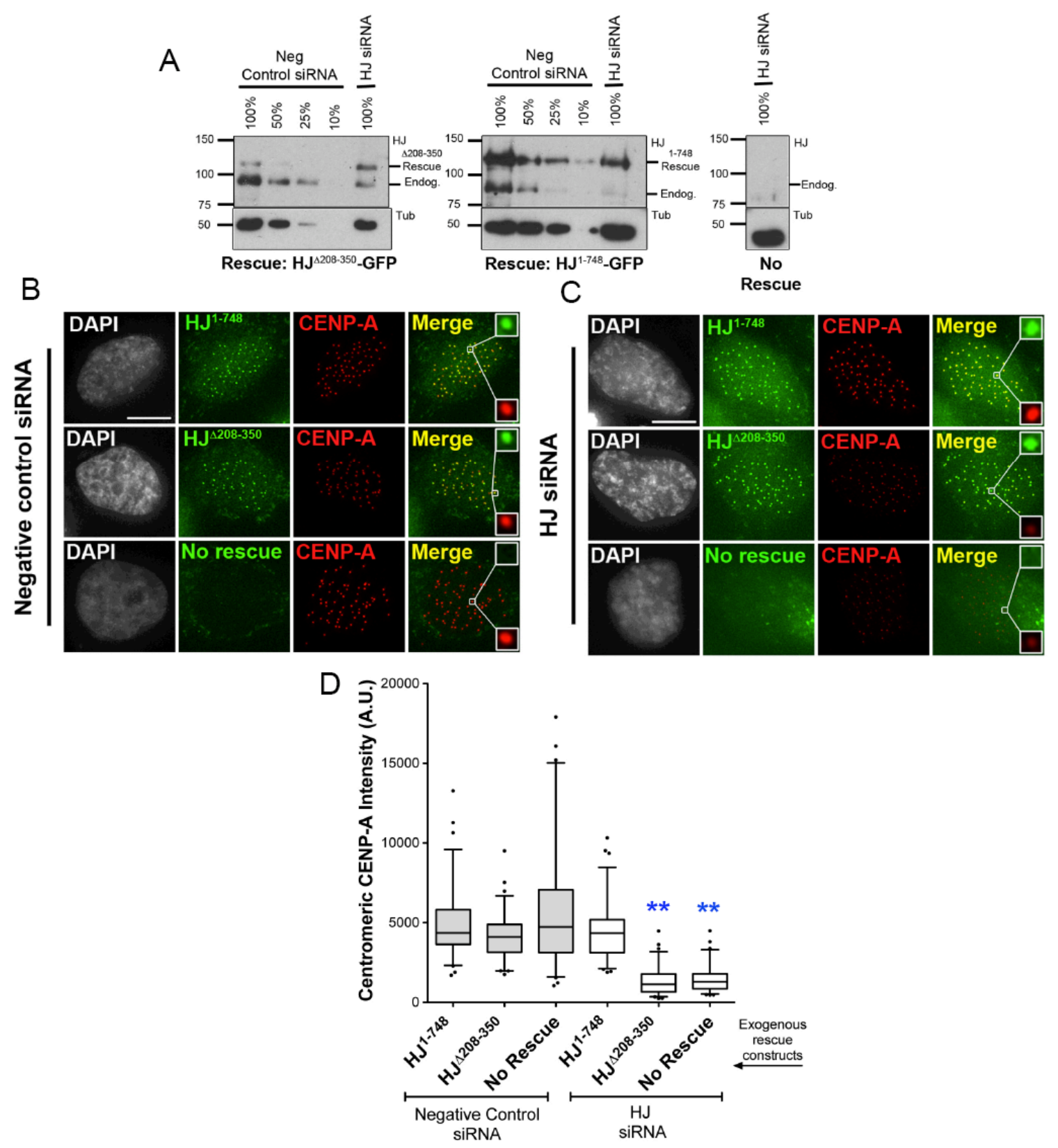


$H^{4208-350}$ cannot rescue new CENP-A loading

Blocking SINE28 function by transfecting in a complementary double-stranded oligo prevented new CENP-A loading using a SNAP-labeled CENP-A cell line (Introduction Figure 3-2). In Figure 3-4, endogenous HJURP depletion and rescue with HJURP $^{\Delta 208-350}$ resulted in a reduction in overall CENP-A levels at centromeres. Measuring steady state levels of CENP-A as was done in Figure 3-4 cannot distinguish between a change in CENP-A stability at centromeres and a defect in new CENP-A deposition. To look specifically at the fate of newly synthesized CENP-A during the knockdown and rescue experiment, $\mathrm{HJ}^{1-748}$-GFP, $\mathrm{HJ}^{\Delta 208-350}$-GFP or parental HeLa-TRex lines were co-transfected with HJURP 5' and 3' UTR siRNA and an mCherry-CENP-A expressing plasmid. Cells were treated with a second round of siRNA at 48 hours to ensure endogenous HJURP depletion and were analyzed at 72 hours to look for newly synthesized mCherry-CENP-A loading at centromeres. Endogenous HJURP was depleted to $10-25 \%$ of steady state levels (Figure 3-5 A). mCherry-CENP-A was efficiently loaded at centromeres in all negative control treated conditions (Figure 3-5 B, C). However, when endogenous HJURP was depleted, only cells exogenously expressing $\mathrm{HJ}^{1-748}$-GFP could rescue newly synthesized mCherry-CENP-A deposition at centromeres. $\mathrm{HJ}^{\Delta 208-350}$ GFP and un-rescued parental cells showed no newly synthesized mCherry-CENP-A assembly at centromeres (Figure 3-5 B, C).

There are a few possibilities for why $\mathrm{HJ}^{\Delta 208-350}$ is unable to rescue new CENP-A deposition. One possibility is the RNA binds to CENP-A to work alongside HJURP in shielding DNA interaction sites on CENP-A, thus preventing aberrant binding. Another possibility is SINE28 may stabilize HJURP and CENP-A/H4. This is consistent with 
preliminary SNAP-CENP-A labeling studies suggesting that newly synthesized CENP-A localizes to the nucleolus prior to being deposited at the centromere (Suppl. Figure 3-5). Co-expressing mCherry-CENP-A with $\mathrm{HJ}^{\Delta 208-350}$-GFP also caused CENP-A exclusion from nucleoli, suggesting that CENP-A's ability to transit the nucleolus may require HJURP enrichment there (Figure 3-1 B, C). If nucleolar transit prior to deposition were a required step in CENP-A deposition, it would be disrupted in cells expressing $\mathrm{HJ}^{\Delta 208-350}$ GFP and could contribute to the loss of new CENP-A deposition observed. SINE28 is also enriched at nucleoli (Introduction Figure 3-1 B), so the interaction between HJURP, CENP-A, and SINE28 may require nucleolar localization.

Alternatively, SINE28 association with HJURP through its conserved domain may be required for HJURP's nucleosome assembly activity during new CENP-A deposition. This could occur through SINE28 stabilizing HJURP's and/or other CENP-A deposition machinery's localization at the centromere during CENP-A loading. RNA is known to stabilize the centromeric localization of CENP-C and CENP-W as well as members of the CPC (Chun et al., 2011; Ferri et al., 2009; Wong et al., 2007), so this is an established role of RNA at the centromere. Further studies are in progress to understand why SINE28 and the vertebrate conserved domain of HJURP are required for new CENP-A deposition at human centromeres. 
Figure 3-5. $H J^{4208-350}$-GFP is not sufficient to rescue new CENP-A loading.

(A) Western blots of cell lysates from HeLa-TRex parental cells or HeLa-TRex cell lines stably expressing $\mathrm{HJ}^{1-748}$-GFP or $\mathrm{HJ}^{\Delta 208-350}$-GFP. Cells were treated for 48 hrs with 20 nM HJURP 5' and 3' UTR siRNA or $40 \mathrm{nM}$ Negative control siRNA and were transfected with an mCherry-CENP-A expressing plasmid prior to harvesting. (B) Cells treated in parallel to those blotted in A. As in A, the cells were co-transfected with a plasmid expressing mCherry-CENP-A along with the $48 \mathrm{hr} 20 \mathrm{nM}$ HJURP 5' and 3' UTR siRNA or Negative control siRNA treatment. Cells were then fixed and analyzed for centromeric localization of the exogenous mCherry-CENP-A, which was only synthesized when endogenous HJ was depleted, so any centromere localized mCherryCENP-A must have been loaded by the rescue GFP constructs. Cells were stained with an antibody to CENP-T as a centromere marker. Scale bars represent $5 \mu \mathrm{m}$. (C) Quantification of centromeric mCherry-CENP-A intensity of cells in B. Asterisks indicate statistically significant differences in the medians between the indicated populations and the negative control condition. $\mathrm{p}<0.0001$ by Kruskal-Wallis test. $\mathrm{n}>50$ centromeres per condition, $\mathrm{N}=2$ biological replicates. 


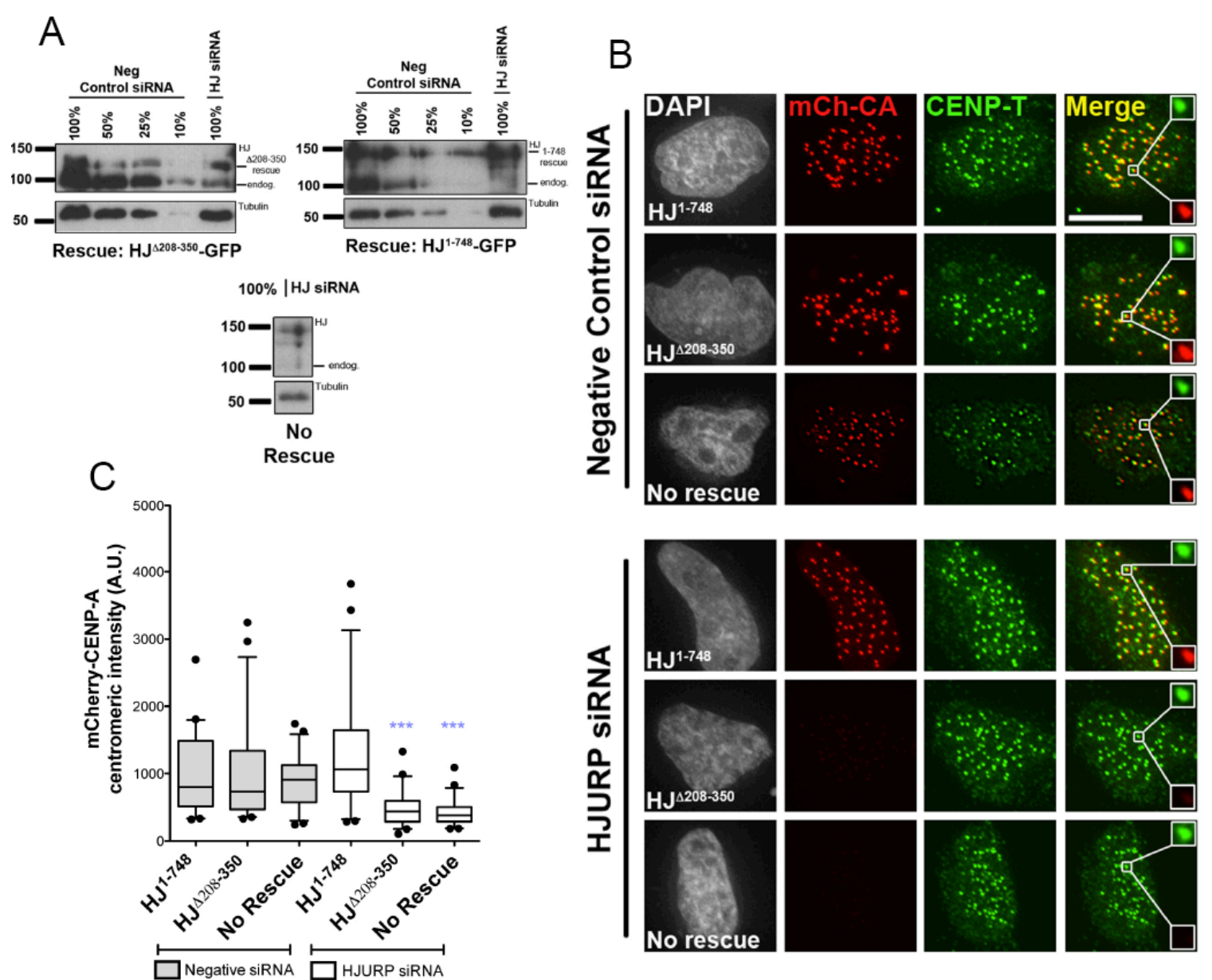


RNaseA+RNaseT1 cocktail treatment reduces HJURP and Mis18a centromeric recruitment

Based on RNA-IP experiments, SINE28 is the most abundant RNA bound to CENP-A and HJURP (O'Neill Lab). SINE28 is also highly enriched at the centromere throughout the cell cycle (Introduction Figure 3-1). Similarly, alpha-satellite transcripts are also known to enrich at centromeres in human cells (Wong et al., 2007). We therefore hypothesized that RNA may play a role in stabilizing the CENP-A deposition machinery at centromeres during CENP-A loading in early G1. As a preliminary look at the role of RNA in CENP-A deposition machinery stability, permeabilized cells were treated with a cocktail of RNaseA (hydrolyzes RNA at C and U residues) and RNaseT1 (hydrolyzes RNA at G residues) for 20 minutes prior to fixation and staining. RNase treatment was performed on cells stably expressing either $\mathrm{HJ}^{1-748}$-GFP (Figure 3-6 A-F) or Mis18 $\alpha$-GFP (Figure 3-6 G-J).

For the $\mathrm{HJ}^{1-748}$-GFP stable line, G1 pairs with centromeric $\mathrm{HJ}^{1-748}$-GFP signal were imaged and the intensities of their centromeric GFP, CENP-A, and CENP-T signals were measured (Figure 3-6 A-D). Consistent with a role for RNA in new CENP-A deposition, RNase cocktail treatment resulted in a decrease in centromeric $\mathrm{HJ}^{1-748}$-GFP signal (Figure 3-6 B). A decrease in CENP-A intensity was also observed (Figure 3-6 C), but only in the early G1 cells identified by centromeric $\mathrm{HJ}^{1-748}$-GFP localization. Interphase cells (lacking centromeric $\mathrm{HJ}^{1-748}$-GFP) treated with RNase cocktail did not show a decrease in centromeric CENP-A intensity (Figure 3-6 E). This suggests RNA only affects CENP-A stability at the centromere prior to its deposition into chromatin. CENP-T levels also did not decrease when cells were treated with RNase cocktail (Figure 
3-6 D, F). This is consistent with HJURP, which is bound to pre-nucleosomal CENP-A, requiring RNA for its stability at the centromere during CENP-A deposition. Similar results were obtained when cells were treated with RNaseA alone (Suppl. Figure 3-6).

Because Mis18 lies upstream of HJURP recruitment, we investigated the effect of RNase treatment on Mis18 $\alpha$ centromeric stability. G1 pairs with centromeric Mis18 $\alpha$ GFP signal were imaged and the intensities of their centromeric GFP, CENP-A, and CENP-T signals were measured (Figure 3-6 G-J). Mis18 $\alpha$-GFP was drastically reduced from centromeres following RNase cocktail treatment (Figure 3-6 G, H). CENP-A and CENP-T levels were unchanged in the Mis18a-GFP stable lines following RNase cocktail treatment (Figure 3-6 I, J). CENP-A levels were decreased in the $\mathrm{HJ}^{1-748}$-GFP cells only in the cells where $\mathrm{HJ}^{1-748}$-GFP was at centromeres (Figure 3-6 C vs. E). This indicates the CENP-A that was destabilized by RNase treatment was not deposited yet and was still bound to HJURP. CENP-A levels not changing in Mis18a cells following RNase treatment may be due to HJURP and its pre-nucleosomal CENP-A cargo not yet being present at the centromere in the cells measured.

We conclude that RNA influences Mis18 stability at centromeres. This could in turn affect HJURP stability at centromeres. Alternatively, RNA at the centromere may influence both Mis 18 and HJURP stability in separate ways. The specific role for SINE28 RNA in HJURP and Mis18 $\alpha$ stability needs to be investigated using a SINE28 function blocking oligo instead of using this less-elegant RNase treatment. This general RNase treatment will affect all RNA's present at the centromere, including the alpha-satellite transcripts known to be present there. Therefore, specifically assaying the contribution of SINE28 to Mis $18 \alpha$ and HJURP stability at the centromere is an important next step to 
solidify the current observations. Despite this caveat, the reduction in both of these CENP-A deposition pathway proteins following RNase treatment, and the loss of CENP-

A loading at centromeres observed with an RNA binding mutant of HJURP $\left(\mathrm{HJ}^{\Delta 208-350}\right)$ are together indicative of a critical role for RNA in CENP-A deposition. 


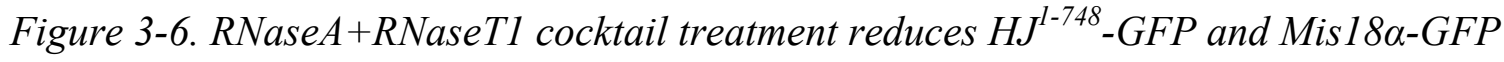
centromeric recruitment.

(A) Representative images of HeLa TRex cells stably expressing GFP-tagged $\mathrm{HJ}^{1-748}$. Cells were treated with a cocktail of RNaseA+RNaseT1 for 20 minutes prior to fixation and staining. Cells were stained with antibodies to CENP-A and CENP-T as centromere markers, and DNA is stained with DAPI. (B) Quantification of centromeric GFP signal of cells represented in A. G1 pairs with centromere-localized GFP signal were imaged then centromeric GFP signal was quantified using ImageJ. $\mathrm{p}=0.013$ by $\mathrm{t}$-test. $\mathrm{n}=70$ centromeres per condition. (C) Quantification of centromeric CENP-A signal of cells represented in A. G1 pairs with centromere-localized GFP signal were imaged then centromeric CENP-A signal was quantified using ImageJ. $\mathrm{p}<0.0001$ by $\mathrm{t}$-test. $\mathrm{n}=70$ centromeres per condition. (D) Quantification of centromeric CENP-T signal of cells represented in A. G1 pairs with centromere-localized GFP signal were imaged then centromeric CENP-T signal was quantified using ImageJ. $\mathrm{p}<0.0001$ by $\mathrm{t}$-test. $\mathrm{n}=70$ centromeres per condition. (E and F) Quantification of centromeric CENP-A (E) and CENP-T (F) signals of non-G1 cells from experiment in A. Non-G1 cells were imaged then centromeric CENP-A and CENP-T signal were quantified using ImageJ. $p<0.0001$ by t-test for both $\mathrm{E}$ and $\mathrm{F}$. $\mathrm{n}=70$ centromeres per condition. (G) Representative images of HeLa cells stably expressing Mis18 $\alpha$-GFP. Cells were treated as in A. Cells were stained with antibodies to CENP-A and CENP-T as a centromere markers, and DNA is stained with DAPI. (H, I, J) Centromeric intensity of GFP (H), CENP-A (I), and CENP-T (J) signals from experiment in G. For GFP $(\mathrm{H}) \mathrm{p}<0.0001$ by $\mathrm{t}$-test. CENP-A and CENP-T did not change significantly following RNase cocktail treatment in Mis18 $\alpha$ cells. 


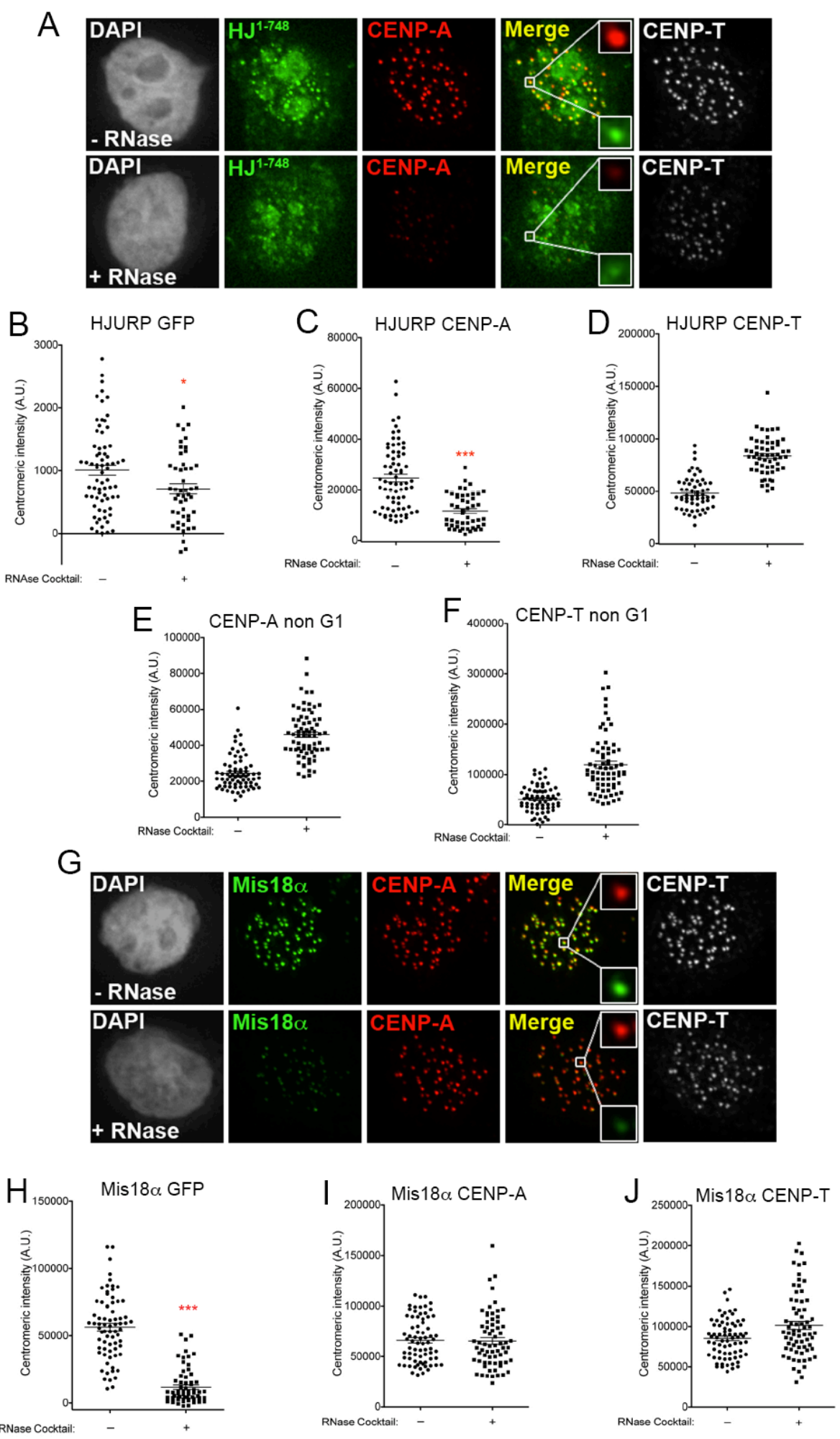


Figure 3-7. Model for SINE28 association with HJURP and its role in CENP-A deposition.

SINE28 is present at the centromere during G1 and throughout the cell cycle. HJURP localizes to centromeres in early G1 bound to pre-nucleosomal CENP-A/H4. SINE28 is likely also associated with HJURP, via its vertebrate conserved domain, at this time. The Mis18 complex recruits HJURP and allows it to deposit new CENP-A at centromeric chromatin. SINE28 and other RNA's play a role in stabilizing both the Mis18 complex and HJURP at centromeres during this time. HJURP deposits new CENP-A and leaves centromeres. SINE28 is required for this successful deposition. 


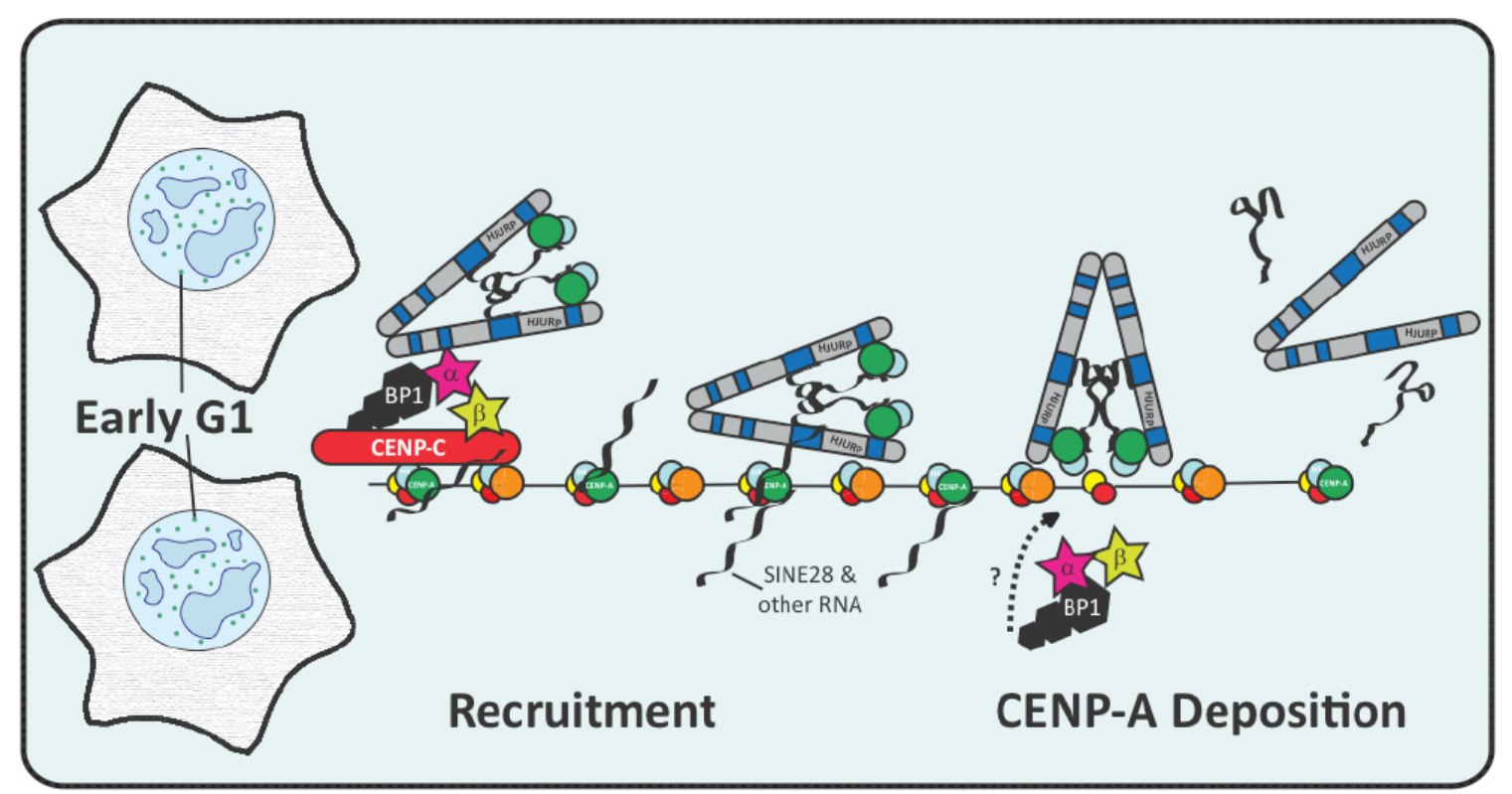




\section{$\underline{\text { Discussion }}$}

We demonstrate the vertebrate conserved domain of HJURP is required for its nucleolar localization and binding to the centromere-enriched ssRNA, SINE28 (Figures 3-1, 3-2, 3-3). Data contributed to this investigation by the O'Neill lab showed that SINE28 is specifically required for new CENP-A deposition at centromeres (Introduction Figure 3-2). We show here that deleting the vertebrate conserved domain of HJURP results in the same loss of CENP-A deposition at centromeres (Figures 3-4, 3-5) as specifically inhibiting SINE28. This led us to predict the mechanism by which SINE28 affects CENP-A deposition may involve its binding to HJURP's vertebrate conserved domain. We find that RNA may also play a more general role in CENP-A deposition through its ability to stabilize CENP-A deposition factors at the centromere during early G1 (Figure 3-6).

We propose a model (Figure 3-7) where the prenucleosomal complex of HJURP and CENP-A/H4 localizes to centromeres in early G1. SINE28 is shown associated with the prenucleosomal complex because the RNA-IP experiments identifying HJURP and CENP-A interaction with SINE28 were done from randomly cycling cells so HJURP and CENP-A are presumably associated with SINE28 outside of early G1 as well, though this needs to be formally tested. The Mis 18 complex recruits HJURP to centromeres. Mis $18 \alpha$ and HJURP are both stabilized at the centromere by RNA while CENP-A deposition occurs. Following CENP-A deposition, HJURP and the Mis18 complex leave centromeres. This work contributes SINE28 RNA binding by HJURP as a new aspect of the CENP-A deposition pathway. 
As this work is currently ongoing, I would like to discuss the experiments that are currently in progress. The main area we are currently improving is determining the specificity of the vertebrate conserved domain for binding to the SINE28 RNA. HJURP and CENP-A both bind to this non-abundant SINE28 robustly in vivo, and this is supportive of a specific interaction. It is possible that the conserved domain of HJURP is required for binding to other RNA's or even to DNA. As such, we are expanding upon the in vitro pull-down studies (Figure 3-3) to look at the specificity of HJURP's conserved domain for SINE28 versus other RNA sequences or a DNA sequence. To strengthen the data that $\mathrm{HJ}^{\Delta 208-350}$ is unable to bind to SINE28 RNA in vivo, I have done RNA-IP purifications using $\mathrm{HJ}^{1-748}$ versus the $\mathrm{HJ}^{\Delta 208-350}$ protein. The cDNA libraries from these experiments are in the process of being sequenced and analyzed. We predict $\mathrm{HJ}^{\Delta 208 \text { - }}$ 350 should lose association with SINE28, which would support the RNA-FISH data for loss of in vivo binding between $\mathrm{HJ}^{\Delta 208-350}$ and SINE28 (Figure 3-2). We also want to investigate the RNA binding capabilities of other CENP-A deposition factors, especially the Mis 18 complex by doing similar in vivo RNA-IP's. Lastly, we are looking at the role of SINE28 RNA in stabilizing the pre-nucleosomal complex.

In Supplemental Figure 3-4 of this work, we characterized the interaction between HJURP and Npm1, another known nucleolar protein and member of the CENP-A prenucleosomal complex (Dunleavy et al., 2009; Foltz et al., 2009; Shuaib et al., 2010). Our results pointed to a requirement for the HJURP conserved domain in Npm1 interaction at the LacO array (Suppl. Figure 3-4 A-C). However, because Npm1 also interacts with CENP-A, and $\mathrm{HJ}^{\Delta 208-350}$ still binds to CENP-A (Suppl. Figure 3-2), we could never observe a black and white loss of Npm1 binding in immunoprecipitation 
experiments. These data are important to consider in the context of the CENP-A deposition loss in the $\mathrm{HJ}^{\Delta 208-350}$ knockdown and rescue experiments since Npm1 is a known member of the CENP-A prenucleosomal complex, though it has no known effect on CENP-A deposition in humans cells (Dunleavy et al., 2009). Npm1 has been documented to bind to RNA as well. Interestingly, Cyclin B/cdc2 inhibits Npm1 RNA binding during mitosis through phosphorylation, and this causes it to leave its usual location in the nucleolus (Okuwaki et al., 2002). One can speculate that this may be part of the pre-nucleosomal assembly pathway where HJURP/CENP-A/H4/Npm1 and perhaps SINE28 are assembled in the nucleoli prior to mitosis then are released in time for their deposition window in early G1. Further characterization of why pre-nucleosomal CENP-A first localizes to the nucleolus (Suppl. Figure 3-5) will help elucidate if nucleolar localization prior to centromeric deposition is a required step for prenucleosomal complex formation and stable CENP-A deposition.

There are a number of possibilities why SINE28 RNA and its interaction with the conserved domain of HJURP is required for CENP-A deposition. SINE28 binding in the pre-nucleosomal complex may provide additional targeting specificity to the centromere by guiding the pre-nucleosomal complex to other nucleic acid targets at the centromere by complementary sequence recognition (Keller and Buhler, 2013). One well-studied example of this are snRNA's and snoRNA's that are required to both recruit and target the splicing machinery, acting as guides and scaffolds at the same time (Matera et al., 2007). Long non-coding RNA's at the centromere may additionally act as a binding site for a smaller "guide" RNA, consistent with SINE28's size. SINE28 could also act strictly as a stabilizing tether at the centromere providing stability for the CENP-A deposition 
machinery there during CENP-A loading, as suggested by RNase treatment reducing both HJURP and Mis18 centromeric stability (Figure 3-6). Lastly, non-coding RNA's have been shown in many organisms, including worms, plants, and mice to be involved in establishing and inheriting a particular epigenetic state (Kiani et al., 2013; Regulski et al., 2013; Shirayama et al., 2012). A similar mechanism could be very important at the centromere in maintaining the correct epigenetic environment for CENP-A deposition, as centromere location is epigenetically specified by its faithful deposition at one locus on each chromosome.

In conclusion, SINE28 is a novel centromere-enriched, short, single-stranded RNA (Introduction Figure 3-1). It specifically associates with both CENP-A and HJURP and is the most abundant RNA element found to bind these proteins (Introduction Figure 3-1). HJURP interacts with SINE28 via its vertebrate conserved domain (Figure 3-2, Figure 3-3). Inhibiting SINE28 RNA interaction with HJURP through addition of a function blocking oligo (Introduction Figure 3-2) or through deleting the vertebrate conserved domain of HJURP causes a loss of CENP-A deposition in human cells (Figure 3-4, Figure 3-5). The small non-coding SINE28 RNA therefore plays an important, previously uncharacterized role in CENP-A deposition. 


\section{$\underline{\text { Supplemental Figures }}$}

Supplemental Figure 3-1. Nucleolar localization sequence prediction.

(A) Coding sequence of human HJURP was input into the nucleolar localization sequence (NoLS) detector, NoD, from the University of Dundee (Scott et al., 2010; Scott et al., 2011). Two predicted nucleolar localization signals were detected in HJURP and are hi-lighted in red. (B) NoLS scores graphed per 20 window position in the protein. Scores above 0.8 (indicated with red shading) are considered NoLS candidate signals. 
NoLS predictions for protein HJURP

(these predictions are based on sequence and Jpred predictions)

2 NoLSs are predicted in this protein:

LQKLRASRRRFQRRMQRLIE (between positions 19 and 38)

IMQNWNSRRRHRYKSRMNKTYCKG (between positions 204 and 227)

Position in full-length protein (NoLSs shown in red):

MLGTLRAMEGEDVEDDQLLQKLRASRRRFQRRMQRLIEKYNQPFEDTPVVQMATLTYETP QGLRIWGGRLIKERNEGEIQCAGNRAGRDVRVTPLPSLASPAVPAPGYCSRISRKSPGDP AKPASSPREWDPLHPSSTDMALVPRNDSLSLQETSSSSFLSSQPFEDDDICNVTISDLYA GMLHSMSRLLSTKPSSIISTKTFIMQNWNSRRRHRYKSRMNKTYCKGARRSQRSSKENFI PCSEPVKGTGALRDCKNVLDVSCRKTGLKLEKAFLEVNRPQIHKLDPSWKERKVTPSKYS SLIYFDSSATYNLDEENRFRTLKWLISPVKIVSRPTIRQGHGENRQREIEIRFDQLHREY CLSPRNQPRRMCLPDSWAMNMYRGGPASPGGLQGLETRRLSLPSSKAKAKSLSEAFENLG KRSLEAGRCLPKSDSSSSLPKTNPTHSATRPQQTSDLHVQGNSSGIFRKSVSPSKTLSVP DKEVPGHGRNRYDEIKEEFDKLHQKYCLKSPGQMTVPLCIGVSTDKASMEVRYQTEGFLG KLNPDPHFQGFQKLPSSPLGCRKSLLGSTAIEAPSSTCVARAITRDGTRDHQFPAKRPRL SEPQGSGRQGNSLGASDGVDNTVRPGDQGSSSQPNSEERGENTSYRMEEKSDFMLEKLE KSV

B

NoLS predictions per residue

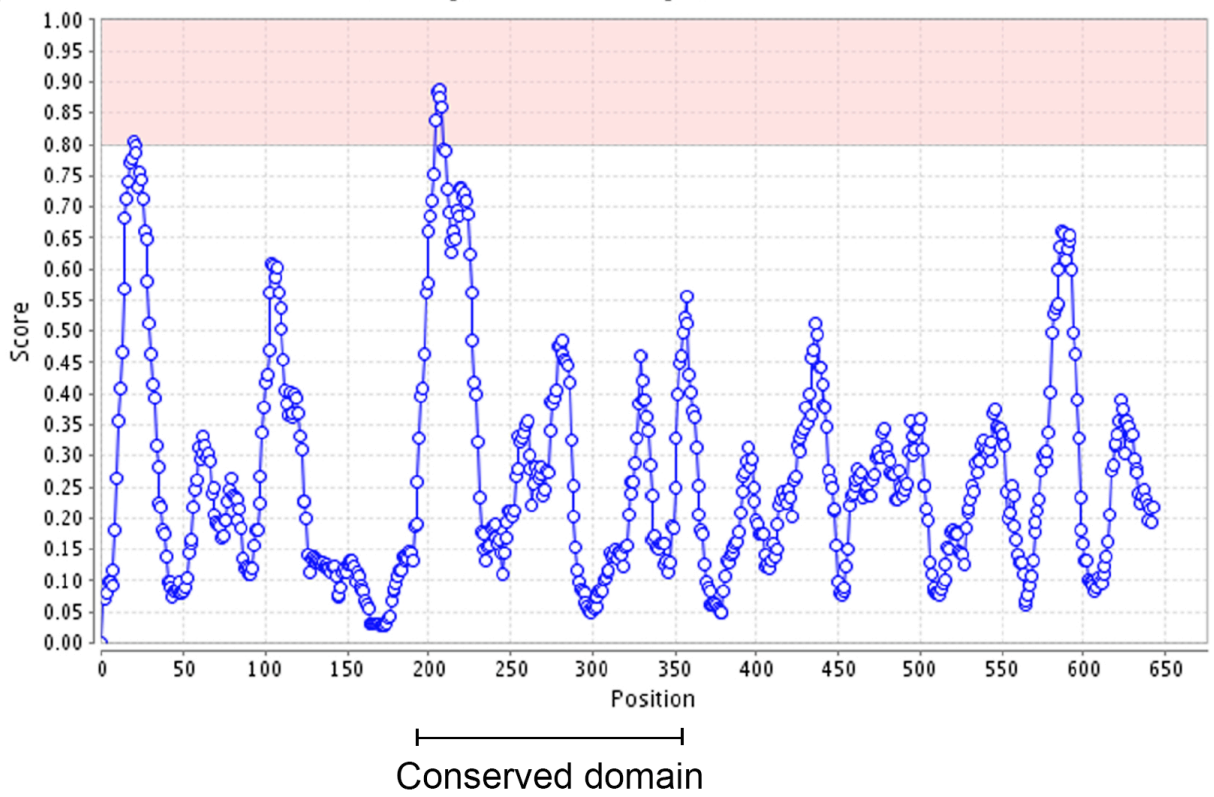


Supplemental Figure 3-2. $H J^{4208-350}$ is able to efficiently bind to CENP-A.

(A) Immunoprecipitation experiment from HEK293T cells transfected for 24 hours with $\mathrm{HJ}^{1-748}$-GFP + mCherry-CENP-A, or $\mathrm{HJ}^{\Delta 208-350}$-GFP + mCherry-CENP-A. IP was done using GFP antibody and immunoprecipitated samples were analyzed by western blot and probed for association with mCherry-CENP-A. Npm1 was also probed as a loading control for the input samples. $\mathrm{N}=4$ replicates. 


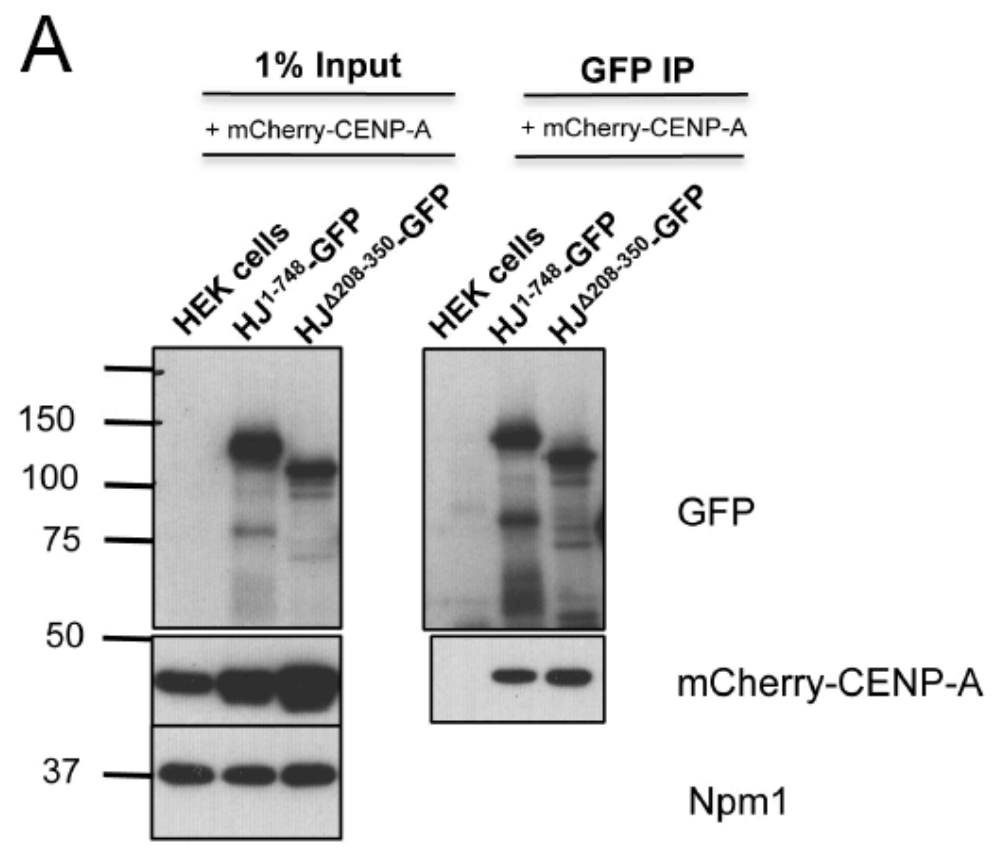


Supplemental Figure 3-3. $H J^{201-348}$ is sufficient for nucleolar localization.

(A) Live cell images of U2OS cells transfected for $24 \mathrm{hrs}$ with GFP-HJ $\mathrm{J}^{201-348}$. Localization patterns are described as nucleolar, speckled, high nuclear, and nucleolar excluded. Images are taken at 100x. Scale bars represent $5 \mu \mathrm{m}$. (B) Quantification of cells in A, graphed as percentage of GFP-HJ $\mathrm{J}^{201-348}$ positive cells with each localization pattern. $\mathrm{n}=30$ cells, $\mathrm{N}=1$ replicate. (C) U2OS cells that were transfected for 24 hrs with GFP$\mathrm{HJ}^{201-348}$ then pre-extracted, fixed, and stained for CENP-A. Localization patterns are described as nucleolar and speckled nucleolar. (D) Quantification of cells in C, graphed as percentage of GFP-HJ ${ }^{201-348}$ positive cells with each localization pattern. $\mathrm{n}=30$ cells, $\mathrm{N}=1$ replicate. 


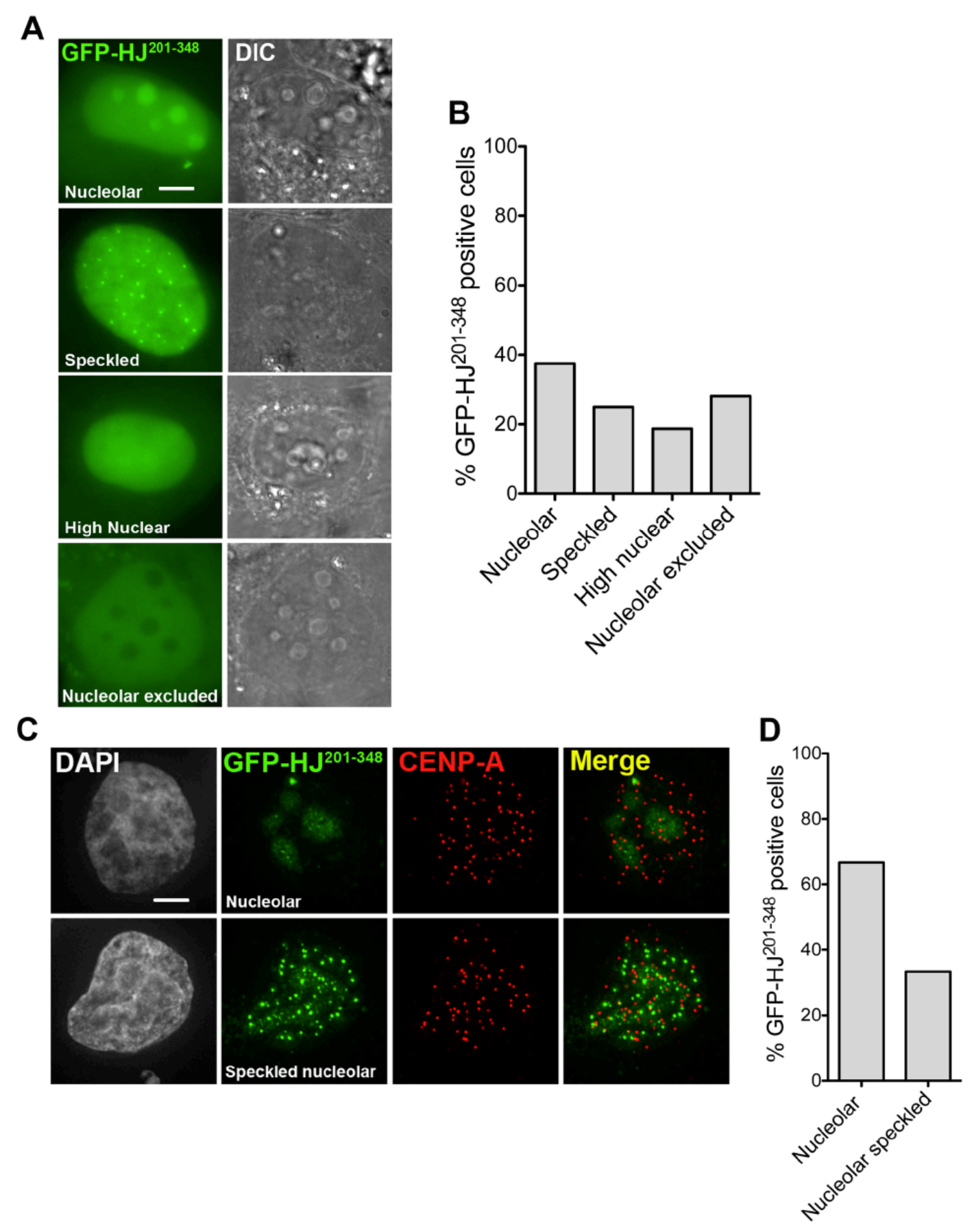


Supplemental Figure 3-4. Npm1 association with HJURP fragments.

(A) Representative images of U2OS-LacO cells transfected for 48 hours with indicated mCherry-LacI-HJURP fragments. Superscript numbers indicate the amino acids present in the targeted fragment. Cells were fixed and stained with an antibody to Npm1 and DAPI to visualize DNA. (B) Quantification of experiment in A. Graph displays the percentage of Npm1 positive arrays for each indicated LacI-HJURP fragment. $\mathrm{n}=30$ arrays counted per condition, $\mathrm{N}=2$ replicates for all conditions except $\mathrm{HJ}^{201-348}$ and $\mathrm{HJ}^{\Delta 208-350}$. Errors bars are standard deviation. (C) Quantification of the intensity of Npm1 at the array minus average nuclear (non-nucleolar) background of Npm1 signal. Error bars are standard deviation. $\mathrm{n}=30$ arrays measured per condition, $\mathrm{N}=2$ replicates for all conditions except $\mathrm{HJ}^{201-348}$ and $\mathrm{HJ}^{\Delta 208-350}$. (D and E) Two replicates of immunoprecipitation experiments from HEK293T cells transfected for 24 hours with $\mathrm{GFP}^{-} \mathrm{HJ}^{1-748}$, GFP-HJ ${ }^{\Delta 208-350}$, GFP-HJ ${ }^{1-348}$, or GFP-HJ ${ }^{1-208}$. GFP fragments were immunoprecipitated with a GFP antibody and bound fractions were analyzed by western blotting. Blots were probed for GFP and Npm1. Two replicates are shown to accurately represent the variability observed in these results. $\mathrm{N}=4$ replicates. 

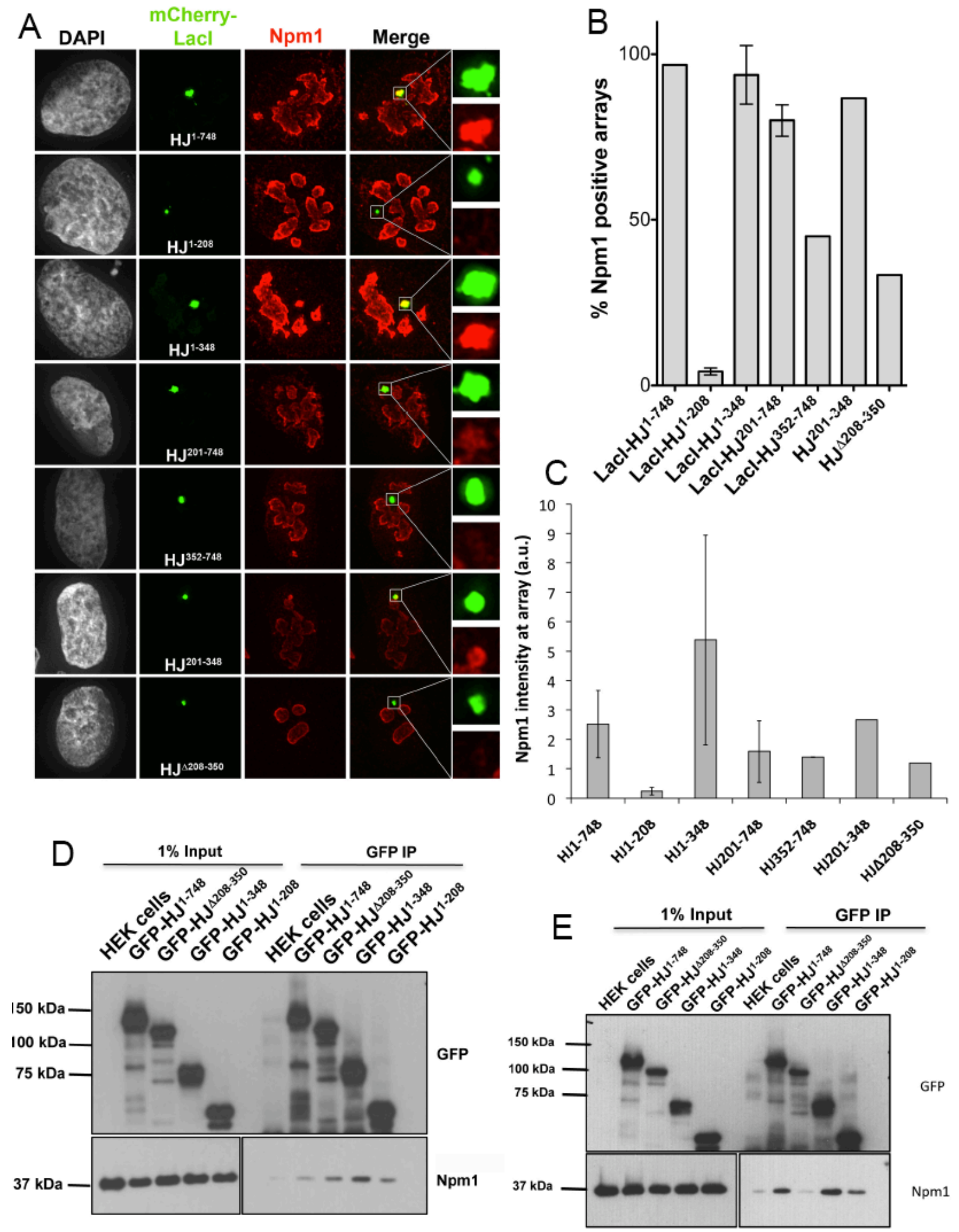
Supplemental Figure 3-5. Newly synthesized CENP-A transits the nucleolus prior to its centromere deposition.

(A) Representative images of HeLa TRex cells stably expressing SNAP-tagged CENP-A. Cells were synchronized at the G1/S boundary using a double thymidine block then treated with BG-block to block the already assembled centromeric CENP-A. Cells were then released into S-phase by addition of deoxycytidine. At timepoints of 0, 3, 5, 7, 9, and 14 hours following release cells were fixed. 1 hour prior to fixation, TMR-Star was added to the media to label the newly synthesized CENP-A. Cells were then fixed and stained with antibodies to CENP-C as a centromere marker and HJURP to mark nucleoli. DNA was stained with DAPI. Insets hi-light that nucleolar SNAP-CENP-A signal accumulates before centromeric SNAP-CENP-A signal. (B) Quantification of the experiment in A. Intensity of SNAP-CENP-A in the nucleoli over general nuclear background was measured at the indicated timepoints. Intensity values were thresholded so only SNAPCENP-A values above the bleed-through intensity (indicated with dotted line) from the HJURP antibody were graphed and analyzed. 5 fields, 8 cells per field for a total of $n=40$ cells per timepoint were measured. $\mathrm{N}=1$ replicate.

SNAP-labeling was done by Ewelina Zasadzinska. MCD performed staining, imaging, and analysis. 


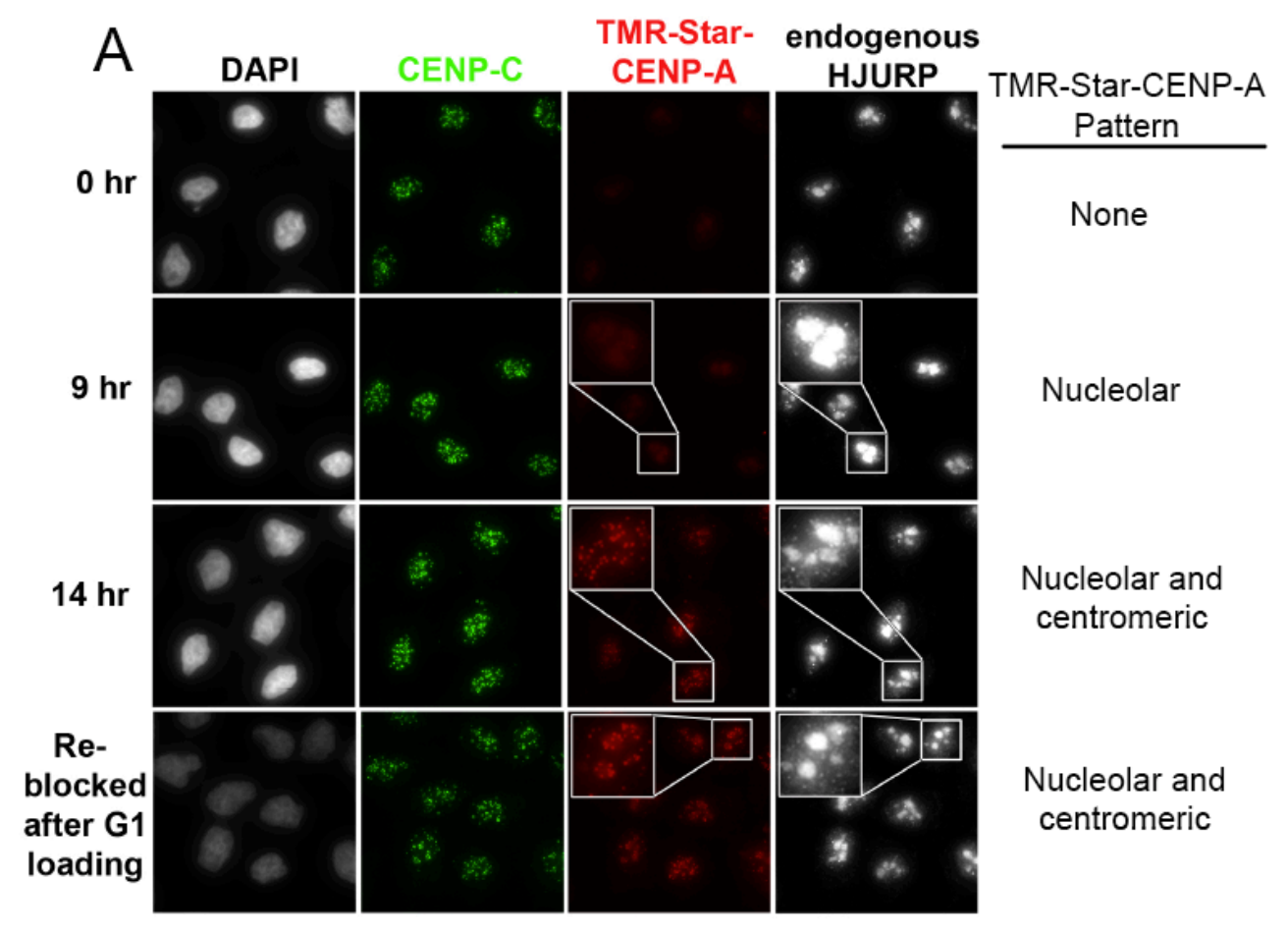

B Nucleolar Snap-CENPA signal

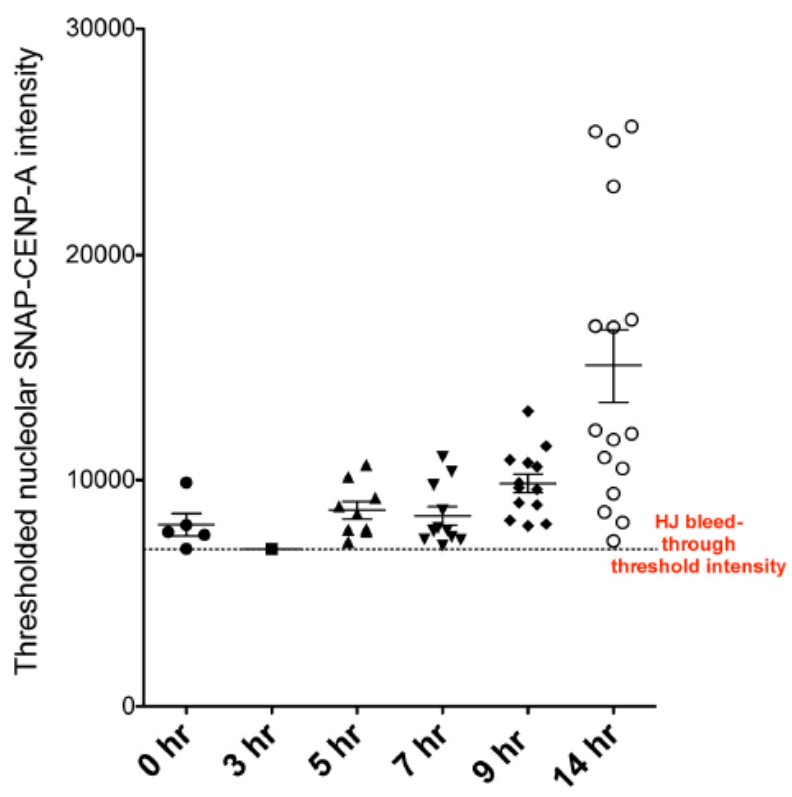

Hours after release from thymidine 
Supplemental Figure 3-6. RNaseA treatment reduces $H^{1-748}$ centromeric recruitment.

(A) Representative images of HeLa TRex cells stably expressing GFP-tagged $\mathrm{HJ}^{1-748}$ or $\mathrm{HJ}^{\Delta 208-350}$. Cells were treated with RNaseA for 20 minutes prior to fixation and staining. Cells were stained with a monoclonal antibody to CENP-A as a centromere marker. (B) Quantification of centromeric GFP signal of cells represented in A. G1 pairs with centromere-localized GFP signal were imaged then centromeric GFP signal was quantified using ImageJ. For $\mathrm{HJ}^{1-748}$ and $\mathrm{HJ}^{\Delta 208-350} \mathrm{n}>70$ centromeres per condition, $\mathrm{N}=2$ replicates. Blue lines indicate the means and standard error means for each condition. Asterisks indicate statistically significantly different $(\mathrm{p}<0.0001)$ from no RNaseA treatment by Kruskal-Wallis test followed by Dunn's multiple comparison test. (C) Centromeric CENP-A intensities (endogenous CENP-A) from the experiment graphed in B. Asterisks indicate group is statistically different from no RNase treatment, $p<0.0001$ by Kruskal-Wallis test followed by Dunn's multiple comparison test. 

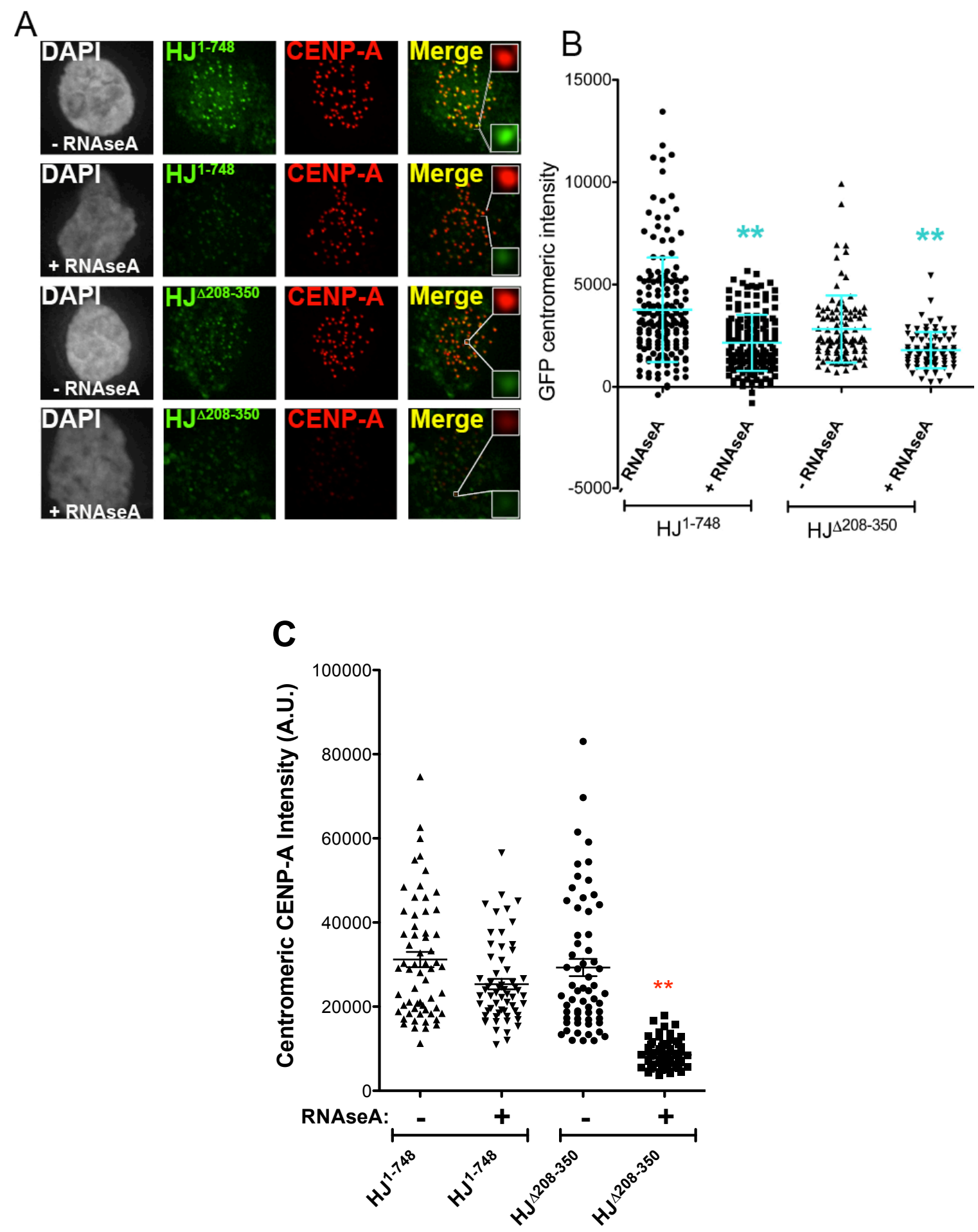


\section{Materials and Methods}

RNase treatments

HeLa-TRex cell lines stably expressing Mis18 $\alpha-G F P, \mathrm{HJ}^{1-748}-\mathrm{GFP}$, or $\mathrm{HJ}^{\Delta 208-350}{ }_{-\mathrm{GFP}}$ were either treated with RNase cocktail containing RNaseA+RNaseT1 or RNaseA alone following this same protocol. Cells were plated at 60,000 cells/well in a $24-w e l l$ plate on polylysine-coated coverslips and allowed to sit down overnight. Cells were washed 1x with PBS for 30 seconds at RT. Cells were pre-extracted in CSK buffer (10mM PIPES pH 7.0, 100mM NaCl, 300mM sucrose, $3 \mathrm{mM} \mathrm{MgCl}_{2}, 0.5 \mathrm{mM}$ PMSF, $10 \mu \mathrm{g} / \mathrm{mL}$ LPC) + $0.05 \%$ Triton-X for 4 minutes. Wash $1 \mathrm{x}$ with CSK buffer for $3 \mathrm{~min}$ at room temperature. Rinse with PBS with 2 minutes. Add 1mg/mL RNaseA alone (Roche 10109169001) or $1 \mu \mathrm{l}$ per 24 well plate for RNase cocktail (RNaseA+RNaseT1) (Ambion AM2286) in $300 \mu \mathrm{L}$ PBS per well of 24-well plate for 20 minutes. Wash with PBS 2 minutes, 2x at RT. Proceeded with standard fixation and staining procedure described in immunocytochemistry section.

\section{SNAP labeling}

HeLa-TRex cells stably expressing SNAP-tagged CENP-A (Jansen et al., 2007) were synchronized using a double thymidine block and release $(16 \mathrm{hr} 20 \mathrm{mM}$ thymidine, $9 \mathrm{hr}$ $24 \mu \mathrm{M}$ deoxycytidine, $16 \mathrm{hr} 20 \mathrm{mM}$ thymidine). Prior to $2^{\text {nd }}$ release into deoxycytidine, the preassembled CENP-A was blocked with $10 \mu \mathrm{M} \mathrm{O}^{6}$-BG (BG-block; Covalys) for 30 min at $37^{\circ} \mathrm{C}$ followed by a PBS wash and three washes with DMEM over $30 \mathrm{~min}$. Cells were then released with $24 \mu \mathrm{M}$ deoxycytidine. New CENP-A was labeled 1 hour prior to each timepoint through S-phase and into the following G1 (1, 3, 5, 7, 9, 14 hrs, and re- 
blocked in thymidine) with $2 \mu \mathrm{M}$ TMR-Star (Covalys) in complete growth medium for 30 min at $37^{\circ} \mathrm{C}$. After 30 minutes of labeling, cells were washed one time in PBS, then $2 x$ in DMEM, then incubated for 30 min in DMEM to dilute away unbound TMR-Star, and washed with PBS prior to standard fixation and staining procedure.

\section{Knockdown and rescue experiments}

For both of the knockdown and rescue experiments shown, (overall CENP-A levels vs. new CENP-A loading), HeLa-TRex cells stably expressing either $\mathrm{HJ}^{1-748}$-GFP, $\mathrm{HJ}^{\Delta 208-350}$ GFP, or un-rescued parental lines were used. To deplete endogenous HJURP, cells were plated at a density of 100,000 cells per well (6-well format). After cells sat down overnight, for overall CENP-A level experiment, cells were treated with 20nM HJURP 5' UTR (Custom duplex siRNA Thermo Scientific 5' GGGUUGGCGCUUGGGUACUUU 3') + 20nM HJURP 3' UTR siRNA (Custom duplex siRNA Thermo Scientific 5' GAGAUAACCUCGAGUUCUUUU 3') or 40nM Negative Control 2 Silencer Select siRNA (LifeTechnologies, Catalog \# 4390846). siRNA treatments were done in Optimem using RNAiMAX as lipofection reagent. After 24 hours, 1/3 of the plating volume DMEM with $10 \%$ heat inactivated FBS $+5 \%$ Pen/Strep was added and cells were left an additional 24 hours to complete depletion. For the new CENP-A loading experiment, cells were co-transfected with siRNA as above and an mCherry-CENP-A expressing plasmid. $1 \mu \mathrm{g}$ plasmid, same amount of siRNA listed above, and $2 \mu \mathrm{L}$ of Lipofectamine2000 at the lipofection reagent were used per well of 6-well plate. Cells were left in serum-free media until 24 hours prior to fixation at which point DMEM + $10 \% \mathrm{FBS}+1 \% \mathrm{Pen} / \mathrm{Strep}$ was added to replace Optimem for an additional 24 hours before harvesting. 


\section{In vitro pull-down}

50 ng biotinylated SINE28 single-stranded sense and anti-sense RNA (obtained from O’Neill lab) was incubated at a 1:1 molar ratio with recombinant MBP-HJ, MBP-HJ ${ }^{\triangle 208-}$ 350, MBP-HJ ${ }^{1-348}$, or MBP alone. For the MBP-HJURP ${ }^{1-348}$ condition, recombinant CENP-A/H4 heterotetramer was also added in one condition. CENP-A/H4 heterotetramer was also incubated alone with the SINE28 RNA. Components were mixed with RNA for 2 hours at room temperature on a rotator. The components were mixed in $300 \mu \mathrm{L}$ of the following reaction buffer: $50 \mathrm{mM}$ tris ph $7.5,250 \mathrm{mM} \mathrm{NaCl}, 20 \mathrm{mM} \mathrm{Mgcl} 2,0.5 \% \mathrm{~Np} 40$, $1 \mathrm{mM}$ DTT, and $10 \%$ glycerol. $4 \mu \mathrm{L}$ streptavidin-coated beads, which were pre-blocked in $0.2 \mathrm{mg} / \mathrm{mL}$ BSA in reaction buffer, were added for 40 minutes with rotation at room temperature. Beads were washed $5 \mathrm{x}$ in reaction buffer then resuspended in $15 \mu \mathrm{L}$ of sample buffer.

\section{Western Blotting}

For HJURP depletion western blot analysis, cells were harvested forty-eight hours after siRNA treatment with PBS + 3 mM EDTA, counted and whole-cell lysates were made in SDS-PAGE sample buffer. $15 \mu \mathrm{L}$ lysate containing $1 \times 10^{5}$ cells per lane were separated on $10 \%$ SDS-PAGE gel and transferred to nitrocellulose. Blots were blocked in $7 \%$ milk for 2 hours then incubated in primary anti-HJURP (\#3399) or anti-tubulin (AA2) antibody overnight at $4^{\circ} \mathrm{C}$ and in secondary 1:10,000 (Jackson Laboratories) for 1 hour RT. SuperSignal West Pico ECL reagent was used for chemiluminescence (Pierce 34077). For MBP western blotting, membrane was incubated in MBP antibody at 1:10,000 overnight at $4^{\circ}$ (NEB E8038S). GFP (3404) antibody was used at 1:1000 and Npm1 antibody was used at 1:5000 overnight at $4^{\circ}$ for western blot. CENP-A antibody was used 
at 1:1000 overnight at $4^{\circ}$ for western blotting.

\section{Cell culture, transfections, and immunocytochemistry}

HeLa or U2OS-LacO cells were plated to poly-lysine coated coverslips at $1 \times 10^{5}$ cells per well in 6 well, $0.6 \times 10^{5}$ cells per well in 24 -well plate, or $1 \times 10^{6}$ cells for a $10 \mathrm{~cm}^{2}$ plate. Cells were transfected in Optimem 24 hours later with $0.2-0.25$ ug plasmid DNA (24well plate), $1 \mathrm{ug}$ (6-well plate), or $5.8 \mathrm{ug}\left(10 \mathrm{~cm}^{2}\right.$ dish) using $0.4 \mathrm{uL}$ (24-well plate), $2 \mathrm{uL}$ (6-well plate), or $11.6 \mathrm{uL}\left(10 \mathrm{~cm}^{2}\right.$ dish) Lipofectamine ${ }^{\mathrm{TM}} 2000$ (LifeTechnologies 11668 027). Cells were left in Optimem + transfection complexes for 9 hours then media was changed to DMEM + 10\% FBS, 5\% Pen/Strep. HEK293T cells were transfected in serum-free, Pen/Strep-free DMEM using 4ug DNA, 30 uL Polyfect (Qiagen 301105) per $6 \mathrm{~cm}^{2}$ dish.

Live-cell imaging was conducted on the U2OS cell line in Leibovitz L-15 media including 10\% FBS following $24 \mathrm{hrs}$ of transfection. Images were collected on a Deltavision Microscope equipped with a Weatherstation environmental chamber maintained at $37^{\circ} \mathrm{C}$.

For fixation, U2OS-LacO and HeLa-TRex cells were pre-extracted with $0.1 \%$ Triton-X in PHEM Buffer for 3 minutes (RNase-treated experiments cells were preextracted differently, see that section), fixed with $4 \%$ paraformaldehyde in PBS for 10 minutes, and then quenched by addition of $100 \mathrm{mM}$ Tris- $\mathrm{HCl}, \mathrm{pH} 7.5$ for another 5 minutes at room temperature. Cells were blocked in 2\% FBS, $2 \%$ BSA in $0.1 \%$ TritionPBS. Centromeres were visualized with a rabbit polyclonal anti-CENP-T antibody 1:2000 (\#3408), a monoclonal CENP-C antibody (CX-191-1) at 1:1000 dilution, or a monoclonal CENP-A antibody at 1:1000 dilution (Abcam, ab13939). Endogenous 
HJURP was stained using rabbit polyclonal antibody (\#3399) at 1:1000. DNA was stained with 4',6-diamidino-2-phenylindole (DAPI, $0.2 \mathrm{mg} / \mathrm{ml}$ ). Donkey-anti-rabbit Cy5conjugated (Jackson Laboratories, \#111175003) or Cy-3 or FITC-conjugated goat antimouse, or secondary antibodies were used for detection and coverslips were mounted with 4 uL ProlongGold (LifeTechnologies).

All micrograph images were collected using either a $60 \mathrm{x}$ or $100 \mathrm{x}$ oil-immersion Olympus objective lens (numerical aperture $=1.40$ ) on a DeltaVision deconvolution microscope using a Photometrics CoolSNAP $\mathrm{HQ}^{2}$ camera. Acquisition software used was SoftWoRX from Applied Precision. Fixed cell images were deconvolved and presented as stacked images. All representative images within cell lines within figures were collected with identical exposure times and scaled equally. Intensities in live-cell and fixed images were analyzed using ImageJ. Details of each analysis method are provided in appropriate figure legends. General formula used for any centromeric intensity analysis is $\mathrm{IL}=$ Intensity large circle, $\mathrm{IS}=$ Intensity small circle, $\mathrm{AL}=$ Area large circle, $\mathrm{AS}=$ Area small circle. Bkg corrected centromere intensity $=\mathrm{IL}-(((\mathrm{IS}-\mathrm{IL}) * \mathrm{AL}) /(\mathrm{AS}-\mathrm{AL}))$

\section{Immunoprecipitations}

HEK293T cells were plated to $90 \%$ confluency in $6 \mathrm{~cm}^{2}$ dishes. After 24 hours, cells were transfected using Polyfect (Qiagen) as described above. For mitotically arrested population, $0.1 \mathrm{ug} / \mathrm{mL}$ nocodazole was added to the media for 12 hours. After 24 hours, cells were harvested on ice using PBS $+3 \mathrm{mM}$ EDTA. Cells were spun down then washed $1 \mathrm{x}$ with PBS. Cells were lysed for 10 minutes on ice in $1 \mathrm{~mL}$ of RIPA buffer with occasional vortexing $(150 \mathrm{mM} \mathrm{NaCl}, 1 \% \mathrm{NP}-40,0.3 \%$ deoxycholate, $0.15 \%$ SDS, $50 \mathrm{mM}$ Tris-HCl pH 7.5, 1mM EDTA, 10\% glycerol, 1x Roche protease inhibitors, 200uM NaV, 
$0.5 \mathrm{mM}$ PMSF, $5 \mathrm{mM} \mathrm{NaF}, 50 \mathrm{mM}$ beta-glycerophosphate). Lysates were sonicated $2 \times 10$ cycles $30 \mathrm{sec}$ on/30sec off using Biorupter. Lysates were spun down max speed then the full $1 \mathrm{~mL}$ supernatent was pre-cleared with $10 \mathrm{uL}$ Protein A agarose beads for 1 hour on ice. Beads were spun out and supernatents were transferred to a fresh tube and 1uL GFP antibody (3404) or $0.2 \mathrm{uL}$ of IgG was added overnight at $4^{\circ}$ with rotation. The next day, 8uL Protein A Dynabeads (LifeTechnologies 10001D) were added for 1 hour on ice. Beads were washed 1 $\mathrm{x}$ with RIPA buffer and 3x with PBST. Beads were resuspended in sample buffer.

\section{Antibodies}

1. CENP-A mouse monoclonal (Abcam 13939), 1:1000, immunofluorescence

2. CENP-T rabbit polyclonal (\#3408), 1:2000, immunofluorescence

3. GFP rabbit polyclonal (\#3404), 1:1000, immunoblotting

4. Npm1 mouse monoclonal, 1:10,000, immunoblotting

5. HJURP rabbit polyclonal (\#3399), 1:1000, immunofluorescence

6. MBP-HRP mouse monoclonal (NEB E8038S), 1:8000, immunoblotting

7. CENP-C mouse monoclonal (CX-191-1) 1:1000, immunofluorescence 


\section{$\underline{\text { Acknowledgements }}$}

We would like to thank the Rachel O’Neill and Chu Zhang, for their collaboration and helpful discussion on this project. We thank them for performing the RNA FISH staining and imaging, and for providing the biotinylated RNA for the in vitro pull-down assays. We also thank Chu Zhang for providing the two introduction figures in this work. 
CHAPTER IV: INTERACTION OF HJURP WITH THE CONDENSIN II COMPLEX AND ITS ROLE IN CENP-A DEPOSITION 


\section{$\underline{\text { Abstract }}$}

Centromeric chromatin is required for kinetochore assembly during mitosis and therefore accurate chromosome segregation. The H3-specific variant CENP-A is the defining feature of centromeric chromatin. In human cells, CENP-A deposition occurs at a unique time during the cell cycle. The CENP-A deposition machinery localizes to centromeres just following mitotic exit, and CENP-A deposition proceeds in early G1. The mechanism by which CENP-A is deposited onto a condensed chromatin template is not understood. We show that when the CENP-A chaperone and assembly factor HJURP is targeted to a $\mathrm{LacO}$ array, chromatin decondensation results at that site. We demonstrate this decondensation triggers recruitment of and interaction with the condensin II complex. Condensin II recruitment is required to temper HJURP decondensation and its depletion results in reduced HJURP recruitment to centromeres. Finally, we show condensin II function at the centromere is required for new CENP-A deposition. These data identify a novel condensin II complex interaction with HJURP, as well as a novel role for condensin II in CENP-A deposition in human cells. 


\section{Introduction}

Unlike canonical nucleosome assembly, new CENP-A deposition in metazoans is uncoupled from centromeric chromatin replication. Instead, CENP-A is re-loaded at the centromere by its chaperone HJURP in early G1 just after the cell exits mitosis (Dunleavy et al., 2009; Foltz et al., 2009; Jansen et al., 2007). There is some evidence H3.3 may act as a placeholder for CENP-A from S-phase until G1, but how this is exchanged for CENP-A in G1 is not known (Dunleavy et al., 2011). Replication independent CENP-A assembly presents the additional challenge of condensed postmitotic chromatin for use as a template. How the CENP-A deposition machinery accesses this condensed centromeric chromatin is unknown. So while it is understood when CENP-A is loaded at the centromere and what major players are involved in getting it there, relatively little is known about the mechanism of assembly - if the chromatin is remodeled, if $\mathrm{H} 3.3$ is removed, or what role the epigenetic landscape at the centromere plays.

There is precedence for the CENP-A deposition machinery to interact with chromatin remodeling proteins suggesting that chromatin remodeling at the centromere is important for deposition. HJURP, the Mis18 complex members, and CENP-A all copurify with RbAp46/48 in humans and yeast (Dunleavy et al., 2009; Foltz et al., 2009; Fujita et al., 2007; Hayashi et al., 2004; Shuaib et al., 2010). RbAp46/48 are known members of several chromatin remodeling complexes, including CAF1 and NuRD, both of which harbor ATP-dependent nucleosome remodeling activity (Loyola and Almouzni, 2004). Depleting RbAp46/48 causes a reduction in CENP-A loading (Fujita et al., 2007; Hayashi et al., 2004). In addition to RbAp46/48, HJURP and CENP-A co-purify with 
Npm1, another histone chaperone (Dunleavy et al., 2009; Foltz et al., 2009; Shuaib et al., 2010). Lastly, HJURP co-purifies with DNA helicases Tip49a/Tip49b, which both harbor ATPase activity (Shuaib et al., 2010). Why these chromatin remodeling proteins all copurify with the CENP-A deposition machinery is not currently understood and suggests that chromatin remodeling may be a critical step in CENP-A deposition at the centromere.

Along with the CENP-A chaperone HJURP, the Mis18 complex is also required for CENP-A loading in human cells (Fujita et al., 2007). The Mis18 complex may epigenetically prime centromeric chromatin in some way to make it compliant for HJURP recruitment and CENP-A deposition. This priming mechanism is not well understood but may create a chromatin landscape that will be amenable to the CENP-A deposition machinery. There is evidence the Mis18 complex may influence histone acetylation at centromeric repeats (Fujita et al., 2007; Hayashi et al., 2004). Mis18 $\alpha$ has also been shown to interact with DNMT3A and DNMT3B, and eliminating this interaction by depleting Mis $18 \alpha$ led to a reduction in methylation of centromeric chromatin and decreased centromeric CENP-A levels (Gopalakrishnan et al., 2009; Kim et al., 2012). However, DNMT3 knockout mice are viable, so its role may be an auxiliary one. Mouse embryonic fibroblasts lacking Mis18 $\alpha$ have a chromatin condensation defect in prometaphase providing evidence that Mis $18 \alpha$ may be able to condense chromatin in some way (Kim et al., 2012). All these findings are consistent with the Mis 18 complex preparing the centromeric chromatin for accessibility by the CENP-A deposition machinery. 
Lastly, chromatin remodeling may be important for CENP-A deposition because of the highly condensed post-mitotic chromatin onto which CENP-A is deposited. As cells transition from interphase into mitosis, chromosomes compact during prophase on average 2-3 fold, based on data from multiple approaches including FISH, live cell imaging, and FRET to measure the distances between histone molecules (Lleres et al., 2009; Martin and Cardoso, 2010; Vagnarelli, 2012). Chromosome condensation is triggered by the activation of CyclinB1-Cdk1 when it enters the nucleus in prophase (Gavet and Pines, 2010a, b). Chromatin condensation during prophase is mainly driven by a combination of histone tail modifications and through the condensation activity of the condensin I and II complexes in human cells, though there is evidence a small degree of chromatin condensation still occurs in the absence of the condensin complexes (Vagnarelli et al., 2006). Histone tail modifications play a role in encouraging compaction by increasing inter- and intra-nucleosomal interactions. Phosphorylation on serine 10 of histone $\mathrm{H} 3$ by Aurora $\mathrm{B}$ during mitosis was recently shown to induce chromatin compaction by recruiting the histone deacetylase Hst2p. Hst2p deacetylates histone $\mathrm{H} 4$ on lysine 16 allowing its tail to interact with neighboring nucleosomes, which leads to chromatin compaction (Wilkins et al., 2014). Additionally, phosphorylation of the CENP-A N-terminal tail on serine 16 and 18 causes inter and intramolecular salt bridge formation on the CENP-A tails, which could influence the local chromatin compaction environment at the centromere during mitosis and interphase (Bailey et al., 2013).

In addition to histone tail modifications, the condensin complexes play the fundamental role in inducing this chromatin condensation during mitosis. Many 
organisms, including humans, contain two condensin complexes. The two complexes both share the SMC2 and SMC4 (Structural Maintenance of Chromosomes) subunits, which are chromosomal ATPases (Hirano and Hirano, 2006). Condensin I and II each contain three complex-specific subunits, CAP-D2, CAP-G, CAP-H, and CAP-D3, CAPG2, CAP-H2 for condensin I and II, respectively (Hirano, 2012). CAP-H and CAP-H2 are the kleisin subunits of the condensin I and condensin II complexes, respectively. Wherever investigated, these kleisin subunits have been found to function together with the SMC proteins. The kleisins have conserved $\mathrm{N}$ and $\mathrm{C}$-terminal globular domains joined by a flexible linker, and they form a ring-like structure by binding to the SMC proteins on their N and C-terminal ends (Schleiffer et al., 2003). Condensin II is localized in the nucleus throughout the cell cycle whereas condensin I only gains access upon nuclear envelope breakdown in prometaphase. Condensin II is largely responsible for the axial shortening of mitotic chromosomes, and it works alongside condensin I, which is responsible for lateral compaction of mitotic chromosomes (Ono et al., 2003).

Interestingly, in humans, C. elegans, Xenopus, and Drosophila, the Condensin II complex specifically enriches at centromeric, CENP-A-containing chromatin during mitosis, whereas the condensin I complex is absent from these regions (Ono et al., 2004; Savvidou et al., 2005; Shintomi and Hirano, 2011; Stear and Roth, 2002). This enrichment of condensin II at the centromere in human cells requires Aurora B (Ono et al., 2004). Additionally, condensins have previously been shown to affect CENP-A deposition in both yeast and humans. Depleting the common condensin subunits, SMC2 and SMC4, results in a reduction in CENP-A/Cse4 loading in yeast and human cells (Samoshkin et al., 2009; Yong-Gonzalez et al., 2007). Depleting specifically the 
condensin II complex in Xenopus egg extracts also results in a loss of CENP-A loading (Bernad et al., 2011). This along with the enrichment of condensin II at CENP-A chromatin suggests that specifically the condensin II complex may have a unique contribution at centromeric chromatin.

Here we demonstrate the CENP-A chaperone HJURP induces chromatin decondensation when targeted to a $\mathrm{LacO} / \mathrm{TRE}$ array in $\mathrm{U} 2 \mathrm{OS}$ cells. We find the same region of HJURP that induces this decondensation is sufficient to specifically recruit the condensin II complex and that its recruitment is necessary for controlling the extent of decondensation. Preliminary evidence suggests Mis $18 \alpha$ may be involved in tempering the ability of HJURP to decondense chromatin. We also demonstrate that condensin II and HJURP co-purify in human cells. Lastly, we show depletion of the condensin II complex reduces HJURP recruitment to centromeres and subsequently results in a reduction of new CENP-A loading at the human centromere. 


\section{$\underline{\text { Results }}$}

\section{HJURP induces chromatin decondensation}

In previous studies, we demonstrated targeting the CENP-A chaperone HJURP to a non-centromeric, LacO/TRE array induced CENP-A chromatin establishment and de novo kinetochore formation at that site (Barnhart et al., 2011). In addition to recruiting and depositing CENP-A, HJURP affected the chromatin compaction state at the LacO/TRE array as compared to targeting LacI alone (Figure 4-1 A). To investigate this further, we targeted fragments containing separate portions of HJURP to the LacO/TRE array to narrow down which region of the protein was responsible (Figure 4-1 B). In order to quantify the chromatin compaction status at the array, the longest axis of each array imaged was set as a ratio to its shortest axis (Figure 4-1 C). A perfectly round shape would give a value of 1 whereas an elongated shape would increase this length to width ratio. All fragments of HJURP containing amino acids 201-748 were sufficient to significantly increase the length to width ratio of the array as compared to LacI (Figure 4-

1 C). The minimal fragment required to induce decondensation was $\mathrm{HJ}^{352-748}$. The conserved CENP-A binding Scm3 domain of HJURP $\left(\mathrm{HJ}^{1-208}\right)$ was not sufficient to decondense the array suggesting this property of HJURP may be unique to vertebrates, which contain an expanded C-terminus of HJURP (Sanchez-Pulido et al., 2009). Targeting the activator protein VP-16 to LacO arrays has previously been shown to induce a chromatin morphology change (Rafalska-Metcalf et al., 2010). VP-16 induces a characteristic rosette shape of local decondensation associated with transcription initiation (Tumbar et al., 1999). We therefore used this as a positive control for chromatin 
condensation change in our assay (Figure 4-1 B, C). While VP-16 did produce a localized change in chromatin morphology at the LacO array as previously observed, HJURP dramatically extended the LacO arrays, instead of just causing a local decondensation event like VP-16. This is hi-lighted by the increased length to width ratios induced by HJURP versus VP-16 (Figure 4-1 C).

Previous studies of DDB2, which decondenses chromatin when targeted to a LacO/TRE array, found that histone occupancy was reduced at the expanded arrays (Luijsterburg et al., 2012). To test if this was the case when LacI-HJ was targeted we performed ChIP using pan-H3 antibodies and probed for its association with the LacO array. We did not observe a deficit in $\mathrm{H} 3$ at the $\mathrm{LacO}$ array when LacI-HJ ${ }^{1-748}$ was targeted, in fact we consistently observed an increase in H3 signal (Supp. Figure 4-1 A). One explanation for the increased $\mathrm{H} 3$ signal is the decondensation induced by HJURP allowed increased accessibility to shearing during sonication of the samples, thus increasing the $\mathrm{H} 3$ accessibility. Another explanation for the observed increase in $\mathrm{H} 3$ signal is establishment of CENP-A chromatin at the LacO array when HJURP is targeted may also result in $\mathrm{H} 3$ deposition. $\mathrm{H} 3$ nucleosomes are interspersed with CENP-A nucleosomes at endogenous centromeres, and it is possible this arrangement is important for stable CENP-A deposition (Blower et al., 2002; Sullivan and Karpen, 2004). Consistent with this, CENP-T was also recruited to these arrays, and CENP-T is known to purify more closely with H3 chromatin at the centromere (Barnhart et al., 2011; Hori et al., 2008a).

We next wanted to determine if the array decondensation induced by HJURP binding at the $\mathrm{LacO} / \mathrm{TRE}$ array was reversible. A readily reversible re-condensation of 
the array upon HJURP removal would be consistent with a direct role of HJURP, or a protein closely associated with it, in the decondensation event. To test this, live cell imaging was performed on U2OS-LacO cells expressing mCherry-LacI-HJ ${ }^{1-348}$, which is sufficient to decondense the array (Figure 4-1 C), and GFP-TetR, an independent array marker. After filming for 5 minutes, media containing IPTG was washed into the chamber to disrupt the mCherry-LacI-HJ ${ }^{1-348}$ binding at the LacO array. By 6 minutes, mCherry-LacI-HJ ${ }^{1-348}$ was no longer visible at the array in IPTG treated cells. Using the GFP-TetR marker to follow the array compaction status, the cells were imaged for 84 minutes. Immediately upon mCherry-LacI-HJ ${ }^{1-348}$ removal by IPTG, the array began to re-condense (Figure 4-1 D). By 84 minutes, decondensed arrays had constricted on average to $35 \%$ of their initial length (Figure 4-1 E). We additionally tested if known, closely bound proteins to HJURP possess any decondensation activity. Npm1 is associated with the HJURP-CENP-A-H4 pre-nucleosomal complex and also localizes to centromeres with HJURP during early G1 (Dunleavy et al., 2009; Foltz et al., 2009). Unlike HJURP, targeting mCherry-LacI-Npm1 to the array did not result in any chromatin decondensation (Supp. Figure 4-1 B, C). We cannot yet rule out if another closely bound or recruited protein is involved in this process. Together these data indicate that HJURP targeting to chromatin in human cells results in a decondensed chromatin state that is readily reversible upon HJURP removal from the site. 
Figure 4-1: HJURP induces chromatin decondensation at LacO array

(A) Representative images of U2OS-LacO cells transfected for 48 hours with mCherryLacI or mCherry-LacI-HJ ${ }^{1-748}$. Centromeres are marked using a monoclonal antibody to CENP-A. Scale bar represents $5 \mu \mathrm{m}$. (B) Representative images of indicated fragments of HJURP tagged with mCherry-LacI and transfected into U2OS-LacO cells for 48 hours. mCherry-LacI-VP-16 was used as a positive control for decondensation. Centromeres are stained as in A. (C) Quantification of array decondensation represented in A and B. The decondensation is quantified as a ratio of the longest length of each array to its widest width. $\mathrm{n} \geq 30$ arrays per condition, $\mathrm{N}=2$ biological replicates. Asterisks represent conditions statistically different as compared to LacI alone, $\mathrm{p} \leq 0.0001$ by Kruskal-Wallis test followed by Dunn's Multiple Comparison test. (D) Representative live cell images of U2OS-LacO cells co-transfected for 24 hours with mCherry-LacI-HJ ${ }^{1-348}$ and GFP-TetR. At 5 minutes of imaging, $15 \mathrm{mM}$ IPTG was washed into media to remove mCherry-LacI$\mathrm{HJ}^{1-348}$. Mock treatment was just media alone. Cells were imaged every 6 minutes for 84 minutes. Scale bar represents $5 \mu \mathrm{m}$. (E) Quantification of experiment in D. Graph represents $n=10$ arrays each for mock and 15 mM IPTG treatment. Array length was measured at each timepoint and graphed as the percentage of the initial length for each timepoint. Error bars represent the standard deviation between the 10 images for each timepoint. 

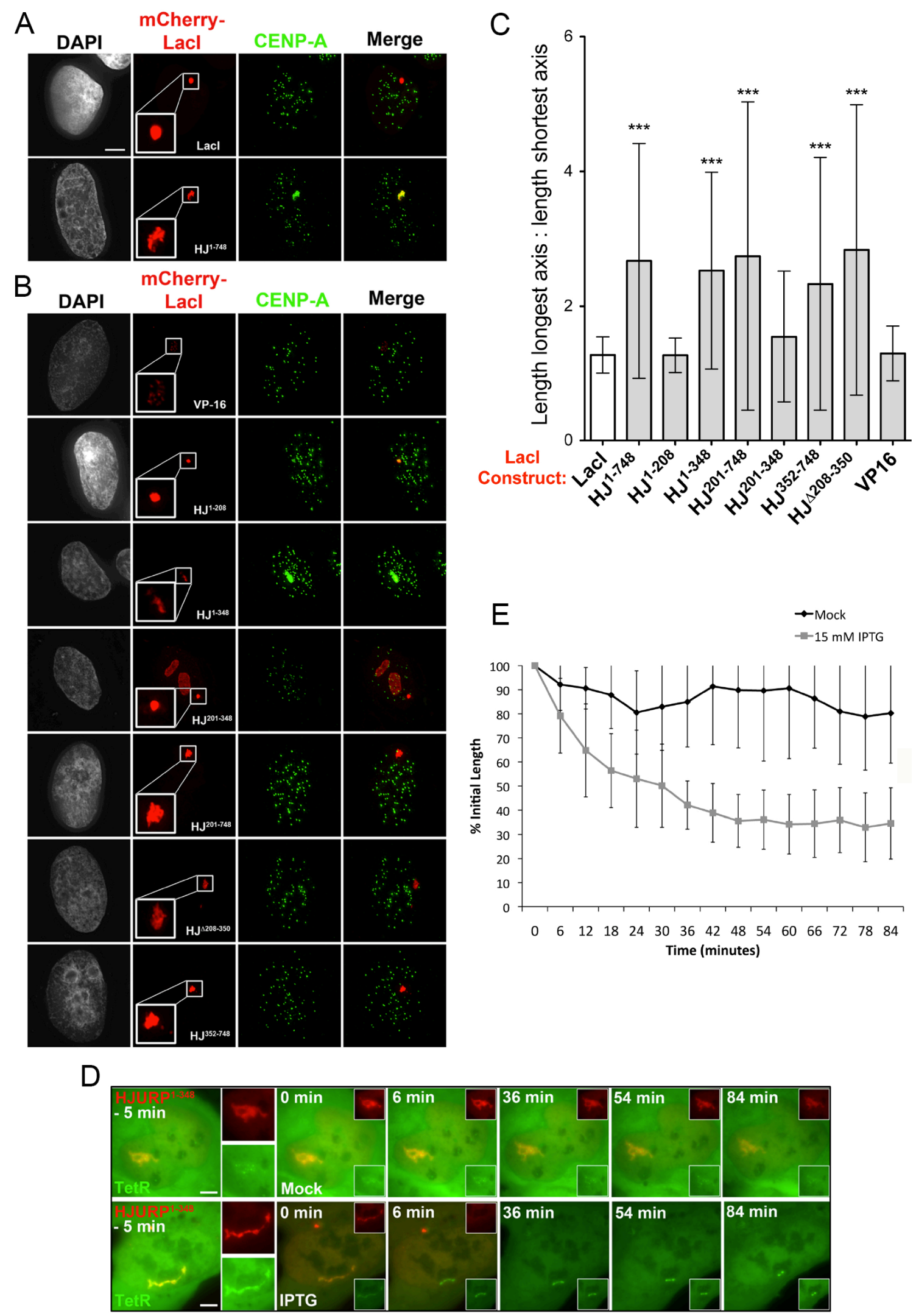


\section{HJURP interacts with the Condensin II complex}

The fact that CENP-A deposition occurs in early G1 just after the cell exits mitosis, instead of in S-phase like other canonical histones, presents the challenge of the chromatin being highly condensed at the time of deposition. An ability to decondense chromatin may therefore be valuable to HJURP's role as the CENP-A nucleosome assembly factor. It is plausible that additional proteins at the centromere contribute to regulating this decondensation. The condensin II complex is responsible for axial shortening of mitotic chromosomes and is known to work alongside the condensin I complex to efficiently condense mitotic chromosomes during G2 and prophase (Hirano, 2012). These proteins are localized all along chromosomes during mitosis, and the condensin II complex specifically enriches at centromeric chromatin during mitosis (Ono et al., 2004). Additionally, the condensin complexes have previously been implicated in affecting CENP-A deposition in both frogs and humans (Bernad et al., 2011; Samoshkin et al., 2009).

Because condensins are known regulators of chromatin condensation state with precedence in affecting CENP-A deposition, we decided to investigate if HJURP interacts with members of these complexes. U2OS-LacO cells were transfected with mCherry-LacI-HJ ${ }^{1-748}$ and either CAP-H-LAP (condensin I subunit) or CAP-H2-LAP (condensin II subunit). Cells were arrested in mitosis with nocodazole and prepared as metaphase spreads. mCherry-LacI-HJ ${ }^{1-748}$ was specifically able to recruit the condensin II subunit, CAP-H2-LAP, and not the condensin I subunit, CAP-H-LAP (Figure 4-2 A, B; Supp. Figure 4-2 A). In line with our analysis of interphase nuclei (Figure 4-1), in these mitotic spreads, the tip of chromosome 1 where the LacO/TRE array is integrated was 
visibly extended compared to the remainder of the mitotic chromosome when mCherryLacI-HJ ${ }^{1-748}$ was present (Figure 4-2 B; Supp. Figure 4-2 A). In order to quantify the specific recruitment of CAP-H2-LAP, its intensity was measured at the array and set as a ratio against the arm staining. CAP-H2 was significantly enriched at the $\mathrm{LacO}$ array compared to the condensin I CAP-H subunit (Figure 4-2 C). To verify the recruited condensin subunits could not cause a similar array lengthening through their axial compaction capability, we targeting mCherry-LacI-CAP-H or mCherry-LacI-CAP-H2 to the LacO array. Neither subunit induced array lengthening on its own indicating the decondensation induced by HJURP is not the result of CAP-H2 recruitment (Supp. Figure 4-2 B, C).

We next wanted to determine which region within HJURP is required to interact with the condensin II complex. Truncations of HJURP used in Figure 4-1 were targeted to the LacO array for 48 hours in cells co-transfected with CAP-H2-LAP. Similarly to the array decondensation, fragments of HJURP including amino acids 201-748 were also sufficient to recruit the condensin II subunit (Figure 4-2 D, E). The minimal region required to recruit CAP-H2-LAP was mCherry-LacI-HJ ${ }^{352-748}$, the same minimal region that induces array decondensation (Figure 4-1 B, C; Figure 4-2 D, E). We note that the interphase HJURP arrays do not extend as drastically when CAP-H2 is over-expressed and recruited, and the images displayed in Figure 4-2 D were selected to reflect this.

We additionally verified that condensin II recruitment was not a general response to chromatin condensation changes by targeting LacI-VP-16. While it changed the chromatin compaction at the array, LacI-VP-16 was not sufficient to recruit condensin II indicating condensin II recruitment by HJURP is specific and is not a general response to 
any type of chromatin condensation change (Appendix Figure 4-2 C, D). $\mathrm{HJ}^{1-748}$ was also able to efficiently immunoprecipitate CAP-H2-LAP from randomly cycling and mitotic cell extracts but could not immunoprecipitate CAP-H (Figure 4-2 F, Supp. Fig 4-2 D). This argues for a physical interaction between these two proteins, though does not completely rule out the possibility that condensin II is recognizing the structure induced by HJURP at chromatin. These data together suggest that HJURP and the condensin II complex interact and may work together to decondense then recondense centromeric chromatin during the process of CENP-A deposition. We therefore propose the HJURPcondensin II interaction is a regulatory one to control HJURP-induced chromatin decondensation in the process of CENP-A deposition. 
Figure 4-2: HJURP interacts with the Condensin II complex

(A) Representative images of mitotic spreads of U2OS-LacO cells transfected for 48 hours mCherry-LacI-HJ ${ }^{1-748}$ and CAP-H2-LAP or CAP-H-LAP. Centromeres are stained with CENP-A antibody. (B) Inset of boxed regions in A. (C) Quantification of experiment in A, represented as a ratio of GFP intensity at LacI-HJ ${ }^{1-748}$ positive arrays to GFP intensity on the adjacent chromosome arm. $\mathrm{n}=30$ arrays, $\mathrm{N}=2$ biological replicates. $\mathrm{p}<0.0002$ by t-test. (D) Representative images of U2OS-LacI cells co-transfected for 48 hours with indicated mCherry-LacI-HJ fragments and CAP-H2-LAP. Centromeres are stained with CENP-A antibody. Scale bar represents $5 \mu \mathrm{m}$. (E) Quantification of experiment in D. Graph displays intensity of CAP-H2-LAP at individual LacI-HJ arrays as a ratio over the average nuclear background. $n \geq 30$ arrays per condition, $N=1$ biological replicate. Asterisks indicate $\mathrm{p}<0.0001$ when compared to LacI by Kruskal-Wallis test followed by Dunn's Multiple Comparison test. Orange dotted line marks an intensity ratio of 1, indicating no array enrichment. (F) HA immunoprecipitation from HEK cells transfected for 24 hours with HA-HJ ${ }^{1-748}+/--$ CAP-H2-LAP. Mock IgG pull down and HA-HJ ${ }^{1-748}+$ CAP-H-LAP HA IP were used as negative controls. Npm1 is shown as a loading control. $\mathrm{N}=2$. 


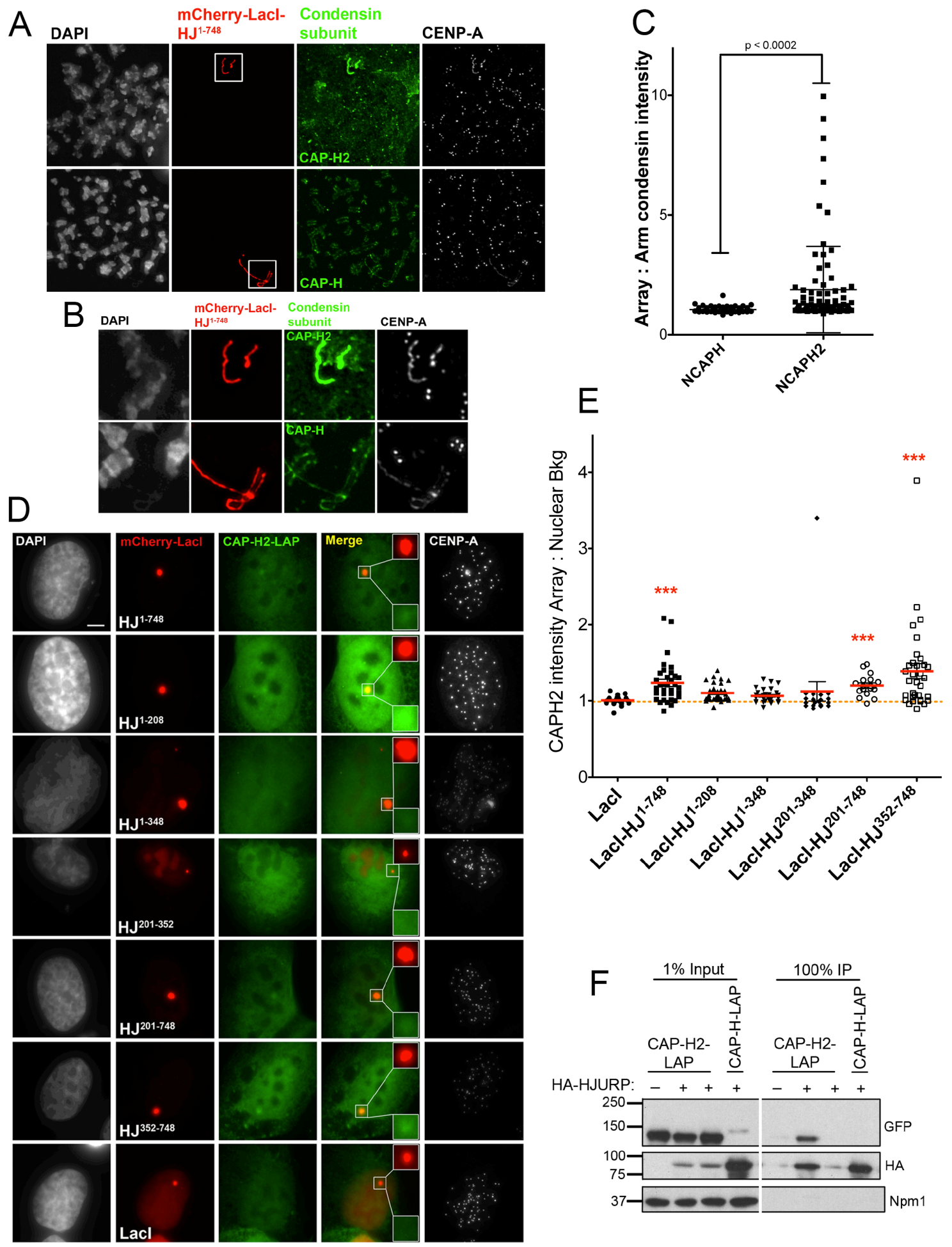




\section{Depleting the Condensin II complex enhances HJURP-dependent array decondensation}

If the role of condensin II complex recruitment is to counteract the chromatin decondensation induced by HJURP then depleting the condensin II complex should enhance the ability of HJURP to decondense chromatin at the array. To test this, U2OSLacO cells were transfected with mCherry-LacI-HJ ${ }^{1-748}$ followed by 48 hours of either negative control siRNA or siRNA to two condensin II complex subunits, CAP-H2 and CAP-D3 (Figure 4-3 B). Previous studies used siRNA to multiple subunits in order to effectively deplete the complex, so that was the approach we took as well (Ono et al., 2013).

Consistent with our hypothesis, we observed an increase in the length to width ratio of the LacI-HJ ${ }^{1-748}$ arrays with CAP-H2 + CAP-D3 siRNA treatment (Figure 4-3 A, C). The increase in array length to width in the population as a whole was modest but was statistically significantly different from treating with negative control siRNA (Figure 4-3 C). The modest effect may be due to the fact that the condensin complex is highly abundant in the cell and is difficult to deplete entirely, even with the use of multiple siRNA's (Figure 4-3 B). Despite this, several cells from different replicates displayed highly decondensed character following condensin II depletion, with lengths between 2030x their widths (Figure 4-3 A, C). This argues interaction between HJURP and the condensin II complex is necessary to moderate HJURP chromatin decondensation. We next investigated if this interaction between HJURP and the condensin complex has a role at the centromere by depleting the condensin II complex and assaying the effects this has on CENP-A deposition factor localization and on CENP-A loading. 
Figure 4-3: Depleting the Condensin II complex enhances HJURP-dependent array decondensation

(A) Representative images of U2OS-LacO cells transfected with mCherry-LacI-HJURP then treated with either negative control siRNA or CAP-H2 and CAP-D3 siRNA for 48 hours. Cells were stained with a CENP-A antibody. (B) Q-PCR analysis of CAP-H2 and CAP-D3 transcript levels relative to negative control siRNA following 48 hours siRNA treatment. GUS was used as an endogenous control transcript. Error bars represent standard deviation. $\mathrm{N}=2$ biological replicates. (C) Longest length versus widest width ratio measurements of experiments represented in A. Bar represents the mean, and whiskers mark the standard deviation. $\mathrm{p}=0.0206$ by $\mathrm{t}$-test. $\mathrm{n}=30$ arrays per condition, $\mathrm{N}=2$ biological replicates. 

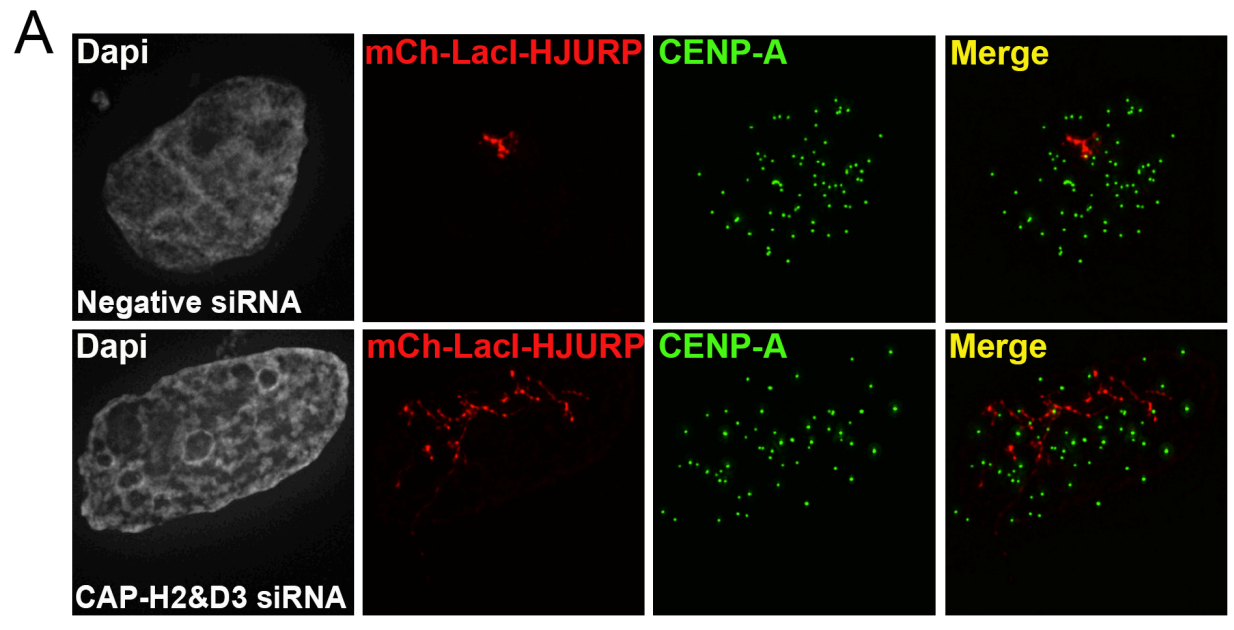

B
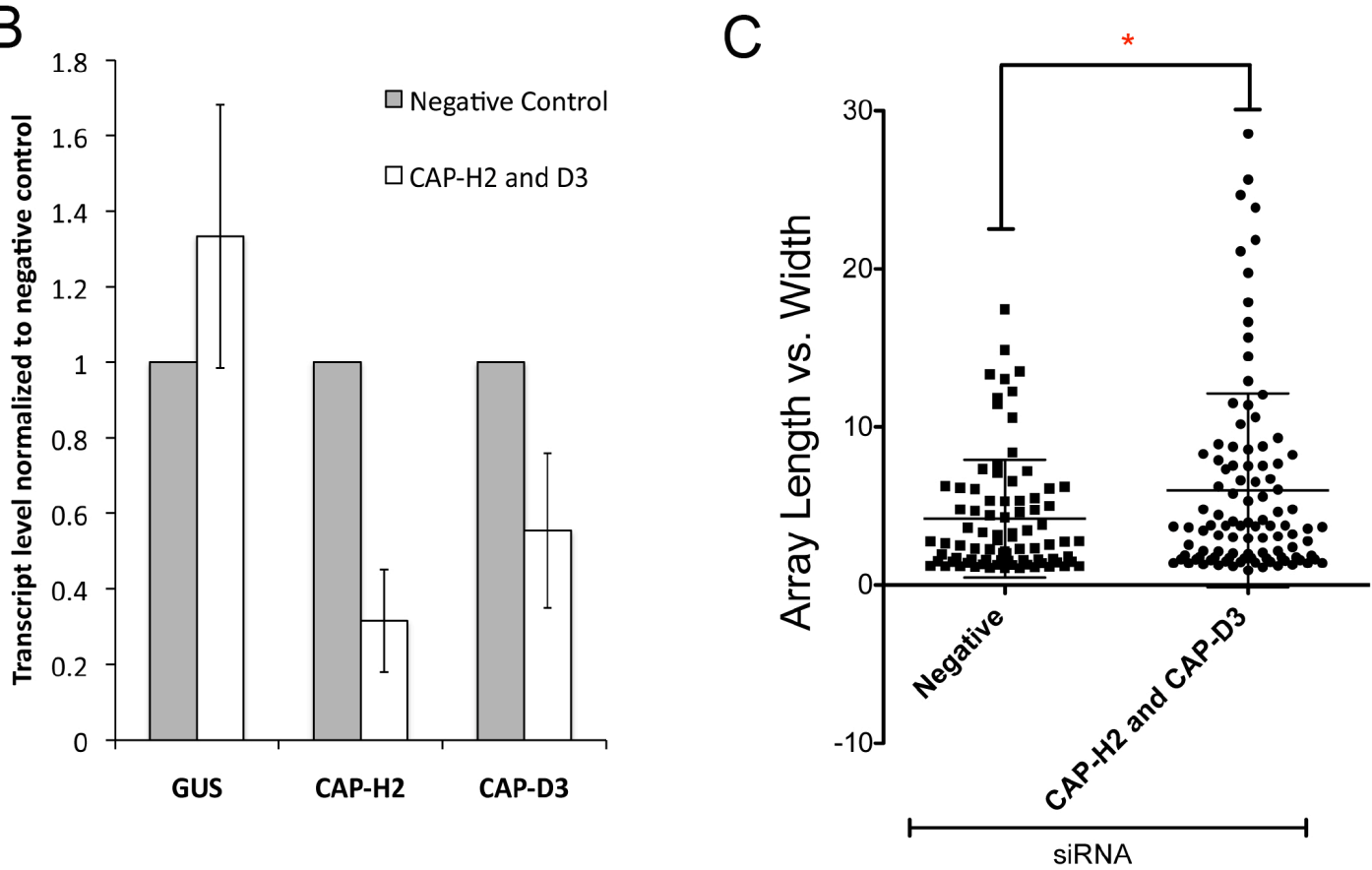


\section{Condensin II depletion reduces HJURP centromeric recruitment}

The Mis18 complex and HJURP both localize to centromeres in early G1 just as the cell exits mitosis. This localization initiates new CENP-A deposition so is a crucial step in maintaining centromeric chromatin integrity (Dunleavy et al., 2009; Foltz et al., 2009; Fujita et al., 2007). Because the condensin II complex is enriched at CENP-A chromatin during mitosis (Ono et al., 2004), just prior to when Mis18 and HJURP localize there, we wanted to investigate if the interaction between HJURP and the condensin II complex is important for HJURP's ability to localize to centromeres.

We depleted the condensin II complex (sub-units CAP-H2 and CAP-D3) from HeLa cells lines stably expressing Mis $18 \alpha-\mathrm{GFP}$ or $\mathrm{HJ}^{1-748}$-LAP. The percentage of cells with centromere-localized Mis18 $\alpha$-GFP or $\mathrm{HJ}^{1-748}$-LAP was then counted (Figure 4-4 A). Both subunits were depleted to approximately $40 \%$ of their endogenous levels (Figure 44 B). In negative control treated cells, Mis $18 \alpha$-GFP localized to centromeres in $38 \%$ of

cells and $\mathrm{HJ}^{1-748}$-LAP was at centromeres in $20 \%$ of cells (Figure $4-4 \mathrm{~A}, \mathrm{C}$ ). Upon condensin II depletion, the percentage of $\mathrm{HJ}^{1-748}$-LAP centromere-localized cells was statistically significantly reduced to $6.7 \%$. In contrast, the percentage of Mis $18 \alpha$-GFP centromere positive cells was unchanged (Figure 4-4 C). This reduction in HJURP centromere recruitment was not due to a mitotic arrest resulting in fewer cells entering into G1 because the mitotic index percentage was uniform between all conditions (Figure 4-4 D). These results indicate the interaction between HJURP and the condensin II complex is required for its proper centromeric recruitment or stability once it reaches the centromere. 
Figure 4-4: Condensin II depletion reduces HJURP centromeric recruitment

(A) Representative images of Mis18 $\alpha$-GFP HeLa or HJURP-LAP HeLa-TRex stable lines treated with negative control siRNA or CAP-H2 + CAP-D3 siRNA for 48 hours. Insets highlight centromere-localized examples for Mis18 $\alpha$-GFP and HJURP-LAP. Cells were stained with an antibody to CENP-A. (B) Q-PCR analysis of CAP-H2 and CAP-D3 transcript levels relative to negative control siRNA following 48 hours siRNA treatment. GUS was used as an endogenous control transcript. Error bars represent standard deviation. $\mathrm{N}=2$ biological replicates. (C) Quantification of experiment in A. Graph displays the percent out of $\geq 100$ cells of either Mis $18 \alpha$-GFP or HJURP-LAP centromerelocalized cells following negative control or CAP-H2 + CAP-D3 siRNA. Error bars represent standard deviation. Asterisks indicate a $\mathrm{p}=0.0017$ by t test. $\mathrm{N}=3$ biological replicates. (D) Graph displays percent mitotic cells from experiments represented in A. Error bars represent standard deviation. $\mathrm{p}=0.5079$ by Kruskal-Wallis test. $\mathrm{N}=3$ biological replicates. 


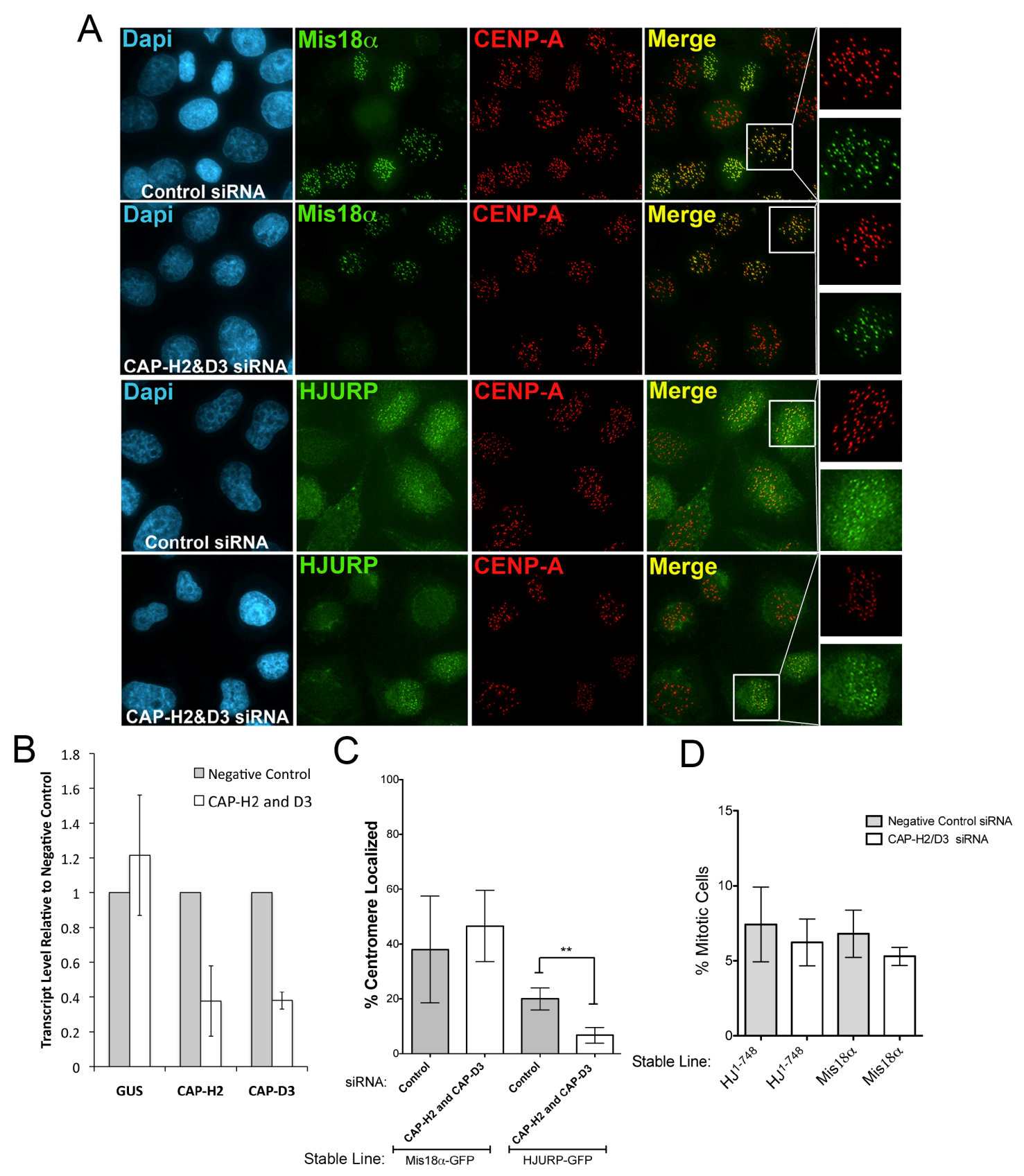




\section{Condensin II depletion results in CENP-A deposition defect in human cells}

Previous studies in Xenopus egg extracts demonstrated that depleting condensin II reduces new CENP-A loading at centromeres and that this could be partially rescued by exogenously adding back the complex (Bernad et al., 2011). Studies in human tissue culture cells have also implicated condensin involvement in the CENP-A deposition pathway (Samoshkin et al., 2009). However, the reduced CENP-A loading observed by Samoshkin et al. resulted from depleting both the SMC2 and SMC4 subunits, which are common to the condensin I and condensin II complexes. The fact that condensin II knockdown affects HJURP centromere localization (Figure 4-4) led us to investigate the role for specifically the condensin II complex in human CENP-A deposition.

HeLa-TRex cells expressing inducible mCherry-CENP-A were depleted of either HJURP or the condensin II specific complex members, CAP-H2 and CAP-D3. After 48 hours of siRNA treatment, mCherry-CENP-A expression was induced for 12 hours. This experimental set-up allowed us to specifically investigate the fate of the newly expressed mCherry-CENP-A under conditions where HJURP or the condensin II complex had already been depleted. Both the condensin II subunits and HJURP levels were significantly reduced compared to negative control treated cells following the siRNA treatment (Figure 4-5 C, D). As expected, HJURP depletion resulted in a complete lack of new mCherry-CENP-A deposition at centromeres (Figure 4-6 A, B) (Dunleavy et al., 2009; Foltz et al., 2009). Interestingly, depleting the condensin II complex also resulted in decreased mCherry-CENP-A levels at centromeres. The average mCherry-CENP-A centromeric intensity was statistically significantly reduced to approximately $50 \%$ of the negative control treated intensity (Figure 4-5 A, B). We conclude that HJURP interaction 
with the condensin II complex is necessary for its centromeric recruitment or stability, and as such, loss of interaction between condensin II and HJURP results in reduced CENP-A loading at human centromeres. The reduction in newly loaded CENP-A at centromeres upon condensin II depletion may additionally be attributed to active participation of the condensin II complex in the CENP-A deposition process, perhaps by recondensing centromeric chromatin following HJURP-induced decondensation for deposition. 
Figure 4-5: Condensin II depletion results in CENP-A deposition defect in human cells

(A) Representative images of HeLa-TRex cells expressing inducible mCherry-CENP-A. Cells were treated for 36 hours with negative control, HJURP, or CAP-H2 + CAP-D3 siRNA, then mCherry-CENP-A expression was induced for 12 hours. Cells were immunostained with CENP-T antibody. Scale bar is $5 \mu \mathrm{m}$. (B) Quantification of centromeric mCherry-CENP-A intensity from experiment represented in A. $\mathrm{n} \geq 90$ centromeres from multiple microscope fields, $\mathrm{N}=2$ biological replicates. Bar represents the mean and whiskers mark the standard deviation. Asterisks indicate $p<0.0001$ as compared to negative control by Kruskal-Wallis test. (C) Q-PCR analysis of CAP-H2 and CAP-D3 transcript levels relative to negative control siRNA following 48 hours siRNA treatment. GUS was used as an endogenous control transcript. Error bars represent standard deviation. $\mathrm{N}=2$ biological replicates. (D) Immunoblot of cell lysates from experiment represented in A demonstrating HJURP depletion efficiency. Tubulin was used as a loading control. 


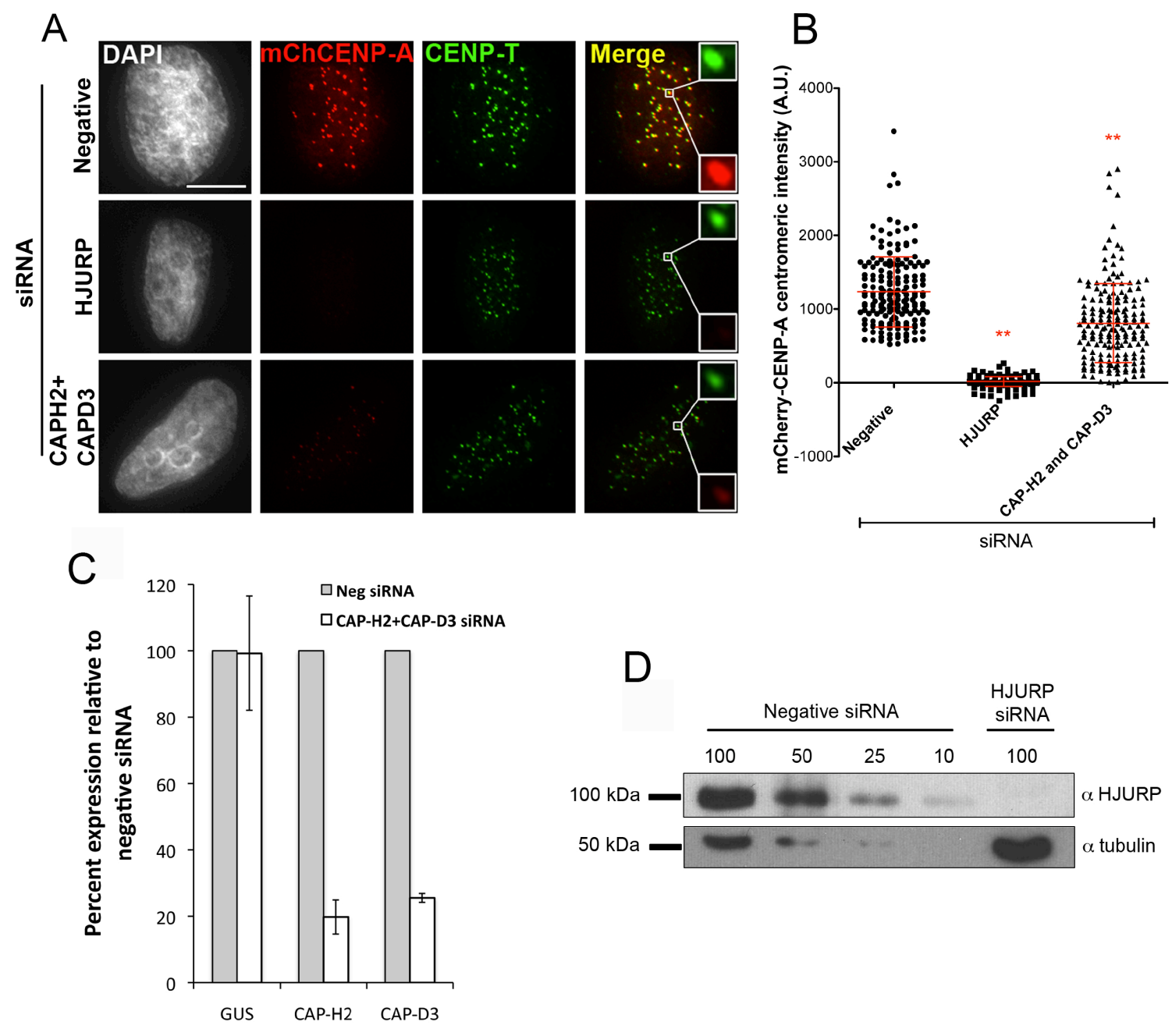


Figure 4-6: Model of condensin II and HJURP at the centromere during CENP-A deposition

HJURP is recruited to centromeric chromatin in early G1 by the Mis 18 complex. HJURP brings new CENP-A with it to the centromere. Once at centromeric chromatin, HJURP decondenses the chromatin there and loads new CENP-A into the chromatin. Condensin II is locally enriched with HJURP and recondenses the chromatin after HJURP deposits CENP-A leading to stable new CENP-A assembly. Condensin II is also involved in stably recruiting or keeping HJURP at the centromere in early G1, so they are drawn as interacting at that time. 


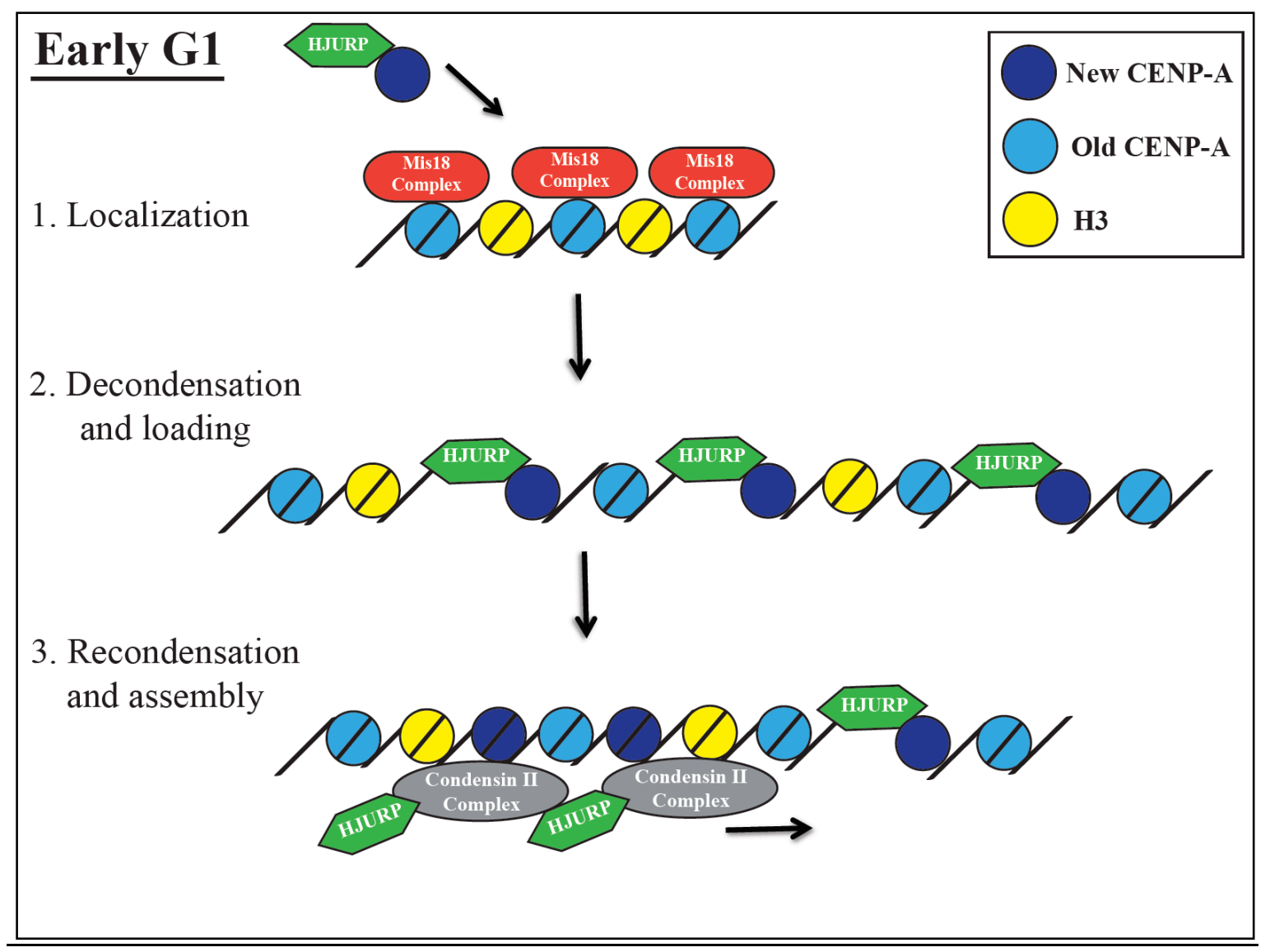




\section{$\underline{\text { Discussion }}$}

Centromeric chromatin establishment and maintenance are cell cycle driven processes that are essential for chromosome stability and inheritance during mitosis. The CENP-A chaperone HJURP and its recruitment machinery, the Mis18 complex, are currently considered two of the major players in CENP-A deposition in human cells. Here we show HJURP can induce chromatin decondensation in vivo when it is targeted to chromatin (Figure 4-1). The C-terminal portion of HJURP from amino acids 352-748 is sufficient to induce this decondensation (Figure 4-1). Consistent with the array expansion induced by HJURP being chromatin decondensation, condensin complex subunits are recruited to the array when portions of HJURP inducing the expansion are targeted. We additionally found that HJURP can interact specifically with the condensin II complex (Figure 4-2). In support of this interaction being necessary for centromeric chromatin establishment, condensin II subunit depletion reduces HJURP's centromeric localization (Figure 4-4). Finally, we show that CENP-A deposition is also reduced by $50 \%$ when the condensin II complex is depleted (Figure 4-5). These data support a model where the chromatin decondensation activity of HJURP requires modulation by the condensin II complex. Condensin II is present at the centromeres during mitosis, and we hypothesize becomes locally enriched by interaction with HJURP during CENP-A loading. This interaction is required for CENP-A deposition in human cells (Figure 4-6).

The fact that CENP-A deposition occurs in early G1 just after the cell exits mitosis instead of in S-phase like other canonical histones presents a challenge of the chromatin being highly condensed at the time of deposition. As such, it is critical for the initial CENP-A deposition step in early G1 to be compatible with highly condensed 
chromatin. The CENP-A nucleosome assembly factor, HJURP, must be able to assemble new CENP-A nucleosomes onto this highly condensed chromatin. We demonstrate here that HJURP or a closely bound interacting protein induces chromatin decondensation when targeted to chromatin (Figure 4-1). We propose this decondensation observed by tethering HJURP to chromatin may also be involved when HJURP deposits CENP-A onto a condensed chromatin template in early G1. In order to prevent aberrant chromatin decondensation, this activity of HJURP must be regulated in the cell. Our data indicate that recruitment of the condensin II complex may act to regulate where HJURP can decondense chromatin and to assist in re-condensing the chromatin after CENP-A has been deposited (Figure 4-6).

HJURP's ability to decondense chromatin would need to be highly regulated in the cell to prevent aberrant deposition. Localization regulation would be one way to dictate when and where the decondensation would occur. Consistent with this, HJURP only localizes to centromeric chromatin during a short window in early G1, and this recruitment requires upstream localization of additional proteins, the Mis18 complex (Barnhart et al., 2011; Dunleavy et al., 2009; Foltz et al., 2009). I have included preliminary data as an appendix to this chapter that implicates Mis $18 \alpha$ as another potential regulator of HJURP decondensation. This will be discussed in more detail in that section. Throughout the rest of the cell cycle, HJURP is localized away from the centromere primarily in the nucleolus where is may have another life separate from CENP-A deposition. HJURP was previously implicated in the homologous recombination pathway for double strand break repair and was shown to bind to synthetic Holliday junctions in vitro (Kato et al., 2007). Nothing is known about HJURP's 
mechanistic role in DNA damage, and it is possible that the decondensation phenomenon we have characterized here and HJURP's interaction with condensin II may also be required in HJURP's role in DNA damage.

The chromatin decondensation induced by HJURP at the LacO arrays is highly dynamic. Movies taken of the expanded arrays revealed that they expand, contract, and move on a second by second timescale, and the expansion of the array is reversible (Data not shown; Figure 4-1). This implies that this decondensation is an active process that may require energy through the hydrolysis of ATP. HJURP purifications from HeLa cells found that it co-purifies with Tip49a/Tip48b, which are RuvB-like AAA ATPases (Shuaib et al., 2010). Their co-purification with HJURP suggests it has access to ATPases to fuel chromatin decondensation. Future investigations will need to determine if Tip49a/Tip48b are required for HJURP chromatin decondensation.

HJURP is sufficient to deposit CENP-A nucleosomes both in vitro and in vivo, and this has been demonstrated in both flies and humans (Barnhart et al., 2011; Chen et al., 2014). In vitro nucleosome assembly assays suggest that HJURP is sufficient and specific for depositing CENP-A. However, in these studies, the efficiency of HJURP nucleosome assembly was consistently less than using a general histone chaperone like dNap1. Additionally, tethering HJURP to LacO arrays resulted in stable CENP-A deposition, but only a portion of the recruited CENP-A was efficiently deposited (Barnhart et al., 2011). This implies that other factors must be present at endogenous centromeres to assist HJURP in the deposition process. We show here HJURP interacts with the condensin II complex. HJURP has previously been shown to co-purify with Npm1, RbAP46/48, and Tip49a/Tip49b - all known members of chromatin remodeling 
complexes (Shuaib et al., 2010). All these proteins may contribute to CENP-A loading in some aspect, and future studies will be needed to understand the mechanism by which each contributes.

Lastly, our data support a model where the condensin II complex would work alongside HJURP to re-condense the chromatin after the new CENP-A is deposited. We show that depleting the condensin II complex resulted in magnified chromatin decondensation at the LacO array (Figure 4-3). In addition, specifically condensin II depletion induced decreased new CENP-A loading at the centromere (Figure 4-5). This is consistent with previous results in yeast, frogs, and humans where depleting condensin subunits also reduced CENP-A/Cse4 loading (Bernad et al., 2011; Samoshkin et al., 2009; Yong-Gonzalez et al., 2007). In conclusion, the fact that CENP-A deposition occurs in early G1 instead of in S-phase presents the challenge of the chromatin being highly condensed at the time of deposition. As such, it is critical for HJURP to be able to assemble new CENP-A nucleosomes onto highly condensed chromatin. We propose here that HJURP possesses inherent chromatin decondensation activity that could enable deposition onto a condensed span of chromatin. Regulating this process would be crucial, and we describe a model where the temporal and spatial control of HJURP centromeric recruitment by the Mis 18 complex is combined with the condensation activity of the condensin II complex to allow for controlled HJURP-dependent chromatin decondensation required for successful CENP-A deposition (Figure 4-6). A future directions section is included after the appendix to discuss the experiments that are in progress to mechanistically understand what HJURP is doing to the chromatin. 


\section{$\underline{\text { Supplemental Figures }}$}

Supplemental Figure 4-1: Array decondensation does not reduce H3 occupancy and Npm1 does not alter array condensation state

(A) $\mathrm{H} 3$ signal at $\mathrm{LacO}$ array relative to negative control locus from ChIP experiments from U2OS-LacO cells transfected for 48 hours with mCherry-LacI, mCherry-LacI-HJ ${ }^{1-}$ ${ }^{208}$, mCherry-LacI-HJ ${ }^{201-748}$, or mCherry-LacI-HJ ${ }^{1-748}$. DNA was purified from IP's using antibodies to $\mathrm{H} 3$ or $\mathrm{IgG}$ then probed for $\mathrm{LacO}$ sequences. Error bars represent standard deviation. $\mathrm{N}=3$ biological replicates. (B) Live cell images of U2OS-LacO cells transfected for 24 hours with LacI-Npm1 and YFP-CENP-A. Error bar represents $5 \mu \mathrm{m}$. (C) Quantification of experiment in B. \% of LacI-Npm1 positive arrays that are round, misshapen, or extended. $\mathrm{n}=30$ arrays counted. $\mathrm{N}=1$ biological replicate. 

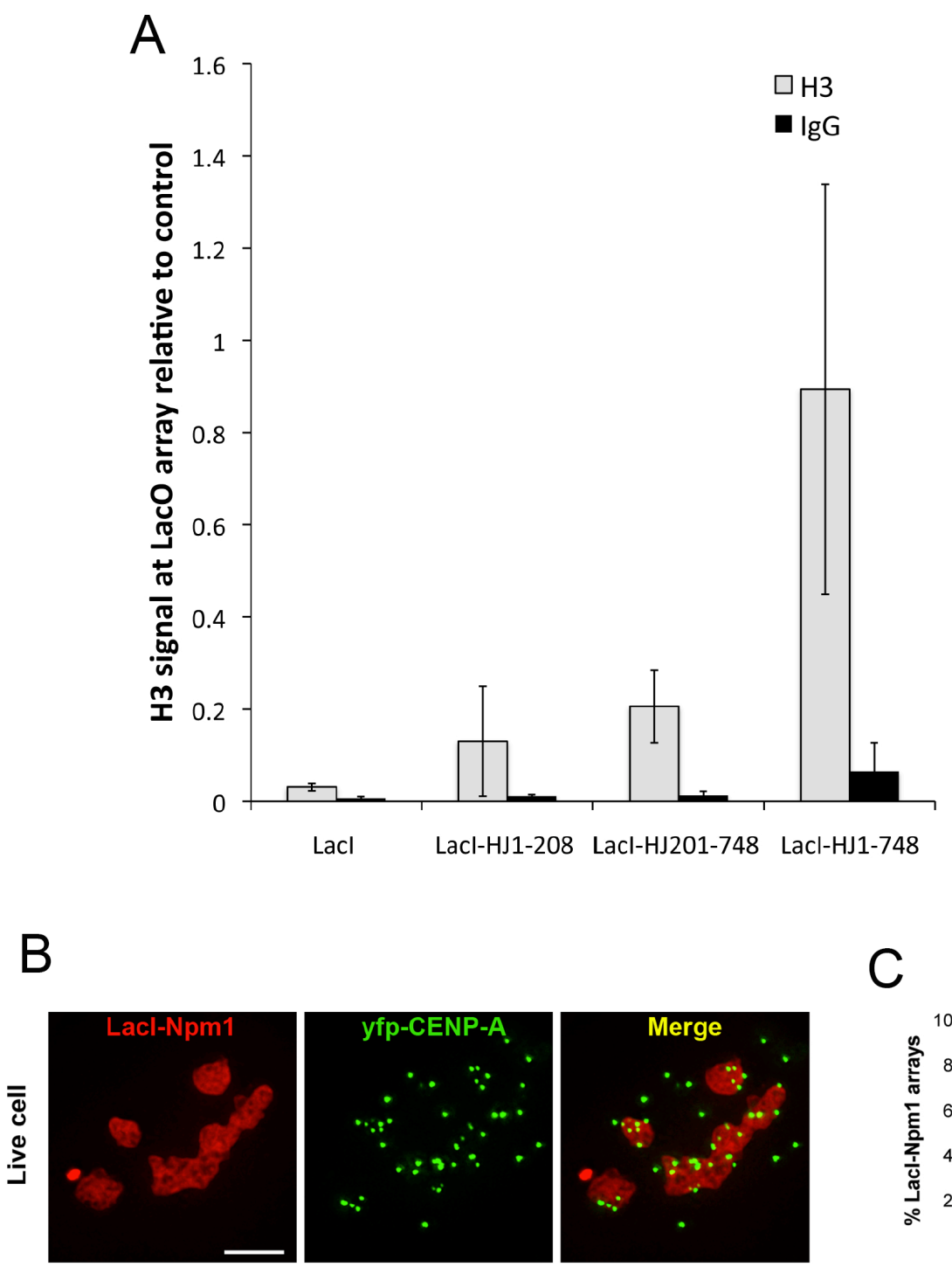

C

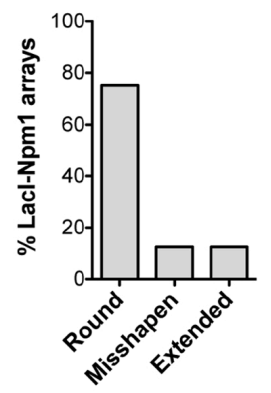


Supplemental Figure 4-2: Condensin subunits do not induce array expansion and CAPH2 can interact with HJURP during mitosis and in randomly cycling cells

(A) Additional mitotic spread examples of U2OS-LacO cells transfected for 48 hours with mCherry-LacI-HJURP and CAP-H or CAP-H2-LAP. CENP-A is stained as a centromere marker. (B) Representative images of U2OS-LacO cells transfected for 48 hours with mCherry-LacI-CAP-H or mCherry-LacI-CAP-H2. Cells were stained with a CENP-A antibody to mark centromeres. (C) Quantification of experiment in A displayed as the ratios of the longest axis to the shortest axis for $n=20$ arrays. (D) HAimmunoprecipitation from HEK cells transfected for 48 hours with HA-HJ and CAP-H2LAP, +/-- nocodazole treatment to block cells in mitosis. Blots were probed for HA, GFP, and Npm1 as a loading control. 


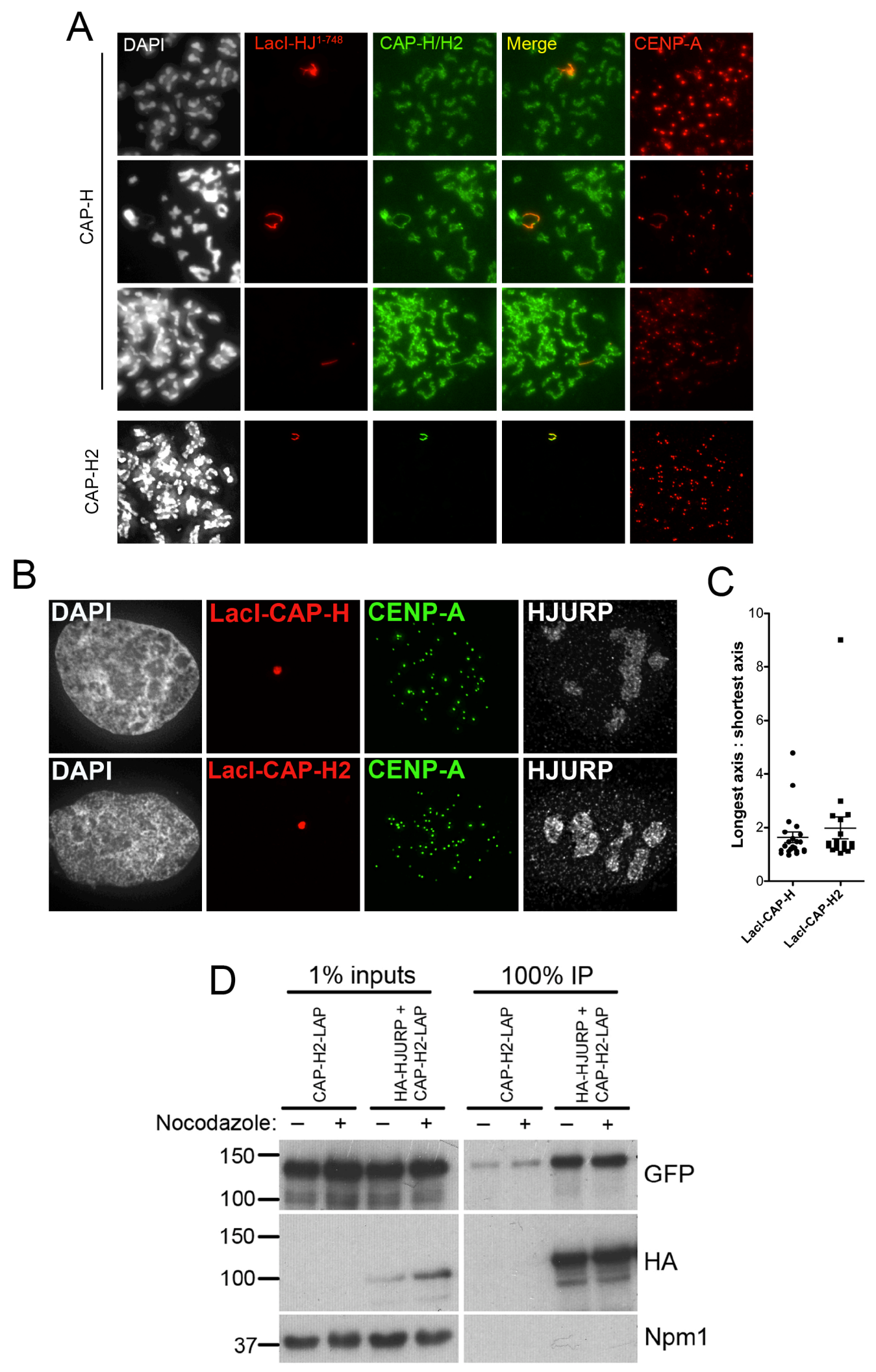




\section{$\underline{\text { Data Appendix }}$}

We have done preliminary investigations looking at the role the Mis18 complex may play on HJURP's ability to decondense chromatin. The data are set aside from the main figure set because they are not fully solidified but have yielded some interesting results.

Mis 18a co-targeting can inhibit HJURP chromatin decondensation

To determine if the chromatin decondensation property of HJURP is important for proper CENP-A deposition, we investigated if other proteins in the CENP-A deposition pathway could influence the array condensation. The human Mis18 complex, composed of Mis18 $\alpha$, Mis18 $\beta$, and Mis18BP1, recruits HJURP to centromeres and is required for CENP-A deposition (Barnhart et al., 2011; Fujita et al., 2007). Mis18 $\alpha$ and Mis18 $\beta$ have previously been shown to affect histone acetylation and DNA methylation status at centromeric chromatin (Fujita et al., 2007; Kim et al., 2012). Therefore we decided to investigate if they could influence the array decondensation.

Unlike HJURP, targeting mCherry-LacI-Mis18 $\alpha$ to the LacO/TRE array did not change the chromatin compaction at the array. However, when Mis18 $\alpha$ was co-targeted with mCherry-LacI-HJ ${ }^{1-748}$, it prevented HJURP from decondensing the array (Appendix Figure 4-1 A, B). This was not due to less mCherry-LacI-HJ ${ }^{1-748}$ being present as staining with an HJURP antibody indicated its intensity at the array did not change when mCherry-LacI-Mis18 $\alpha$ was co-targeted (Appendix Figure 4-1 C). In contrast, Mis18 $\beta$ was not able to prevent HJURP chromatin decondensation (Appendix Figure 4-1 A, B). Consistent with Mis18 $\alpha$ and HJURP harboring the ability to alter DNA compaction state, 
both proteins bound to DNA in an in vitro pull-down assay, whereas Mis18 $\beta$ could not bind (Appendix Figure 4-1 D).

Mis $18 \alpha$ contains a predicted C-terminal coiled coil that is required for its interaction with Mis18ß in vivo (Nardi, et al. Unpublished results 2014). Additionally, both Mis18 $\alpha$ and Mis18 $\beta$ contain putative metal binding Yipee CxxC motifs (YM) that are required for their centromere localization (Fujita et al., 2007). We next investigated whether mutating either of these regions within Mis18 $\alpha$ could disable it from recondensing the array. Mis $18 \alpha$ missing its coiled coil region could still induce compaction upon co-targeting with mCherry-LacI-HJ ${ }^{1-748}$. The coiled coils of Mis $18 \alpha$ and Mis18 $\beta$ are required for them to interact with each other as a heterotetramer in the cell. The coiled coils on their own do not target to centromeres and are solely important for bringing the Mis18 $\alpha$-Mis18 $\beta$ complex together (Nardi, et al. Unpublished results 2014). In contrast, co-targeting the centromere localization mutant mCherry-LacI-Mis $18 \alpha^{\mathrm{CxxC}}$ with mCherry-LacI-HJ ${ }^{1-748}$ resulted in arrays that were still able to decondense (Appendix Figure 4-1 E, F). The CxxC motifs within Mis18 $\alpha$ may therefore be required for its DNA binding activity, which may contribute to condensation in some way. Alternatively, the CxxC region of Mis18 $\alpha$ is also required for it to interact with Mis18BP1 so may be required for condensation because it is recruiting Mis18BP1 to the array (Stellfox et al. Under review 2014). It is therefore necessary to investigate if Mis18BP1 has any effect on the chromatin compaction at the array. 
Appendix Figure 4-1: Mis18a co-targeting can inhibit HJURP-induced decondensation.

(A) Representative images of U2OS-LacO cells transfected for 48 hours with mCherryLacI-Mis18 $\alpha$, mCherry-LacI-HJ ${ }^{1-748}$, mCherry-LacI-Mis $18 \beta$ alone, or mCherry-LacI-HJ ${ }^{1-}$ 748 co-transfected with either mCherry-LacI-Mis18 $\alpha$ or mCherry-LacI-Mis18 Centromeres are stained with an antibody to CENP-A. Scale bar represents $5 \mu \mathrm{m}$. (B) Quantification of experiments represented in A. HJURP antibody positive arrays in each condition were classified as extended, misshapen, or round. Schematic in legend provides representation of each of these phenotypes. $\mathrm{n} \geq 30$ arrays per condition, $\mathrm{N}=2$ biological replicates. Error bars represent standard deviation. (C) Quantification of mCherry, HJURP, and CENP-A intensity at the LacO array in cells transfected with mCherry-LacI$\mathrm{HJ}^{1-748}$ alone or co-transfected with mCherry-LacI-HJ ${ }^{1-748}+$ mCherry-LacI-Mis18 $\alpha . \mathrm{n}=6$

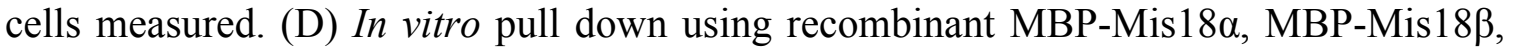
MBP-HJ ${ }^{1-348}$, or MBP alone that were incubated +/-- biotinylated $2 \mathrm{x} \alpha$-satellite DNA then pulled down using streptavidin magnetic beads. Immunoblotted for MBP. (E) Representative images of U2OS-LacO cells transfected for 48 hours with mCherry-LacIMis $18 \alpha^{\Delta \mathrm{CC}}$ (C-terminal coiled coil deletion), mCherry-LacI-Mis $18 \alpha^{\mathrm{CxxC}}$ (YM motif mutant), or mCherry-LacI-HJ ${ }^{1-748}$ co-transfected with each of these. Immunostained as in A. (F) Quantification of experiments represented in E. Arrays in each image were classified as extended, misshapen, or round. $\mathrm{n} \geq 30$ arrays per condition, $\mathrm{N}=1$ biological replicate. 


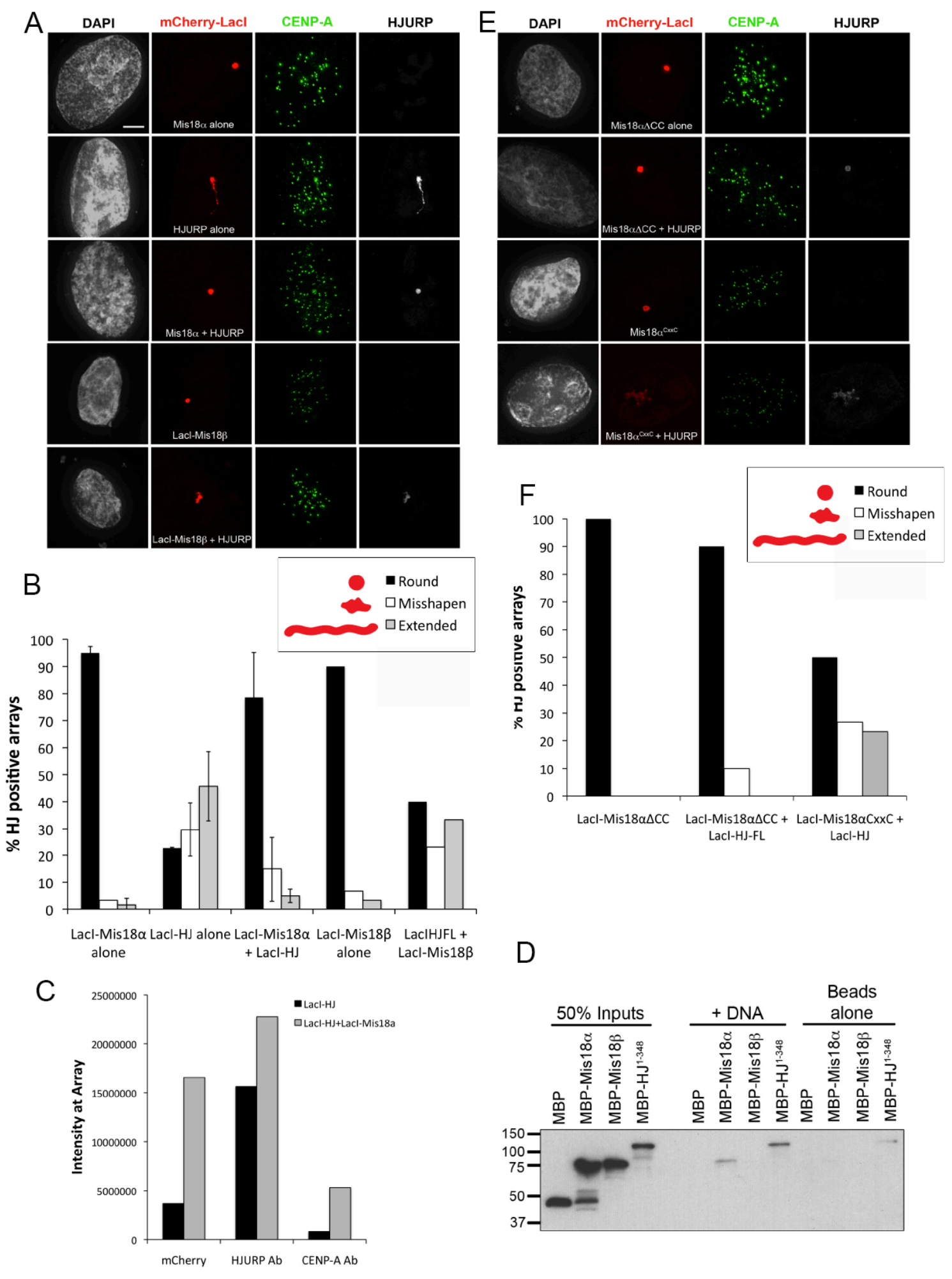


Mis 18a co-targeting can bypass the requirement for condensin II recruitment

Because Mis $18 \alpha$ was inhibitory to $\mathrm{HJ}^{1-748}$ chromatin decondensation, we tested whether co-tethering Mis18 $\alpha$ could also prevent condensin II recruitment (Appendix Figure 4-2 A-D). Co-tethering mCherry-Mis $18 \alpha$ to the LacO array with mCherry-LacI$\mathrm{HJ}^{1-748}$ reduced both the number of arrays recruiting CAP-H2-LAP and the intensity of any CAP-H2-LAP still present at the array (Appendix Figure 4-2 B, D). We observed a ring-like exclusion of the CAP-H2-LAP when Mis18 $\alpha$ was co-targeted to the array with LacI-HJ ${ }^{1-748}$ (Appendix Figure 4-2 A, C). This suggests that Mis18 $\alpha$ is sufficient to control the chromatin decondensation induced by HJURP, and this may bypass the requirement for recruiting condensin II to the $\mathrm{LacO}$ array. Whether Mis $18 \alpha$ replaces the need for condensin II enrichment to inhibit HJURP decondensation in the context of the centromere is not known. 
Appendix Figure 4-2: Mis18 1 inhibits Condensin II recruitment to LacI-HJ arrays

(A) Live cell representative images of U2OS-LacO cells transfected for 24 hours with mCherry-LacI-HJ ${ }^{1-748}$, mCherry-LacI-HJ ${ }^{1-208}$, mCherry-LacI-Mis18 $\alpha$, or mCherry-LacI$\mathrm{HJ}^{1-748}+$ mCherry-LacI-Mis18 $\alpha$. Insets highlight ring pattern of CAP-H2-LAP when it encounters mCherry-LacI-Mis18 $\alpha$. (B) Quantification of experiment in A - \% arrays with CAP-H2-LAP present. $\mathrm{n}=30$ arrays per condition. (C) Representative images of U2OSLacO cells transfected for 48 hours with mCherry-LacI-HJ fragments, mCherry-LacIMis $18 \alpha$, or mCherry-LacI-HJ + mCherry-LacI-Mis18 $\alpha$. mCherry-LacI-VP16 was used as a negative control for CAP-H2-LAP recruitment. (D) Quantification of experiment in C. Graph exhibits CAP-H2 intensity at the array minus average nuclear background. $\mathrm{n}=30$ arrays per condition. $\mathrm{N}=1$ biological replicate. 


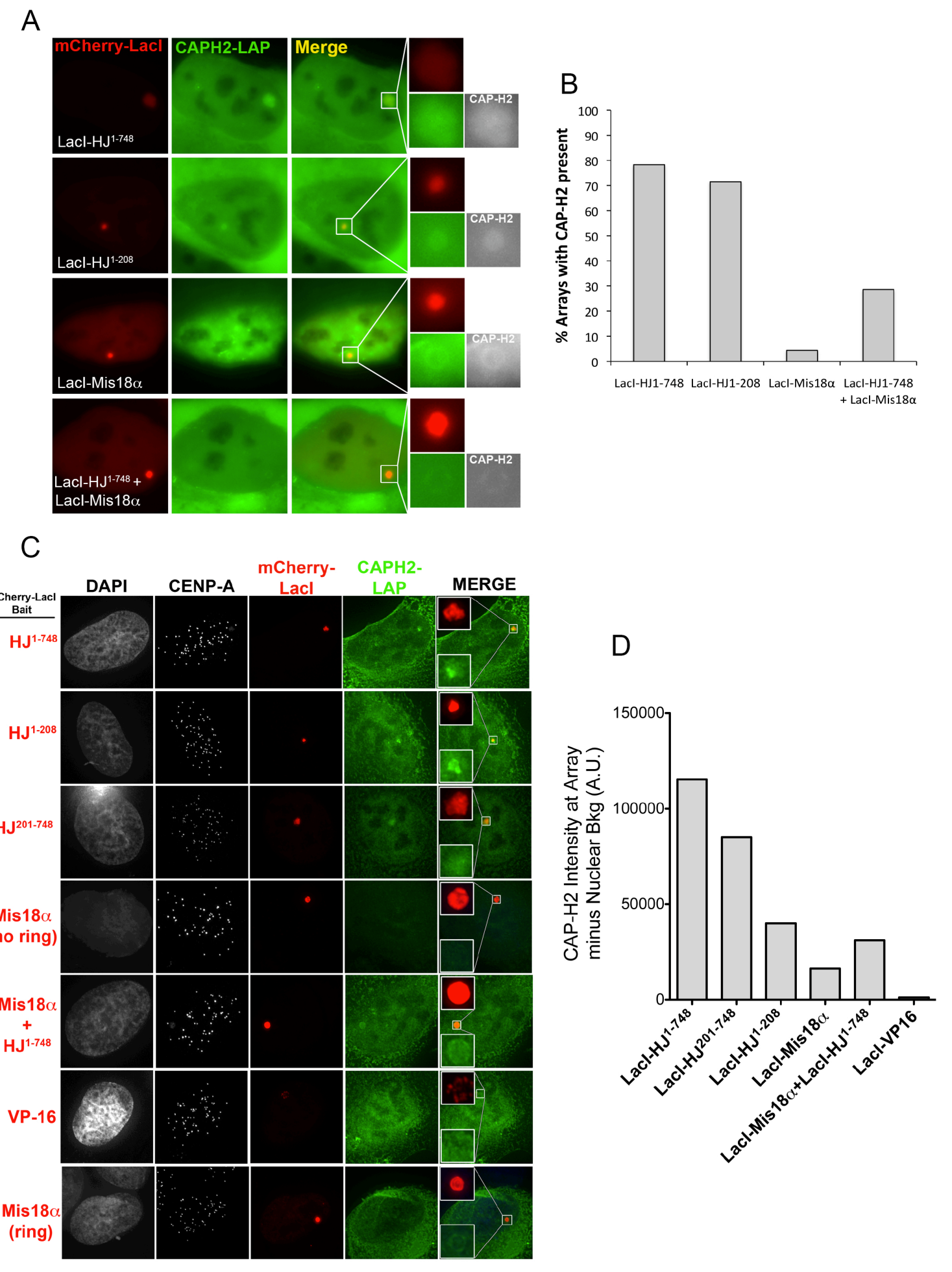




\section{$\underline{\text { Appendix Discussion }}$}

In order to prevent aberrant chromatin decondensation, this activity of HJURP must be regulated in the cell. Our data indicate that the Mis18 complex and recruitment of the condensin II complex may act in concert to regulate when HJURP can decondense chromatin and then to assist in re-condensing the chromatin after CENP-A has been deposited. One way the Mis18 complex could regulate HJURP's chromatin decondensation activity is by dictating when and where HJURP associates with chromatin. HJURP only localizes to centromeric chromatin during a short window in early G1, and this recruitment requires the Mis18 complex (Barnhart et al., 2011; Dunleavy et al., 2009; Foltz et al., 2009). The exact mechanism for how Mis18 recruits HJURP to centromeric chromatin is still under investigation. Recent studies from our lab identified a direct interaction between HJURP and Mis18 $\alpha$ and Mis18 $\beta$ (Nardi et al. Unpublished Results 2014). Previously, interactions between the Mis18 proteins and either CENP-A/Cnp1 or HJURP/Scm3 have not been robustly detected in vivo, and given that CENP-A deposition is contained to a short window in early G1, these data together are consistent with a physical HJURP-Mis18 interaction being highly regulated and short-lived in the cell. This also fits with a model where HJURP-induced chromatin decondensation at the centromere would be limited to a small window in time when CENP-A is actively being deposited.

Along with a direct interaction with HJURP, the Mis18 complex may epigenetically prime centromeric chromatin to make it compliant for HJURP recruitment and CENP-A deposition. The presence of the Mis18 complex influences histone 
acetylation at centromeric repeats and DNA methylation at centromeric chromatin (Fujita et al., 2007; Gopalakrishnan et al., 2009; Hayashi et al., 2004; Kim et al., 2012). Mouse embryonic fibroblasts lacking Mis $18 \alpha$ have a chromatin condensation defect in prometaphase providing in vivo evidence that Mis $18 \alpha$ may be able to condense chromatin in some way (Kim et al., 2012). The interaction of the Mis18 proteins with Mis16/RbAP46/48 also suggests they may play a role in chromatin remodeling at the centromere (Fujita et al., 2007; Hayashi et al., 2004). Our data demonstrating Mis18a's ability to inhibit HJURP's chromatin decondensation activity, and our preliminary studies that Mis $18 \alpha$ can bind to $\alpha$-satellite DNA suggest there is more to investigate about the interaction between Mis18 $\alpha$ and DNA.

The exact amount of time HJURP and the Mis18 proteins co-localize at the centromere is not known. Recent work from our lab proposes that HJURP binding to the Mis18 $\alpha$-Mis18 $\beta$ heterotetramer at the centromere causes this heterotetramer to be broken into two heterodimers and results in the Mis18 complex leaving the centromere (Nardi et al, Unpublished Results 2014). It is therefore plausible that Mis18 is crucial to recruit HJURP and prepare the proper chromatin environment for CENP-A deposition but then must be removed so HJURP can decondense centromeric chromatin in order to properly deposit CENP-A.

In total, these data suggest that condensin II and Mis18 $\alpha$ have a similar effect in countering HJURP-induced decondensation. It will need to be determined if Mis $18 \alpha$ functionally replaces the condensin II complex in this role at the centromere or if both are involved. The condensin II subunit CAP-H2 recruits to $\mathrm{LacI}-\mathrm{HJ}^{1-748}$ arrays more robustly 
than Mis $18 \alpha$, and this is one argument for the condensin II complex playing a primary role in the HJURP condensation process. 


\section{$\underline{\text { Future Directions }}$}

Demonstrate HJURP chromatin decondensation in vitro and determine the responsible region

We have conclusively shown that HJURP can induce a chromatin change at the LacO/TRE arrays. Our data support this change being HJURP-dependent because the array constricts immediately when HJURP is removed (Figure 4-1). A missing experiment in this work so far is to show whether HJURP can directly decondense chromatin in vitro. One way to approach this is using FRET between two positioned CENP-A nucleosomes. Differentially labeling each nucleosome with two fluorophores would allow them to FRET if in close enough contact. If we then add recombinant HJURP, we would predict that it would decrease FRET by decondensing the DNA template and separating the two fluorophores from each other. Alternatively, this can be done using an in vitro supercoiling assay where decondensation by HJURP should decrease the mobility of a plasmid DNA, though this may be difficult to interpret since HJURP binds to DNA (Kato et al., 2007). Additionally, it would be interesting to know if the decondensation is ATP-dependent. This can be done by presenting the cell with nonhydrolyzable ATP analogs and asking if decondensation is inhibited.

Another important aspect to investigate is the role of the HJURP-associated ATPases, Tip49a/Tip49b, in this process (Shuaib et al., 2010). HJURP does not contain any predicted ATPase domains, and because the observed chromatin decondensation is dynamic, it may also require ATP hydrolysis, though this needs to be tested. Therefore, these proteins may be directly involved in the chromatin decondensation process. Lastly, 
identifying point mutations within HJURP to narrow down which region of the protein is required would allow us to determine if centromeric chromatin decondensation is required for CENP-A deposition. The minimal region that decondenses the array, $\mathrm{HJ}^{352-}$ 748 (Figure 4-1 B, C), contains the centromere targeting domain of HJURP so cannot be deleted, and targeting it alone lacks the Scm3 CENP-A binding domain so wouldn't be able to perform CENP-A deposition. Therefore, smaller truncation mutagenesis of HJURP or, ideally, point mutant identification is necessary to nail down the contribution of HJURP decondensation to CENP-A deposition.

Does the condensin II complex reduce chromatin decondensation by HJURP and does it localize to centromeres in early G1 cells?

As displayed in Figure 4-2, LacI-HJ ${ }^{1-748}$ arrays appeared less expanded when CAP-H2 was recruited to the array. It would be informative to measure the array length to width ratios in LacI-HJ ${ }^{1-748}$ positive cells where CAP-H2-LAP is present at the array. If condensin II is countering HJURP's decondensation, then these arrays should be more condensed than LacI-HJ ${ }^{1-748}$ arrays without any over-expressed CAP-H2-LAP present.

Lastly, we need to determine if the condensin II complex is present at centromeres in early G1. It co-localizes with CENP-A during mitosis and is present in the nucleus throughout the cell cycle (Ono et al., 2004), but if we could demonstrate it overlaps in time with HJURP at centromeric repeats using ChIP or immunofluorescence, this would make our argument that it is required during the CENP-A deposition window more convincing.

Determine the role of Mis $18 \alpha$ in chromatin condensation 
The data presented in the appendix of this chapter demonstrate Mis $18 \alpha$ cotargeting can reverse HJURP's chromatin decondensation and that this required an intact CxxC domain (Appendix Figure 4-1). We want to understand if this is a direct effect of Mis $18 \alpha$ or if Mis18 $\alpha$ is indirectly influencing chromatin compaction. Mis18 could indirectly affect HJURP's ability to decondense chromatin by epigenetically altering the chromatin environment at the array or by recruiting $\mathrm{RbAp} 46 / 48$, a known interacting partner with the Mis 18 complex and member of chromatin remodeling complexes (Fujita et al., 2007).

Alternatively, Mis $18 \alpha$ could directly inhibit HJURP's chromatin decondensation activity. This argues that Mis $18 \alpha$ has inherent chromatin condensation activity. There is some published evidence suggesting this (Kim et al., 2012), but it has not been formally demonstrated. Additional in vitro pull down experiments determining which regions of the protein are required for its DNA binding activity will narrow down which regions may be involved in DNA compaction. Then, electromobility shift assays (EMSA) can be used to demonstrate Mis18 $\alpha$ condensing a plasmid in vitro. Additionally, it would be informative to determine if HJURP's ability to assemble CENP-A chromatin in vitro is hampered by pre-binding Mis $18 \alpha$ to the plasmid DNA template. 


\section{Materials and Methods}

siRNA, Western Blotting, and Q-PCR

U2OS-LacO or HeLa TRex cell lines stably expressing Mis18 $\alpha$-GFP, HJURP-LAP, or mCherry-CENP-A were plated at $1 \times 10^{5}$ cells in six-well plates, on polylysine-coated coverslips if used for immunofluorescence. If applicable, cells were transfected with Lipofectamine 2000 as described then were treated with siRNA following 8 hours of transfection. Transfection media was left in the well. For siRNA treatment alone without transfection, 24 hours after plating, cells were treated with siRNA. Concentrations in well (3 mL total volume in 6-well plate format) and product information: 50nM CAP-D3 custom Stealth siRNA from LifeTechnologies (5' CAA GCC UCU GUU AAC UUG AAU UCC U 3'), 33nM custom Stealth siRNA from LifeTechnologies CAP-H3 (5' UUC CAG AGA UGA AAU CAA GGG CCU G 3'), 20 nM HJURP Silencer Select siRNA from LifeTechnologies (siRNA ID s30814), or equal amount of Negative Control \#2 Silencer Select siRNA from LifeTechnologies (Catalog \# 4390846). RNAiMAX was used as lipofection reagent. After 24 hours, 1/3 of the plating volume DMEM with 10\% heat inactivated FBS $+5 \%$ Pen/Strep was added. For CAP-H2 + CAP-D3 depletion, existing media in the well was removed, and a second siRNA treatment was done at 48 hours after plating. For HJURP depletion western blot analysis, cells were harvested forty-eight hours after siRNA treatment with PBS + 3 mM EDTA, counted and whole-

cell lysates were made in SDS-PAGE sample buffer. Lysates from $1 \times 10^{5}$ cells per lane were separated on $10 \%$ SDS-PAGE gel and transferred to nitrocellulose. Blots were incubated in primary anti-HJURP (\#3399) or anti-tubulin (AA2) antibody overnight at 
$4^{\circ} \mathrm{C}$ and in secondary (Jackson Laboratories) for 1 hour RT. For CAP-H2 and CAP-D3 depletion analysis, cells were harvested using PBS $+3 \mathrm{mM}$ EDTA and were washed $1 \mathrm{x}$ with PBS. RNA was extracted using Qiagen RNeasy Mini Kit (Catalog \# 74104). cDNA library was prepared using 1 ug RNA as input for iScript cDNA synthesis kit (BioRad 1708890). Quantitative PCR with primers to GUS, CAP-H2, or CAP-D3 from $1 \mathrm{uL}$ cDNA template was performed (Sybr Green $\mathrm{iQ}^{\mathrm{TM}}$ SYBR ${ }^{\circledR}$ Green Supermix \#170-8880).

\section{Cell culture, transfections and immunocytochemistry}

HeLa or U2OS-LacO cells were plated to poly-lysine coated coverslips at $1 \times 10^{5}$ cells per well in 6 well, $0.6 \times 10^{5}$ cells per well in 24 -well plate, or $1 \times 10^{6}$ cells for a $10 \mathrm{~cm}^{2}$ plate. Cells were transfected in Optimem 24 hours later with 0.2-0.25 ug plasmid DNA (24well plate), 1ug (6-well plate), or 5.8ug (10 $\mathrm{cm}^{2}$ dish) using $0.4 \mathrm{uL}$ (24-well plate), $2 \mathrm{uL}$ (6-well plate), or 11.6uL (10 $\mathrm{cm}^{2}$ dish) Lipofectamine ${ }^{\mathrm{TM}} 2000$ (LifeTechnologies 11668027). Cells were left in Optimem + transfection complexes for 9 hours then media was changed to DMEM $+10 \%$ FBS, $5 \%$ Pen/Strep. HEK293T cells were transfected in serum-free, Pen/Strep-free DMEM using 4ug DNA, 30 uL Polyfect (Qiagen 301105) per $6 \mathrm{~cm}^{2}$ dish.

Live-cell imaging was conducted on the U2OS-LacO cell line at $37^{\circ} \mathrm{C}$ in Leibovitz L-15 media including 10\% FBS following 48 hrs of transfection. IPTG (Sigma) was washed in using syringe to a final concentration of $15 \mathrm{mM}$ in $600 \mathrm{uL}$ well. Images were collected at 6 minute intervals on a Deltavision Microscope equipped with a Weatherstation environmental chamber maintained at $37^{\circ} \mathrm{C}$.

For fixation, U2OS-LacO and HeLa-TRex cells were pre-extracted with $0.1 \%$ Triton-X in PHEM Buffer for 3 minutes, fixed with 4\% paraformaldehyde in PBS for 10 
minutes, and then quenched by addition of $100 \mathrm{mM}$ Tris- $\mathrm{HCl}, \mathrm{pH} 7.5$ for another 5 minutes at room temperature. Cells were blocked in $2 \% \mathrm{FBS}, 2 \% \mathrm{BSA}$ in $0.1 \%$ TritionPBS. Centromeres were visualized with a rabbit polyclonal anti-CENP-T antibody 1:2000 (\#3408) or monoclonal CENP-A at 1:1000 dilution (Abcam, ab13939). Endogenous HJURP was stained using rabbit polyclonal antibody (\#3399) at 1:1000. DNA was stained with 4',6-diamidino-2-phenylindole (DAPI, $0.2 \mathrm{mg} / \mathrm{ml}$ ). Donkey-antirabbit Cy5-conjugated (Jackson Laboratories, \#111175003) or Cy-3 or FITC-conjugated goat anti-mouse, or secondary antibodies were used for detection and coverslips were mounted with 4 uL ProlongGold (LifeTechnologies).

All micrograph images were collected using either a $60 \mathrm{x}$ or $100 \mathrm{x}$ oil-immersion Olympus objective lens (numerical aperture $=1.40$ ) on a DeltaVision deconvolution microscope using a Photometrics CoolSNAP $\mathrm{HQ}^{2}$ camera. Acquisition software used was SoftWoRX from Applied Precision. Images were deconvolved and presented as stacked images. All representative images within cell lines within figures were collected with identical exposure times and scaled equally. Intensities in live-cell and fixed images were analyzed using ImageJ. Details of each analysis method are provided in appropriate figure legends.

\section{Mitotic Chromosome Spreads}

U2OS-LacO cells were arrested overnight in $0.1 \mathrm{ug} / \mathrm{mL}$ Nocodazole in DMEM GlutaMAX media 32 hours after transfection. Mitotic cells were harvested using a transfer pipette to blow cells off the plate. Cells were spun down, washed in 1xPBS, and resuspended at $1 \times 10^{6}$ cells $/ \mathrm{mL}$ in a hypotonic solution (20 mM Hepes (pH 7.0), $1 \mathrm{mM}$ $\mathrm{MgCl}_{2}, 0.2 \mathrm{mM} \mathrm{CaCl}, 20 \mathrm{mM} \mathrm{KCl}, \mathrm{LPC}$, and $0.5 \mathrm{ug} / \mathrm{mL}$ nocodazole/colcemid). After 
$10 \mathrm{~min}$ in the hypotonic solution, cells were spun onto glass slides using a cytospin at $2000 \mathrm{rpm}$ for 4 minutes (30,000 cells/slide), immediately hydrated with 1xPBS, and then fixed and immunostained as described above. The anti-mouse CENP-A antibody was used at a 1:1000 dilution (Abcam, 13939).

\section{ChIP}

U2OS-LacO cells were transfected for 48 hours with mCherry-LacI-HJURP fragments using Lipofectamine 2000 in a $10 \mathrm{~cm}^{2}$ plate as described above. $2 \times 10 \mathrm{~cm}^{2}$ plates were used per condition. Cells were harvested using trypsin, washed $1 \mathrm{x}$ with PBS then resuspended in $900 \mathrm{uL}$ media. Cells were fixed by adding $100 \mathrm{uL} 11 \mathrm{x}$ fixation solution $(11.1 \%$ formaldehyde, 50mM HEPES, 100mM NaCl, 1mM EDTA, 0.5mM EGTA). Crosslinking reaction was quenched with $100 \mathrm{uL} 1.5 \mathrm{M}$ glycine. Cells were spun down, washed $1 \mathrm{x}$ with FACS buffer (2\% BSA, $0.05 \% \mathrm{NaN}_{3}$, PBS), spun down, resuspended in 200uL SDS lysis buffer (50mM Tris- $\mathrm{HCl} \mathrm{pH} 8.0,10 \mathrm{mM}$ EDTA pH 8.0, 1\% SDS, PMSF, LPC), then incubated on ice for 10 minutes. Lysates were then sonicated using Biorupter $(4 \times 10$ minute cycles of 30 seconds on, 30 seconds off). Rest on ice in between each cycle. Centrifuge max speed then transfer supernatent to a new tube. Dilute in $1300 \mathrm{uL}$ ChIP dilution buffer $(50 \mathrm{mM}$ Tris- $\mathrm{HCl} \mathrm{pH} 8.0,167 \mathrm{mM} \mathrm{NaCl}, 1.1 \%$ triton $\mathrm{x}-100,0.11 \%$ sodium deoxycholate, PMSF, LPC) add antibody (0.5ug/sample) and incubate with rotation overnight at $4^{\circ}$. The next day, add ProteinA Dynabeads $(10 \mathrm{uL} / \mathrm{sample})$ and incubate at $4^{\circ}$ with rotation for 3 hours. Wash beads in $500 \mathrm{uL}$ of following: 1x RIPA+150mM NaCl, 1x RIPA+500mM NaCl, $\mathrm{LiCl}$ wash solution $(10 \mathrm{mM}$ Tris- $\mathrm{HCl} \mathrm{pH} 8.0,0.25 \mathrm{M} \mathrm{LiCl}, 1 \mathrm{mM}$ EDTA, $0.5 \%$ NP-40, $0.5 \%$ sodium deoxycholate), then $2 x$ in TE. Resuspend beads in 200uL ChIP direct elution buffer (10mM Tris-HCl, 300mM NaCl, 5mM EDTA, $0.5 \%$ 
SDS) and incubate at $65^{\circ}$ overnight to reverse crosslinks. Isolate DNA following RNase and Proteinase $\mathrm{K}$ digestion by adding glycogen $(1 \mathrm{uL} 20 \mathrm{mg} / \mathrm{mL})$ then add $210 \mathrm{uL}$ phenol/CIAA and shake vigorously. Centrifuge max speed 10 minutes. Take sup. Backextract organic layer with $180 \mathrm{uL} \mathrm{TE}+200 \mathrm{mM} \mathrm{NaCl}$. Combine aqueous layers, add $900 \mathrm{uL}$ $100 \%$ ethanol and precipitate overnight at $-20^{\circ}$. Centrifuge samples max speed 20 minutes at $4^{\circ}$ then allow pellets to air dry. Resuspend ChIP'd DNA in 20uL DNase-free $\mathrm{H}_{2} \mathrm{O}$ or TE. Analyze $2 \mathrm{uL}$ by rt-PCR.

\section{Immunoprecipitations}

HEK293T cells were plated to $90 \%$ confluency in $6 \mathrm{~cm}^{2}$ dishes. After 24 hours, cells were transfected using Polyfect (Qiagen) as described above. For mitotically arrested population, $0.1 \mathrm{ug} / \mathrm{mL}$ nocodazole was added to the media for 12 hours. After 24 hours, cells were harvested on ice using PBS $+3 \mathrm{mM}$ EDTA. Cells were spun down then washed 1x with PBS. Cells were lysed for 10 minutes on ice in $1 \mathrm{~mL}$ of RIPA buffer with occasional vortexing $(150 \mathrm{mM} \mathrm{NaCl}, 1 \% \mathrm{NP}-40,0.3 \%$ deoxycholate, $0.15 \%$ SDS, $50 \mathrm{mM}$ Tris-HCl pH 7.5, 1mM EDTA, 10\% glycerol, 1x Roche protease inhibitors, 200uM NaV, $0.5 \mathrm{mM}$ PMSF, $5 \mathrm{mM} \mathrm{NaF}, 50 \mathrm{mM}$ beta-glycerophosphate). Lysates were sonicated $2 \times 10$ cycles $30 \mathrm{sec}$ on/30sec off using Biorupter. Lysates were spun down max speed then the full $1 \mathrm{~mL}$ supernatent was pre-cleared with $10 \mathrm{uL}$ Protein A agarose beads for 1 hour on ice. Beads were spun out and supernatents were transferred to a fresh tube and luL HA antibody (Covance HA.11 161312) or $0.2 \mathrm{uL}$ of $\mathrm{IgG}$ was added overnight at $4^{\circ}$ with rotation. The next day, 8uL Protein A Dynabeads (LifeTechnologies 10001D) were added for 1 hour on ice. Beads were washed 1x with RIPA buffer and 3x with PBST. Beads were resuspended in sample buffer. 


\section{Inducible CENP-A cell line}

HeLa-TRex cells containing a DOX-inducible mCherry-CENP-A integration were plated at $1 \times 10^{5}$ cells per well in a 6 -well plate, cells were plated on polylysine coated coverslips for immunofluorescence wells. Cells were kept in serum-free media during this protocol (following the initial plating step) to ensure mCherry-CENP-A was not expressed until Dox was added. After 24 hours, cells were treated with siRNA + RNAiMAX. See siRNA treatment described above for details. After 32 hours of siRNA treatment ( 8 hours following $2^{\text {nd }}$ round of siRNA, if applicable), Dox was added at a concentration of $1 \mathrm{ug} / \mathrm{mL}$ to induce the expression of the mCherry-CENP-A. siRNA was left in the well to ensure that depletion continued during CENP-A loading. Cells were induced with Dox overnight, then fixed and stained.

\section{Antibodies}

1. CENP-A mouse monoclonal (Abcam 13939), 1:1000, immunofluorescence

2. CENP-T rabbit polyclonal (\#3408), 1:2000, immunofluorescence

3. GFP rabbit polyclonal (\#3404), 1:1000, immunoblotting

4. HA mouse monoclonal (Covance HA.11 161312), 1:1000, immunoblotting

5. Npm1 mouse monoclonal, 1:10,000, immunoblotting

6. HJURP rabbit polyclonal (\#3399), 1:1000, immunofluorescence

7. MBP-HRP mouse monoclonal (NEB E8038S), 1:8000, immunoblotting

8. H3 pan rabbit polyclonal (Abcam 1791), $0.5 \mathrm{ug} / \mathrm{sample}$, ChIP. 
CHAPTER V: ADDITIONAL PUBLICATION CONTRIBUTIONS 
This work was done as a contribution to the following publication:

Bassett EA., DeNizio J., Barnhart-Dailey MC., Panchenko, T., Sekulic N., Rogers DJ., Foltz DR., Black BE. "HJURP uses distinct CENP-A surfaces to recognize and to stabilize CENP-A/Histone H4 for Centromere Assembly." Developmental Cell (2012), 22:749-762.

\section{HJURP uses distinct CENP-A surfaces to recognize and to stabilize CENP-}

\section{$\underline{\text { A/Histone } \mathrm{H} 4 \text { for centromere assembly }}$}

\section{Summary}

The region of the CENP-A nucleosome that was initially shown to mediate its specific recognition by its chaperone HJURP is termed the CENP-A targeting domain (CATD). If this portion of the CENP-A histone is swapped into the H3 histone, it is sufficient to mediate $\mathrm{H} 3^{\mathrm{CATD}}$ recognition by HJURP, which was required to target the H3 ${ }^{\text {CATD }}$ hybrid to the centromere (Black et al., 2004; Foltz et al., 2009). However, a crystal structure of the HJURP/CENP-A/H4 ternary complex was published after these studies that challenged the CATD's ability to direct CENP-A recognition by HJURP. In this study, a single amino acid in CENP-A (Serine 68) that resides outside of the CATD was shown to be the primary specificity determinant for HJURP recognition. This was verified by in vitro pull-down experiments demonstrating an $\mathrm{H} 3$ histone with its glutamine at position 68 changed to a serine could be recognized by HJURP (Hu et al., 2011).

Due to these contradictory findings, Bassett et al. undertook this study in order to definitively understand the role of the CATD in CENP-A nucleosome recognition by HJURP. Using the LacO/TRE U2OS cell line used in extensively in this work (Janicki et 
al., 2004), they demonstrate LacI-HJURP was unable to recruit the $\mathrm{H} 3^{\mathrm{Q} 68 \mathrm{~S}}$ chimera in vivo shown by Hu et al. in vitro to provide recognition specificity by HJURP. They also thoroughly characterized the requirement for the CENP-A CATD for HJURP interaction. They demonstrated that HJURP not only acts as a chaperone by preventing aberrant CENP-A association with DNA, but it also stabilized the histone folds of both CENP-A and H4. They lastly characterized that both a hydrophobic stitch in the CENP-A CATD $\mathrm{H} 4$ interface and an intact CENP-A/CENP-A dimerization interface are required for stable HJURP-mediated CENP-A nucleosome assembly (Bassett et al., 2012b).

My contribution to the work was looking at the stability of LacI-HJURPassembled CENP-A CATD mutants at the LacO/TRE array following IPTG addition (Figure 5-1). I additionally demonstrated Tryptophan 66 on HJURP was not required for stable CENP-A assembly by LacI-HJURP at the LacO array (Figure 5-2). Tryptophan 66 was the complementary interacting residue on HJURP for Serine 68 on CENP-A shown by $\mathrm{Hu}$ et al. to mediate binding specificity in vitro (Hu et al., 2011). Lastly, I used the $\mathrm{LacO} / \mathrm{TRE}$ array to show that the CENP-A/CENP-A dimerization interface was required for stable, HJURP-mediated deposition (Figure 5-2) (Bassett et al., 2012b). 
HJURP recognition of CENP-A is solely dependent on residues in the CATD

Five sets of mutations (called L1, $\alpha 2, \alpha 2.1, \alpha 2.2, \alpha 2.3$ ) where CATD residues are replaced with the corresponding residues from histone $\mathrm{H} 3$ each abolish efficient CENP-A targeting to centromeres (Black et al., 2004; Shelby et al., 1997), but it had not been tested whether or not any of these affect recognition by HJURP. Mutants are represented in Figure 5-1 C. Although both the loop 1 (L1) and $\alpha 2$ helix of CENP-A are in contact with HJURP in a crystal structure (Hu et al., 2011), Hu and colleagues concluded that there are no good candidate side-chain substitutions in this entire region of CENP-A where $\mathrm{H} 3$ residues would preclude binding. To directly test this proposal, we employed a cell-based approach that we recently described that monitors HJURP association at an ectopic site and HJURP-mediated stable assembly of CENP-A into chromatin (Barnhart et al., 2011). This approach utilizes the $\mathrm{LacO} / \mathrm{I}$ chromosome-tethering system, where LacI-fused proteins can be targeted to a chromosomally incorporated LacO array and subsequently removed by the addition of the LacI allosteric effector molecule, IPTG (Belmont, 2001; Janicki et al., 2004). Endogenous CENP-A is efficiently recruited to the HJURP-containing arrays (Barnhart et al., 2011). Importantly, the centromere targeting of CENP-A is independently measured within individual cells.

We found that four (L1, $\alpha 2.1, \alpha 2.2, \alpha 2.3)$ of the five mutants that fail to target to centromeres (Bassett et al., 2012b) are robustly recruited to HJURP-containing arrays, whereas the fifth ( $\alpha 2)$ fails to enrich at the HJURP-containing arrays (Figure 5-1 A, B). In addition, all four of the mutants that retain robust HJURP recognition and recruitment to the ectopic chromosomal array are stably incorporated into the ectopic chromosomal locus after LacI-HJURP removal (Figures 5-1 A, B), suggesting that their failure to target 
to centromeres is not due to an inability to undergo HJURP-mediated chromatin assembly. Perhaps the simplest explanation of these results is that the CATD provides the primary HJURP recognition determinants, and that a combination of CENP-A-specific residues spanning the entire $\alpha 2$ helix is required for HJURP binding. 
Figure 5-1. Characterization of CENP-A CATD mutant stability at the LacO/TRE array following LacI-HJURP removal

(A) Representative images of cells co-transfected with mCherry-LacI-HJURP, GFPTetR, and mutant CENP-A construct, and treated with or without $15 \mathrm{mM}$ IPTG for $1 \mathrm{~h}$ prior to processing for immunofluorescence. Quantification is shown in Fig. 1E. (B) Quantification of stable CENP-A incorporation into the HJURP-containing array. Cells were analyzed $48 \mathrm{hr}$ after co-transfection of mCherry-LacI-HJURP, GFP-TetR, and HAtagged CENP-A mutant proteins and a $1 \mathrm{hr}$ treatment with (gray) or without (black) 15 mM IPTG. (C) Sequences of the CENP-A mutants used in these experiments and a summary of our results. Black bars indicate residues shared in both H3 and CENP-A. 

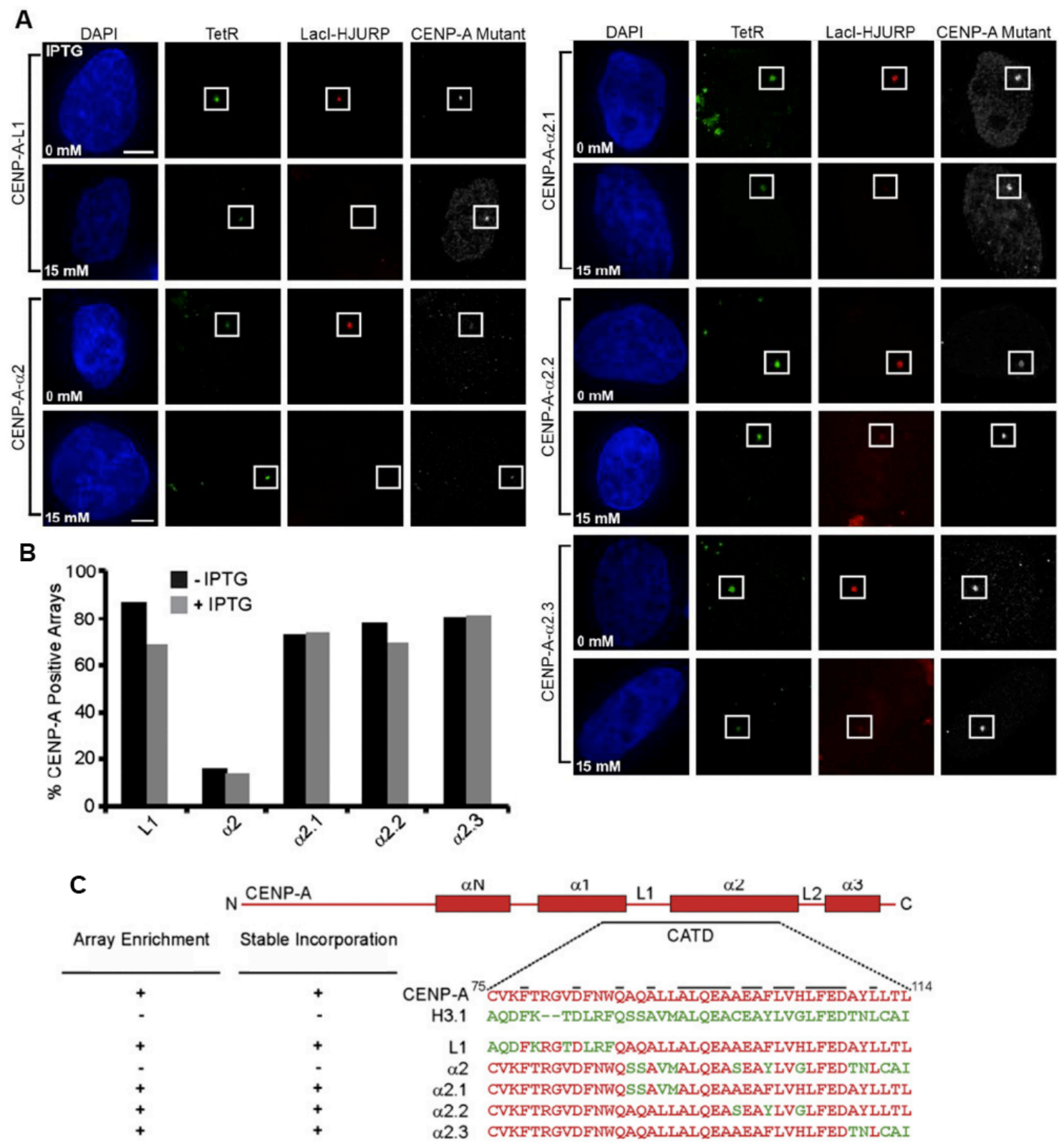


\section{HJURP W66 is not required for stable CENP-A deposition at the LacO/TRE array}

Based on the proposal of Hu and colleagues that information outside of the CATD provides the principal HJURP specificity determinant (Hu et al., 2011), Bassett et al. tested using the U2OS LacO/TRE cell based approach (Figure 5-1) the CENP-A ${ }^{\mathrm{S} 68 \mathrm{Q}}$ and $\mathrm{H} 3^{\mathrm{Q} 68 \mathrm{~S}}$ mutations that were reported in GST pull-down experiments to cause the two histone variants to switch allegiance: $\mathrm{CENP}-\mathrm{A}^{\mathrm{S} 68 \mathrm{Q}}$ was reported to eliminate HJURP binding, and $\mathrm{H}_{3}{ }^{\mathrm{Q} 68 \mathrm{~S}}$ was reported to confer HJURP binding (Hu et al., 2011). In contrast to the earlier GST pull-down experiments (Hu et al., 2011), Bassett et al. found that substitutions at this position (S68Q in CENP-A and Q68S in H3) had no measurable effect on centromere targeting or on recruitment to the HJURP-containing arrays: CENPA behaved the same as wild-type (WT) CENP-A and H3Q68S behaved the same as wildtype H3. These results in this cell-based test may indicate that the mutations (CENP$\mathrm{A}^{\mathrm{S} 68 \mathrm{Q}}$ and $\mathrm{H} 3^{\mathrm{Q} 68 \mathrm{~S}}$ ) behave differently in cells than in direct binding reactions as purified components and further supported that the CATD of CENP-A is the important recognition surface for HJURP (Bassett et al., 2012b).

In the crystal structure of the HJURP/CENP-A/H4 ternary complex, there is a hydrophobic pocket formed in a $\beta$ sheet portion of HJURP that accommodates the side chain of Serine 68 in CENP-A but where there is predicted unfavorable packing (particularly due to clashing with Tryptophan 66 of HJURP) with Glutamine 68 in the analogous position of $\mathrm{H} 3$ (Figure 5-2 C) (Hu et al., 2011). In order to assay for the requirement of this complementary binding residue in HJURP, we targeted a LacIHJURP fragment only containing amino acids 1-62 (missing Tryptophan 66) to the LacO/TRE array and assayed its ability to recruit and stably deposit CENP-A. We found 
that the small N-terminal portion of HJURP (aa 1-62) was nearly as efficient as the much larger domain of HJURP previously identified as sufficient (aa 1-208; Barnhart et al., 2011) in mediating the stable incorporation of endogenous CENP-A into an ectopic chromosomal locus (Figure 5-2 A, B). This shows that the complementary interacting residue, Tryptophan 66, of HJURP for recognizing the non-CATD residue Serine 68 of CENP-A is not necessary for stable HJURP-mediated CENP-A deposition (Bassett et al., 2012b).

Intact CENP-A/CENP-A Interface Is Required for Nucleosome Formation

CENP-A/H4 exists as a heterotetramer in solution with two copies of CENP-A (Black et al., 2004), and there are two copies of CENP-A in the available nucleosome crystal structure ((Tachiwana et al., 2011). Binding of HJURP/Scm3 occludes the CENPA/CENP-A interface (Cho and Harrison, 2011; Hu et al., 2011; Zhou et al., 2011), but HJURP assembles initial products in vitro that are nonetheless thought to include two copies of human CENP-A (or its yeast counterparts; (Barnhart et al., 2011; Dechassa et al., 2011; Mizuguchi et al., 2007; Shuaib et al., 2010; Xiao et al., 2011). Additionally, dimerization of HJURP is required for CENP-A assembly in human cells, and this is a possible mechanism by which two CENP-A molecules are delivered to the centromere at the same time (Zasadzinska et al., 2013). In any eukaryote, the composition of the centromeric nucleosome in vivo is far less clear, with proposed models including an octameric nucleosome containing two copies each of CENP-A/H4/H2A/H2B (mammals, budding yeast, and insects; (Camahort et al., 2007; Foltz et al., 2006; Sekulic et al., 2010; Shelby et al., 1997; Zhang et al., 2012)), a tetrasome containing two copies each of CENP-A/H4 (yeast; (Williams et al., 2009)), nucleosome like particles lacking H2A/H2B 
but retaining Scm3 after assembly into DNA (yeast; (Mizuguchi et al., 2007)), and, most radically, a hemisome containing one copy each of CENP-A/H4/H2A/H2B (insects; (Dalal et al., 2007)) that wraps DNA with the reverse handedness of conventional nucleosomes (insects and yeast; (Furuyama and Henikoff, 2009)).

In models including two copies of CENP-A, an intact CENP-A/CENP-A interface is key, whereas it would be unused and therefore dispensable in a hemisome with a single copy of CENP-A (Dalal et al., 2007; Furuyama and Henikoff, 2009). In (CENP-A/H4) heterotetramers that spontaneously form upon co-expression in bacteria or after stepwise assembly into nucleosomes, the two CENP-A chains are held together by hydrophobic interactions of several side chains (including those from Leu111, Leu128, and Ile132) and an intermolecular salt bridge between His115 on one chain and Asp125 on the other (Sekulic et al., 2010; Tachiwana et al., 2011) (Figure 5-2 F). In this way, the CENPA/CENP-A interface is held together in a nearly identical fashion as the $\mathrm{H} 3 / \mathrm{H} 3$ interface in the conventional nucleosome (Luger et al., 1997; Sekulic et al., 2010; Tachiwana et al., 2011). We designed a mutant version of CENP-A (CENP-A $\left.{ }^{\mathrm{H} 115 \mathrm{~A} / \mathrm{L} 128 \mathrm{~A}}\right)$ in which the salt bridge is broken, and the otherwise hydrophobic interface is weakened (Figure 5-2 F). Using the LacO/TRE cell-based approach, we found that the CENP-A dimer mutant was clearly enriched at the HJURP array (Figure 5-2 D, E). However, CENP-A ${ }^{\text {H115A/L128A }}$ failed to stably incorporate into chromatin at the array following IPTG treatment, with its targeting dependent on a persistent LacI-HJURP/LacO interaction (Figure 5-2 D, E). These data suggest a strong requirement for an intact CENP-A/CENP-A interface in HJURP-mediated chromatin assembly at an ectopic chromosome locus. 
Figure 5-2. HJURP W66 is not required for stable CENP-A deposition at the LacO/TRE array and the CENP-A/CENP-A dimerization interface is required for stable CENP-A deposition by HJURP at the LacO/TRE array

(A) Representative images of stable incorporation of endogenous CENP-A into the HJURP ${ }^{1-62}$-containing array. (B) Quantification of stable incorporation of endogenous CENP-A into the HJURP-containing array. Cells co-transfected with mCherry-LacIHJURP $^{1-62}$ and GFP-TetR were treated $48 \mathrm{hr}$ post-transfection with (gray) or without (black) 15mMIPTG for $1 \mathrm{hr}$, and assessed for recruitment of endogenous CENP-A to the array. The values shown are normalized to the level of CENP-A recruitment to mCherry-

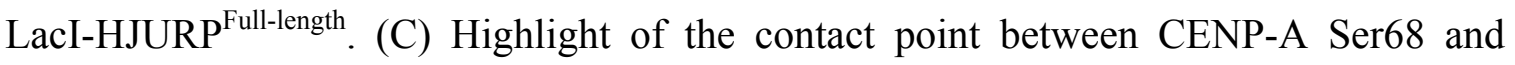
HJURP Trp66 (PDB 3R45; (Hu et al., 2011)). The region of HJURP deleted in the HJURP $^{1-62}$ version is labeled in black. (D) Representative images of cells co-transfected with mCherry-LacI-HJURP, GFP-TetR, and HA-tagged CENP-A ${ }^{\text {H115A/L128A }}$ and treated with or without IPTG for $1 \mathrm{hr}$ prior to processing for immunofluorescence. The boxes indicate the location of the LacO array identified with TetR. Scale bar, $5 \mu \mathrm{m}$. (E) Quantification of CENP-A ${ }^{\mathrm{H} 115 \mathrm{~A} / \mathrm{L} 128 \mathrm{~A}}$ stable incorporation into the HJURP-containing array with (gray) or without (black) $15 \mathrm{mM}$ IPTG. At least 30 cells were counted over multiple experiments. (F) Structure of the CENP-A nucleosome (PDB 3AN2; Tachiwana et al., 2011), with inset depicting residues H115 and L128 within the CENP-A/CENP-A interface. 
A
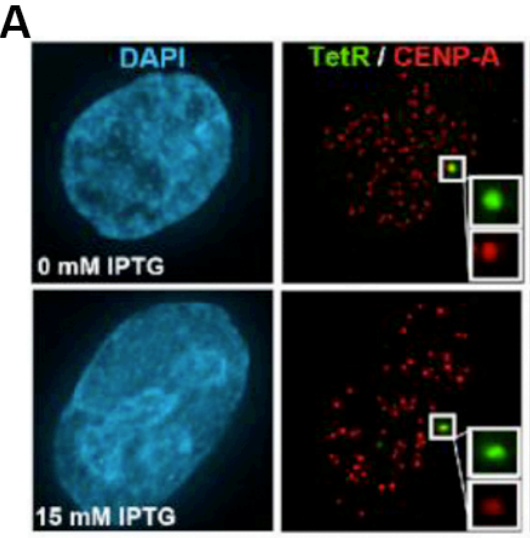

C

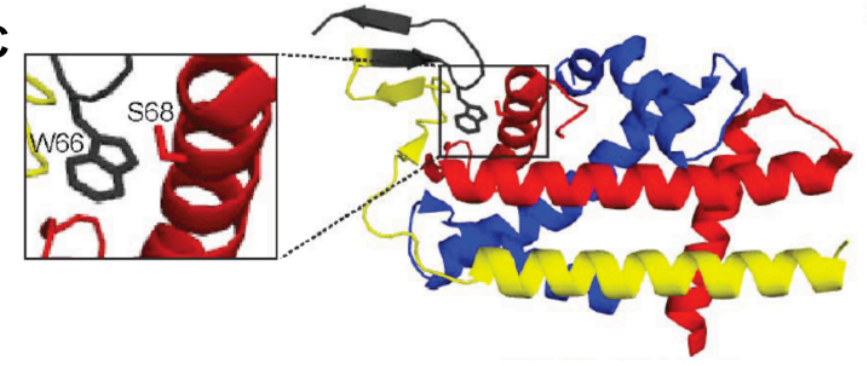

HJURP/CENP-A/H4
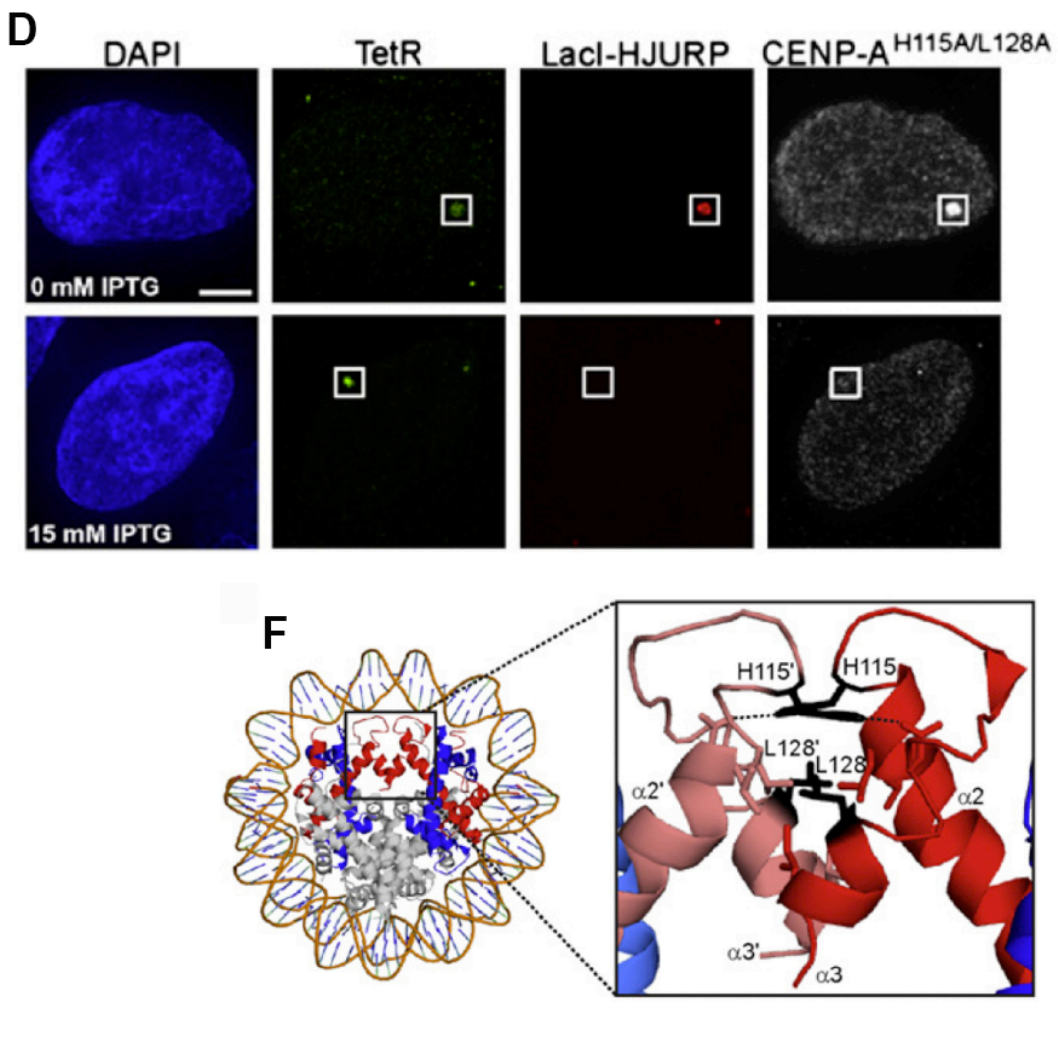

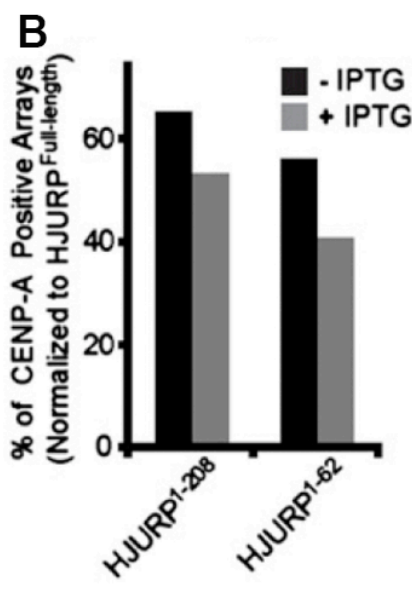


This work was done as a contribution to the following publication:

Zasadzinska E., Barnhart-Dailey MC., Kuich PHJL., Foltz DR. "Dimerization of the CENP-A assembly factor HJURP is required for centromeric nucleosome deposition." EMBO (2013), 32(15):2113-2124.

\section{Dimerization of the CENP-A assembly factor HJURP is required for}

\section{$\underline{\text { centromeric nucleosome deposition }}$}

Vertebrate HJURP proteins are significantly larger than their yeast orthologues and contain additional conserved domains (CDs) (Sanchez-Pulido et al, 2009). Human HJURP contains two HJURP_C-terminal domains (HCTD) within the carboxyl terminal half of the protein. A study by Ewelina Zasadzinska in the Foltz lab investigated the function of these HCTD's in human HJURP. The first HCTD was identified as the centromere targeting region of HJURP. The second HCTD was shown to mediate HJURP self-dimerization in the pre-nucleosomal complex. This self-dimerization was required for CENP-A deposition and provided a mechanism by which HJURP could assemble two heterodimers of CENP-A/H4 into a heterotetramer of (CENP-A/H4) $)_{2}$ to be incorporated into the octameric CENP-A nucleosome (Tachiwana et al., 2011; Zasadzinska et al., 2013). My contribution to the work showed in vivo self-association of the HJURP Cterminus using the LacO/TRE targeting system (Figure 5-3). 
In vivo dimerization of HJURP through the carboxyl terminus

The ability of HJURP to self-associate was examined using a LacI/LacO-based in vivo interaction assay to directly assess if HJURP multimerizes in vivo (Figure 5-3 A). Full length or HJURP ${ }^{352-e n d}$ was fused to the lac repressor (LacI) and expressed as bait in cells that have a stably integrated LacO array (Barnhart et al., 2011; Janicki et al., 2004). The interaction between HJURP proteins was tested by expressing GFP-HJURP fragments as prey. Tethering HJURP to the LacO array resulted in GFP-HJURP recruitment (Figure 5-3 B and C). Furthermore, LacI-HJURP was able to recruit carboxyl-terminal fragments of HJURP (Figure 5-3 B and C). This interaction only required the HJURP carboxyl terminus because tethering the LacI-HJURP ${ }^{352-e n d}$ fragment to the array was sufficient to recruit GFP-carboxyl fragments, containing amino acids 352-end and 482-end (Figure 5-3 D and E). Full length and HJURP ${ }^{352-\text { end }}$ showed minimal recruitment of an HJURP fragment containing amino acids 1-482 to the array, showing that HCTD2 in the carboxyl terminus is the primary site of HJURP self-association. The HCTD1 domain present in HJURP ${ }^{348-555}$ was unable to be efficiently recruited by either the full-length or HJURP ${ }^{352-e n d}$ bait protein (Figure 5-3 B-E). We conclude that the Cterminal region of HJURP containing the second HCTD2 domain of HJURP is sufficient to mediate self-interaction in vivo (Zasadzinska et al., 2013). 
Figure 5-3 In vivo recruitment of HJURP through the carboxyl terminus.

(A) Schematic of LacO-LacI interaction assay and the bait and prey constructs used in the study. (B) U2OS-LacO cells were co-transfected with mCherry-LacI-HJURP ${ }^{\text {FullLength }}$ and indicated GFP-tagged prey fragments. DNA is stained with DAPI. Centromere staining and endogenous CENP-A recruitment to the arrays are shown using anti-CENP-A antibody. Scale bar represents $5 \mu \mathrm{m}$. All images are scaled equally. Boxed regions are magnified to the right of merged images. (C) Quantification of prey protein recruitment to the array when HJURP ${ }^{\text {FullLength }}$ (grey) or control mCherry-LacI alone (black) is targeted. Recruitment is expressed as the ratio of GFP to mCherry integrated intensity at the array. (D) Cells co-transfected with mCherry-LacI-HJURP ${ }^{352-e n d}$ or mCherry-LacI alone as bait with the indicated GFP-tagged prey fragments. Cells were stained with DAPI to visualize DNA and anti-CENPA as in (B). Scale bar represents $5 \mu \mathrm{m}$. (E) Prey protein recruitment to the array in response to mCherry-LacI-HJURP ${ }^{352-\text { end }}$ or control mCherry-LacI targeting is quantified as in (C). The GFP:mCherry ratios for LacI-HJURP are plotted as the mean of $n=3$ experiments at $\geq 30$ arrays per condition. Error bars represent standard deviation. 
A
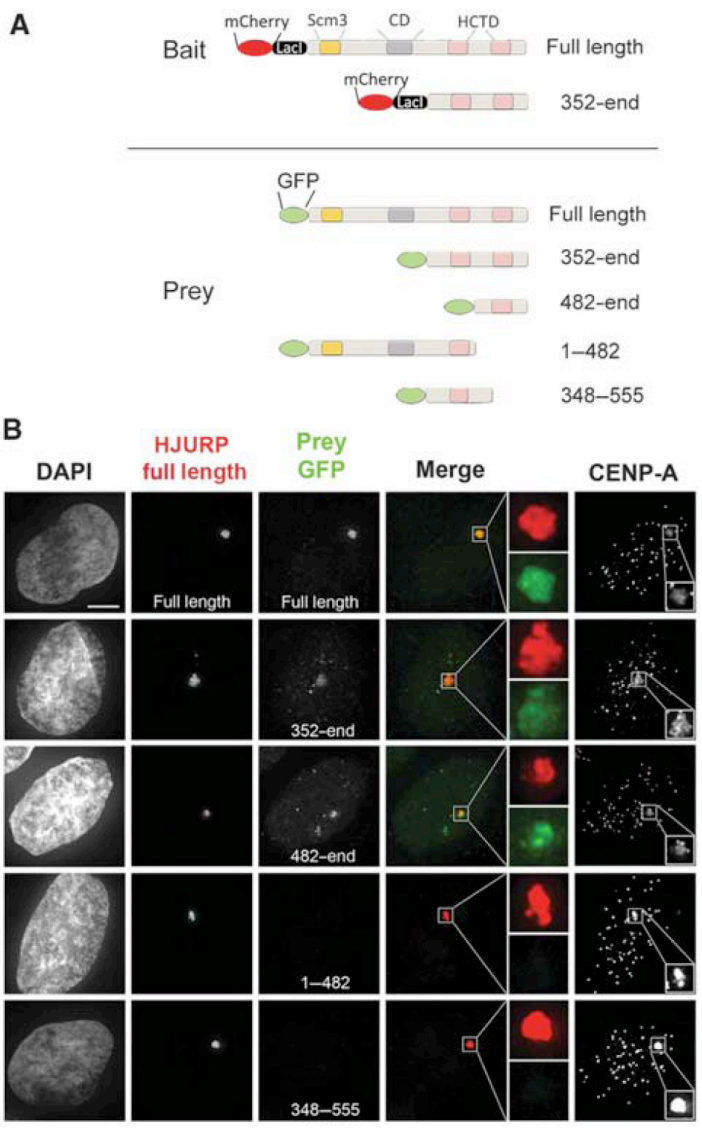

C $\quad 0.87] \quad$ Bait

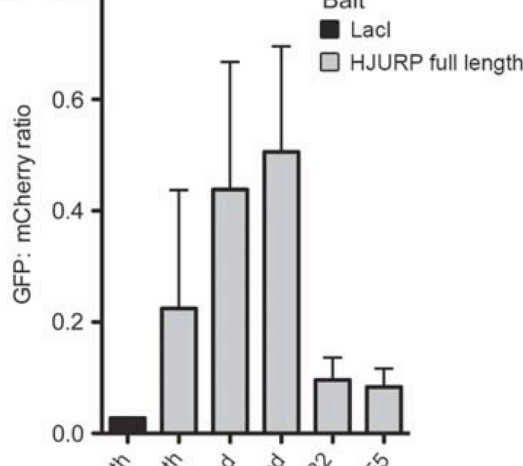

Prey

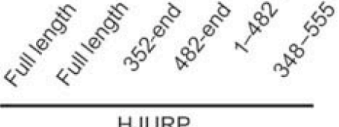

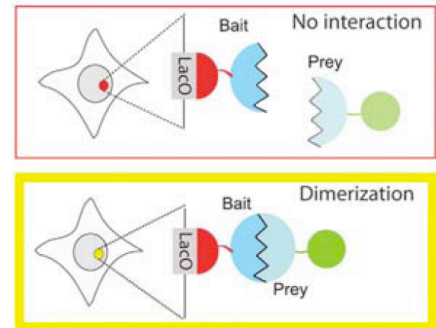
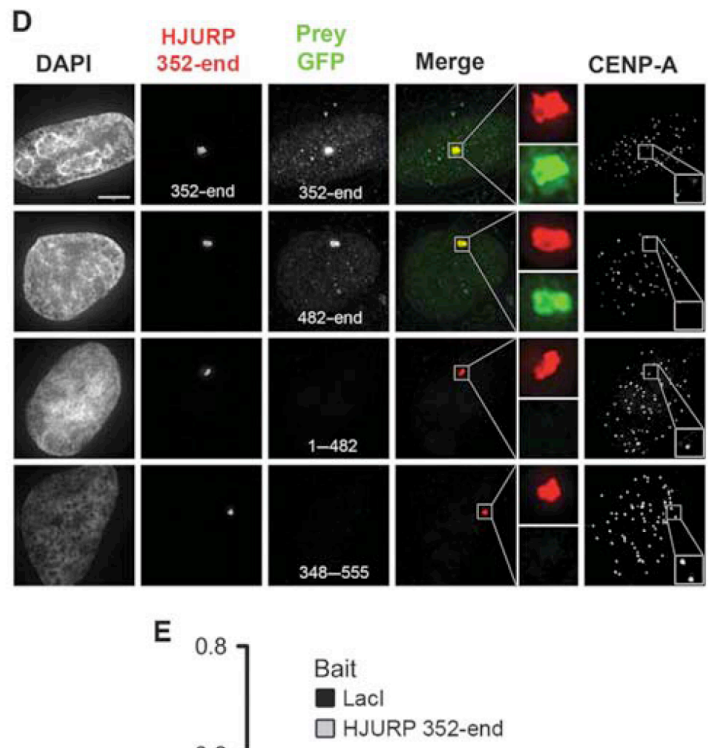

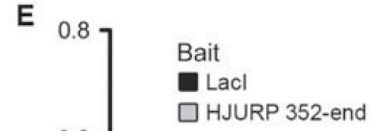

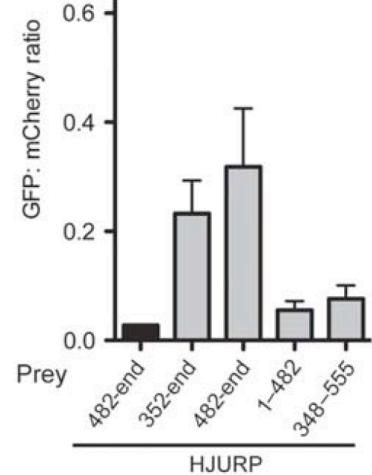




\section{References List}

Allshire, R.C., and Karpen, G.H. (2008). Epigenetic regulation of centromeric chromatin: old dogs, new tricks? Nat Rev Genet 9, 923-937.

Amaro, A.C., Samora, C.P., Holtackers, R., Wang, E., Kingston, I.J., Alonso, M., Lampson, M., McAinsh, A.D., and Meraldi, P. (2010). Molecular control of kinetochoremicrotubule dynamics and chromosome oscillations. Nat Cell Biol 12, 319-329.

Aravind, L., Iyer, L.M., and Wu, C. (2007). Domain architectures of the Scm3p protein provide insights into centromere function and evolution. Cell Cycle 6, 2511-2515.

Bailey, A.O., Panchenko, T., Sathyan, K.M., Petkowski, J.J., Pai, P.J., Bai, D.L., Russell, D.H., Macara, I.G., Shabanowitz, J., Hunt, D.F., et al. (2013). Posttranslational modification of CENP-A influences the conformation of centromeric chromatin. Proc Natl Acad Sci U S A 110, 11827-11832.

Barnhart, M.C., Kuich, P.H., Stellfox, M.E., Ward, J.A., Bassett, E.A., Black, B.E., and Foltz, D.R. (2011). HJURP is a CENP-A chromatin assembly factor sufficient to form a functional de novo kinetochore. J Cell Biol 194, 229-243.

Bassett, E.A., DeNizio, J., Barnhart-Dailey, M.C., Panchenko, T., Sekulic, N., Rogers, D.J., Foltz, D.R., and Black, B.E. (2012a). HJURP uses distinct CENP-A surfaces to recognize and to stabilize CENP-A/histone H4 for centromere assembly. Dev Cell 22, $749-762$.

Bassett, E.A., Denizio, J., Barnhart-Dailey, M.C., Panchenko, T., Sekulic, N., Rogers, D.J., Foltz, D.R., and Black, B.E. (2012b). HJURP Uses Distinct CENP-A Surfaces to Recognize and to Stabilize CENP-A/Histone H4 for Centromere Assembly. Dev Cell.

Belmont, A.S. (2001). Visualizing chromosome dynamics with GFP. Trends Cell Biol $11,250-257$.

Bergmann, J.H., Jakubsche, J.N., Martins, N.M., Kagansky, A., Nakano, M., Kimura, H., Kelly, D.A., Turner, B.M., Masumoto, H., Larionov, V., et al. (2012). Epigenetic engineering: histone $\mathrm{H} 3 \mathrm{~K} 9$ acetylation is compatible with kinetochore structure and function. J Cell Sci 125, 411-421. 
Bergmann, J.H., Rodriguez, M.G., Martins, N.M., Kimura, H., Kelly, D.A., Masumoto, H., Larionov, V., Jansen, L.E., and Earnshaw, W.C. (2011). Epigenetic engineering shows $\mathrm{H} 3 \mathrm{~K} 4 \mathrm{me} 2$ is required for HJURP targeting and CENP-A assembly on a synthetic human kinetochore. EMBO J 30, 328-340.

Bernad, R., Sanchez, P., Rivera, T., Rodriguez-Corsino, M., Boyarchuk, E., Vassias, I., Ray-Gallet, D., Arnaoutov, A., Dasso, M., Almouzni, G., et al. (2011). Xenopus HJURP and condensin II are required for CENP-A assembly. J Cell Biol 192, 569-582.

Black, B.E., Brock, M.A., Bedard, S., Woods, V.L., Jr., and Cleveland, D.W. (2007a). An epigenetic mark generated by the incorporation of CENP-A into centromeric nucleosomes. Proc Natl Acad Sci U S A 104, 5008-5013.

Black, B.E., Foltz, D.R., Chakravarthy, S., Luger, K., Woods, V.L., Jr., and Cleveland, D.W. (2004). Structural determinants for generating centromeric chromatin. Nature 430, 578-582.

Black, B.E., Jansen, L.E., Maddox, P.S., Foltz, D.R., Desai, A.B., Shah, J.V., and Cleveland, D.W. (2007b). Centromere identity maintained by nucleosomes assembled with histone $\mathrm{H} 3$ containing the CENP-A targeting domain. Mol Cell 25, 309-322.

Blower, M.D., Sullivan, B.A., and Karpen, G.H. (2002). Conserved organization of centromeric chromatin in flies and humans. Dev Cell 2, 319-330.

Bond, C.S., and Fox, A.H. (2009). Paraspeckles: nuclear bodies built on long noncoding RNA. J Cell Biol 186, 637-644.

Brinkley, B.R., and Cartwright, J., Jr. (1975). Cold-labile and cold-stable microtubules in the mitotic spindle of mammalian cells. Ann N Y Acad Sci 253, 428-439.

Burrack, L.S., and Berman, J. (2012). Neocentromeres and epigenetically inherited features of centromeres. Chromosome Res 20, 607-619.

Camahort, R., Li, B., Florens, L., Swanson, S.K., Washburn, M.P., and Gerton, J.L. (2007). $\mathrm{Scm} 3$ is essential to recruit the histone $\mathrm{h} 3$ variant cse4 to centromeres and to maintain a functional kinetochore. Mol Cell 26, 853-865. 
Camahort, R., Shivaraju, M., Mattingly, M., Li, B., Nakanishi, S., Zhu, D., Shilatifard, A., Workman, J.L., and Gerton, J.L. (2009). Cse4 is part of an octameric nucleosome in budding yeast. Mol Cell 35, 794-805.

Carone, D.M., Longo, M.S., Ferreri, G.C., Hall, L., Harris, M., Shook, N., Bulazel, K.V., Carone, B.R., Obergfell, C., O'Neill, M.J., et al. (2009). A new class of retroviral and satellite encoded small RNAs emanates from mammalian centromeres. Chromosoma $118,113-125$.

Carroll, C.W., Milks, K.J., and Straight, A.F. (2010). Dual recognition of CENP-A nucleosomes is required for centromere assembly. J Cell Biol 189, 1143-1155.

Carroll, C.W., Silva, M.C., Godek, K.M., Jansen, L.E., and Straight, A.F. (2009). Centromere assembly requires the direct recognition of CENP-A nucleosomes by CENPN. Nat Cell Biol 11, 896-902.

Chan, F.L., Marshall, O.J., Saffery, R., Kim, B.W., Earle, E., Choo, K.H., and Wong, L.H. (2012). Active transcription and essential role of RNA polymerase II at the centromere during mitosis. Proc Natl Acad Sci U S A 109, 1979-1984.

Chan, F.L., and Wong, L.H. (2012). Transcription in the maintenance of centromere chromatin identity. Nucleic Acids Res 40, 11178-11188.

Cheeseman, I.M., Hori, T., Fukagawa, T., and Desai, A. (2008). KNL1 and the CENP$\mathrm{H} / \mathrm{I} / \mathrm{K}$ complex coordinately direct kinetochore assembly in vertebrates. Mol Biol Cell 19, 587-594.

Chen, C.C., Dechassa, M.L., Bettini, E., Ledoux, M.B., Belisario, C., Heun, P., Luger, K., and Mellone, B.G. (2014). CAL1 is the Drosophila CENP-A assembly factor. J Cell Biol 204, 313-329.

Cho, U.S., and Harrison, S.C. (2011). Recognition of the centromere-specific histone Cse4 by the chaperone Scm3. Proc Natl Acad Sci U S A 108, 9367-9371.

Chueh, A.C., Northrop, E.L., Brettingham-Moore, K.H., Choo, K.H., and Wong, L.H. (2009). LINE retrotransposon RNA is an essential structural and functional epigenetic component of a core neocentromeric chromatin. PLoS Genet 5, e1000354. 
Chun, Y., Park, B., Koh, W., Lee, S., Cheon, Y., Kim, R., and Che, L. (2011). New centromeric component $\mathrm{CENP}-\mathrm{W}$ is an RNA-associated nuclear matrix protein that interacts with nucleophosmin/B23 protein. J Biol Chem 286, 42758-42769.

Cleveland, D.W., Mao, Y., and Sullivan, K.F. (2003). Centromeres and kinetochores: from epigenetics to mitotic checkpoint signaling. Cell 112, 407-421.

Collins, K.A., Camahort, R., Seidel, C., Gerton, J.L., and Biggins, S. (2007). The overexpression of a Saccharomyces cerevisiae centromeric histone H3 variant mutant protein leads to a defect in kinetochore biorientation. Genetics 175, 513-525.

Dalal, Y., Wang, H., Lindsay, S., and Henikoff, S. (2007). Tetrameric structure of centromeric nucleosomes in interphase Drosophila cells. PLoS Biol 5, e218.

Dambacher, S., Deng, W., Hahn, M., Sadic, D., Frohlich, J., Nuber, A., Hoischen, C., Diekmann, S., Leonhardt, H., and Schotta, G. (2012). CENP-C facilitates the recruitment of M18BP1 to centromeric chromatin. Nucleus 3, 101-110.

Dechassa, M.L., Wyns, K., Li, M., Hall, M.A., Wang, M.D., and Luger, K. (2011). Structure and Scm3-mediated assembly of budding yeast centromeric nucleosomes. Nat Commun 2, 313 .

Dimitriadis, E.K., Weber, C., Gill, R.K., Diekmann, S., and Dalal, Y. (2010). Tetrameric organization of vertebrate centromeric nucleosomes. Proc Natl Acad Sci U S A 107, 20317-20322.

Dornblut, C., Quinn, N., Monajambashi, S., Prendergast, L., van Vuuren, C., Munch, S., Deng, W., Leonhardt, H., Cardoso, M.C., Hoischen, C., et al. (2014). A CENP-S/X complex assembles at the centromere in $\mathrm{S}$ and $\mathrm{G} 2$ phases of the human cell cycle. Open Biol 4, 130229.

Drane, P., Ouararhni, K., Depaux, A., Shuaib, M., and Hamiche, A. (2010). The deathassociated protein DAXX is a novel histone chaperone involved in the replicationindependent deposition of H3.3. Genes Dev 24, 1253-1265.

Du, Y., Topp, C.N., and Dawe, R.K. (2010). DNA binding of centromere protein C (CENPC) is stabilized by single-stranded RNA. PLoS Genet 6, e1000835. 
Dunleavy, E.M., Almouzni, G., and Karpen, G.H. (2011). H3.3 is deposited at centromeres in $\mathrm{S}$ phase as a placeholder for newly assembled CENP-A in G(1) phase. Nucleus 2, 146-157.

Dunleavy, E.M., Pidoux, A.L., Monet, M., Bonilla, C., Richardson, W., Hamilton, G.L., Ekwall, K., McLaughlin, P.J., and Allshire, R.C. (2007). A NASP (N1/N2)-related protein, Sim3, binds CENP-A and is required for its deposition at fission yeast centromeres. Mol Cell 28, 1029-1044.

Dunleavy, E.M., Roche, D., Tagami, H., Lacoste, N., Ray-Gallet, D., Nakamura, Y., Daigo, Y., Nakatani, Y., and Almouzni-Pettinotti, G. (2009). HJURP is a cell-cycledependent maintenance and deposition factor of CENP-A at centromeres. Cell 137, 485497.

Earnshaw, W.C., and Rothfield, N. (1985). Identification of a family of human centromere proteins using autoimmune sera from patients with scleroderma. Chromosoma 91, 313-321.

Eickbush, T.H. (1992). Transposing without ends: the non-LTR retrotransposable elements. New Biol 4, 430-440.

Erhardt, S., Mellone, B.G., Betts, C.M., Zhang, W., Karpen, G.H., and Straight, A.F. (2008). Genome-wide analysis reveals a cell cycle-dependent mechanism controlling centromere propagation. J Cell Biol 183, 805-818.

Espelin, C.W., Kaplan, K.B., and Sorger, P.K. (1997). Probing the architecture of a simple kinetochore using DNA-protein crosslinking. J Cell Biol 139, 1383-1396.

Ferri, F., Bouzinba-Segard, H., Velasco, G., Hube, F., and Francastel, C. (2009). Noncoding murine centromeric transcripts associate with and potentiate Aurora B kinase. Nucleic Acids Res 37, 5071-5080.

Folco, H.D., Pidoux, A.L., Urano, T., and Allshire, R.C. (2008). Heterochromatin and RNAi are required to establish CENP-A chromatin at centromeres. Science 319, 94-97.

Foltz, D.R., Jansen, L.E., Bailey, A.O., Yates, J.R., 3rd, Bassett, E.A., Wood, S., Black, B.E., and Cleveland, D.W. (2009). Centromere-specific assembly of CENP-a nucleosomes is mediated by HJURP. Cell 137, 472-484. 
Foltz, D.R., Jansen, L.E., Black, B.E., Bailey, A.O., Yates, J.R., 3rd, and Cleveland, D.W. (2006). The human CENP-A centromeric nucleosome-associated complex. Nat Cell Biol 8, 458-469.

Foltz, D.R., and Stukenberg, P.T. (2012). A new histone at the centromere? Cell 148, 394-396.

Frehlick, L.J., Eirin-Lopez, J.M., and Ausio, J. (2007). New insights into the nucleophosmin/nucleoplasmin family of nuclear chaperones. Bioessays 29, 49-59.

Fujita, Y., Hayashi, T., Kiyomitsu, T., Toyoda, Y., Kokubu, A., Obuse, C., and Yanagida, M. (2007). Priming of centromere for CENP-A recruitment by human hMis18alpha, hMis18beta, and M18BP1. Dev Cell 12, 17-30.

Fukagawa, T., Nogami, M., Yoshikawa, M., Ikeno, M., Okazaki, T., Takami, Y., Nakayama, T., and Oshimura, M. (2004). Dicer is essential for formation of the heterochromatin structure in vertebrate cells. Nat Cell Biol 6, 784-791.

Furuyama, T., Dalal, Y., and Henikoff, S. (2006). Chaperone-mediated assembly of centromeric chromatin in vitro. Proc Natl Acad Sci U S A 103, 6172-6177.

Furuyama, T., and Henikoff, S. (2009). Centromeric nucleosomes induce positive DNA supercoils. Cell 138, 104-113.

Gascoigne, K.E., Takeuchi, K., Suzuki, A., Hori, T., Fukagawa, T., and Cheeseman, I.M. (2011). Induced ectopic kinetochore assembly bypasses the requirement for CENP-A nucleosomes. Cell 145, 410-422.

Gavet, O., and Pines, J. (2010a). Activation of cyclin B1-Cdk1 synchronizes events in the nucleus and the cytoplasm at mitosis. J Cell Biol 189, 247-259.

Gavet, O., and Pines, J. (2010b). Progressive activation of CyclinB1-Cdk1 coordinates entry to mitosis. Dev Cell 18, 533-543.

Gent, J.I., and Dawe, R.K. (2012). RNA as a structural and regulatory component of the centromere. Annu Rev Genet 46, 443-453. 
Glover, D.M. (2012). The overlooked greatwall: a new perspective on mitotic control. Open Biol 2, 120023.

Goldberg, A.D., Banaszynski, L.A., Noh, K.M., Lewis, P.W., Elsaesser, S.J., Stadler, S., Dewell, S., Law, M., Guo, X., Li, X., et al. (2010). Distinct factors control histone variant H3.3 localization at specific genomic regions. Cell 140, 678-691.

Gopalakrishnan, S., Sullivan, B.A., Trazzi, S., Della Valle, G., and Robertson, K.D. (2009). DNMT3B interacts with constitutive centromere protein CENP-C to modulate DNA methylation and the histone code at centromeric regions. Hum Mol Genet 18, 31783193.

Guse, A., Carroll, C.W., Moree, B., Fuller, C.J., and Straight, A.F. (2011). In vitro centromere and kinetochore assembly on defined chromatin templates. Nature 477, 354358.

Hasson, D., Panchenko, T., Salimian, K.J., Salman, M.U., Sekulic, N., Alonso, A., Warburton, P.E., and Black, B.E. (2013). The octamer is the major form of CENP-A nucleosomes at human centromeres. Nat Struct Mol Biol 20, 687-695.

Hayashi, T., Fujita, Y., Iwasaki, O., Adachi, Y., Takahashi, K., and Yanagida, M. (2004). Mis16 and Mis18 are required for CENP-A loading and histone deacetylation at centromeres. Cell 118, 715-729.

Hellwig, D., Emmerth, S., Ulbricht, T., Doring, V., Hoischen, C., Martin, R., Samora, C.P., McAinsh, A.D., Carroll, C.W., Straight, A.F., et al. (2011). Dynamics of CENP-N kinetochore binding during the cell cycle. J Cell Sci 124, 3871-3883.

Hemmerich, P., Weidtkamp-Peters, S., Hoischen, C., Schmiedeberg, L., Erliandri, I., and Diekmann, S. (2008). Dynamics of inner kinetochore assembly and maintenance in living cells. J Cell Biol 180, 1101-1114.

Heun, P., Erhardt, S., Blower, M.D., Weiss, S., Skora, A.D., and Karpen, G.H. (2006). Mislocalization of the Drosophila centromere-specific histone CID promotes formation of functional ectopic kinetochores. Dev Cell 10, 303-315.

Hewawasam, G., Shivaraju, M., Mattingly, M., Venkatesh, S., Martin-Brown, S., Florens, L., Workman, J.L., and Gerton, J.L. (2010). Psh1 is an E3 ubiquitin ligase that targets the centromeric histone variant Cse4. Mol Cell 40, 444-454. 
Hirano, M., and Hirano, T. (2006). Opening closed arms: long-distance activation of SMC ATPase by hinge-DNA interactions. Mol Cell 21, 175-186.

Hirano, T. (2012). Condensins: universal organizers of chromosomes with diverse functions. Genes Dev 26, 1659-1678.

Hori, T., Amano, M., Suzuki, A., Backer, C.B., Welburn, J.P., Dong, Y., McEwen, B.F., Shang, W.H., Suzuki, E., Okawa, K., et al. (2008a). CCAN makes multiple contacts with centromeric DNA to provide distinct pathways to the outer kinetochore. Cell 135, 10391052 .

Hori, T., Okada, M., Maenaka, K., and Fukagawa, T. (2008b). CENP-O class proteins form a stable complex and are required for proper kinetochore function. Mol Biol Cell $19,843-854$.

Hori, T., Shang, W.H., Takeuchi, K., and Fukagawa, T. (2013). The CCAN recruits CENP-A to the centromere and forms the structural core for kinetochore assembly. $\mathrm{J}$ Cell Biol 200, 45-60.

Hu, H., Liu, Y., Wang, M., Fang, J., Huang, H., Yang, N., Li, Y., Wang, J., Yao, X., Shi, Y., et al. (2011). Structure of a CENP-A-histone H4 heterodimer in complex with chaperone HJURP. Genes Dev 25, 901-906.

Ishii, K., Ogiyama, Y., Chikashige, Y., Soejima, S., Masuda, F., Kakuma, T., Hiraoka, Y., and Takahashi, K. (2008). Heterochromatin integrity affects chromosome reorganization after centromere dysfunction. Science 321, 1088-1091.

Izuta, H., Ikeno, M., Suzuki, N., Tomonaga, T., Nozaki, N., Obuse, C., Kisu, Y., Goshima, N., Nomura, F., Nomura, N., et al. (2006). Comprehensive analysis of the ICEN (Interphase Centromere Complex) components enriched in the CENP-A chromatin of human cells. Genes Cells 11, 673-684.

Janicki, S.M., Tsukamoto, T., Salghetti, S.E., Tansey, W.P., Sachidanandam, R., Prasanth, K.V., Ried, T., Shav-Tal, Y., Bertrand, E., Singer, R.H., et al. (2004). From silencing to gene expression: real-time analysis in single cells. Cell 116, 683-698.

Jansen, L.E., Black, B.E., Foltz, D.R., and Cleveland, D.W. (2007). Propagation of centromeric chromatin requires exit from mitosis. J Cell Biol 176, 795-805. 
Kagansky, A., Folco, H.D., Almeida, R., Pidoux, A.L., Boukaba, A., Simmer, F., Urano, T., Hamilton, G.L., and Allshire, R.C. (2009). Synthetic heterochromatin bypasses RNAi and centromeric repeats to establish functional centromeres. Science 324, 1716-1719.

Kato, T., Sato, N., Hayama, S., Yamabuki, T., Ito, T., Miyamoto, M., Kondo, S., Nakamura, Y., and Daigo, Y. (2007). Activation of Holliday junction recognizing protein involved in the chromosomal stability and immortality of cancer cells. Cancer Res 67, 8544-8553.

Keller, C., and Buhler, M. (2013). Chromatin-associated ncRNA activities. Chromosome Res 21, 627-641.

Kiani, J., Grandjean, V., Liebers, R., Tuorto, F., Ghanbarian, H., Lyko, F., Cuzin, F., and Rassoulzadegan, M. (2013). RNA-mediated epigenetic heredity requires the cytosine methyltransferase Dnmt2. PLoS Genet 9, e1003498.

Kim, I.S., Lee, M., Park, K.C., Jeon, Y., Park, J.H., Hwang, E.J., Jeon, T.I., Ko, S., Lee, H., Baek, S.H., et al. (2012). Roles of Mis18alpha in epigenetic regulation of centromeric chromatin and CENP-A loading. Mol Cell 46, 260-273.

Lacoste, N., Woolfe, A., Tachiwana, H., Garea, A.V., Barth, T., Cantaloube, S., Kurumizaka, H., Imhof, A., and Almouzni, G. (2014). Mislocalization of the Centromeric Histone Variant CenH3/CENP-A in Human Cells Depends on the Chaperone DAXX. Mol Cell 53, 631-644.

Lagana, A., Dorn, J.F., De Rop, V., Ladouceur, A.M., Maddox, A.S., and Maddox, P.S. (2010). A small GTPase molecular switch regulates epigenetic centromere maintenance by stabilizing newly incorporated CENP-A. Nat Cell Biol 12, 1186-1193.

Lermontova, I., Kuhlmann, M., Friedel, S., Rutten, T., Heckmann, S., Sandmann, M., Demidov, D., Schubert, V., and Schubert, I. (2013). Arabidopsis kinetochore null2 is an upstream component for centromeric histone $\mathrm{H} 3$ variant cenH3 deposition at centromeres. Plant Cell 25, 3389-3404.

Lermontova, I., Schubert, V., Fuchs, J., Klatte, S., Macas, J., and Schubert, I. (2006). Loading of Arabidopsis centromeric histone CENH3 occurs mainly during G2 and requires the presence of the histone fold domain. Plant Cell 18, 2443-2451. 
Liu, S.T., Rattner, J.B., Jablonski, S.A., and Yen, T.J. (2006). Mapping the assembly pathways that specify formation of the trilaminar kinetochore plates in human cells. $J$ Cell Biol 175, 41-53.

Lleres, D., James, J., Swift, S., Norman, D.G., and Lamond, A.I. (2009). Quantitative analysis of chromatin compaction in living cells using FLIM-FRET. J Cell Biol 187, 481496.

Loyola, A., and Almouzni, G. (2004). Histone chaperones, a supporting role in the limelight. Biochim Biophys Acta 1677, 3-11.

Luger, K., Mader, A.W., Richmond, R.K., Sargent, D.F., and Richmond, T.J. (1997). Crystal structure of the nucleosome core particle at 2.8 A resolution. Nature 389, 251260.

Luger, K., Rechsteiner, T.J., and Richmond, T.J. (1999). Preparation of nucleosome core particle from recombinant histones. Methods Enzymol 304, 3-19.

Luijsterburg, M.S., Lindh, M., Acs, K., Vrouwe, M.G., Pines, A., van Attikum, H., Mullenders, L.H., and Dantuma, N.P. (2012). DDB2 promotes chromatin decondensation at UV-induced DNA damage. J Cell Biol 197, 267-281.

Lusser, A., and Kadonaga, J.T. (2004). Strategies for the reconstitution of chromatin. Nat Methods 1, 19-26.

Maddox, P.S., Hyndman, F., Monen, J., Oegema, K., and Desai, A. (2007). Functional genomics identifies a Myb domain-containing protein family required for assembly of CENP-A chromatin. J Cell Biol 176, 757-763.

Maison, C., Bailly, D., Peters, A.H., Quivy, J.P., Roche, D., Taddei, A., Lachner, M., Jenuwein, T., and Almouzni, G. (2002). Higher-order structure in pericentric heterochromatin involves a distinct pattern of histone modification and an RNA component. Nat Genet 30, 329-334.

Mao, Y.S., Zhang, B., and Spector, D.L. (2011). Biogenesis and function of nuclear bodies. Trends Genet 27, 295-306. 
Marshall, O.J., Marshall, A.T., and Choo, K.H. (2008). Three-dimensional localization of CENP-A suggests a complex higher order structure of centromeric chromatin. J Cell Biol 183, 1193-1202.

Martin, R.M., and Cardoso, M.C. (2010). Chromatin condensation modulates access and binding of nuclear proteins. FASEB J 24, 1066-1072.

Matera, A.G., Terns, R.M., and Terns, M.P. (2007). Non-coding RNAs: lessons from the small nuclear and small nucleolar RNAs. Nat Rev Mol Cell Biol 8, 209-220.

Matson, D.R., Demirel, P.B., Stukenberg, P.T., and Burke, D.J. (2012). A conserved role for COMA/CENP-H/I/N kinetochore proteins in the spindle checkpoint. Genes Dev 26, $542-547$.

Mellone, B.G., Grive, K.J., Shteyn, V., Bowers, S.R., Oderberg, I., and Karpen, G.H. (2011). Assembly of Drosophila centromeric chromatin proteins during mitosis. PLoS Genet 7, e1002068.

Mendiburo, M.J., Padeken, J., Fulop, S., Schepers, A., and Heun, P. (2011). Drosophila CENH3 is sufficient for centromere formation. Science 334, 686-690.

Miell, M.D., Fuller, C.J., Guse, A., Barysz, H.M., Downes, A., Owen-Hughes, T., Rappsilber, J., Straight, A.F., and Allshire, R.C. (2013). CENP-A confers a reduction in height on octameric nucleosomes. Nat Struct Mol Biol 20, 763-765.

Mizuguchi, G., Xiao, H., Wisniewski, J., Smith, M.M., and Wu, C. (2007). Nonhistone $\mathrm{Scm} 3$ and histones CenH3-H4 assemble the core of centromere-specific nucleosomes. Cell 129, 1153-1164.

Moggs, J.G., Grandi, P., Quivy, J.P., Jonsson, Z.O., Hubscher, U., Becker, P.B., and Almouzni, G. (2000). A CAF-1-PCNA-mediated chromatin assembly pathway triggered by sensing DNA damage. Mol Cell Biol 20, 1206-1218.

Moree, B., Meyer, C.B., Fuller, C.J., and Straight, A.F. (2011). CENP-C recruits M18BP1 to centromeres to promote CENP-A chromatin assembly. J Cell Biol 194, 855871. 
Moreno-Moreno, O., Medina-Giro, S., Torras-Llort, M., and Azorin, F. (2011). The F box protein partner of paired regulates stability of Drosophila centromeric histone H3, CenH3(CID). Curr Biol 21, 1488-1493.

Moreno-Moreno, O., Torras-Llort, M., and Azorin, F. (2006). Proteolysis restricts localization of CID, the centromere-specific histone H3 variant of Drosophila, to centromeres. Nucleic Acids Res 34, 6247-6255.

Morey, L., Barnes, K., Chen, Y., Fitzgerald-Hayes, M., and Baker, R.E. (2004). The histone fold domain of Cse4 is sufficient for CEN targeting and propagation of active centromeres in budding yeast. Eukaryot Cell 3, 1533-1543.

Moroi, Y., Peebles, C., Fritzler, M.J., Steigerwald, J., and Tan, E.M. (1980). Autoantibody to centromere (kinetochore) in scleroderma sera. Proc Natl Acad Sci U S A 77, 1627-1631.

Muchardt, C., Guilleme, M., Seeler, J.S., Trouche, D., Dejean, A., and Yaniv, M. (2002). Coordinated methyl and RNA binding is required for heterochromatin localization of mammalian HP1alpha. EMBO Rep 3, 975-981.

Musacchio, A., and Salmon, E.D. (2007). The spindle-assembly checkpoint in space and time. Nat Rev Mol Cell Biol 8, 379-393.

Nakano, M., Cardinale, S., Noskov, V.N., Gassmann, R., Vagnarelli, P., Kandels-Lewis, S., Larionov, V., Earnshaw, W.C., and Masumoto, H. (2008). Inactivation of a human kinetochore by specific targeting of chromatin modifiers. Dev Cell 14, 507-522.

Nechemia-Arbely, Y., Fachinetti, D., and Cleveland, D.W. (2012). Replicating centromeric chromatin: spatial and temporal control of CENP-A assembly. Exp Cell Res $318,1353-1360$.

Nishino, T., Rago, F., Hori, T., Tomii, K., Cheeseman, I.M., and Fukagawa, T. (2013). CENP-T provides a structural platform for outer kinetochore assembly. EMBO J 32, 424436.

Nishino, T., Takeuchi, K., Gascoigne, K.E., Suzuki, A., Hori, T., Oyama, T., Morikawa, K., Cheeseman, I.M., and Fukagawa, T. (2012). CENP-T-W-S-X forms a unique centromeric chromatin structure with a histone-like fold. Cell 148, 487-501. 
Obuse, C., Yang, H., Nozaki, N., Goto, S., Okazaki, T., and Yoda, K. (2004). Proteomics analysis of the centromere complex from HeLa interphase cells: UV-damaged DNA binding protein 1 (DDB-1) is a component of the CEN-complex, while BMI-1 is transiently co-localized with the centromeric region in interphase. Genes Cells 9, 105120.

Ogiyama, Y., Ohno, Y., Kubota, Y., and Ishii, K. (2013). Epigenetically induced paucity of histone H2A.Z stabilizes fission-yeast ectopic centromeres. Nat Struct Mol Biol 20, 1397-1406.

Ohkuni, K., and Kitagawa, K. (2011). Endogenous transcription at the centromere facilitates centromere activity in budding yeast. Curr Biol 21, 1695-1703.

Okada, M., Cheeseman, I.M., Hori, T., Okawa, K., McLeod, I.X., Yates, J.R., 3rd, Desai, A., and Fukagawa, T. (2006). The CENP-H-I complex is required for the efficient incorporation of newly synthesized CENP-A into centromeres. Nat Cell Biol 8, 446-457.

Okada, M., Okawa, K., Isobe, T., and Fukagawa, T. (2009). CENP-H-containing complex facilitates centromere deposition of CENP-A in cooperation with FACT and CHD1. Mol Biol Cell 20, 3986-3995.

Okuwaki, M., Matsumoto, K., Tsujimoto, M., and Nagata, K. (2001). Function of nucleophosmin/B23, a nucleolar acidic protein, as a histone chaperone. FEBS Lett 506, 272-276.

Okuwaki, M., Tsujimoto, M., and Nagata, K. (2002). The RNA binding activity of a ribosome biogenesis factor, nucleophosmin/B23, is modulated by phosphorylation with a cell cycle-dependent kinase and by association with its subtype. Mol Biol Cell 13, 20162030.

Olszak, A.M., van Essen, D., Pereira, A.J., Diehl, S., Manke, T., Maiato, H., Saccani, S., and Heun, P. (2011). Heterochromatin boundaries are hotspots for de novo kinetochore formation. Nat Cell Biol 13, 799-808.

Ono, T., Fang, Y., Spector, D.L., and Hirano, T. (2004). Spatial and temporal regulation of Condensins I and II in mitotic chromosome assembly in human cells. Mol Biol Cell 15, 3296-3308. 
Ono, T., Losada, A., Hirano, M., Myers, M.P., Neuwald, A.F., and Hirano, T. (2003). Differential contributions of condensin I and condensin II to mitotic chromosome architecture in vertebrate cells. Cell 115, 109-121.

Ono, T., Yamashita, D., and Hirano, T. (2013). Condensin II initiates sister chromatid resolution during S phase. J Cell Biol 200, 429-441.

Panchenko, T., Sorensen, T.C., Woodcock, C.L., Kan, Z.Y., Wood, S., Resch, M.G., Luger, K., Englander, S.W., Hansen, J.C., and Black, B.E. (2011). Replacement of histone $\mathrm{H} 3$ with CENP-A directs global nucleosome array condensation and loosening of nucleosome superhelical termini. Proc Natl Acad Sci U S A 108, 16588-16593.

Park, Y.J., and Luger, K. (2006). Structure and function of nucleosome assembly proteins. Biochem Cell Biol 84, 549-558.

Pearson, C.G., Yeh, E., Gardner, M., Odde, D., Salmon, E.D., and Bloom, K. (2004). Stable kinetochore-microtubule attachment constrains centromere positioning in metaphase. Curr Biol 14, 1962-1967.

Perpelescu, M., and Fukagawa, T. (2011). The ABCs of CENPs. Chromosoma 120, 425446.

Perpelescu, M., Nozaki, N., Obuse, C., Yang, H., and Yoda, K. (2009). Active establishment of centromeric CENP-A chromatin by RSF complex. J Cell Biol 185, 397407.

Peterson, K.R. (2007). Preparation of intact yeast artificial chromosome DNA for transgenesis of mice. Nat Protoc 2, 3009-3015.

Pidoux, A.L., Choi, E.S., Abbott, J.K., Liu, X., Kagansky, A., Castillo, A.G., Hamilton, G.L., Richardson, W., Rappsilber, J., He, X., et al. (2009). Fission yeast Scm3: A CENPA receptor required for integrity of subkinetochore chromatin. Mol Cell 33, 299-311.

Podhraski, V., Campo-Fernandez, B., Worle, H., Piatti, P., Niederegger, H., Bock, G., Fyodorov, D.V., and Lusser, A. (2010). CenH3/CID incorporation is not dependent on the chromatin assembly factor CHD1 in Drosophila. PLoS One 5, e10120. 
Prendergast, L., van Vuuren, C., Kaczmarczyk, A., Doering, V., Hellwig, D., Quinn, N., Hoischen, C., Diekmann, S., and Sullivan, K.F. (2011). Premitotic assembly of human CENPs - T and $-\mathrm{W}$ switches centromeric chromatin to a mitotic state. PLoS Biol 9, e1001082.

Przewloka, M.R., Venkei, Z., Bolanos-Garcia, V.M., Debski, J., Dadlez, M., and Glover, D.M. (2011). CENP-C is a structural platform for kinetochore assembly. Curr Biol 21, $399-405$.

Rafalska-Metcalf, I.U., Powers, S.L., Joo, L.M., LeRoy, G., and Janicki, S.M. (2010). Single cell analysis of transcriptional activation dynamics. PLoS One 5, e10272.

Ranjitkar, P., Press, M.O., Yi, X., Baker, R., MacCoss, M.J., and Biggins, S. (2010). An E3 ubiquitin ligase prevents ectopic localization of the centromeric histone $\mathrm{H} 3$ variant via the centromere targeting domain. Mol Cell 40, 455-464.

Ransom, M., Dennehey, B.K., and Tyler, J.K. (2010). Chaperoning histones during DNA replication and repair. Cell 140, 183-195.

Regnier, V., Vagnarelli, P., Fukagawa, T., Zerjal, T., Burns, E., Trouche, D., Earnshaw, W., and Brown, W. (2005). CENP-A is required for accurate chromosome segregation and sustained kinetochore association of BubR1. Mol Cell Biol 25, 3967-3981.

Regulski, M., Lu, Z., Kendall, J., Donoghue, M.T., Reinders, J., Llaca, V., Deschamps, S., Smith, A., Levy, D., McCombie, W.R., et al. (2013). The maize methylome influences mRNA splice sites and reveals widespread paramutation-like switches guided by small RNA. Genome Res 23, 1651-1662.

Ribeiro, S.A., Vagnarelli, P., Dong, Y., Hori, T., McEwen, B.F., Fukagawa, T., Flors, C., and Earnshaw, W.C. (2010). A super-resolution map of the vertebrate kinetochore. Proc Natl Acad Sci U S A 107, 10484-10489.

Rice, J.C., Briggs, S.D., Ueberheide, B., Barber, C.M., Shabanowitz, J., Hunt, D.F., Shinkai, Y., and Allis, C.D. (2003). Histone methyltransferases direct different degrees of methylation to define distinct chromatin domains. Mol Cell 12, 1591-1598.

Saffery, R., Irvine, D.V., Griffiths, B., Kalitsis, P., Wordeman, L., and Choo, K.H. (2000). Human centromeres and neocentromeres show identical distribution patterns of $>20$ functionally important kinetochore-associated proteins. Hum Mol Genet 9, 175-185. 
Samoshkin, A., Arnaoutov, A., Jansen, L.E., Ouspenski, I., Dye, L., Karpova, T., McNally, J., Dasso, M., Cleveland, D.W., and Strunnikov, A. (2009). Human condensin function is essential for centromeric chromatin assembly and proper sister kinetochore orientation. PLoS One 4, e6831.

Sanchez-Pulido, L., Pidoux, A.L., Ponting, C.P., and Allshire, R.C. (2009). Common ancestry of the CENP-A chaperones Scm3 and HJURP. Cell 137, 1173-1174.

Savvidou, E., Cobbe, N., Steffensen, S., Cotterill, S., and Heck, M.M. (2005). Drosophila CAP-D2 is required for condensin complex stability and resolution of sister chromatids. J Cell Sci 118, 2529-2543.

Schleiffer, A., Kaitna, S., Maurer-Stroh, S., Glotzer, M., Nasmyth, K., and Eisenhaber, F. (2003). Kleisins: a superfamily of bacterial and eukaryotic SMC protein partners. Mol Cell 11, 571-575.

Schueler, M.G., and Sullivan, B.A. (2006). Structural and functional dynamics of human centromeric chromatin. Annu Rev Genomics Hum Genet 7, 301-313.

Schuh, M., Lehner, C.F., and Heidmann, S. (2007). Incorporation of Drosophila CID/CENP-A and CENP-C into centromeres during early embryonic anaphase. Curr Biol $17,237-243$.

Scott, M.S., Boisvert, F.M., McDowall, M.D., Lamond, A.I., and Barton, G.J. (2010). Characterization and prediction of protein nucleolar localization sequences. Nucleic Acids Res 38, 7388-7399.

Scott, M.S., Troshin, P.V., and Barton, G.J. (2011). NoD: a Nucleolar localization sequence detector for eukaryotic and viral proteins. BMC Bioinformatics 12, 317.

Screpanti, E., De Antoni, A., Alushin, G.M., Petrovic, A., Melis, T., Nogales, E., and Musacchio, A. (2011). Direct binding of Cenp-C to the Mis12 complex joins the inner and outer kinetochore. Curr Biol 21, 391-398.

Sekulic, N., Bassett, E.A., Rogers, D.J., and Black, B.E. (2010). The structure of (CENPA-H4)(2) reveals physical features that mark centromeres. Nature 467, 347-351. 
Shaiu, W.L., and Hsieh, T.S. (1998). Targeting to transcriptionally active loci by the hydrophilic N-terminal domain of Drosophila DNA topoisomerase I. Mol Cell Biol 18, 4358-4367.

Shang, W.H., Hori, T., Toyoda, A., Kato, J., Popendorf, K., Sakakibara, Y., Fujiyama, A., and Fukagawa, T. (2010). Chickens possess centromeres with both extended tandem repeats and short non-tandem-repetitive sequences. Genome Res 20, 1219-1228.

Shelby, R.D., Monier, K., and Sullivan, K.F. (2000). Chromatin assembly at kinetochores is uncoupled from DNA replication. J Cell Biol 151, 1113-1118.

Shelby, R.D., Vafa, O., and Sullivan, K.F. (1997). Assembly of CENP-A into centromeric chromatin requires a cooperative array of nucleosomal DNA contact sites. J Cell Biol 136, 501-513.

Shibahara, K., and Stillman, B. (1999). Replication-dependent marking of DNA by PCNA facilitates CAF-1-coupled inheritance of chromatin. Cell 96, 575-585.

Shintomi, K., and Hirano, T. (2011). The relative ratio of condensin I to II determines chromosome shapes. Genes Dev 25, 1464-1469.

Shirayama, M., Seth, M., Lee, H.C., Gu, W., Ishidate, T., Conte, D., Jr., and Mello, C.C. (2012). piRNAs initiate an epigenetic memory of nonself RNA in the C. elegans germline. Cell 150, 65-77.

Shivaraju, M., Camahort, R., Mattingly, M., and Gerton, J.L. (2011). Scm3 is a centromeric nucleosome assembly factor. J Biol Chem 286, 12016-12023.

Shivaraju, M., Unruh, J.R., Slaughter, B.D., Mattingly, M., Berman, J., and Gerton, J.L. (2012). Cell-cycle-coupled structural oscillation of centromeric nucleosomes in yeast. Cell 150, 304-316.

Shuaib, M., Ouararhni, K., Dimitrov, S., and Hamiche, A. (2010). HJURP binds CENP-A via a highly conserved $\mathrm{N}$-terminal domain and mediates its deposition at centromeres. Proc Natl Acad Sci U S A 107, 1349-1354. 
Silva, M.C., Bodor, D.L., Stellfox, M.E., Martins, N.M., Hochegger, H., Foltz, D.R., and Jansen, L.E. (2012). Cdk activity couples epigenetic centromere inheritance to cell cycle progression. Dev Cell 22, 52-63.

Soutoglou, E., Dorn, J.F., Sengupta, K., Jasin, M., Nussenzweig, A., Ried, T., Danuser, G., and Misteli, T. (2007). Positional stability of single double-strand breaks in mammalian cells. Nat Cell Biol 9, 675-682.

Stear, J.H., and Roth, M.B. (2002). Characterization of HCP-6, a C. elegans protein required to prevent chromosome twisting and merotelic attachment. Genes Dev 16, 14981508.

Stellfox, M.E., Bailey, A.O., and Foltz, D.R. (2013). Putting CENP-A in its place. Cell Mol Life Sci 70, 387-406.

Stoler, S., Rogers, K., Weitze, S., Morey, L., Fitzgerald-Hayes, M., and Baker, R.E. (2007). Scm3, an essential Saccharomyces cerevisiae centromere protein required for G2/M progression and Cse4 localization. Proc Natl Acad Sci U S A 104, 10571-10576.

Sullivan, B.A., and Karpen, G.H. (2004). Centromeric chromatin exhibits a histone modification pattern that is distinct from both euchromatin and heterochromatin. Nat Struct Mol Biol 11, 1076-1083.

Suzuki, A., Hori, T., Nishino, T., Usukura, J., Miyagi, A., Morikawa, K., and Fukagawa, T. (2011). Spindle microtubules generate tension-dependent changes in the distribution of inner kinetochore proteins. J Cell Biol 193, 125-140.

Tachiwana, H., Kagawa, W., Shiga, T., Osakabe, A., Miya, Y., Saito, K., HayashiTakanaka, Y., Oda, T., Sato, M., Park, S.Y., et al. (2011). Crystal structure of the human centromeric nucleosome containing CENP-A. Nature 476, 232-235.

Tagami, H., Ray-Gallet, D., Almouzni, G., and Nakatani, Y. (2004). Histone H3.1 and H3.3 complexes mediate nucleosome assembly pathways dependent or independent of DNA synthesis. Cell 116, 51-61.

Takayama, Y., Sato, H., Saitoh, S., Ogiyama, Y., Masuda, F., and Takahashi, K. (2008). Biphasic incorporation of centromeric histone CENP-A in fission yeast. Mol Biol Cell 19, 682-690. 
Takeuchi, K., Nishino, T., Mayanagi, K., Horikoshi, N., Osakabe, A., Tachiwana, H., Hori, T., Kurumizaka, H., and Fukagawa, T. (2014). The centromeric nucleosome-like CENP-T-W-S-X complex induces positive supercoils into DNA. Nucleic Acids Res 42, 1644-1655.

Teo, C.H., Lermontova, I., Houben, A., Mette, M.F., and Schubert, I. (2013). De novo generation of plant centromeres at tandem repeats. Chromosoma 122, 233-241.

Tomonaga, T., Matsushita, K., Yamaguchi, S., Oohashi, T., Shimada, H., Ochiai, T., Yoda, K., and Nomura, F. (2003). Overexpression and mistargeting of centromere protein-A in human primary colorectal cancer. Cancer Res 63, 3511-3516.

Topp, C.N., Zhong, C.X., and Dawe, R.K. (2004). Centromere-encoded RNAs are integral components of the maize kinetochore. Proc Natl Acad Sci U S A 101, 1598615991.

Tumbar, T., Sudlow, G., and Belmont, A.S. (1999). Large-scale chromatin unfolding and remodeling induced by VP16 acidic activation domain. J Cell Biol 145, 1341-1354.

Unhavaithaya, Y., and Orr-Weaver, T.L. (2013). Centromere proteins CENP-C and CAL1 functionally interact in meiosis for centromere clustering, pairing, and chromosome segregation. Proc Natl Acad Sci U S A 110, 19878-19883.

Vagnarelli, P. (2012). Mitotic chromosome condensation in vertebrates. Exp Cell Res $318,1435-1441$.

Vagnarelli, P., Hudson, D.F., Ribeiro, S.A., Trinkle-Mulcahy, L., Spence, J.M., Lai, F., Farr, C.J., Lamond, A.I., and Earnshaw, W.C. (2006). Condensin and Repo-Man-PP1 cooperate in the regulation of chromosome architecture during mitosis. Nat Cell Biol 8 , 1133-1142.

Valente, L.P., Silva, M.C., and Jansen, L.E. (2012). Temporal control of epigenetic centromere specification. Chromosome Res 20, 481-492.

Van Hooser, A.A., Ouspenski, II, Gregson, H.C., Starr, D.A., Yen, T.J., Goldberg, M.L., Yokomori, K., Earnshaw, W.C., Sullivan, K.F., and Brinkley, B.R. (2001). Specification of kinetochore-forming chromatin by the histone H3 variant CENP-A. J Cell Sci 114, 3529-3542. 
Volpe, T.A., Kidner, C., Hall, I.M., Teng, G., Grewal, S.I., and Martienssen, R.A. (2002). Regulation of heterochromatic silencing and histone H3 lysine-9 methylation by RNAi. Science 297, 1833-1837.

Wade, C.M., Giulotto, E., Sigurdsson, S., Zoli, M., Gnerre, S., Imsland, F., Lear, T.L., Adelson, D.L., Bailey, E., Bellone, R.R., et al. (2009). Genome sequence, comparative analysis, and population genetics of the domestic horse. Science 326, 865-867.

Wan, X., O'Quinn, R.P., Pierce, H.L., Joglekar, A.P., Gall, W.E., DeLuca, J.G., Carroll, C.W., Liu, S.T., Yen, T.J., McEwen, B.F., et al. (2009). Protein architecture of the human kinetochore microtubule attachment site. Cell 137, 672-684.

Wang, J., Liu, X., Dou, Z., Chen, L., Jiang, H., Fu, C., Fu, G., Liu, D., Zhang, J., Zhu, T., et al. (2014). Mitotic regulator Mis18beta interacts with and specifies the centromeric assembly of molecular chaperone HJURP. J Biol Chem.

Wilkins, B.J., Rall, N.A., Ostwal, Y., Kruitwagen, T., Hiragami-Hamada, K., Winkler, M., Barral, Y., Fischle, W., and Neumann, H. (2014). A cascade of histone modifications induces chromatin condensation in mitosis. Science 343, 77-80.

Williams, B.C., Murphy, T.D., Goldberg, M.L., and Karpen, G.H. (1998). Neocentromere activity of structurally acentric mini-chromosomes in Drosophila. Nat Genet 18, 30-37.

Williams, J.S., Hayashi, T., Yanagida, M., and Russell, P. (2009). Fission yeast Scm3 mediates stable assembly of Cnp1/CENP-A into centromeric chromatin. Mol Cell 33, 287-298.

Wong, L.H., Brettingham-Moore, K.H., Chan, L., Quach, J.M., Anderson, M.A., Northrop, E.L., Hannan, R., Saffery, R., Shaw, M.L., Williams, E., et al. (2007). Centromere RNA is a key component for the assembly of nucleoproteins at the nucleolus and centromere. Genome Res 17, 1146-1160.

Wong, L.H., McGhie, J.D., Sim, M., Anderson, M.A., Ahn, S., Hannan, R.D., George, A.J., Morgan, K.A., Mann, J.R., and Choo, K.H. (2010). ATRX interacts with H3.3 in maintaining telomere structural integrity in pluripotent embryonic stem cells. Genome Res 20,351-360. 
Xiao, H., Mizuguchi, G., Wisniewski, J., Huang, Y., Wei, D., and Wu, C. (2011). Nonhistone Scm3 binds to AT-rich DNA to organize atypical centromeric nucleosome of budding yeast. Mol Cell 43, 369-380.

Yoda, K., Ando, S., Morishita, S., Houmura, K., Hashimoto, K., Takeyasu, K., and Okazaki, T. (2000). Human centromere protein A (CENP-A) can replace histone H3 in nucleosome reconstitution in vitro. Proc Natl Acad Sci U S A 97, 7266-7271.

Yong-Gonzalez, V., Wang, B.D., Butylin, P., Ouspenski, I., and Strunnikov, A. (2007). Condensin function at centromere chromatin facilitates proper kinetochore tension and ensures correct mitotic segregation of sister chromatids. Genes Cells 12, 1075-1090.

Zasadzinska, E., Barnhart-Dailey, M.C., Kuich, P.H., and Foltz, D.R. (2013). Dimerization of the CENP-A assembly factor HJURP is required for centromeric nucleosome deposition. EMBO J 32, 2113-2124.

Zeitlin, S.G., Shelby, R.D., and Sullivan, K.F. (2001). CENP-A is phosphorylated by Aurora B kinase and plays an unexpected role in completion of cytokinesis. J Cell Biol $155,1147-1157$.

Zhang, W., Colmenares, S.U., and Karpen, G.H. (2012). Assembly of Drosophila centromeric nucleosomes requires CID dimerization. Mol Cell 45, 263-269.

Zhou, Z., Feng, H., Zhou, B.R., Ghirlando, R., Hu, K., Zwolak, A., Miller Jenkins, L.M., Xiao, H., Tjandra, N., Wu, C., et al. (2011). Structural basis for recognition of centromere histone variant $\mathrm{CenH} 3$ by the chaperone Scm3. Nature 472, 234-237. 


\section{Plasmids}

\begin{tabular}{|c|c|c|c|}
\hline pMB \# & Name & pMB \# & Name \\
\hline 1 & mcherry-LacI-HJURP & 25 & $\begin{array}{l}\text { Destination c-term } \\
\text { NCAPH-LAP }\end{array}$ \\
\hline 2 & $\begin{array}{l}\text { mcherry-LacI- } \\
\text { Mis18alpha }\end{array}$ & 26 & $\begin{array}{l}\text { Destination c-term } \\
\text { CAPH2-LAP }\end{array}$ \\
\hline 3 & TetR-3xHA & \multirow[t]{2}{*}{27} & $\begin{array}{l}\text { NCAPH entry clone } \\
\text { (stuke lab) from }\end{array}$ \\
\hline 4 & Flag-TetR & & orfeome \\
\hline 5 & $\begin{array}{l}\text { mCherry-LacI-HJScm3- } \\
\text { Cons }\end{array}$ & 28 & $\begin{array}{l}\text { CAPH2 entry clone } \\
\text { (stuke lab) }\end{array}$ \\
\hline 6 & $\begin{array}{c}\text { entry clone GFP-Scm } 3 \text { - } \\
\text { H2B }\end{array}$ & 29 & $\begin{array}{l}\text { HJFL lentivirus GW } \\
\text { dest vector c-term GFP }\end{array}$ \\
\hline 7 & $\begin{array}{c}\mathrm{H} 2 \mathrm{~B} \\
\text { Destination vector GFP- } \\
\text { Scm3-H2B }\end{array}$ & 30 & $\begin{array}{l}\text { HJdeltaconserved } \\
\text { lentivirus GW dest }\end{array}$ \\
\hline 8 & $\begin{array}{c}\text { Entry clone- } \\
\text { HJURPdeltaConserved, } \\
\text { no stop }\end{array}$ & 31 & $\begin{array}{l}\text { vector c-term GFP } \\
\text { gfp-LacI destination } \\
\text { vector }\end{array}$ \\
\hline \multirow[b]{2}{*}{9} & \multirow{2}{*}{$\begin{array}{l}\text { Entry clone- } \\
\text { HJURPdeltaConserved, } \\
\text { stop }\end{array}$} & 32 & $\begin{array}{l}\text { destination mCherry- } \\
\text { LacI-HJdeltaCons }\end{array}$ \\
\hline & & \multirow{2}{*}{33} & destination mCherry- \\
\hline \multirow{2}{*}{10} & \multirow{2}{*}{$\begin{array}{c}\text { Destination Vector- } \\
\text { HJURPdeltaConserved- } \\
\text { LAP }\end{array}$} & & $(201-348)$ \\
\hline & & 34 & $\begin{array}{c}\text { destination GFP- } \\
\text { HJdeltaCons }\end{array}$ \\
\hline 11 & $\begin{array}{c}\text { Destination Vector- } \\
\text { HJURPdeltaConserved- } \\
\text { LAP, stop }\end{array}$ & 35 & $\begin{array}{c}\text { destination GFP- } \\
\text { HJCons alone (201-348) }\end{array}$ \\
\hline 12 & mCherry-LacI-CENP-A & 36 & CAPD3-gfp \\
\hline 13 & $\begin{array}{l}\text { HJURPScm3-Cons with } \\
\text { stop }\end{array}$ & 37 & $\begin{array}{l}\text { NCAPH entry clone } \\
\text { with stop codon }\end{array}$ \\
\hline 14 & $\begin{array}{l}\text { HJURPScm3-Cons no } \\
\text { stop }\end{array}$ & 38 & $\begin{array}{l}\text { NCAPH } 2 \text { entry clone } \\
\text { with stop codon }\end{array}$ \\
\hline \multirow{2}{*}{15} & \multirow{2}{*}{$\begin{array}{l}\text { Entry clone } \\
\text { HJURPCons-R1-R2 no } \\
\text { stop }\end{array}$} & 39 & $\begin{array}{l}\text { MBP entry clone with } \\
\text { stop codon }\end{array}$ \\
\hline & & 40 & $\begin{array}{l}\text { NCAPH destination } \\
\text { clone (mchlacI) }\end{array}$ \\
\hline 16 & $\begin{array}{l}\text { Entry clone } \\
\text { HJURPCons-R1-R2 } \\
\text { with stop }\end{array}$ & 41 & $\begin{array}{l}\text { NCAPH2 destination } \\
\text { clone (mchlacI) }\end{array}$ \\
\hline 17 & $\begin{array}{l}\text { MBP-HJURPScm3- } \\
\text { Conserved }\end{array}$ & 42 & $\begin{array}{l}\text { MBP destination clone } \\
\text { (mchlacI) }\end{array}$ \\
\hline 18 & $\begin{array}{c}\text { MBP- } \\
\text { HJURPConserved-R1- }\end{array}$ & 43 & $\begin{array}{c}\mathrm{HJ} \text { aa } 348-555 \text { no stop } \\
\text { entry clone }\end{array}$ \\
\hline & R2 & 44 & HJ aa $555-748$ no stop \\
\hline & mCherry-LacI- & 44 & entry clone \\
\hline 19 & $\begin{array}{c}\text { HJURPConserved-R1- } \\
\text { R2 }\end{array}$ & 45 & $\begin{array}{c}\mathrm{HJ} \text { aa } 348-748 \text { no stop } \\
\text { entry clone }\end{array}$ \\
\hline 20 & $\begin{array}{l}\text { hNpm1 with stop entry } \\
\text { clone }\end{array}$ & 46 & $\begin{array}{l}\text { HJ aa } 348-555 \text { no stop- } \\
\text { Cterm HA destination }\end{array}$ \\
\hline & hNpm1 no stop entry & & clone \\
\hline 21 & clones & & HJ aa 555-748 no stop- \\
\hline 22 & $\begin{array}{l}\text { GBP with stop entry } \\
\text { clone }\end{array}$ & 47 & $\begin{array}{l}\text { Cterm HA destination } \\
\text { clone }\end{array}$ \\
\hline 23 & GBP no stop entry clone & 48 & $\begin{array}{l}\text { HJ aa } 348-748 \text { no stop } \\
\text { Cterm HA destination }\end{array}$ \\
\hline 24 & mCherry-LacI-Npm 1 & & clone \\
\hline 24 & destination vector & & HJ aa $1-348$ no stop \\
\hline 25 & $\begin{array}{l}\text { Destination c-term } \\
\text { NCAPH-LAP }\end{array}$ & 49 & $\begin{array}{l}\text { Cterm HA destination } \\
\text { clone }\end{array}$ \\
\hline
\end{tabular}

\section{Plasmid Reference Table}




\section{$\underline{\text { Antibodies }}$}

\begin{tabular}{|c|c|c|c|c|}
\hline Antibody & $\begin{array}{l}\text { Product info if } \\
\text { applicable }\end{array}$ & Species & Dilution & Application \\
\hline CENP-A & Abcam 13939 & Mouse & $1: 1000$ & IF, WB \\
\hline CENP-C & Custom & Mouse & $1: 1000$ & IF \\
\hline CENP-T & 3408 & Rabbit & $1: 2000$ & IF, WB \\
\hline HJURP & 3399 & Rabbit & $1: 1000$ & WB, IF \\
\hline Mis18BP1 & Bethyl 10285 & Rabbit & $1: 1000$ & WB \\
\hline Npm1 & Custom & Mouse & $1: 8000$ & IF, WB \\
\hline LacI & Millipore 9A5 & Mouse & $1 \mu \mathrm{g} / \mathrm{mL}$ & WB \\
\hline GFP & 3404 & Rabbit & $\begin{array}{l}1: 1000 \mathrm{IF}, \mathrm{WB} \\
1 \mu \mathrm{g} / \mathrm{mL} \text { for IP }\end{array}$ & IF, WB, IP \\
\hline Pan Histone H3 & Abcam 1791-100 & Rabbit & $2 \mu \mathrm{g} / 10^{6}$ cells & IP \\
\hline $\mathrm{HA}$ & Covance HA.11 & Mouse & $1: 1000$ & IF, WB, IP \\
\hline MBP-HRP & NEB E80385 & Mouse & $1: 10,000$ & WB \\
\hline His & Santa Cruz sc-803 & Rabbit & $1: 500$ & WB \\
\hline $\mathrm{ACA}$ & $\begin{array}{l}\text { Antibodies } \\
\text { Incorporated } \\
15-235\end{array}$ & Human & $\begin{array}{c}1: 1000 \text { for } \mathrm{IF}, \\
1: 500 \mathrm{WB}\end{array}$ & IF, WB \\
\hline Hecl & $\begin{array}{c}\text { Genetex } \\
\text { GTX70268 }\end{array}$ & Mouse & $1: 1000$ & IF \\
\hline
\end{tabular}

Antibody Reference Table 


\section{siRNA}

\begin{tabular}{|c|c|c|c|}
\hline siRNA & Sense sequence 5 ' to $3^{\prime}$ & Product Info & Concentration for use \\
\hline HJURP cds & CAAGUAUGGAAGUUCGAUAtt & $\begin{array}{c}\text { Pre-designed Silencer Select } \\
\text { siRNA ID: s30814 } \\
\text { LifeTechnologies }\end{array}$ & $20 \mathrm{nM}$ \\
\hline HJURP 5' UTR & GGGUUGGCGCUUGGGUACUUU & $\begin{array}{l}\text { Custom Silencer Select } \\
\text { LifeTechnologies }\end{array}$ & 20 nM (use with 3') \\
\hline HJURP 3' UTR & GAGAUAACCUCGAGUUCUUUU & $\begin{array}{l}\text { Custom Silencer Select } \\
\text { LifeTechnologies }\end{array}$ & 20 nM (use with 5') \\
\hline CAP-H2 & UUCCAGAGAUGAAAUCAAGGGCCUG & $\begin{array}{l}\text { Custom Stealth siRNA } \\
\text { LifeTechnologies }\end{array}$ & $33.3 \mathrm{nM}$ \\
\hline CAP-D3 & AGGAAUUCAAGUUAACAGAGGCUUG & $\begin{array}{l}\text { Custom Stealth siRNA } \\
\text { LifeTechnologies }\end{array}$ & $50 \mathrm{nM}$ \\
\hline Mis18BP1 (C14orf106) & GGAUAUCCAAAUUAUCUCAtt & $\begin{array}{c}\text { Pre-designed Silencer Select } \\
\text { siRNA ID: s30721 } \\
\text { LifeTechnologies }\end{array}$ & $20 \mathrm{nM}$ \\
\hline Mis $18 \alpha$ & GAAGAUGUCUUGAAAGCAUTT & $\begin{array}{c}\text { Pre-designed Silencer Select } \\
\text { LifeTechnologies }\end{array}$ & $5 \mathrm{nM}$ \\
\hline
\end{tabular}

siRNA Reference Table 